\title{
Cortical patterning and neuronal migration are under the guide of BAF complex functionality
}

\author{
Dissertation \\ for the award of the degree \\ "Doctor rerum naturalium" \\ Faculty of Biology \\ of the Georg-August-University of Goettingen
}

within the doctoral program Molecular Physiology of the Brain of the Georg-August University School of Science (GAUSS)

Submitted by

Godwin Sokpor

Born in Accra, Ghana

Goettingen, 2019 


\section{Thesis Advisory Committee Members}

Prof. Dr. Jochen Staiger (1st)

Department of Neuroanatomy, University Medical Center Goettingen

Prof. Dr. Ivan Manzini (2nd)

Department of Animal Physiology and Molecular Biomedicine, University of Giessen

Dr. Kristine Henningfeld (3rd)

Department of Developmental Biochemistry, University Medical Center Goettingen

\section{Members of the Examination Board:}

Prof. Dr. Jochen Staiger (First Reviewer)

Department of Neuroanatomy, University Medical Center Goettingen

Prof. Dr. Ivan Manzini (Second Reviewer)

Department of Animal Physiology and Molecular Biomedicine, University of Giessen

\section{Further members of the Examination Board:}

Dr. Kristine Henningfeld

Department of Developmental Biochemistry, University Medical Center Goettingen

Prof. Dr. Thomas Dresbach

Department of Anatomy and Embryology, University Medical Center Goettingen

Prof. Dr. Ahmed Mansouri

Molecular Cell Differentiation Group, University of Goettingen

Prof. Dr. Anastassia Stoykova

Department of Genes and Behaviour, University of Goettingen

Date of the oral examination: November 27, 2019 


\section{Declaration}

I hereby declare that the $\mathrm{PhD}$ thesis entitled "Cortical patterning and neuronal migration are under the guide of BAF complex functionality" is a product of my intellect, except for sources and aids cited.

Godwin Sokpor

Goettingen, September 30, 2019 


\section{TABLE OF CONTENTS}

Chapter 1: General Introduction

Chapter 2: Epigenetic regulation by BAF ( $\mathrm{mSWI} / \mathrm{SNF}$ ) chromatin remodeling complexes is indispensable for embryonic development

Chapter 3: BAF (mSWI/SNF) complex regulates mediolateral patterning in developing forebrain

Chapter 4: Loss of BAF complex in developing cortex perturbs neuronal migration and process outgrowth in a WNT signaling-dependent manner

Chapter 5: General discussion

General summary

References

List of figures

List of abbreviations

Acknowledgements 


\section{CHAPTER 1: General introduction}

Some elements in this chapter have already been published in:

(i) Sokpor G*, Castro-Hernandez R*, Rosenbusch J, Staiger JF, Tuoc T. 2018. ATPDependent Chromatin Remodeling During Cortical Neurogenesis. Front Neurosci 12: 226.

(ii) Sokpor $\mathrm{G}^{*}$, Xie $\mathrm{Y}^{*}$, Rosenbusch J, Tuoc T. 2017. Chromatin Remodeling BAF (SWI/SNF) Complexes in Neural Development and Disorders. Front Mol Neurosci 10: 243.

${ }^{*}$ Authors with equal contribution.

Personal contributions: I wrote the introduction, the part on chromatin remodeling in cortical development, and synthesized the two major aspects of the review in (i). The part on the properties of the BAF complex and its neurodevelopmental functions in (ii) were written by me. I also contributed to synthesizing the neurodevelopment and brain disease aspect of (ii).

\subsection{Development of the mammalian brain}

The complexities in the anatomy and function of the mammalian brain is reflective of the many cell biological and molecular events governing its formation. Beginning from the stage of neural induction, the embryonic brain cells or tissues are believed to be instructed by a myriad of factors leading to their specification, expansion, transformation, organization, and specialization, in order to make the brain have its correct form to be able to perform the many high-level cognitive and sensorimotor functions. Although the developmental processes which drive brain organogenesis are interlinked, it is possible to isolate the contribution and effect of specific processes that 
participate in the morphogenesis of the brain. Notable among them are the very early patterning processes: required for the structural and functional division of the brain, neurogenesis and gliogenesis: which involve the generation of the brain parenchyma, and the process of migration: needed for proper placement of neural cells for optimal maturation. The integrity of the brain cannot accommodate compromise of the normal progression of such separable neurodevelopmental events because of their indispensable contributions to proper brain genesis and functionality.

\subsubsection{Early patterning of the dorsal telencephalon during mammalian forebrain development}

After neural induction, the most rostral/anterior aspect of the neural tube develops into the left and right telencephalic vesicles which become the future hemispheres of the cerebral cortex following robust topological changes. The telencephalic vesicles undergo a major subdivision into the dorsal (pallium) and ventral (subpallium) telencephalon under the instruction of the dorsal midline roof plate. Essentially, it is the dorsal part of the telencephalon that transforms into the cortex proper and dorsal midline when it is exposed to several morphogenetic signaling molecules secreted by specialized domains referred to as "organizers". The cortical organizers include the cortical hem, antihem, anterior neural ridge, and roof plate. Molecular products of these cortical organizing centers, together with the activity of other factors with diffusible or graded expression, direct regionalization and arealization of the cerebral cortex (Fig.

1.1; (Wilson \& Rubenstein, 2000; Rallu et al., 2002; O'Leary et al., 2007)). 
A

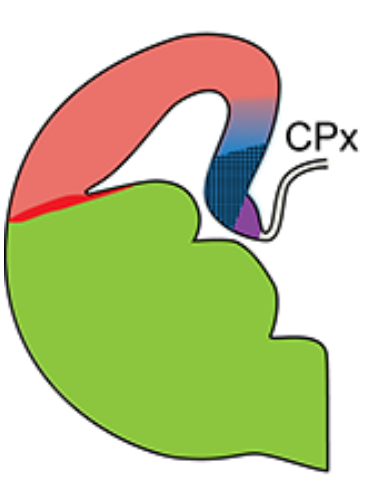

Cortical hem

四 Hippocampal primordium

nedial palium

neocortex

घAntihem

- Ventral telencephalon
B

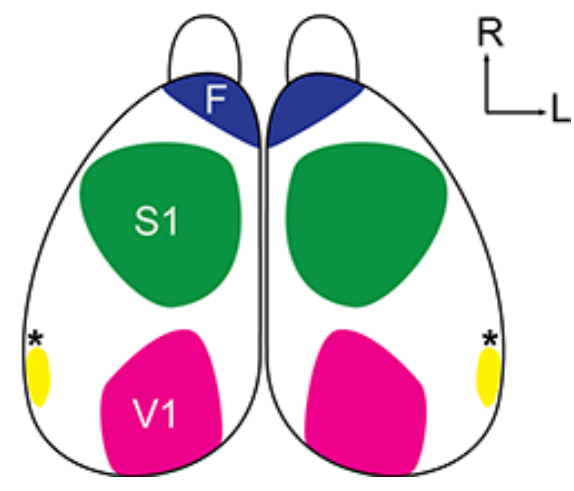

Figure 1.1 Schematics showing general patterning of the mouse telencephalon or cortex: $(A)$ Illustrates regional telencephalic division in which the dorsal part of the telencephalon is demarcated from the ventral aspect by the antihem (palial-subpallial border). The neocortex and medial cortex comprise the dorsal telencephalon. The medial cortex is in turn made up of the hippocampal anlage, cortical hem, and choroid plexus (CPx). (B) Shows illustration of the areal and/or functional sub-division of the cortex. The motor/frontal (F), primary sensory (S1), primary visual (V1) and primary auditory (A1, asterisked) cortical areas are indicated. Rostral (R) and lateral (L) orientations are shown.

The specification and regionalization of the dorsal telencephalon is regulated by some transcription factors, and secreted signaling molecules called morphogens (Fig. 1.2). Neurogenins 1 and 2, which belong to the basic helix-loop-helix (bHLH) family of transcription factors are expressed in dorsal telencephalic progenitors and reported to contribute to the establishment of dorsal telencephalic identity (Fode et al., 2000). The exclusion of the ventral telencephalic maker Mash1, a bHLH protein, from the dorsal telencephalon is necessary to allow cortical specification (Fode et al., 2000). The zinc finger transcription factor Gli3 and LIM homeobox transcription factor LHX2 are also powerful determinants of cortical regions. Lack of Gli3 resulted in perturbation of medial cortical region (hippocampal identity) in favor of the acquisition of ventral telencephalic identity (Tole et al., 2000). LHX2 on the other hand is a definitive selector of neocortical identity. It selects and specifies dorsal telencephalic progenitors to adopt a cortical ventricular zone (VZ) progenitor fate leading to formation of the neocortex (Monuki et al., 2001; Mangale et al., 2008). 


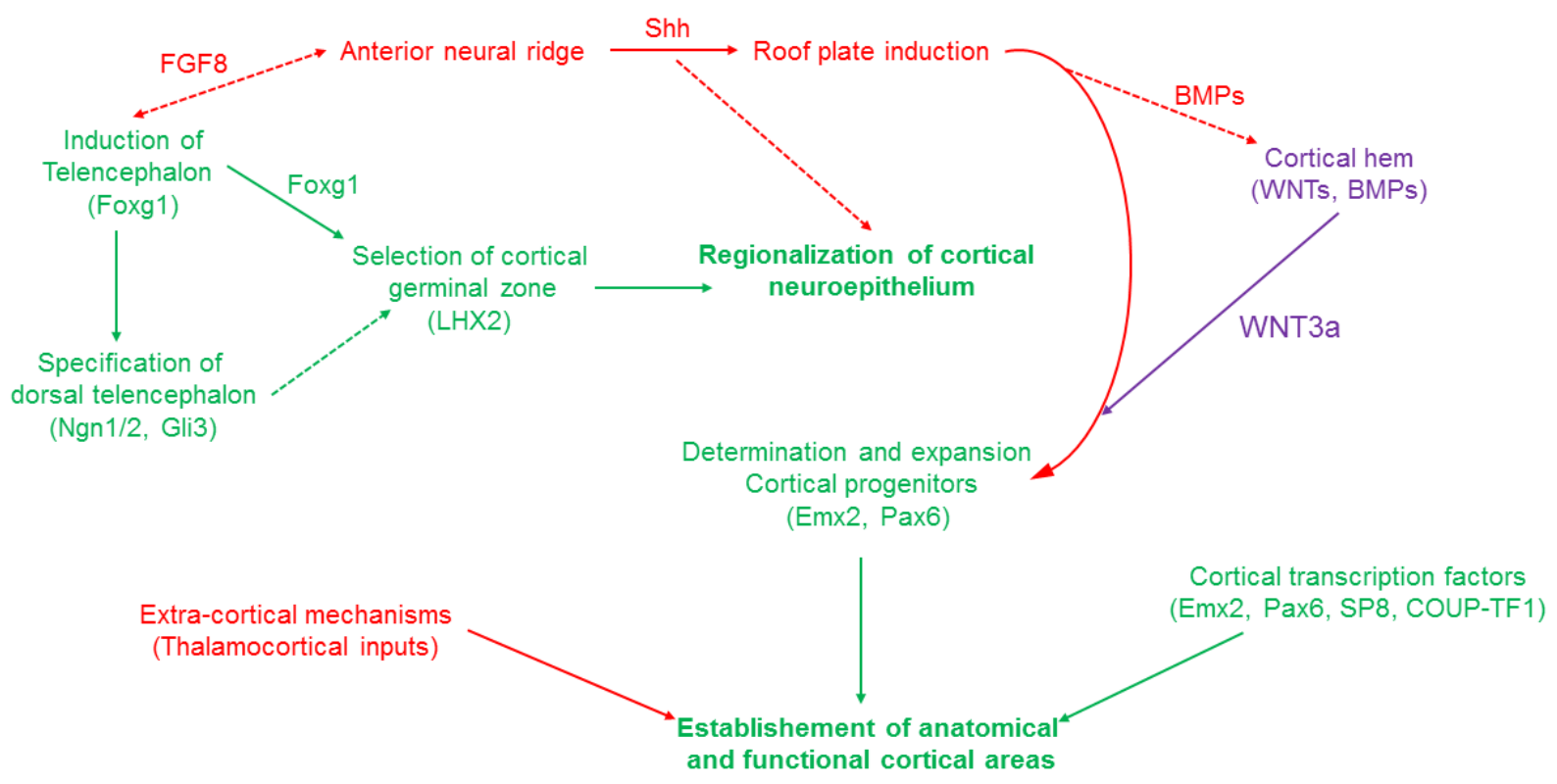

Figure 1.2 Synopsis of the regulatory events during cortical patterning: Induction of the telencephalon is initiated by the anterior neural ridge-FGF8-Foxg1 pathway coupled with activity of the dorsal midline roof plate which is in turn regulated by the sonic hedgehog (Shh) pathway. The dorsal aspect of the telencephalon is specified under the instruction of factors, including neurogenins (1 and 2 ) and Gli3. The transcription factor LHX2 then confers cortical ventricular zone fate to a portion of the dorsal telencephalic neural progenitors, with some hierarchical modulatory input from Foxg1 and probably from the neurogenins. The later event, alongside possible response to secretions from the anterior neural ridge lead to regional patterning of the cortical primordium. The transcription factors Emx2 and Pax6, and morphogens secreted directly or indirectly from the dorsal midline structures (roof plate, and cortical hem) are key determinants of cortical progenitor identity. Ultimately, the cortex is arealized by the graded expression of morphogens and some other transcription factors as indicated. Notably, extrinsic mechanism, including thalamocortical inputs, may contribute to the establishment of functional cortical areas. Arrows with broken line indicate possible effect. Red, green and purple labels refer to events outside, inside, and from the cortical hem, respectively. Modified from (Monuki \& Walsh, 2001).

The cortical hem, a dorsal midline structure bordered by the choroid plexus and hippocampal anlage, also plays critical role in the regionalization of the dorsal telencephalon (Fig. 1.1A). It is a hub for the secretion of morphogens needed for early cortical patterning. Key among such hem-derived morphogens are the WNT (Grove et al., 1998) and BMP (Furuta et al., 1997) proteins. The WNT signaling factors in particular are involved in priming the adjacent medial cortical VZ progenitors that results in the induction of the hippocampal primordium (Galceran et al., 2000; Lee et al., 2000; Zhou et al., 2004; Machon et al., 2007). 
On the other hand, arealization or areal patterning of the cortex is the process by which the neocortex is organized into discrete anatomical domains that subserve the sensorimotor functions of the mammalian cortex (Fig. 1.1B, (O'Leary et al., 2007)). Morphogens also play central roles in such areal patterning of the cortex. They have a local source of production from which they diffuse to other locations to establish their morphogenic effect(s)-patterning in this context (Borello \& Pierani, 2010). Morphogens from the patterning centers, cortical hem (WNTs and BMPs), and commissural plate (formerly the anterior neural ridge) (FGF8 and FGF17) are notable regulators of cortical arealization. Likewise, graded expression of transcription factors such as Pax6, Emx2, Sp8, and COUP-TF1 is necessary for the determination of cortical areas and sizes during cortical arealization (Fig. 1.1; (reviewed in (O'Leary et al., 2007)).

\subsubsection{Abnormal regulation of early dorsal telencephalic patterning can perturb cortical regionalization and arealization}

The mechanistic interplay between several regulatory factors of early patterning of the dorsal telencephalon is critical for correcting subdivision and later ontology of the cortex. Distortion of any aspect of the said regulatory network usually results in abnormal patterning. For instance, during early patterning, the interaction between morphogens belonging to the FGF, WNT, and BMP families play pivotal roles in the specification and regionalization of the dorsal telencephalon (Shimogori et al., 2004). Consequently, their dysregulation greatly disrupts proper formation of the dorsal telencephalon, including later cortical morphogenesis like formation of the hippocampus and corpus callosum (Grove et al., 1998; Theil et al., 1999; Tole et al., 2000; Crossley et al., 2001; Ohkubo et al., 2002; Shimogori et al., 2004; Storm et al., 
2006; Benadiba et al., 2012; Amaniti et al., 2013; Magnani et al., 2014). Interestingly, Gli3 has been identified as the factor that centrally regulates the interactions between the aforementioned morphogens and to maintain their homeostatic balance (Hasenpusch-Theil et al., 2017). Accordingly, ablation of Gli3 leads to overexpression of FGFs, whereas WNTs and BMPs are downregulated (Grove et al., 1998; Theil et al., 1999; Kuschel et al., 2003; Rash \& Grove, 2007).

Similarly, disturbance in the expression of other key transcription factors known to play significant roles in early cortical patterning can have implications for abnormal cortical patterning. Some of these transcription factors specifically act to regulate the formation and placement of the cortical hem which is a notable telencephalic organizer (Subramanian \& Tole, 2009a). The transcription factors FOXG1 and LHX2, for instance, interact hierarchically to regulate the size of the cortical hem and by extension the dorsal telencephalon (Godbole et al., 2018). A dorsal telencephalon with an exaggerated cortical hem size, reduced/lateralized neocortex, and without hippocampal primordia results when FOXG1 and LHX2 are mis-expressed in the early developing telencephalon (Bulchand et al., 2001; Monuki et al., 2001; Mangale et al., 2008; Roy et al., 2014; Godbole et al., 2018). Commonly, perturbation of many of the factors that regulate pattering of the dorsal telencephalon by acting on the hem, results in phenotypes including, fate change of hem progenitors, expansion or reduction of the hem size, reduced in hippocampal anlage or absolute absence of it leading to loss of medial cortical patterning, expansion or absence of the antihem, reduced or complete absence of the cortical primordium/neocortex, and expansion of the choroid plexus (Xuan, SH et al., 1995; Huh et al., 1999; Monuki et al., 2001; Muzio \& Mallamaci, 2005; Mangale et al., 2008; Chizhikov et al., 2010; Lavado et al., 2013; Caronia-Brown et al., 2014; Godbole et al., 2018). 
The formation of cortical areas are also very much tightly regulated by some transcription factors. The gradient expression of the transcription factors Pax6, Emx2, Sp8, and COUP-TF1 in cortical progenitors have been implicated in the regulation of cortical lamination and areal identity leading to the formation of the various functional cortical territories (Fig. 1.2). Such graded expression pattern is partly established by the involvement of morphogens produced by the cortical hem and commissural plate during cortical patterning (Bishop et al., 2000; Mallamaci et al., 2000; Viti et al., 2003; Lillien \& Gulacsi, 2006; Armentano et al., 2007; O'Leary et al., 2007; Zembrzycki et al., 2007; Toyoda et al., 2010). If the expression of the aforementioned transcription factors is disturbed in the developing mouse cortex, it leads to striking distortions in cortical areal patterning with potential implications for abnormal cortical function (O'Leary et al., 2007). When Pax6 gene is deleted in the early developing mouse cortical primordium, the resultant E18.5 cortex displays an expanded primary visual area (V1) leading to reduction and rostral shift of the other functional cortical areas (primary motor [M], sensory [S1] and auditory [A1] areas), therefore, departing from the normal arealized cortical patterns (Fig. 1.1B). This cortical areal phenotype is recapitulated in the Sp8-ablated late embryonic cortex (Zembrzycki et al., 2007). Dosage reduction in the amount of Emx2 expression (i.e. Emx2 heterozygosity) or upregulation of Emx2 expression in developing mouse cortex has been reported to also alter normal areal patterning of the cortex (Hamasaki et al., 2004). More markedly, the E18.5 Emx2 null cortex is abnormally arealized and presents with enlargement of $\mathrm{M}$ area, enlargement and posterior shift of $\mathrm{S} 1$, and dramatic reduction and posterior shift of $\mathrm{A} 1$ and $\mathrm{V} 1$ (Bishop et al., 2000; Mallamaci et al., 2000; Muzio et al., 2002; Muzio \& Mallamaci, 2003; Hamasaki et al., 2004; Li et al., 2006). Similarly, when COUP-TF1 expression is conditionally abolished in the developing cortex, the motor cortex enlarges but more 
drastically leading to marked size reduction and posterior shift of the other cortical areas (Armentano et al., 2007). The above mentioned cortical areal phenotypes are elegantly presented (in vivo and schematized) in O'Leary et al., (2007).

Given that patterning programs in cortical progenitors are transferred to their progenies (O'Leary \& Sahara, 2008), it is possible that such cues may regulate postmitotic neurodevelopmental events such as the generation of neurons and their areal placement via migration to achieve the cytoarchitectural organization of the various cortical regions or areas. This brings into focus how neurogenesis and neuronal migration contribute to the regional and/or areal morphogenesis of the cortex. An understanding of regulatory mechanisms involved will thus provide further insights into the essentialities of late patterning events during cortical formation.

\subsubsection{Neuronal generation and migration are late cortical patterning events crucial for cortical laminar formation}

After the cortical neuroepithelium has been properly specified or patterned, the neuroepithelial cells therein undergo molecular and structural transformation to become radial glial $(R G)$ cells starting from E10.5-E12.5 in mice (Mollgoard \& Saunders, 1975; Aaku-Saraste et al., 1996; Hartfuss et al., 2001; Noctor et al., 2002; Malatesta et al., 2003; Haubensak et al., 2004; Noctor et al., 2004; Gotz \& Huttner, 2005; Kriegstein \& Alvarez-Buylla, 2009; Sahara \& O'Leary, 2009). In principle, this progenitor cell transition marks the commencement of cortical neurogenesis which largely determines morphogenesis of the cortex (Gotz \& Huttner, 2005; Kriegstein \& Alvarez-Buylla, 2009; Martinez-Cerdeno \& Noctor, 2016). When nascent neurons are generated, they actively migrate from their place of birth to be placed in their respective cortical layers (Fig. 1.3). Cohorts of newly generated neurons maintain their parent 
progenitor cell expression profile of patterning morphogens or transcriptional factors during the course of migration and in the cortical plate. It implies that cortical patterning events likely continue to go on at the postmitotic cell level to afford finer cortical patterning, including correct lamination and arealization of the neocortex (O'Leary \& Sahara, 2008).

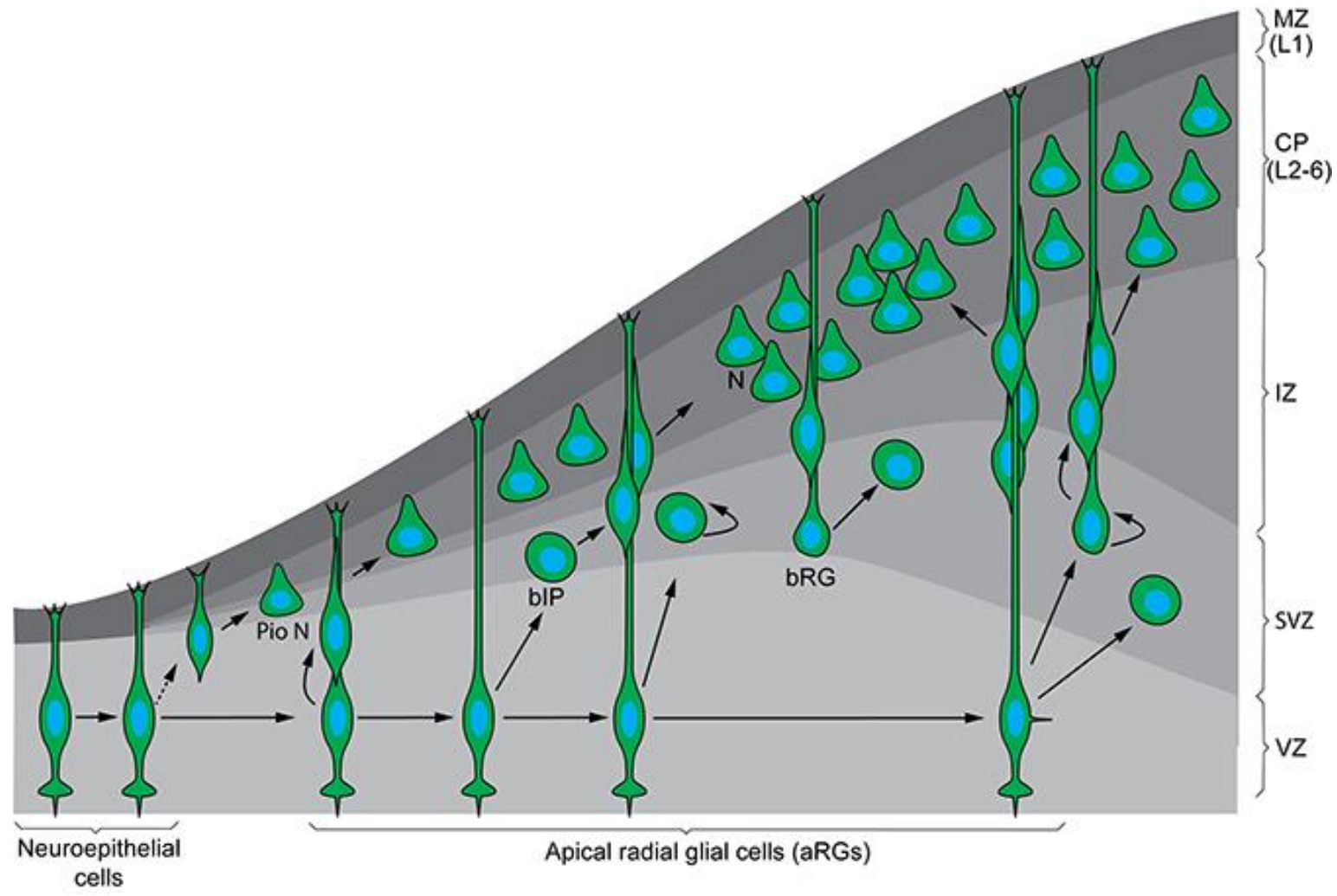

Figure 1.3 Illustration showing the generation and placement of excitatory neurons during cortical formation: Cortical neurogenesis commences soon after neuroepithelial cells (NEs) transform into apical radial glial cells (aRGs), around which time pioneer neurons (Pio Ns) are directly generated by NEs or RGs to establish nascent cortical regions. Later in embryonic cortical development, RGs produce neurons indirectly via neurogenic amplification division of basal intermediate progenitor (bIP) cells or basal radial glial (bRG) cells. Figure modified from (Sokpor et al., 2018).

\subsubsection{Cortical neurogenesis and layer formation}

The bulk of (excitatory) neurons that make up the cortex are generated in the germinal zones of the dorsal telencephalon during embryonic stages of mouse cortical development (Angevine \& Sidman, 1961). These neurons are thought to be generated by a diverse population of neuronal progenitors which can classified based on their 
morphologies, fate commitment, molecular features, and place of mitotic division (Lui et al., 2011; Florio \& Huttner, 2014; Taverna et al., 2014; Dehay et al., 2015; Fernandez et al., 2016). Generally, neural progenitors can be categorized into apical (AP) and basal progenitor (BP) cells. Neuroepithelial and radial glial cells (RGs) are examples of APs.

Many APs extend the full thickness of the developing cortical wall and their progenies are mainly either neurons or BPs. RGs in particular have apical and basal process anchorages with their soma residing in the VZ (Fig. 1.3; (Cameron \& Rakic, 1991; Bentivoglio \& Mazzarello, 1999; Gotz \& Huttner, 2005; Kriegstein \& Alvarez-Buylla, 2009)). Clonal lineage tracing revealed that RGs are the source of nearly all excitatory neurons in the developing cortex (Gotz \& Huttner, 2005; Kriegstein \& Alvarez-Buylla, 2009; Wilsch-Brauninger et al., 2016). Many BPs, on the other hand, are adventricularly located (subventricular/outer subventricular zone) and do not have such apical or basal anchoring processes except for a sub-population called basal outer RGs, some of which display basal connection (Haubensak et al., 2004; Miyata et al., 2004; Noctor et al., 2004; Sessa et al., 2008; Kowalczyk et al., 2009; Lui et al., 2011; Dehay et al., 2015). The BPs are known to amplify the neuronal output of RGs, and their population in the developing cortex is believed to increase with cortical evolution (Hansen et al., 2010; Kelava et al., 2012; Betizeau et al., 2013; Lewitus et al., 2013; Borrell \& Gotz, 2014; Romero \& Borrell, 2017). Generally, the generation of neurons from RGs (i.e. APs) is referred to as direct neurogenesis and contributes to the formation of deep cortical layers (Guillemot, 2005), whereas indirect neurogenesis is when neurons are produced from BPs such as intermediate progenitors that are mainly responsible for the formation of superficial cortical layers (Fig 1.3; (Sessa et al., 2008)) 
After progenies of neurogenic progenitors have received adequate spatiotemporal differentiation stimuli, they exist the cell cycle as postmitotic neurons. Pioneering postmitotic neurons born around E10.5 in the developing mouse cortex form the preplate which is subsequently split into the subplate and the marginal zone (MZ) by the first cohort of cortical projection neurons that express Tbr1 and born around E11.5; leading to the establishment of the first cortical layer (L6) in the cortical plate (CP). The mammalian cortical $M Z$ develops into $\mathrm{L} 1$ which is populated by tangentially migrated Reelin-expression Cajal-Retzius cells, whereas the subplate persists as noticeable strip of neurons just below L6 (Molliver et al., 1973; Marin-Padilla, 1978). Subsequent waves of temporally generated cortical neurons migrate to form the other cortical layers L5-2 (Molyneaux et al., 2007). With their differentiation program switched on, the newly generated neurons undergo extensive structural and functional transformation that permit their circuit integration in the cortex.

As already mentioned, patterning cues in neural progenitors are known to linger in their postmitotic neuronal progenies. Initially, the expression profile of such patterning cues are graded but as they differentiate further, various neuronal cohorts display distinct gene expression boarders that delineate them. Such patterning of gene expression at the postmitotic level together with other extrinsic factors such as thalamic cortical sensory inputs relayed from the periphery are believed to underscore proper structural and functional parcellation of the cortex (reviewed in (O'Leary \& Sahara, 2008)).

\subsubsection{Migration of principal cortical neurons during corticogenesis}

Following the temporal generation of neuronal subtypes from progenitors, the newly born neuron undergoes complex movement from the VZ or subventricular zone (SVZ) through the intermediate zone (IZ) to be placed in its specified layer in the CP in an 
inside-out manner. It means that late-born neurons migrate past already migrated deep layer neurons to be placed in the superficial (upper) cortical layers. The V/SVZ-derived excitatory neurons classically migrate radially into the CP. Radial neuronal migration can occur as somal translocation or locomotion (Angevine \& Sidman, 1961; Nadarajah et al., 2001; Kriegstein \& Noctor, 2004). During early stages of cortical formation, some early-born neurons migrate mainly by somal translocation, which entails the neurogenic differentiation of RGs leading to detachment of their apical disconnection followed by progressive or continual shortening of the basal processes (RG fibers) that results in upward movement and final placement of the somas (neurons) in the CP (Fig. 1.4A/B; (Miyata et al., 2001; Nadarajah et al., 2001)). Technically, except for the physical traction force provided by the basally anchored radial glial fiber, neurons migrating by somal translocation are not under the molecular guidance of the radial glial fiber (Nadarajah et al., 2001; Marin \& Rubenstein, 2003; Hawthorne et al., 2010).

A (Overview of radial migration)

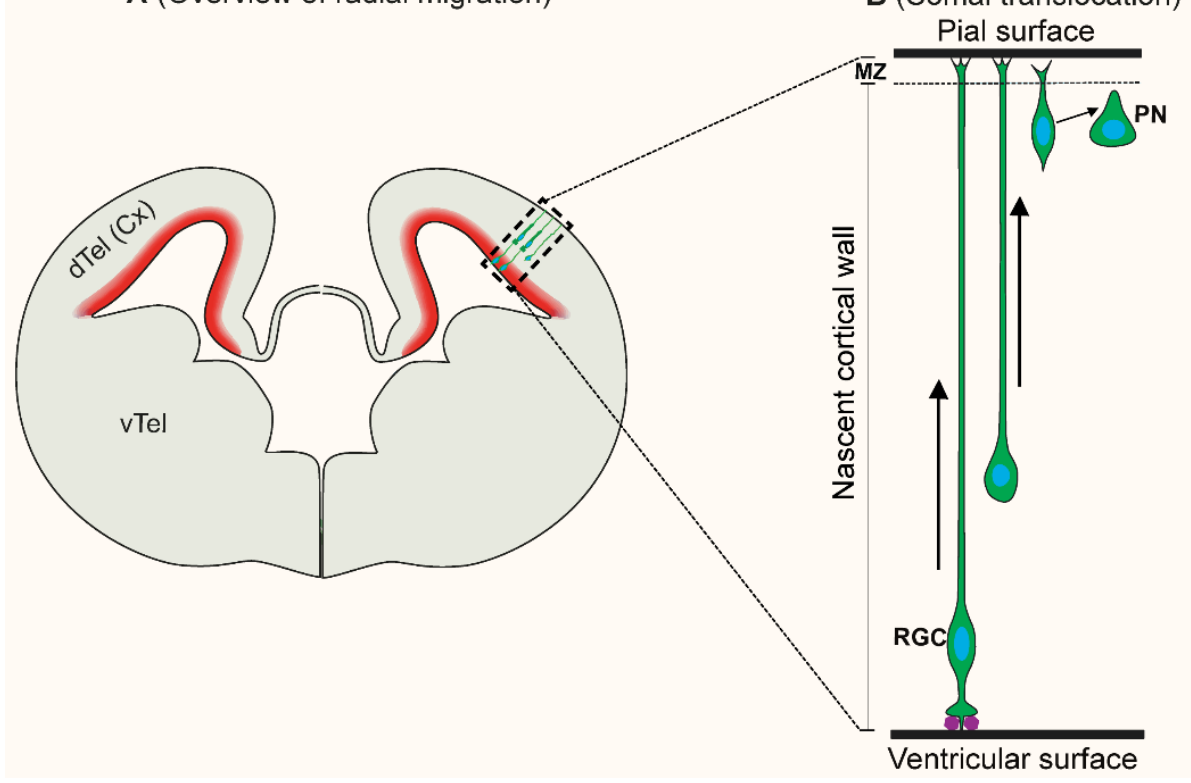

C (Locomotion)

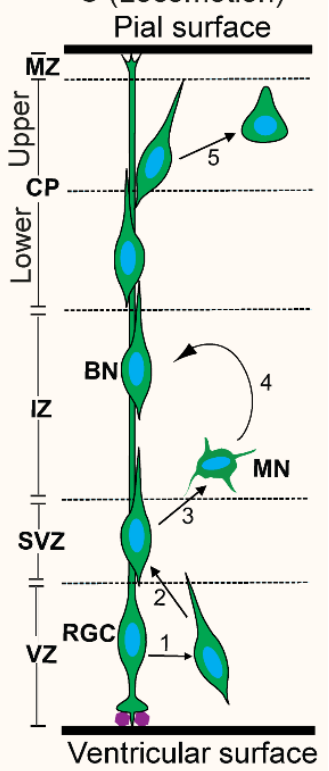

Figure 1.4 Schema showing modes of radial neuronal migration in the cortex: (A) Shows overview of the forms of glial-guided migration of neurons in the cortex (Cx). The ventricular zone is marked in red. (B) Zoom-in showing somal translocation of early-born projection/pyramidal neuron. The radial glial cell (RGC) disengages from the adherens junction belt (indicate in purple) at the ventricular surface and the soma is progressively moved upwards via shortening of the glial fiber length (depicted by upward 
vertical arrows) to be properly place in the nascent cortical wall or plate as differentiated projection neuron (PN). (C) Zoom-in showing the illustration of locomotion in the cortex. Locomotion is the mode of radial migration prominently adopted by late-born neurons. During locomotion the RGC maintains its apical and basal anchorage. After a RGC generates a neuron (step 1), the newborn neuron attaches to the glial fiber (step 2) and migrates out of the ventricular zone (VZ) into the subventricular zone (SVZ). It then detaches from the glial fiber on reaching the intermediate zone (IZ) and spends some time in the form of a multipolar neuron (MN) in the IZ (step 3). The MP neuron then switches morphology to a bipolar neuron (BN) and re-attaches to the glial fiber to resume locomotion (step 4) with a leading process facing pial surface and a trailing process directed towards the ventricular surface. In the cortical plate $(C P)$, the BN undergoes somal translocation to be final placed in its specified layer (step 5) when it makes contact with the marginal zone (Nadarajah et al., 2001; Noctor et al., 2004).

Locomotion on the other hand is a multimodal and challenging means of radial migration mainly used by later-born cortical neurons to exist their site of generation to the appropriate cortical laminar. The neurons migrating by locomotion use RG fiber molecular guidance cues such as cell-cell adhesion and radial orientation to reach their final destination in the CP. Locomoting neurons have bipolar morphology with a leading process (future dendrite) facing the pial surface and a trailing process in the direction of the VZ which becomes the future axon. They also exhibit a stationary phase during their long radial journey which is not seen in somal translocation (Fig. 1.4A/C; (Noctor et al., 2004; Ayala et al., 2007; Hatanaka \& Yamauchi, 2013)). Generally, neurons migrate by locomotion using the following key mechanics: formation and elongation of a leading process, neucleokinesis, and retraction of the trailing process (Marin et al., 2010). As cartooned in Figure 1.4C, the newly born neuron first migrates out the V/SVZ using the radial glial scaffold, cytoskeletal remodeling, and cell adhesion dynamics. When it reaches the border between the SVZ and IZ, it detaches from the glial fiber, acquires a multipolar morphology, and pauses migration to briefly stay in the IZ. The stationary phase in the $\mathrm{IZ}$ is believed to be important for collection of molecular instructions that ensure acquisition of proper orientation and timing signals for further migration. After sufficient molecular conditioning in the $\mathrm{IZ}$ and synaptic contacts with subplate cells, the multipolar neuron then reverses to bipolar morphology and reattaches to the RG fiber to continue locomotion into the CP (Noctor et al., 2004; Ayala 
et al., 2007; Mizutani, 2018; Ohtaka-Maruyama et al., 2018). Locomotion ends with a final stage of somal translocation when the leading process of the radially migrating neuron makes contact with the MZ leading to final detachment from the glial scaffold and placement of the soma in its designated position. Late-born neurons normally locomote past migrated early-born neurons such that cortical lamination is largely inverted. That is, the first cohort of cortical neurons form L6 located close to the ventricular surface, while the latest-born neurons form L2/3 located under L1 (Nadarajah et al., 2001; Kriegstein \& Noctor, 2004).

\subsection{Dysregulation of neuronal migration leads to abnormal cortical lamination and formation}

A plethora of factors are reported to regulate the generation, migration and final placement of cortical neurons in the developing cortex. Specific transcriptional factors in the neuronal transcriptome, signaling pathways, and epigenetic programs have been linked to the normal progression of radial neuronal migration to grant correct cortical cytoarchitectonics and axonal pathfinding (Kwan et al., 2012; Evsyukova et al., 2013). Notably, transcription factors like the T-box brain factor 1 (TBR1), fez family zinc finger 2 (FEZF2), and sex determining region Y-box 5 (SOX5) control migration of lower layer neurons to form deep cortical laminae (L6 and L5) (Hevner et al., 2001; Kwan et al., 2008; Lai et al., 2008; Han et al., 2011). On the other hand, the cell leukemia/lymphoma 11a (BCL11B/CTIP2), special AT-rich sequence binding protein 2 (SATB2), and the POU domain transcription factors POU3F2 (BRN2) and POU3F3 (BRN1), regulate positioning of late-born neurons during superficial cortical laminae formation (McEvilly et al., 2002; Sugitani et al., 2002; Alcamo et al., 2008; Britanova et al., 2008; Wiegreffe et al., 2015). 
Many factors involved in intracellular signal transduction have also been shown to play important roles in regulating radial migration of cortical neurons and related layer formation. Signaling cascades such as the Mitogen-activated protein kinase (MARK), Epidermal growth factor receptor (EGFR), RHO, REELIN, Fibroblast growth factor (FGF), NOTCH, Bone morphogenetic protein (BMP), and Wingless/Int (WNT) pathways are known to guide newly generated cortical neurons from their place of birth to their layer destination. Subtle perturbation of such signaling pathways can trigger observable alterations in cortical neuronal migration and positioning (reviewed in (Harrison-Uy \& Pleasure, 2012; Evsyukova et al., 2013). The WNT signaling in particular is a formidable regulator of cortical layer formation. Early activation of WNT signaling in neural progenitors resulted in loss of upper layer formation (Wrobel et al., 2007). Similarly, WNT activation at mid-corticogenesis preferentially disrupted upper cortical layer formation (Mutch et al., 2010). A more mechanistic insight was provided by a recent study which showed that transient downregulation of WNT signaling during the multipolar phase of radial neuronal migration is necessary to condition multipolar neurons to adopt bipolar morphology suitable for locomotion (Boitard et al., 2015).

The emerging role of epigenetic factors in neural development is also been realized in the orchestration of oriented-neuronal migration during cortical development. Activity of some micro RNAs (Delaloy et al., 2010; Clovis et al., 2012), and a component of the RNA biogenesis machinery, Dicer (Shibata et al., 2008; McLoughlin et al., 2012; Baudet et al., 2013), have been reported to play key roles during radial migration of cortical neurons. Likewise, dysregulation of the histone methyltransferase enhancer of zest homolog 2 (Ezh2), a subunit of the Polycomb repressor complex 2 (PRC2), perturbed neuronal orientation during radial migration (Zhao et al., 2015). Interestingly, 
depletion of a corepressor of chromatin remodeling complexes (CoREST), resulted in abnormal radial migration due to defective multipolar-to-bipolar transition of migrating cortical neurons (Fuentes et al., 2012). Hence, it is not surprising that chromatin remodelers themselves are important regulators of radial neuronal migration during cortical histogenesis. At least, this is known for some ATP-dependent chromatin remodeling complexes. For instance, the nucleosome remodeling deacetylase NuRD complex and chromodomain-helicase-DNA binding protein 8 (CHD8) have been implication in cortical neuronal migration anomalies (Nitarska et al., 2016; Xu et al., 2018). Similarly, Brm and Ctip1, a core and variable component of the BAF complex, respectively, have also been reported to instruct migration of cortical neurons. It was shown that in the absence histone deacetylase 2 (HDAC2) S-nitrosylation, Brm expression is impaired leading to disturbance of radial migration in the cortex (Nott et al., 2013). Ctip1 on the other hand negatively regulates the transcription of the semaphorin Sema3c to permit proper multipolar to bipolar morphology switch during radial migration (Wiegreffe et al., 2015).

Frequently, abnormal neuronal migration engenders various structural and function anomalies of the developing mammalian cortex which results in several syndromic and non-syndromic neurodevelopmental disorders (Evsyukova et al., 2013). By way of synopsis, various modes of neuronal migration aberration imputable to molecular dysregulation during development of the cortex typically culminate in phenotypes such as: (i) delayed migration or complete neuronal immotility, (ii) neuronal polarization/differentiation incompetence, (iii) delayed or absolute abolishment of multipolar-to-bipolar transition, (iv) misplacement of neurons leading to cortical mislamination, (v) ectopic nodular aggregation of neurons, (vi) abnormal projection of neuronal axons (tracts), (vii) over migration of neurons, (viii) neuronal apoptosis, (ix) 
abnormal neuronal circuitry, $(\mathrm{x})$ loss of cortical regionalization and/or arealization, and (xi) focal or global cortical hypoplasia. These and many other concurring histologic disturbances can have an overall devastating effect on normal cortical (brain) function; which can clinically present as cognitive or intellectual deficits and/or loss of sensorimotor functions (reviewed in (Evsyukova et al., 2013)).

\subsection{The BAF complex and its involvement in neural development}

The $\mathrm{Brg} 1 / \mathrm{Brm}$ associated factor (BAF) complex is a prominent chromatin regulator known to affect many aspects of cell biological processes mainly because of its involvement in the modulation of many epigenetic mechanisms. Emerging investigations have identified the BAF complex to play critical roles during embryonic and postnatal neural development. As such, many of its specific roles during brain morphogenesis have been substantially elucidated (Sokpor et al., 2017; Sokpor et al., 2018).

Despite its neurodevelopment regulation prowess, the BAF complex is, however, amenable to dysregulation that can result in abnormal modification of chromatin conformation which is capable of causing neurodevelopmental disturbances. Indeed, several syndromic and non-syndromic neurological disorders have been ascribed to mutations or mis-regulation of BAF complex subunits (reviewed in (Sokpor et al., 2017)). This has made many biologists and clinicians to be interested in dissecting the mechanisms through which the BAF complex acts to orchestrate neural development so as to unravel potential remedies for related neurodevelopmental anomalies.

\subsubsection{Biochemical properties of chromatin remodeling BAF (SWI/SNF) complex}

The SWI/SNF (SWItch/Sucrose Non-Fermentable) complex is a multi-protein complex known to be important in many cell biological processes in health or disease, and 
during evolution (Son \& Crabtree, 2014; Kadoch et al., 2016; Lu \& Allis, 2017; Sokpor et al., 2017; Stiller et al., 2018; Alfert et al., 2019). The BAF or mammalian (m)SWI/SNF complex, unlike the yeast version, is bulkier ( 2 MDa) because it is composed of many more subunits (at least 15) in vivo (Neigeborn \& Carlson, 1984; Wang, WD et al., 1996; Lessard et al., 2007; Wu et al., 2007). Generally, the BAF complex functions as a chromatin remodeler through the expenditure of energy obtained from the hydrolysis of ATP to mobilize parts of the nucleosome or the whole nucleosomal unit leading to increased accessibility of transcription or epigenetic factors to the genomic domains of chromatin and ultimate regulation of gene expression (Cairns, 1998; Phelan et al., 1999; Whitehouse et al., 1999; Gong et al., 2006; Gutierrez et al., 2007; Ho et al., 2009a; Tang et al., 2010; Hu et al., 2011; Tolstorukov et al., 2013). Together with the PRC2, the BAF complex regulate the fluidity of chromatin state via regulating the stoichiometry of heterochromatin and/or euchromatin signatures that translate into the establishment of gene expression modalities (Hara \& Sancar, 2002; Gong et al., 2006; Ho et al., 2009a; Ho et al., 2011; Hu et al., 2011; Tolstorukov et al., 2013; Nguyen et al., 2016; Kadoch et al., 2017; Nguyen et al., 2018).

The aforementioned ATPase activity is inherent to the BAF complex because it possesses exchangeable ATPases, namely Brahma (BRM) and BRM/SWI2 related gene 1 (BRG1), which are known to be core subunits of the complex (Neigeborn \& Carlson, 1984; Wang, WD et al., 1996; Lessard et al., 2007; Wu et al., 2007; Kadoch et al., 2013; Narayanan et al., 2015; Bachmann et al., 2016). The other characterized core subunits include two scaffolding subunits BAFF155 and BAF170, which are indispensable for the stable assembly of the other subunits in the complex (Fig. 1.5A/B; (Phelan et al., 1999; Chen \& Archer, 2005; Narayanan et al., 2015; Bachmann et al., 2016; Nguyen et al., 2016; Nguyen et al., 2018)). Many of the remaining subunits are 
variably incorporated into the complex depending on their biochemical demand in the cell. See Sokpor et al. (2017) for a comprehensive list of known BAF complex subunits. Together with the core subunits, these variant subunits can be assembled in combinatorial fashion or substitution of some subunits for their paralogs/isoforms to yield hundreds of functionally distinct BAF complexes that exhibit some cell type specificity. For instance, npBAF complex is only found in neural progenitors, and nBAF complex is exclusive to postmitotic neurons (Fig. 1.5A, (Lessard et al., 2007; Wu et al., 2007; Kadoch et al., 2013; Tuoc et al., 2013)). Recently, the olfactory neural stem cell (onsc)BAF and olfactory neuron (on)BAF complexes were reported to be operational in the olfactory neuroepithelium (Bachmann et al., 2016).

Apart from altering chromatin conformation, another possible function that unifies the various cellular types of the SWI/SNF (BAF) complexes is their role in maintaining lineage-specific enhancers and promoters (Ho et al., 2009b; Ho et al., 2011; Bossen et al., 2015; Barutcu et al., 2016; Alver et al., 2017; Wang et al., 2017). The chromatinbinding capacity of the BAF complex is afforded by the several DNA- and histonebinding motifs possessed by some of the subunits in the complex (reviewed in (Sokpor et al., 2017)).

\subsubsection{The multi-functional role of BAF complex in neural development}

The existence of several neurodevelopment and neuropsychiatric disorders consequent to specific mutations or genetic ablations of BAF complex subunits is reflective of the indispensability of the BAF complex in neural tissues. Indeed, a host of investigations have implicated various components and/or the entire BAF complex functionality to be involved in the regulation of several aspect of neural development, including neural progenitor specification and proliferation, neurogenic/gliogenic 
differentiation, neuronal maturation, and synaptogenesis (Fig. 1.6; (Sokpor et al., 2017)).

A

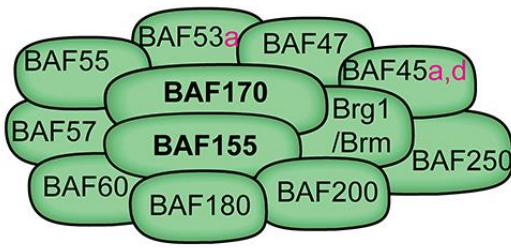

npBAF complex

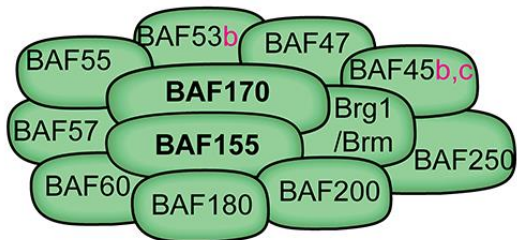

nBAF complex
B BAF complex inactivation

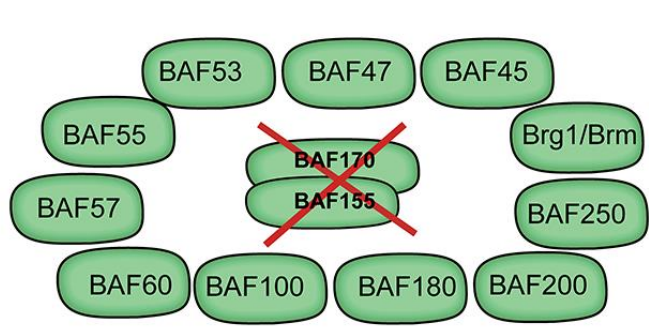

BAF155/BAF170 dcKO

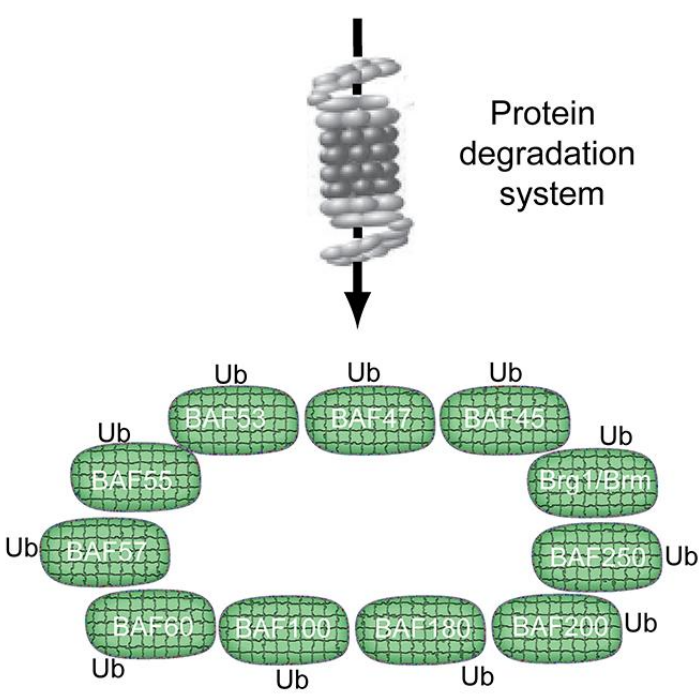

BAF subunit degradation

Figure 1.5 Models showing the known forms of BAF complexes in neural cells and genetic scheme for entire BAF complex knockout: (A) Image shows a cartoon of the BAF complex reported to be found only in neural progenitors (npBAF complex). The npBAF is especially distinguished by the following BAF subunits: BAF53a, BAF45a and BAF45d among others as indicated. The BAF complex found in neurons (nBAF complex) is formed when the said BAF subunits are respectively replaced by their isoforms as indicated i.e. BAF53b, BAF45b and BAF45c (Lessard et al., 2007; Wu et al., 2007; Bachmann et al., 2016). (B) Illustration shown that double deletion of the BAF complex subunits BAF155 and BAF170 leads to disassembly of the other subunits which then become targeted by the ubiquitin (Ub) proteasome complex leading to their elimination via degradation. Red cross denotes deletion (Narayanan et al., 2015).

What then makes the BAF complex have such neurodevelopmental plurality and specificity? First, the BAF complex is ubiquitously expression in the body with demonstrable prominence in the central nervous system (Smith-Roe \& Bultman, 2013; Alexander et al., 2015; Nguyen et al., 2016). 


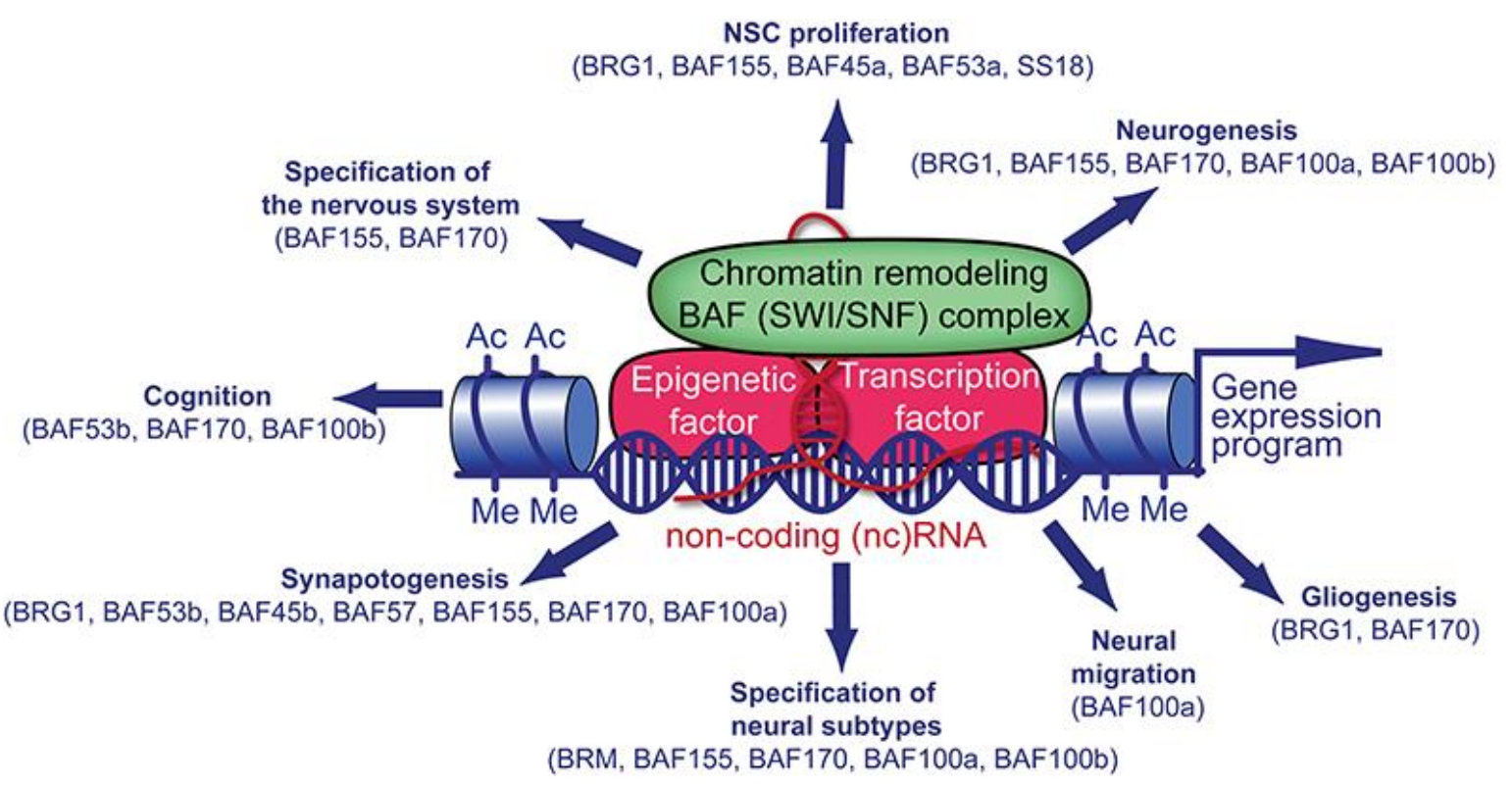

Figure 1.6 Schema showing overview of the multiple neurodevelopmental functions of the BAF (mSWI/SNF) complex: The BAF complex, epigenetic factors (including non-coding [nc] RNA), and transcription factors control gene expression. Transcription factors and ncRNAs recognize and bind to specific DNA sequences. The recruitment of BAF complex and other epigenetic factors to the genome leads to alteration of epigenetic marks (e.g., histone methylation, Me; histone acetylation, Ac) and chromatin conformation in order to activate or repress a specific gene expression program in neural cell lineages. Many BAF complex subunits as indicated, have been associated to the regulation of specific processes during neural development. NSC; neural stem cell (Sokpor et al., 2017).

Second, BAF complex may be uniquely assigned to the establishment of specific chromatin landscape(s) to elicit particular neurodevelopmental transcriptional programs different from those orchestrated by other chromatin remodelers (Sokpor et al., 2018). Third, a possible interplay between BAF complex and other powerful signaling determinants of neural development may exist. A notable means by which BAF complex likely establishes its effect on neural genesis is through its interaction with factors or targets of the WNT (Barker et al., 2001; Vasileiou et al., 2015), Notch, and Sonic hedgehog (Zhan et al., 2011) signaling cascades, which are prominent pathways implicated in the regulation of many developmental processes, including patterning. During neural development, the BAF complex controls WNT signaling in a bimodal manner, i.e. by inhibition or activation. However, its opposing counterpart, the PRC2, antagonizes WNT signaling or its targets to establish a homeostatic balance 
between the rate of progenitor proliferation and neurogenic fate acquisition (Barker et al., 2001; Hirabayashi \& Gotoh, 2010; Ronan et al., 2013; Vasileiou et al., 2015; Nguyen et al., 2018).

\subsubsection{Role of BAF complex in neural progenitor specification and proliferation}

The specification and patterning of neural cells/tissues is central to the proper morphogenesis of the nervous system. The BAF complex has been identified as a possible player in the specification of naïve cells (i.e., embryonic stem cells) to acquire neural fate during development (Ho et al., 2009a; Ho et al., 2009b; Hirabayashi \& Gotoh, 2010; Juliandi et al., 2010; Coskun et al., 2012; Ronan et al., 2013; Yao et al., 2016). Accordingly, it was shown that complete abrogation of BAF complex under Foxg1-Cre driver in the early developing brain led to partial or incomplete specification of telencephalic tissues (Narayanan et al., 2015; Nguyen et al., 2016). Likewise, the olfactory neuroepithelium in such BAF complex mutants was not specified (Bachmann et al., 2016). Invariably, the early deletion of BAF complex hampered overall development leading to early embryonic lethality (Nguyen et al., 2016).

Neural progenitor or stem cells are the first cohort of cells to be established in the properly specified neuroepithelium. To increase their pool and reserves for later expansive development, neural progenitors must self-renew and/or proliferate. The BAF complex has been reported to play key roles in directing proliferation of neural progenitors and the temporal switch to differentiative mode during neurodevelopment. To initiate proliferation and maintain multipotency in neural progenitors, the derivation of npBAF complex from the embryonic stem cell (esc)BAF complex is required (Lessard et al., 2007; Staahl et al., 2013; Bachmann et al., 2016). In vitro evidence alongside studies in worms and rodents have shown that the BAF complex subunits, 
including Brg1 (psa-4, in worm), BAF155 (psa-4, in worm), BAF170, BAF45a/d, and BAF53a (Bultman et al., 2000; Kim et al., 2001; Matsumoto et al., 2006; Lessard et al., 2007) regulate neural cell proliferation. Recently, it was shown that partial ablation or complete inactivation of BAF complex resulted in increased neural progenitor cell proliferation at the expenses of differentiation which resulted in perturbation of cortical neurogenesis and formation (Nguyen et al., 2018; Holdhof et al., 2019). Fully primed neurogenic progenitors were reported to acquire or reverse to early neuroepithelial progenitor fate following ablation of BAF complex in the forebrain at mid-stage corticogenesis (Nguyen et al., 2018). These recent findings consolidate the importance of BAF complex as an epigenetic regulator of neural progenitor cell proliferation and specification.

\subsubsection{BAF complex regulates neuronal differentiation and subtypes generation}

After the multipotent neural progenitor cell pool has been adequately established in the germinal zones of say the embryonic forebrain, many intrinsic and extrinsic factors act as differentiative cues to stimulate acquisition of unipotent fate which ultimately leads to neural progenitors differentiating into neurons (Guillemot, 2007; Kriegstein \& Alvarez-Buylla, 2009; Taverna et al., 2014). The need for such neurogenic differentiative schemes calls for alterations in the epigenetic milieu as well. Chromatin remodelers are notable regulators of such epigenetic modifications in neural stem cells leading to their differentiation (Juliandi et al., 2010). In the case of the BAF complex, there is competition in subunit dosage integration and isoform substitution leading to re-composition of the npBAF to form $\mathrm{nBAF}$ complex (Fig. 1.5A) which is mechanistically partly mediated by the transcription factors Tbr1, Tbr2, and Pax6, and some microRNAs (Lessard et al., 2007; Wu et al., 2007; Tuoc et al., 2013; Bachmann 
et al., 2016; Elsen et al., 2018). The BAF complex has been shown to play a decisive role in the adoption of direct versus indirect neurogenesis (Sawa et al., 2000; Tuoc et al., 2013), where the latter is favored over the former to promoted cortical expansion via promotion of basal progenitor cell generation. During cortical development, the BAF complex can repress Pax6 targets leading to the predominance of direct over indirect neurogenesis by increasing dosage of BAF170 in the complex. However, to switch to indirect neurogenic mode, BAF155 dosage is increased at the expense of BAF170 (Tuoc et al., 2013). Interestingly when Brg1 is loss in the BAF complex, the neuronal differentiation program is blocked in favor of gliogenic differentiation which is phenocopied in the Pax6-ablated cortex (Ninkovic et al., 2013; Petrik et al., 2015).

For the most part, the capacity to generate neurochemical and functional distinctions among the population of neurons that comprise the cortical laminae during brain development may be inherently encoded in cortical neuronal progenitors and modulated epigenetically at the postnatal stages of cortical development (Greig et al., 2013; Harb et al., 2016). There is compelling evidence indicating that the chromatin remodeling BAF complex is essential for establishing neuronal diversity programs that lead to the generation of the various neuronal subtypes during cortical neurogenesis. Notable among them is the requirement of the dual role of BAF155 and BAF170 in switching on the gene expression programs that confer upper layer neuronal identity at late embryonic neocortical development (Tuoc et al., 2013). BAF100a (Bcl11a/Ctip1) and BAF100b (Bcl11b/Ctip2) have been shown to also play critical roles in the determination of subclass identity of the many projection neurons generated during corticogenesis. Detailed mechanisms involved are reviewed in Sokpor et al., (2017). 


\subsubsection{Neuronal migration, maturation, and synaptogenesis are regulated by}

\section{BAF complex}

Immature postmitotic neurons undergo some developmental changes culminating in their morphological differentiation that contributes to their normal migration and functional maturation. As previously mentioned, some subunits of the BAF complex may cooperate with other factors to regulate neuronal migration (Nott et al., 2013; Wiegreffe et al., 2015). The properly migrating/migrated neurons further undergo structural and functional differentiation to become mature. Important among such maturation processes are the elaboration of axons (axonogenesis), and the development of dendrites (dendritogenesis) needed for the formation of synapses. Dysregulation of neuronal axonogenesis and dendritogenesis underpins many neurodevelopmental anomalies. Chromatin remodeling factors contribute to the molecular regulation of neuronal axon formation, pathfinding, and dendritic outgrowth and refinement (Whitford et al., 2002; Wu et al., 2007; Bachmann et al., 2016).

The BAF complex is known to take part in the regulation of the morphological and functional maturation of neurons during neural tissue morphogenesis largely through control of the selection of neurites that will differentiate to become axons or dendrites (Whitford et al., 2002; Wu et al., 2007; John et al., 2012; Weinberg et al., 2013; Bachmann et al., 2016). Loss of the subunits in the neuron-specific BAF complex such as Brg1, BAF45b, BAF53b (or BAP55 in fly), and BAF57 disturbs activity-dependent dendritic development both in vitro and in vivo (Parrish et al., 2006; Lessard et al., 2007; Wu et al., 2007; Tea \& Luo, 2011). Mice with homozygous loss of say BAF53b present with behavioral problems (Lessard et al., 2007; Wu et al., 2007). Mechanistically, the BAF complex may regulate dendrites formation possibly by 
remodeling the promoter regions of genes (e.g., GAP43 and Ephexin1) involved in dendritogenesis needed for neuronal circuit formation (Wu et al., 2007). It has also been proposed that the BAF complex may mechanistically engage in activity responsive $\mathrm{Ca}^{2+}$ signaling via its interaction with the transcriptional activation function of calcium-responsive transactivator (CREST) known to instruct dendritic morphodifferentiation of neurons (Aizawa et al., 2004; Wu et al., 2007).

\subsubsection{Genesis of glial cells may be controlled by BAF complex function}

After sufficient neurons are generated during neurogenesis, the neurogenic RGs switch fate to become glia cell-generating progenitors (Gage, 2000; Freeman, 2010; Rowitch \& Kriegstein, 2010). The generation of astrocytes (astrogenesis), and oligodendrocytes (oligodendrogensis) have received much attention during development of the central nervous system (Rowitch \& Kriegstein, 2010; Gallo \& Deneen, 2014). Transcriptional and epigenetic programs, including chromatin remodeling have been implicated in the transition from neurogenesis to gliogenesis (Hirabayashi \& Gotoh, 2010; Juliandi et al., 2010; Coskun et al., 2012; Yao et al., 2016). The BAF complex appears to perform important role in cortical gliogenesis. When Brg1 or BAF170 are ablated, in the developing or early postnatal brain, there is precocious transition from neurogenic to gliogenic differentiation leading to early depletion of the neural progenitor cells pool (Matsumoto et al., 2006; Lessard et al., 2007; Ninkovic et al., 2013; Tuoc et al., 2017).

During the generation of oligodendrocytes in the cortex, the Brg1-contain BAF complex has been identified to cooperate with the transcription factor Olig2 to drive the expression of genes that promote oligodendrogenesis. As a result, Nestin-mediated inactivation of BRG1 during early development of the brain resulted in premature 
expression of Olig2+ cells that were anyhow incapable of generating viable oligodendrocytes, if any at all (Matsumoto et al., 2006; Yu et al., 2013; Bischof et al., 2015). It implies that the BAF complex may function as an epigenetic factor that represses gliogenic tendencies in the neural progenitor cell during early stages of corticogenesis. It is not far-fetched to conceive a possible role of BAF complex in astrogenesis since astrocytes or their precursors share common early progenitor cell ancestry with oligodendrocytes generated within the cortex.

\subsection{Emerging role of epigenetic factors in pre-patterning during corticogenesis}

The emergence of the idea of epigenetic pre-patterning is extending the frontiers of molecular regulation of brain patterning. Pre-patterning is the phenomenon in which a repertoire of lineage-specific genes that are transcriptionally switched off become temporally poised for later activation by specific regulatory factors including chromatin modifiers and remodelers, especially in multipotent cells (Xu \& Zaret, 2012; Chen \& Dent, 2014). The theme epigenetic pre-patterning is thus gradually gaining attention in the area of cortical development (Albert \& Huttner, 2018; Yoon et al., 2018). Neural progenitor/stem cells that are located in the VZ or SVZ and generate the diverse projection neurons that migrate radially to form the cortical layers may be pre-patterned such that proneural genes that confer neuronal identity are silenced until the appropriated differentiation stage. Alterations in the epigenetic landscape is a plausible means by which pre-patterning of neural progenitors is effected (Mohn et al., 2008; Ziller et al., 2015).

A key evidence indicating that neural progenitors are pre-patterned (primed) is that some neuronal genes are already expressed at low levels in a selected population of progenitors in the cortical germinal zones. For instance, Cux1/2 and Satb2 genes that 
are highly expressed in upper layer cortical neurons are also expressed in some cortical progenitors (Nieto et al., 2004; Franco et al., 2012). Similarly, some lower layer neuron-specifying genes are expressed in a subset of APs in the developing cortex (Frantz et al., 1994; Molyneaux et al., 2005). It implies that abnormal pre-patterning may have detrimental consequences on the differentiation, placement (migration) and functional maturation of progenies of neural progenitors during cortical development.

Compelling evidence pointing to the emerging influential role of epigenetic factors in cortical neural progenitor pre-patterning include the identification of the PRC2 as a regulator of the temporal progression of APs in the developing cortex (Telley et al., 2019), and the effect of ablation of the chromatin remodeling BAF complex on cortical neurogenesis (Tuoc et al., 2013; Nguyen et al., 2018). The PRC2 was found to control the successive transmission of the molecular birthmarks from APs to their postmitotic progenies in a ground state that is put into full force by external differentiative cues (Telley et al., 2019). Intriguingly, the BAF complex, which acts in opposition to the PRC2, was reported to regulate the proper generation of the upper and lower layer neurons from neural progenitors in the developing cortex by controlling chromatin state permissive for Pax6 transcriptional activity (Tuoc et al., 2013; Narayanan et al., 2015; Narayanan et al., 2018; Nguyen et al., 2018). As a result, in the absence of BAF170 there is over production of intermediate neuronal precursors fated to produce superficial cortical layer neurons. However, overexpression of BAF170 in the developing cortex leads to the depletion of such upper layer neurons (Tuoc et al., 2013). Deletion of BAF155, a paralog BAF170, probably resulted in abnormal priming of APs leading to potentiation of BP generation without commensurate increase in neuronal output during corticogenesis (Narayanan et al., 2018). Interestingly, the complete inactivation of BAF complex via double deletion of BAF155 and BAF170 in 
embryonic neural progenitors resulted in severe impairment of neurogenesis leading to diminished generation of upper and lower layer neurons (Nguyen et al., 2018). The BAF complex may also act epitranscriptomically through interaction with RBM15, a component of RNA methylation machinery, to regulate pre-patterning of cortical progenitors during forebrain development (Xie et al., 2019).

\subsection{General objectives of the studies}

The various specific objectives of the studies presented in chapters 2-4 were collectively aimed at further dissecting the roles of the chromatin remodeling BAF complex in orchestrating development of the mammalian cerebral cortex. Specifically, the investigations in chapter 2 aimed at providing further insight into how the BAF complex stability is maintained by its subunits BAF155 and BAF170 and the consequence of their ablation in the epigenetic regulation of cortical development. In chapter 3, the aim was to determine if and how the BAF complex is involved in regulating very early cortical developmental events that lead to the specification and subdivision of the cortical primordium during patterning of the cortex. The work in chapter 4 was essentially aimed at unraveling the involvement of BAF complex in the placement of cortical neurons via the mechanistic regulation of radial migration to afford proper layer organization during cortical organogenesis. 
CHAPTER 2: Epigenetic regulation by BAF (mSWI/SNF) chromatin remodeling complexes is indispensable for embryonic development

All elements in this section have already been published in:

Nguyen $\mathrm{H}^{*}$, Sokpor $\mathrm{G}^{*}$, Pham L, Rosenbusch J, Stoykova A, Staiger JF, Tuoc T. 2016. Epigenetic regulation by BAF ( $\mathrm{mSWI} / \mathrm{SNF}$ ) chromatin remodeling complexes is indispensable for embryonic development. Cell Cycle 15(10): 1317-1324.

* Authors with equal contribution.

Personal contributions: I was coequally involved in characterizing the dcKO phenotypes, data analyses, and drafting and finalization of the manuscript.

\subsection{Abstract}

The multi-subunit chromatin-remodeling SWI/SNF (known as BAF for Brg/Brmassociated factor) complexes play essential roles in development. Studies have shown that the loss of individual BAF subunits often affects local chromatin structure and specific transcriptional programs. However, we do not fully understand how BAF complexes function in development because no animal mutant had been engineered to lack entire multi-subunit BAF complexes. Importantly, we recently reported that double conditional knock-out ( $d c K O)$ of the BAF155 and BAF170 core subunits in mice abolished the presence of the other BAF subunits in the developing cortex. The generated $d c K O$ mutant provides a novel and powerful tool for investigating how entire BAF complexes affect cortical development. Using this model, we found that BAF complexes globally control the key heterochromatin marks, H3K27me2 and -3 , by 
directly modulating the enzymatic activity of the H3K27 demethylases, Utx and Jmjd3. Here, we present further insights into how the scaffolding ability of the BAF155 and BAF170 core subunits maintains the stability of BAF complexes in the forebrain and throughout the embryo during development. Furthermore, we show that the loss of BAF complexes in the above-described model up-regulates H3K27me3 and impairs forebrain development and embryogenesis. These findings improve our understanding of epigenetic mechanisms and their modulation by the chromatin-remodeling SWI/SNF complexes that control embryonic development.

\subsection{Introduction}

Embryogenesis and organogenesis are determined by the combined effects of myriad developmental events. In recent years, we have made substantial advances in understanding how embryonic development is regulated (Ho \& Crabtree, 2011; Kojima et al., 2014). The early development are coordinated by different molecular programs, in which epigenetic and chromatin-related controls are known to play crucial roles (Ho \& Crabtree, 2011). Epigenetic regulation, which modulates the chromatin structure without altering the DNA sequence, has profoundly heritable influences on transcriptional programs (Heard \& Martienssen, 2014). These changes in chromatin organization activate or repress gene expression programs either globally or locally, and may thus shape specific developmental events. Epigenetic mechanisms and chromatin regulation influence the ability of transcription factors (TFs) to access regulatory elements in their target genes. This occurs primarily via histone modification (Goldberg et al., 2007) or the action of ATP-dependent chromatin remodeling complexes, such as SWI/SNF (BAF) complexes (Wen et al., 2009; MuhChyi et al., 2013; Narlikar et al., 2013; Ronan et al., 2013). In addition, recent studies have shown that 
DNA methylation (Wu \& Zhang, 2014) and long non-coding RNA (IncRNA)-based mechanisms (Bohmdorfer \& Wierzbicki, 2015) also contribute to the complexity of epigenetic regulation during development.

The types of covalent histone modification include histone acetylation, methylation, ubiquitination and phosphorylation (Strahl \& Allis, 2000; Goldberg et al., 2007). Histone modification (epigenetic marks) is catalyzed by two enzyme classes: histone writers (e.g., histone acetyltransferases, methyltransferases, kinases, and ubiquitin ligases) and histone erasers (e.g., histone deacetylases, demethylases, phosphatases, and deubiquitinases). The mis-regulation of histone writers and erasers will typically alter the epigenetic program and have profound effects on development (Strahl \& Allis, 2000; Goldberg et al., 2007).

A number of non-covalent, energy-dependent chromatin remodeling complexes modulate the dynamicity of chromatin structures. Among them, the SWI/SNF complexes are the best characterized in both development and disease. Mammalian SWI/SNF (BAF) complexes are made up of two switchable ATPase subunits (Brg1 or Brm), core subunits (BAF47, BAF155, and BAF170) and a variety of lineage-specific subunits (Lessard et al., 2007; Ho et al., 2009b; Kadoch et al., 2013; Ronan et al., 2013). The Brg1 and Brm ATPases hydrolyze adenosine triphosphate (ATP) and utilize the obtained energy to alter chromatin (nucleosome) structures, thereby modulating cellular processes such as gene expression (Hirschhorn et al., 1992; Laurent et al., 1993; Phelan et al., 1999). The various subunits (at least 15 have been identified) are capable of undergoing combinatorial assembly (Wang, W et al., 1996; Ronan et al., 2013), yielding hundreds of distinct BAF complexes that can direct specific transcriptional events during development in vivo. The exceptional diversity of 
BAF complexes allows them to have functional specificity in biological processes. To investigate the roles of BAF complexes in development, researchers have focused on phenotypic analyses of model animals harboring mutations in single BAF subunits (Ko et al., 2008; Ho \& Crabtree, 2011; Narayanan \& Tuoc, 2014). However, although BAF complexes are known to play essential roles in development, studies using knock-out mouse models for individual BAF subunits have yielded incomplete information regarding the functions of these complexes.

While the epigenetic machinery and chromatin-remodeling complexes are known to play essential roles in development, we know little about how they interact to coordinate developmental processes during embryogenesis and organogenesis. Recently, our group developed cortex-specific BAF155/BAF170cKO mouse mutant and showed that BAF complexes did not form in the cortices of these mice. We further showed that the known BAF subunits undergo proteasome-mediated degradation in the developing cortices of these mutants. Finally, we found that, during corticogenesis, BAF complexes globally control key heterochromatin marks (H3K27me2/3) by directly interacting with and modulating the enzymatic activity of the H3K27 demethylases. Here, we discuss these recent discoveries (Narayanan et al., 2015) and present additional evidence suggesting that the BAF155 and BAF170 core subunits cooperate to stabilize the BAF complex and maintain the global level of H3K27me3 both in the developing forebrain and throughout the embryo. Our new findings indicate that BAF complexes act as key regulators of embryogenesis. 


\subsection{Results and Discussion}

2.3.1 BAF155 and BAF170 are indispensable for brain development and embryogenesis

By employing cortex-specific conditional mouse mutagenesis, we showed that the dual loss of the BAF155 and BAF170 subunits in double conditional knock-out (dcKO) mutants severely perturbed the growth of cortical structures, blocked the proliferation, differentiation and cell-cycle progression of cortical progenitors, and triggered a massive increase in the number of apoptotic cells (Narayanan et al., 2015). To further investigate how the loss of both BAF155 and BAF170 affects forebrain development, we generated forebrain-specific BAF155 and BAF170 dcKO mice by crossing mice floxed for BAF155 (Choi et al., 2012) and BAF170 (Tuoc et al., 2013) (BAF155 fl/fl, $B A F 170^{f / f f}$ ) with a FoxG1-Cre line (Hebert \& McConnell, 2000). In FoxG1-Cre mice, the Cre-recombinase is driven in all telencephalic cells [including those of the cortex $(\mathrm{Cx})$ and basal ganglia (BG)], but not in other parts of the brain [e.g., in the diencephalon (Di)] (Hebert \& McConnell, 2000). Remarkably, we found that the dcKO_FoxG1-Cre mutants completely lacked all telencephalic structures at E16.5 (Narayanan et al., 2015). This indicated that the expressions of BAF155 and BAF170 are required for brain development. 
Ubiquitous-inducible CAG-Cre (TAM: E9.5)

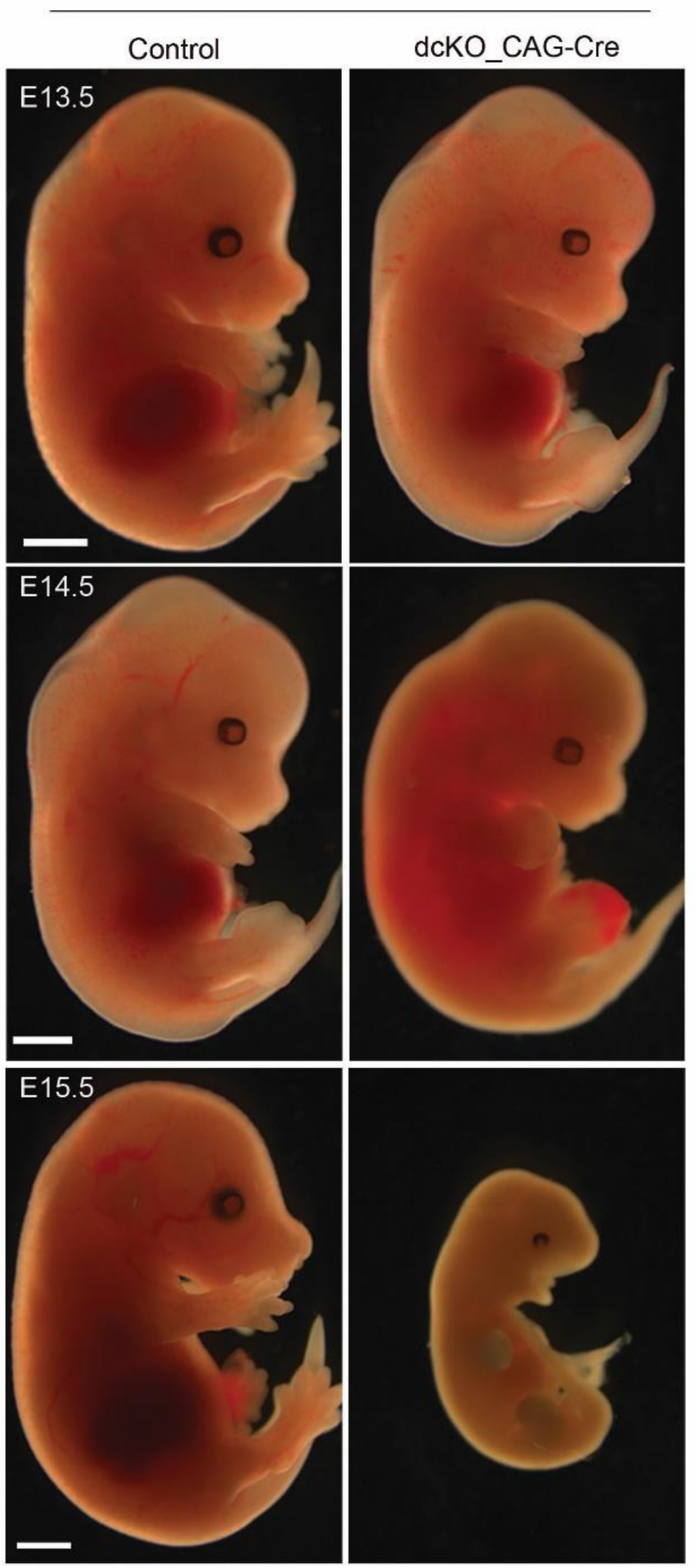

Figure 2.1 The expressions of BAF155 and BAF170 are indispensable for embryonic development: dCKO_CAG-Cre embryos treated with TAM at E9.5 remained alive and showed roughly preserved morphology at E13.5, but thereafter died between E14.5 and E15.5. Scale bars $=1000 \mu \mathrm{m}$. 
To address whether BAF155 and BAF170 are essential for embryogenesis, we generated and analyzed a line harboring a full dcKO_CAG-Cre mutant with the tamoxifen (TAM)inducible ubiquitous deleter, CAG-Cre line (Hayashi \& McMahon, 2002) (Fig. 2.1). The dcKO_CAG-Cre mutants were injected with either TAM or corn oil (vehicle solution, control) at E9.5. Following TAM induction, we observed Cre-recombinase activation in all cells of the body (Hayashi \& McMahon, 2002). The mutants died between E14.5E15.5, and exhibited a severe developmental retardation (Fig. 2.1). Together, these results show that the expressions of BAF155 and BAF170 are critical for determining overall embryogenesis, including the formations of the forebrain and cortex.

\subsubsection{BAF155 and BAF170 control the stability of BAF complexes in both cultured cells and embryos}

Hundreds of distinct BAF complexes are predicted to form in vivo by the combinatorial assembly of at least 15 identified BAF subunits (Ho \& Crabtree, 2011). The functional specificity of a BAF complex is believed to reflect the composite surfaces of its integrated subunits, which are essential for the ability of these complexes to target the genome and interact with transcriptional factors (TFs), co-activators, co-repressors, and signaling pathways (Ho \& Crabtree, 2011). We recently reported that BAF155 and BAF170 act as scaffolding subunits and are required to ensure the stability of the entire BAF complex in the developing cortex (Narayanan et al., 2015). The loss of BAF155 and BAF170 in cortex-specific dcKO mutants leads to the dissociation of all other BAF subunits from the complex. The free BAF subunits are subsequently ubiquitinated and degraded by the proteasome system. 
In an effort to extend our analysis to other parts of the brain, we examined the expression levels of various BAF subunits (e.g., Brg1, Brm, BAF47, BAF60, and BAF250) following the loss of BAF155/BAF170 in telencephalon of dcKO_FoxG1-Cre embryos (Fig. 2.2). Consistent with the Cre-recombinase activity in the $\mathrm{Cx}$ and BG of dcKO_FoxG1-Cre mice, there was no detectable expression of BAF155 or BAF170 in these structures. In contrast, their expression levels were preserved in the $\mathrm{Di}$, where Cre is inactive (Figure 2.2 A/B). Similar to the reported effects in cortical tissues (Narayanan et al., 2015), the loss of BAF155 and BAF170 in the telencephalon abrogated the expression of all BAF subunits throughout this structure, including in the BG (Fig. 2.2 C-G). To investigate whether both BAF155 and BAF170 are required to stabilize BAF complexes throughout the embryo, the expression of BAF subunits was examined in ubiquitously inducible dcKO_CAG-Cre embryos with global loss of BAF155/BAF170 (Fig. 2.3). These dcKO_CAG-Cre mutants were injected with either TAM or corn oil (vehicle solution, as control) at E9.5 and analyzed at E13.5, when the mutants were still viable. Following treatment with TAM, the expression levels of BAF155 and BAF170 were considerably ablated (Fig. 2.3A-D). Moreover, the expression levels of the tested BAF subunits (Brg1, Brm, BAF47, and BAF250) were severely diminished throughout the dcKO_CAG-Cre embryos, as compared to controls (Fig. 2.3E-L). These findings suggest that BAF155 and BAF170 are required to maintain the expression levels of BAF subunits in living animals. 


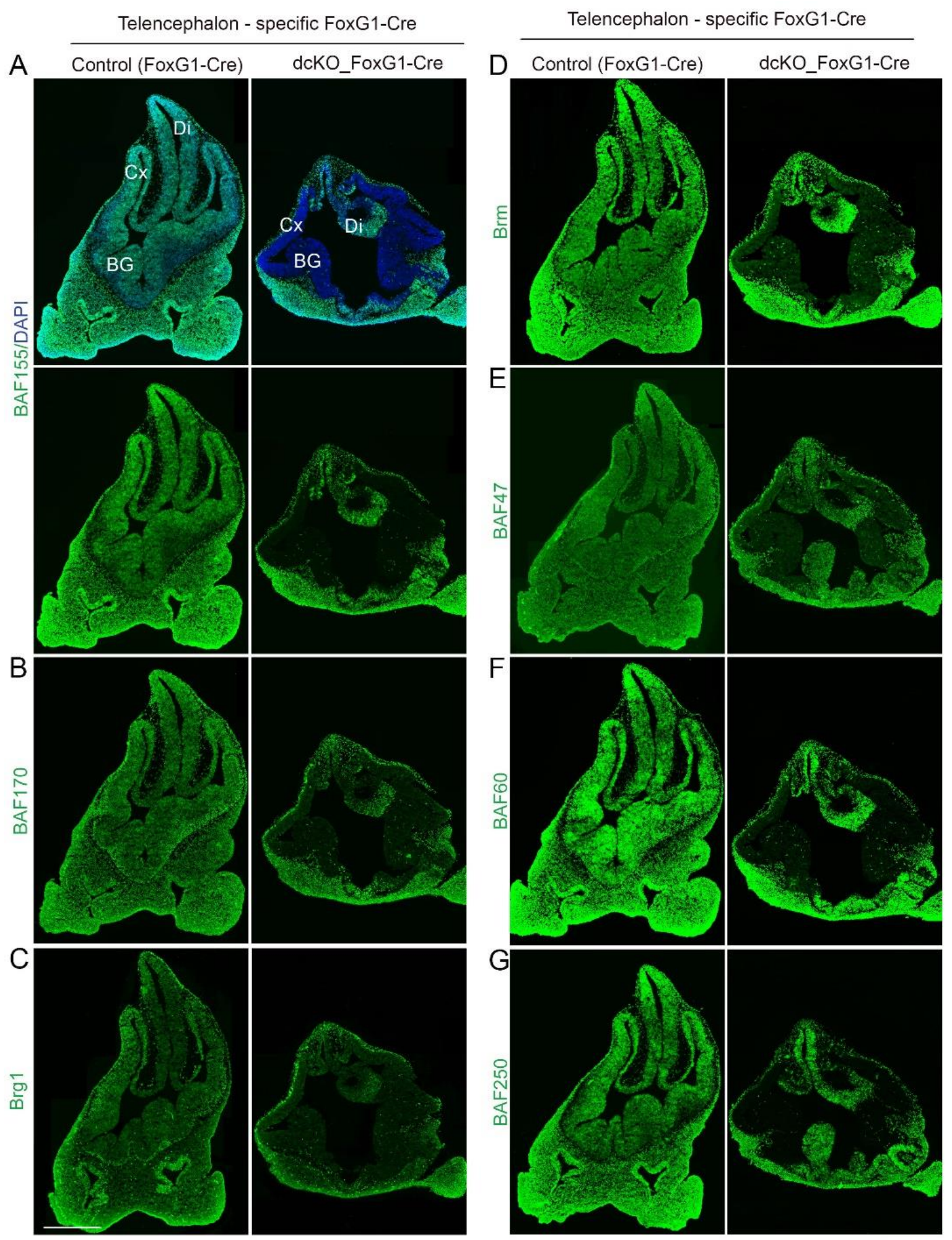

Figure 2.2 Expression of BAF subunits in telencephalon-specific dcKO_FoxG1-Cre mutants: (AG) Images show immunohistochemical (IHC) analyses for various core subunits of BAF complexes, including BAF155 (A), BAF170 (B), Brg1 (C), Brm (D), BAF47 (E), BAF60 (F), and BAF250 (G), in the forebrains of dcKO_FoxG1-Cre mutants at E11.5. The indicated BAF subunits are not detected in the BAF155/BAF170-knockout telencephalon. Scale bars $=500 \mu \mathrm{m}$. Abbreviations: Cx, cortex; BG, basal ganglia; and Di, diencephalon. 
E13.5 (TAM: E9.5)

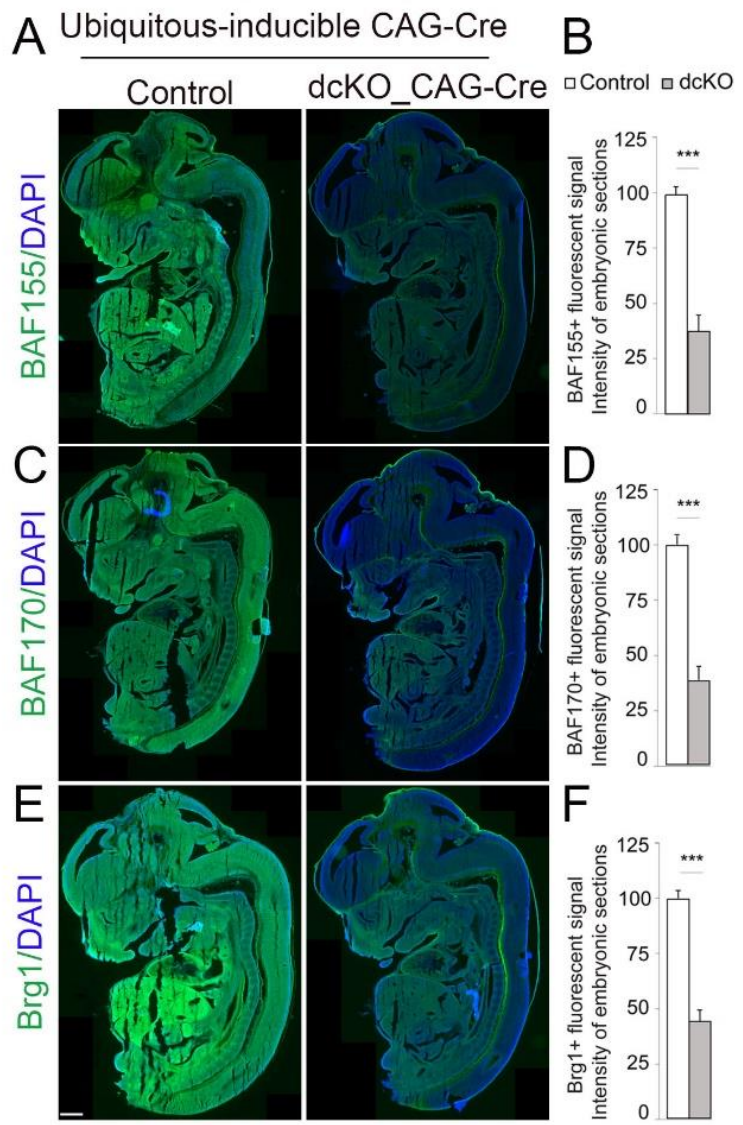

E13.5 (TAM: E9.5)

$\mathrm{G}$ Ubiquitous-inducible CAG-Cre $\mathrm{H}$

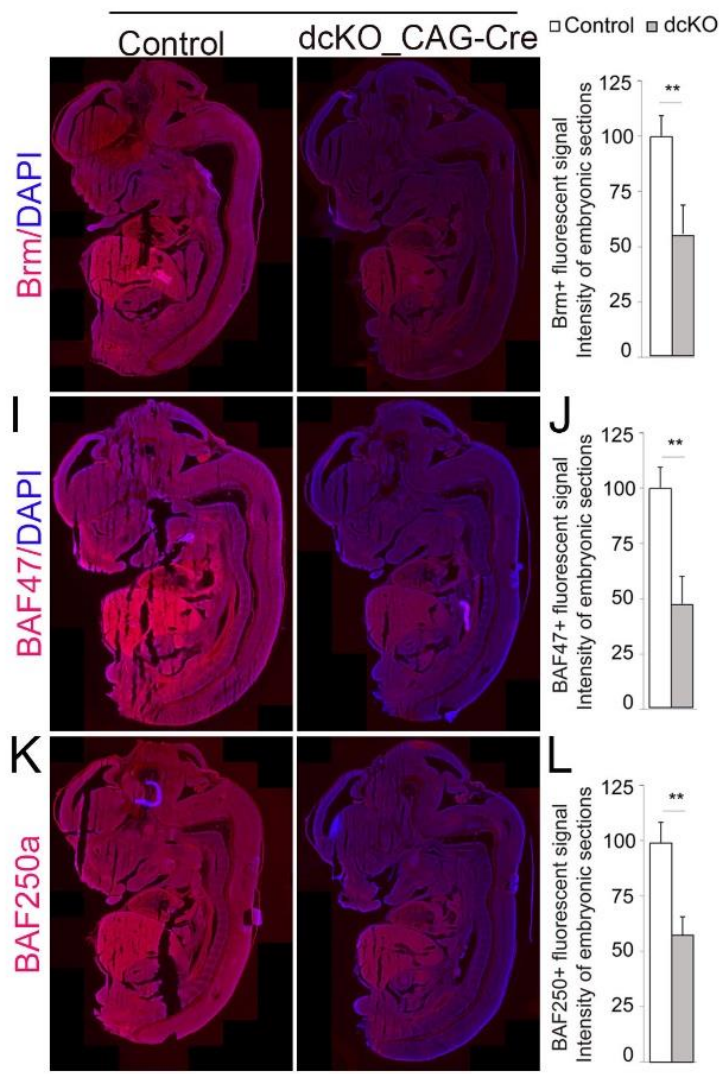

Figure 2.3 Expression of BAF subunits in embryos of TAM-inducible full dcKO_CAG-Cre mutants: (A, C, E, G, I, K) E13.5 dcKO_CAG-Cre mutant embryos were treated with TAM at E9.5, and whole-embryo sections were immunostained with antibodies against BAF155 (A), BAF170 (C), Brg1 (E), $\mathrm{Brm}(\mathrm{G}), \mathrm{BAF} 47$ (I), and BAF250a (K). (B, D, F, H, J, L) Quantifications of fluorescent signal intensities obtained from the sections described ( $A, C, E, G, I, G)$ (see also Table $S 1$ for statistical analysis). The results revealed that the protein expression levels of BAF155 and BAF170 were reduced throughout the TAM-treated dcKO_CAG-Cre mutant embryos, confirming the double knockdown of BAF155/BAF170. The expression levels of the other tested BAF subunits were also diminished in mutant embryos compared to controls. Scale bar $=500 \mu \mathrm{m}$.

In different tissues and cell lineages, BAF155 is highly expressed in proliferating stem/progenitor cells but generally down-regulated upon differentiation (Yan et al., 2008; Ho et al., 2009a; Tuoc et al., 2013). Conversely, little BAF170 is expressed in stem/progenitor cells (e.g., embryonic stem cells, or ESCs) and at higher levels in differentiated cells (e.g., neurons) (Yan et al., 2008; Ho et al., 2009a; Tuoc et al., 2013). We hypothesized that although only low expression levels are detected for BAF170 in proliferating ESCs and for BAF155 in post-mitotic neurons, this expression 
is necessary and sufficient to stabilize the embryonic stem cell (es)BAF and neuronal (n)BAF complexes. Indeed, when we derived ESC lines from blastocysts and primary neurons from forebrains (both representing the dcKO_CAG-Cre genotype), we found that the depletion of BAF155 and BAF170 in these cultured cells led to the loss of BAF subunit expression at the protein level (Narayanan et al., 2015).

These results collectively indicate that the knockout of BAF155/BAF170 in dcKO mutants eliminates the presence of known BAF complex subunits both in vitro and in vivo. Thus, the $d c K O$ mutants provide a potent tool for investigating the roles of entire BAF complexes during development.

\subsubsection{The loss of BAF complexes induces the accumulation of H3K27me2/3- marked heterochromatin}

Previous studies suggested that the loss of individual BAF subunits has a local (not global) influence on chromatin marks (Ho et al., 2011; Tuoc et al., 2013). However, when we examined epigenetic marks in cortex-specific dcKO_Emx1-Cre mice, which lacked entire BAF complexes, we observed a global reduction in euchromatin along with increased H3K27me2/3 and decreased H3K9Ac in the developing cortex during both embryonic and perinatal stages, as assessed by assays such as ChIP-Seq, immunohistochemistry, and western blotting (Narayanan et al., 2015). Thus, our data showed for the first time that the presence of BAF complexes is needed to maintain the balance between global repression and local activation of epigenetic programs during cortical development (Narayanan et al., 2015). 
The intriguing observation that BAF complexes are lost from the telencephalon-specific dcKO_FoxG1-Cre and inducible full dcKO_CAG-Cre mutants prompted us to study how this BAF155/BAF170 loss-of-function affects the H3K27me3 repressive mark. We performed western blotting (WB) on telencephalic tissue lysates from E11.5 dcKO_FoxG1-Cre mutants using an antibody against H3K27me3. Similar to our observation in cortical tissues, we found that the loss of BAF155 and BAF170 increased the level of H3K27me3 in telencephalon (Fig. 2.4A/C). Likewise, compared to control (non-injected) embryos, the H3K27me3 level was augmented in E13.5 dcKO_CAG-Cre embryos that had been injected with TAM at E9.5 (Fig. 2.4B/C). H3K27me2 and -3 are chromatin modifications that have been linked to the downregulation of gene expression (Cao et al., 2002; Pereira et al., 2010). Thus, the massive enhancement of H3K27me3 in the dcKO mutants would be expected to trigger obvious repression of gene expression. Indeed, gene expression profiling of developing cortices from $d c K O$ mutants revealed that most of the transcripts were down-regulated, with only a few showing up-regulation (Narayanan et al., 2015). Remarkably, BAF complexes were found to positively regulate most of the genes that are repressed by the H3K27 methyltransferase, Ezh2 (Pereira et al., 2010; Narayanan et al., 2015). 
A

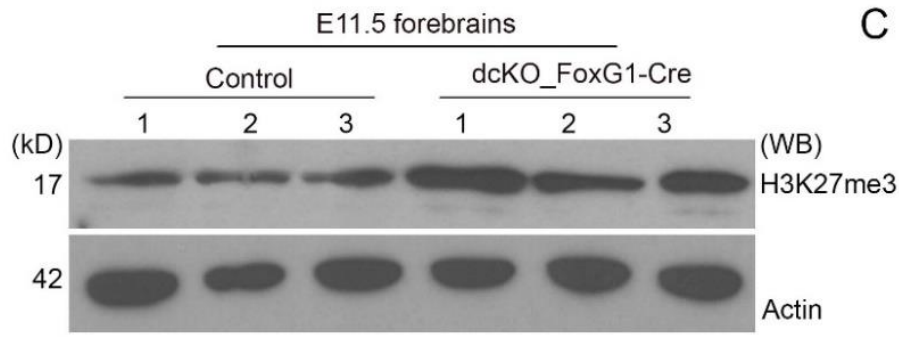

B

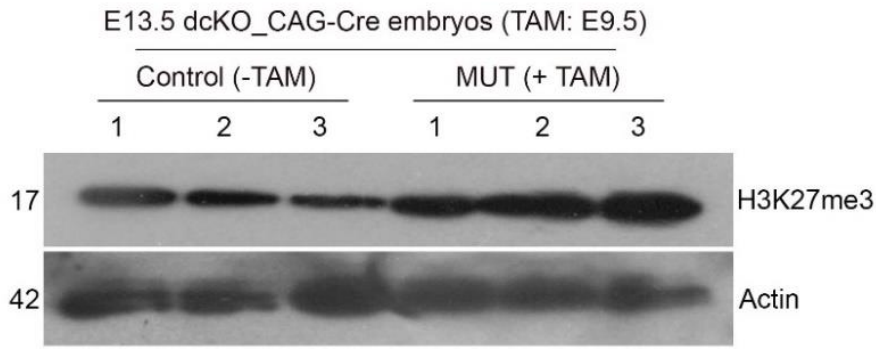

D

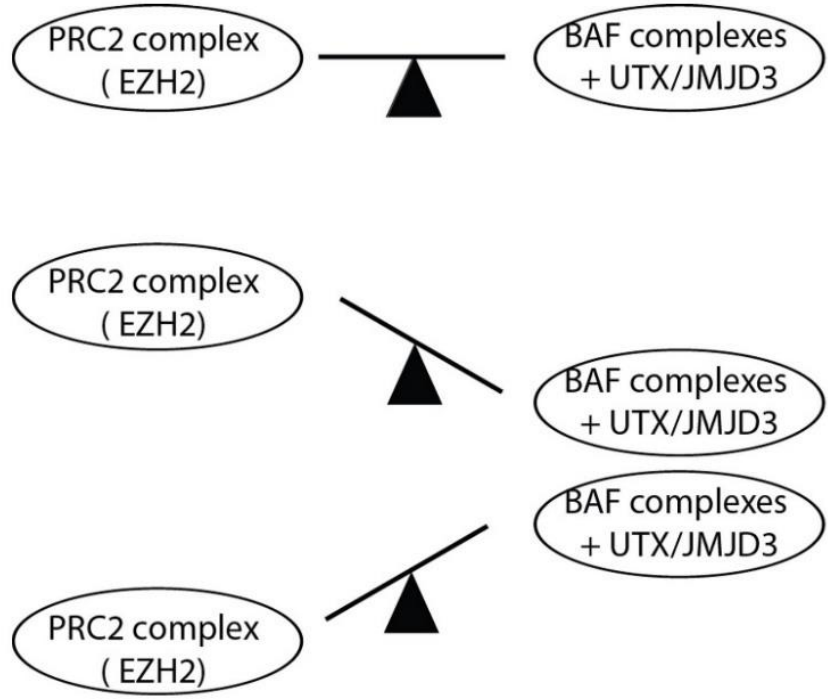

C

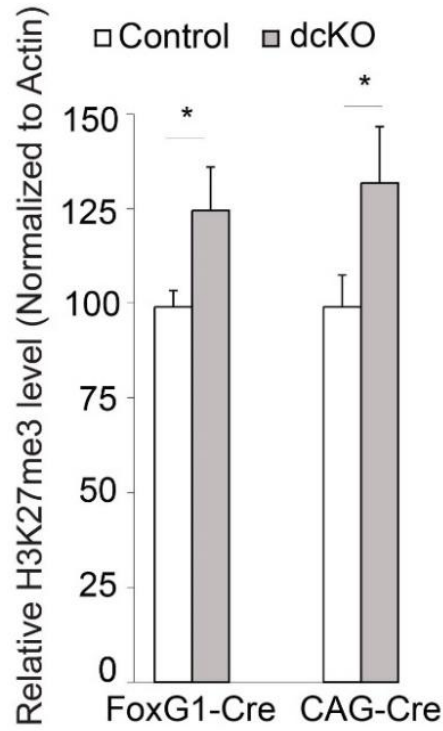

Normal condition

Increased H3K27Me2/3,

$\rightarrow$ Impairment of development, Tumorigenesis

Decreased H3K27Me2/3, Impairment of development

Figure 2.4 BAF complexes control the level of H3K27me3 in the brain and whole embryo during development: (A) WB analysis of E11.5 telencephalons from telencephalon-specific dcKO_FoxG1-Cre mutants revealed that the lost expressions of BAF155 and BAF170 elevated the level of ${ }^{\mathrm{H}} 3 \mathrm{~K} 27 \mathrm{me} 3$. (B) dcKO_CAG-Cre embryos treated with TAM at E9.5 showed up-regulation of H3K27me3 at E13.5, compared to untreated control embryos. (C) Densitometric quantification of the WB bands shown in (A and B; see also Table S2 for statistical analysis). (D) Schematic indicating how altered levels of H3K27me2/3 demethylases (UTX/Kdm6a and JMJD3/Kdm6b), BAF complexes, and the H3K27 methyltransferase Ezh2 subunit of the PRC2 complex collectively modulate histone methylation, developmental defects and diseases (e.g., tumorigenesis).

To directly examine the apparent opposing activity of BAF complexes and the Ezh2 subunit of the PRC2 complex, we treated dcKO mutants with an Ezh2 inhibitor and examined gene expression in developing cortex. We found that 
inhibition of the H3K27 methyltransferase partially rescued the expression of certain BAF-complex target genes (Narayanan et al., 2015). In mechanistic terms, our results suggested that this process involves binding of the BAF155 and BAF170 core subunits of the BAF complex to the JmjC domains of UTX/Kdm6a and JMJD3/Kdm6b, which are required for the $\mathrm{H} 3 \mathrm{~K} 27 \mathrm{me} 2 / 3$ demethylase activities of these proteins (Narayanan et al., 2015).

Ezh2 (or PRC2) (Cao et al., 2002; Shen et al., 2008; Pereira et al., 2010) and UTX/Kdm6a/JMJD3/Kdm6b (Agger et al., 2007; De Santa et al., 2007; Hong et al., 2007; Jepsen et al., 2007; Lan et al., 2007; Lee et al., 2007; Xiang et al., 2007) are the only enzymes known to methylate and demethylate H3K27, respectively. These enzymes play essential roles in development and diseases by modulating gene expression programs through changes in the methylation of H3K27. Studies have shown that homozygous-null Ezh2 mutants die prior to completing gastrulation, conditional loss of maternal Ezh2 results in severe growth retardation among neonates (Erhardt et al., 2003; Puschendorf et al., 2008), and EZH2 overexpression causes tumorigenesis (Varambally et al., 2008; Takawa et al., 2011). Phenotypic analysis revealed that mouse embryos $d c K O$ for Utx and Jmjd3 (which encode the H3K27 demethylases) exhibit lethality at mid-gestation. Moreover, the expression levels of Jmjd3 and Utx are significantly decreased in several types of primary tumors (Agger et al., 2009). The BAF complexes, which we identified as important cofactors of the H3K27 demethylases, are known to be key players in development (Ko et al., 2008; Ho \& Crabtree, 2011; Narayanan \& Tuoc, 2014; Narayanan et al., 2015) and tumor suppression (Ko et al., 2008; Wu, 2012; Helming et al., 2014; MasliahPlanchon et al., 2015). Thus, any alteration in the balance among the BAF complexes, H3K27 demethylases, and methyltransferase will result in severe developmental 
defects and/or diseases such as cancer (Fig. 2.4C; (Shi, 2007; Pedersen \& Helin, 2010; Ho \& Crabtree, 2011; Kadoch et al., 2016)).

\subsection{Conclusion}

We herein present evidence suggesting that BAF155 and BAF170 act as scaffolding subunits to maintain the stability of BAF complexes. The loss of BAF complexes in BAF155/BAF170 double mutants is associated with severe defects in global epigenetic and gene expression programs during cortical development (Narayanan et al., 2015) and embryogenesis (this study). Our results further suggest that manipulation of the endogenous expression and activity levels of the chromatin-remodeling BAF complexes, the H3K27me2/3 demethylases (UTX/Kdm6a and JMJD3/Kdm6b), and the H3K27me2/3 methyltransferase (polycomb repressive complex 2) might enable to alter global gene expression programs. The crosstalk between BAF complexes and epigenetic factors revealed herein may shed light on how cells acquire their fates. This work could thus contribute to the establishment of protocols aimed at differentiating specific lineages from pluripotent cells and/or treating diseases.

\subsection{Materials and Methods}

\subsubsection{Transgenic mice}

Floxed BAF155 (Choi et al., 2012), floxed BAF170 (Tuoc et al., 2013), FoxG1-Cre (Hebert \& McConnell, 2000) and CAG-Cre (Hayashi \& McMahon, 2002) mouse lines were kept in a C57BL6/J background. All animal research was conducted in accordance with the local regulations for animal protection. 


\subsubsection{Immunohistochemistry (IHC) and Western blotting (WB)}

IHC and WB were performed as previously described (Tuoc \& Stoykova, 2008; Tuoc et al., 2009). The following polyclonal (pAb) and monoclonal (mAb) primary antibodies used in this study were obtained from the indicated commercial sources: Brg1 rabbit pAb (Santa Cruz), Brg1 mouse mAb (Santa Cruz), Brm mouse mAb (BD Biosciences), Brm rabbit pAb (Abcam), BAF250 mouse mAb (Sigma), BAF170 rabbit pAb (Bethyl), BAF170 rabbit pAb (Sigma), BAF155 rabbit pAb (Santa Cruz), BAF155 mouse mAb (Santa Cruz), BAF60a mouse mAb (BD Biosciences), GAPDH rabbit pAb (Santa Cruz), B-actin rabbit pAb (Sigma), and H3K27me3 rabbit pAb (Upstate). The utilized secondary antibodies included peroxidase-conjugated goat anti-rabbit $\lg$ ( $1: 10,000$; Covance), peroxidase-conjugated goat anti-mouse IgG (1:5000; Covance), and Alexa 488- or Alexa 568-conjugated IgG (various species,1:400; Molecular Probes).

\subsubsection{Imaging and quantitative and statistical analyses}

Imaging was performed with an Axio Imager M2 (Zeiss) with a Neurolucida system (Version 11; MBF Bioscience) and confocal fluorescence microscopes (TCS SP5; Leica). Pictures were analyzed further with Adobe Photoshop. Densitometric quantification of WB bands and quantitative analysis of IHC signal intensities were performed using the ImageJ software. Statistical analyses were carried out using the Student's t-test. The results are presented as the mean \pm SEM. 


\section{CHAPTER 3: BAF (mSWI/SNF) complex regulates mediolateral patterning in developing forebrain}

Huong Nguyen*, Godwin Sokpor*, Arpan Parichha, Linh Pham, Yuanbin Xie Joachim

Rosenbusch, Mehdi Pirouz, Rüdiger Behr, Anastassia Stoykova, Jochen F. Staiger, Shubha Tole, Tran Tuoc

${ }^{*}$ Authors with equal contribution.

This chapter is a prepared manuscript. Its submission for publication is forthcoming.

Personal contributions: I coequally contributed to performing most of the phenotype characterization. I also contributed to drafting, modification, and finalization of the manuscript.

\subsection{Abstract}

Early patterning determines the correct specification and formation of functional cortical areas, including appropriate generation and distribution of neuronal subtypes during brain development. Specific signaling and transcription factors (TFs) are known to tightly regulate patterning of the dorsal telencephalon (dTel) to afford proper cortical arealization and morphogenesis. Nevertheless, linking chromatin changes to transcriptional program(s) that control cortical patterning remains elusive. Here, we report that the BAF chromatin remodeling complex spatiotemporally regulates patterning of the mouse forebrain. Conditional ablation of BAF complex in the dTel neuroepithelium caused dysregulation of cortical patterning. Specifically, the cortical hem and medial cortex abnormally expanded beyond their boundaries leading to 
compromised mediolateral cortical partitioning. Consequently, the hippocampal primordium is not specified, and cortical identity is disturbed in the BAF complexdeficient dTel. We provide evidence that BAF complex modulates the mediolateral patterning possibly by interacting with the TF LHX2 and effecting the LHX2-dependent transcriptional activity. Comparative analysis of BAF complex and LHX2 expression in the mouse and marmoset embryonic cortex revealed their plausible evolutionary role in dTel patterning. Our results suggest a mechanistic synergy between BAF chromatin remodeling complex and LHX2 in regulating forebrain patterning and ontogeny.

\subsection{Introduction}

Telencephalic patterning is crucial for the anatomical and functional designation of various aspects of the brain during embryogenesis. Broadly, the dTel is organized into the cerebral cortex and the dorsal midline. The cerebral cortex is sub-divided into the neocortex and hippocampus, whereas the cortical hem and choroid plexus makeup the dorsal midline (reviewed in (Hébert \& Fishell, 2008)). Commitment of portions of the telencephalic neuroepithelium to these various structural and specialized functional domains takes place between E10.5 and E12.5 (Levitt et al., 1997; Gitton et al., 1999; Mallamaci et al., 2000). Activity of morphogens are known to orchestrate forebrain patterning through their gradient expression and ability to regulate other modulators, including transcription factors that play instrumental roles in the size and positional specification of telencephalic structures (Sur \& Rubenstein, 2005; Mallamaci \& Stoykova, 2006; Rash \& Grove, 2006; O'Leary \& Sahara, 2008). Specialized telencephalic structures, such as the cortical hem, rostral telencephalic organizer, and anti-hem are known to instruct patterning of the dTel (Reviewed in (Kiecker \& Lumsden, 2012)). 
The cortical hem is a telencephalic structure flanked by the hippocampal primordium and the choroid plexus. It is a prominent source of patterning signals, including those that belong to the Wingless/Int (WNT) (Grove et al., 1998) and the Bone morphogenetic protein (BMP) families (Furuta et al., 1997). Thus, the cortical hem performs important role(s) in cortical patterning due to its morphogenetic enrichment. Induction of the hippocampal fields in the medial telencephalic neuroepithelium, and the establishment of the presumptive cortex are modulated by the cortical hem during telencephalic patterning (Grove et al., 1998; Lee et al., 2000; Mangale et al., 2008a; Caronia-Brown et al., 2014). Additionally, the cortical hem is known to generate Reelin-producing Cajal-Retzius cells that migrate to populate the MZ of the cerebral cortex, and perform important role in cortical and hippocampal lamination (Rice \& Curran, 2001; Meyer et al., 2002; Takiguchi-Hayashi et al., 2004; Bielle, F. et al., 2005; Forster et al., 2006).

Proper molecular regulation and cytoachitecture are prerequisite for the establishment of the various cortical domains during dTel patterning (reviewed in (Subramanian \& Tole, 2009b)). A few factors such as Foxg1, Emx1/2, Pax6, Lhx2, Fgf, Lmx1a, Gli3 (Grove et al., 1998; Bishop et al., 2000b; Mallamaci et al., 2000; Bulchand et al., 2001; Shimogori et al., 2004; Shinozaki et al., 2004; Kimura et al., 2005; Muzio \& Mallamaci, 2005; Mangale et al., 2008a; Chizhikov et al., 2010; Godbole et al., 2018), and components of WNT, FGF and BMP signaling and associated mechanisms (Forster et al., 2006; Hébert \& Fishell, 2008), have been reported to be central in directing cortical patterning. Genetic manipulation of these factors cause various cortical abnormalities, including expansion or non-specification of the hem, fate switch of hem cellular derivatives, non-induction or dysgenesis of the hippocampus, and perturbation of neocortical patterning. Although the aforementioned factors are essential for forebrain patterning, the interconnecting mechanistic network involved is quite unclear. 
The Brg1/Brm-associated factor (BAF) or mammalian SWI/SNF complex is a multisubunit ATP-dependent chromatin remodeler. It is capable of re-structuring chromatin through histone mobilization and/or recruitment of epigenetic cofactors to modify the epigenetic landscape of chromatin leading to regulation of gene expression and related cell biological processes during brain development (reviewed in (Sokpor et al., 2017)). We previously developed mouse models of inactive BAF complex achieved by double conditional knockout (dcKO) of the scaffolding BAF subunits BAF155 and BAF170 in neural cells leads to disassembly of other subunits in the BAF complex and subsequent elimination by the ubiquitin-proteasome machinery (Narayanan et al., 2015; Bachmann et al., 2016; Nguyen et al., 2016). The aberration of the entire BAF complex function in dcKO or partly in single knockouts of BAF155 or BAF170 disturbs several aspects of neural development, including cortical and hippocampal histogenesis (Tuoc et al., 2013; Narayanan et al., 2015; Bachmann et al., 2016; Nguyen et al., 2016; Tuoc et al., 2017; Narayanan et al., 2018; Nguyen et al., 2018).

Interestingly, the BAF complex and transcription factor LHX2 appear to share common developmental functions. This is because, ablation of the BAF complex leads to neurodevelopmental phenotypes similar to that of Lhx2 deletion in several neural tissues, including the eye and olfactory neuroepithelia. Disturbance of Lhx2 expression during eye development causes mis-regulated retinal neural progenitor proliferation and differentiation, and gliogenesis (Lamba et al., 2008; Tétreault et al., 2009; Balasubramanian et al., 2014; de Melo et al., 2016; de Melo et al., 2018), a phenotype partly recapitulated by BAF complex manipulation in the developing human fetal retina (Lamba et al., 2008). Also, the olfactory epithelium of Lhx2 mutant mice have abnormal progenitor differentiation, deprivation of olfactory receptor gene expression, and loss 
of neuronal identity leading to perturbed development and diversification of olfactory receptor neurons (Hirota \& Mombaerts, 2004; Kolterud et al., 2004). Comparatively, mice with conditional inactivation of the BAF complex in the olfactory epithelium presented with neurodevelopmental disturbances, including loss of LHX2+ olfactory receptor neurons and overall dysgenesis of the olfactory epithelium (Bachmann et al., 2016). Notably, both BAF complex and LHX2 are known to regulate neurogenesis during forebrain development in a WNT-signaling dependent manner. As a result, the respective mutants displayed reduced cortical size due to decrease in cortical neurogenesis (Hsu et al., 2015; Nguyen et al., 2018). Altogether, these similarities in the neurodevelopmental phenotypes in the absence of optimal BAF complex and LHX2 functionality motivated us to investigate the role of BAF complex in cortical patterning, which role is definitely known for LHX2.

In this study, we identified the BAF complex as a determinant of cortical identity and suppressor of the cortical hem during patterning of the forebrain. Interestingly, we found that the BAF complex interacts with LHX2 and is required for normal LHX2 transcriptional activity in the dTel, suggesting their functional interaction in maintenance of the correct mediolateral patterning in developing forebrain. Indeed, the cortical phenotype consequent to Foxg1-Cre and Emx1-Cre-mediated ablation of BAF complex largely phenocopied the Lhx2 null dTel. Particularly, loss of BAF complex resulted in expansion of the cortical hem at the expense of the medial and dorsolateral cortical primordia leading to distortion of mediolateral cortical patterning. Additionally, we show the evolutionary significance of the differential expression of BAF complex subunits in mouse and the more evolved marmoset cortex and hem. These results suggest the potential morphogenetic role of the BAF complex in regulating the anatomical and functional regional organization of the mammalian cerebral cortex. 


\subsection{Results}

\subsubsection{Loss of BAF155 and BAF170 phenocopies cortical hem expansion as in Lhx2-/- forebrain}

We examined the telencephalic phenotype of the dcKO_Foxg1-Cre mutant brain in which the BAF complex is conditionally abolished by deleting the subunits BAF155 and BAF170 in the entire mouse forebrain (Bachmann et al., 2016) with Foxg1-Cre activity recognizable from E8.5 (Hebert \& McConnell, 2000). Following the very early Foxg1_Cre-mediated ablation of the BAF complex, it was observed that the Glast immuno-delimited cortical hem, which is normally restricted to the edge of the medial pallium, was markedly expanded beyond its developmental spatial confinement as compared with wild-type at E11.5 (Fig. 3.1A/B; white arrowed). As revealed by our quantitative analysis of the 3D-reconstructed Glast-stained hem, we noticed the E11.5 dcKO_Foxg1-Cre cortical hem had undergone about 3-fold volumetric enlargement (Movie S1). Thus, the dcKO_Foxg1-Cre cortical hem is seen to have extended beyond its usual medial location to lateral aspect of the dTel neuroepithelium which is essentially the neuroepithelial domain of the presumptive cortex (Fig. 3.1A). By applying antibody against the cortex selective marker, LHX2, it was evident that the size of LHX2-marked cortical primordium was drastically reduced in the dcKO_Foxg1Cre dorsal pallium as compared with control (Fig. 3.1A).

Given the overlap in neurodevelopmental function(s) between the BAF complex and LHX2, we set out to identify similarities in telencephalic phenotype between the dcKO_Foxg1-Cre and Lhx2-/- mouse mutant brain. Interestingly, the E12.5 Lhx2-/telencephalon riboprobed for Wnt2b (a selective marker of hem) and Dbx1 (a selective marker of antihem) displayed dramatic expansion of the cortical hem and antihem, 
respectively (Fig. 3.1C; (Bulchand et al., 2001; Mangale et al., 2008; Roy et al., 2014; Godbole et al., 2018)). This resulted in loss of cortical domain of the Lhx2 mutant dTel. However, since Lhx2 is mainly expressed in the neocortex, the Foxg1-marked ventral telencephalon is not affected by the Lhx2 mutagenesis (Fig. 3.1C) as compared with an obvious early ventral telencephalic dysgenesis following loss of BAF complex in the forebrain (Fig. 3.1A). This is because the BAF complex is ubiquitously expressed, that is, in both dorsal and ventral Tel (Fig. S3.1) and the Foxg1-Cre is active in the neuroepithelium in both regions (Hebert \& McConnell, 2000).

Although we did not find definite alteration of the antihem in the dcKO_Foxg1-Cre dTel as compared with that in the Lhx2-/- (Fig. 3.1B), we were fascinated by the similar outcome in terms of hem expansion and compromised cortical identity. This motivated us to determine if both BAFs and Lhx2 act along similar axis to regulate cortical development. 

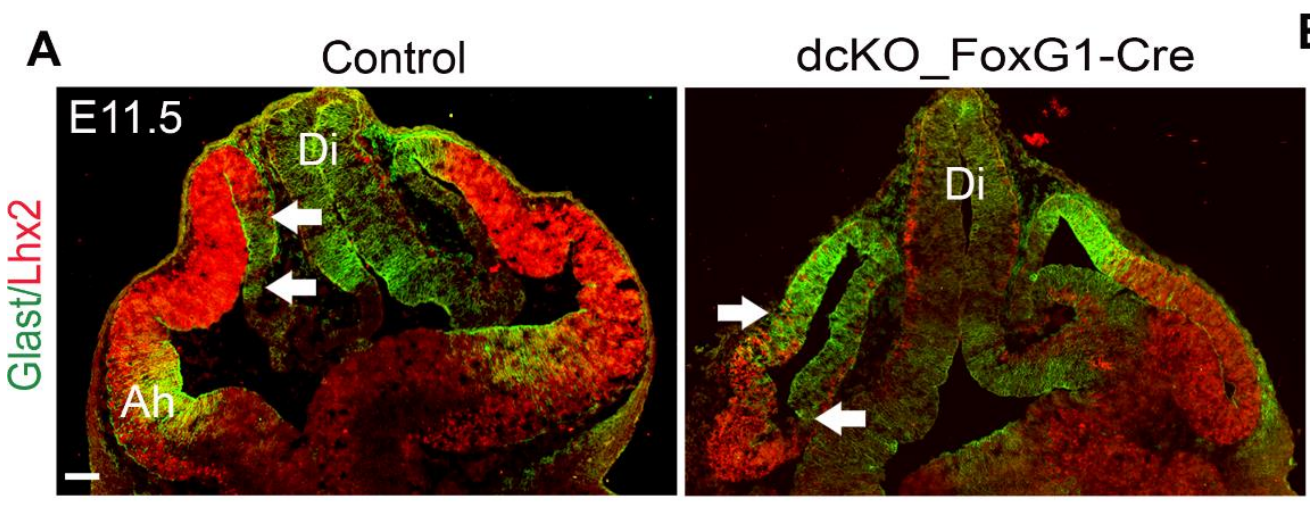

B $\square$ Control $\square$ dcKO_FoxG1-Cre

C

Lhx2-/- (E12.5)
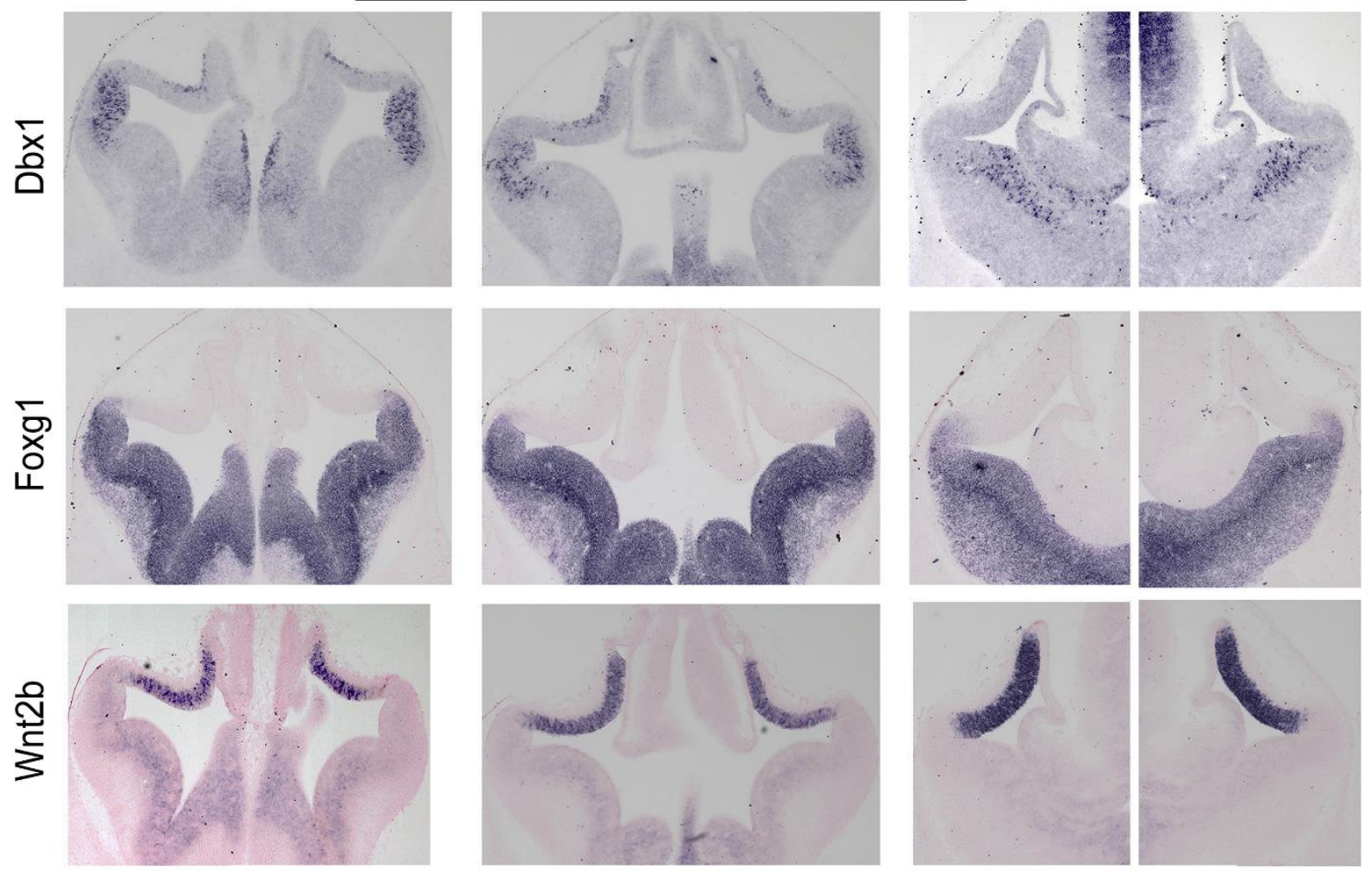

Rostral

Caudal

Figure 3.1 The cortical hem phenotype of the early developing BAF complex mutant forebrain resembles that of Lhx2 null mutant: (A) Images of the E11.5 mouse whole head sections showing the developing brain stained with LHX2 and Glast antibodies to reveal the presumptive neocortex (in vivid red) and the cortical hem (delineated with white arrows) of the control and dcKO_Foxg1-Cre telencephalon, respectively. The anti-hem (Ah) is not obvious in mutants as compared with control. (B) Bar chart indicating the statistical difference between the estimated total volume of the control and the dcKO_Foxg1-Cre cortical hems. (C) In situ hybridization images showing the rostral to caudal E12.5 whole mouse head (telencephalon) sections riboprobed for Dbx1, Foxg1, and Wnt2b which reveal the antihem, ventral telencephalon and cortical hem, respectively. The cortical hem is indicated by white arrows. Unpaired Student's $t$-test: ${ }^{* * *} \mathrm{P}<0.0005 ; \mathrm{n}=6$; Scale bar: $100 \mu \mathrm{m}$. Result are presented as mean \pm SD. Abbreviations: Ah, Anti-hem; Di, Diencephalon. 


\subsubsection{BAF155 and BAF170 interact with the transcription factor LHX2 during cortical formation}

We explored the molecular interaction partners of the BAF complex that control formation of the cortical hem and modulate hem-dependent establishment of other dTel structures. To this end, we performed co-immmunoprecipitation (colP) in cells lysates obtained from the E13.5 or E17.5 telencephalic tissue from wild-type mice, and culture NS-5 line neural stem cells (NSC) using antibodies against BAF155 and BAF170 followed by mass spectrometry (MS) (Narayanan et al., 2015; Nguyen et al., 2018). Many factors essential for neural morphogenesis were identified as protein interaction partners of both BAF155 and BAF170 subunits of the BAF complex. Strikingly, we found that the LIM homeodomain transcription factor LHX2, a determinant of cortical identity and suppressor of hem fate (Bulchand et al., 2001; Monuki et al., 2001; Mangale et al., 2008a; Roy et al., 2014), was bound to BAF155 and BAF170 in cultured NSCs, in the early (E13.5) and late (E17.5) developing forebrain (Fig. 3.2A/B).

Consistent with the findings in our protein-protein interaction investigation, we found extensive co-expression of BAF155 and BAF170 with that of LHX2 in the E11.5 presumptive wild-type cortex (Fig. S3.6). In the absence of the BAF complex, Lhx2 expression in the cortical neuroepithelium remained unaltered as compared with control (Fig. S3.2A/C; Fig. S3.6), indicating that the BAF complex does not have major influence on the expression level of Lhx2 in the presumptive cortex at early stage.

In other to establish a definitive functional interaction between BAF complex and LHX2, and its neurodevelopmental relevance in the context of hem morphogenesis and cortical formation, we investigated whether the interaction between BAF155/BAF170 
and Lhx2 influences LHX2-dependent transcriptional activity in the developing mouse cortex. For this purpose, we used an Lhx2-dependent reporter vector (pGL35xLhx2BS-luciferase), containing five Lhx2 binding sites upstream of a luciferase reporter and an empty vector without Lhx2 binding site (pGL3-luciferase) as a negative control (Folgueras et al., 2013). To eliminate the function of BAF55 and BAF170, the reporter plus Cre-expressing plasmids were co-electroporated into the E12.5 cortex of $B A F 155^{f / f f l} ; B A F 170^{f / / f l}$ embryos (Fig. 3.2C). We then examined isolated tissue samples from the cortex using a luciferase assay. Our analyses indicated that BAF complex knockout in the dTel (pallium) significantly diminished the reporter activity of pGL3$5 \times$ Lhx2BS-luciferase, but not that of the control plasmid which lack Lhx2 binding motif (Fig. 3.2C/D). This suggests that BAF155 and BAF170 are required for activation of Lhx2-dependent transcriptional activity.

Furthermore, we hypothesized that BAF complex might act together with LHX2 to regulate the expression of genes governing establishment of cortical identity or patterning. Pax6 is likely to be one of such genes, as it is a key regulator of cortical progenitor identity and patterning (Bishop et al., 2000; Stoykova et al., 2000; Toresson et al., 2000; Yun et al., 2001; Pinon et al., 2008). In cortical progenitors, LHX2 was also reported to directly regulate Pax6 expression by binding to its promoter (Hou et al., 2013; Shetty et al., 2013; Hsu et al., 2015). We first confirmed that Pax6 is downregulated in the dcKO dTel in our immunohistochemical (IHC) analyses (Fig. S3.2A/C). We then performed luciferase reporter assays to determine how the expression of Lhx2 and BAF complex regulate the activity of a Pax6 promoter vector, which contains Lhx2 binding sites (Kammandel et al., 1999; Hou et al., 2013). The Pax6 promoter vector driving luciferase expression (Pax6-luc) was transiently 
transfected into Neuro2A cells together with a combination of Lhx2-expression (CMVLhx2) and BAF155/BAF170-silencing vectors (Fig. 3.2E). After lysate collection and luminescence quantification, the results from the reporter assay revealed that as compared with control (in Pax6-Luc+CMV-EV condition), Lhx2 (in Luc+CMV-Lhx2 condition) significantly regulates promoter activity of Pax6 (Fig. 3.2E). However, upon BAF155/BAF170 dual knockdown using short-hairpin RNA (shBAFs) constructs, the level of luciferase reduced (Luc+CMV-Pax6+shBAFs condition), implying that both LHX2 and BAF155/BAF170 are required for maintaining the promoter activity of Pax6 (Fig. 3.2E).

Altogether, the results of our protein-protein interaction analyses and luciferase assay suggest that the forebrain patterning regulatory factor LHX2 plausibly acts in concert with BAF complex to orchestrate forebrain development. This finding inspired our interest in detailing the phenotype of the BAF complex mutant cortex in the direction of hem morphogenesis and related function in dorsal telencephalic patterning. 
A

\begin{tabular}{|l|l|r|r|r|r|r|r|}
\hline Protein & Accession & \multicolumn{4}{|c|}{ Number of peptides } \\
\hline & \multicolumn{3}{|c|}{ BAF155 colP/MS } & \multicolumn{3}{|c|}{ BAF170 colP/MS } \\
\hline & NSC & $\begin{array}{c}\text { E13.5 } \\
\text { forebrain }\end{array}$ & $\begin{array}{c}\text { E17.5 } \\
\text { forebrain }\end{array}$ & NSC & $\begin{array}{c}\text { E13.5 } \\
\text { forebrain }\end{array}$ & $\begin{array}{c}\text { E17.5 } \\
\text { forebrain }\end{array}$ \\
\hline & & 12 & 16 & 5 & 6 & 25 \\
\hline
\end{tabular}

B IP (with DNase, E12.5 cortex)

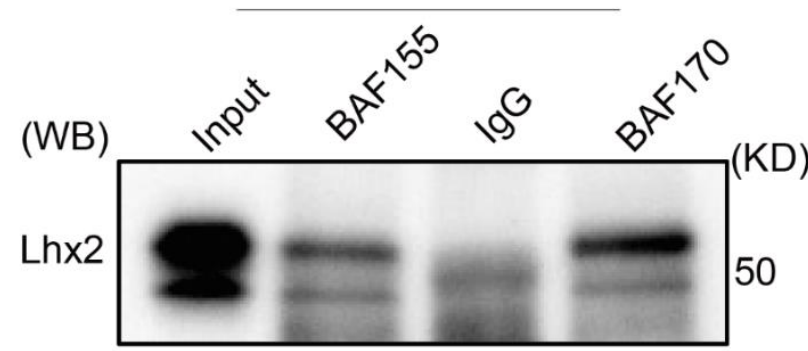

C

$\mathrm{E} 12.5$

(Baf155fl/fl, Baf170fl/fl)
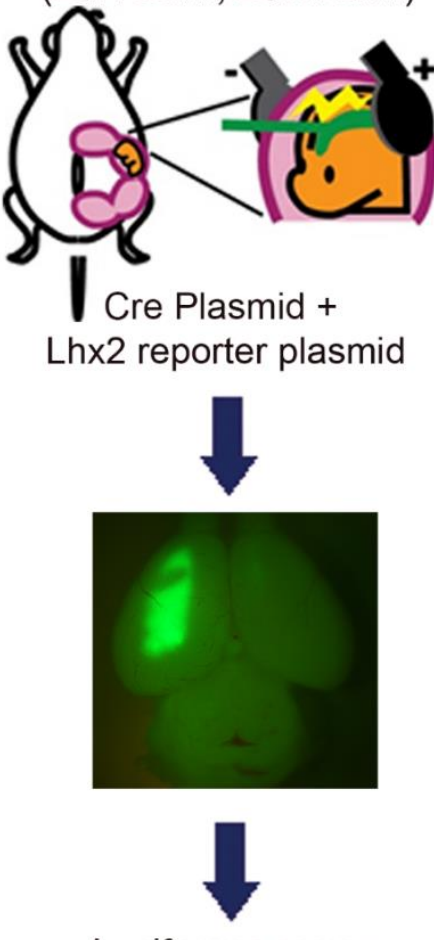

Luciferase assay

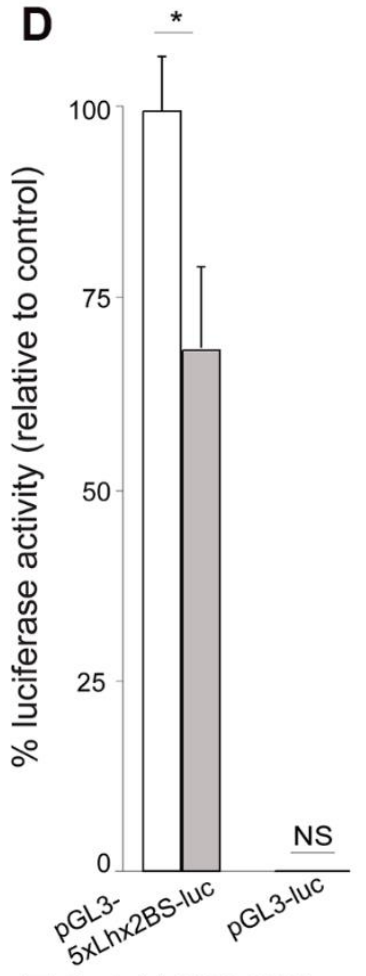

Control (pCIG2-GFP)

Figure 3.2 BAF complex cooperates with LHX2 to direct cortical development: (A) Image of table indicating the number of LHX2 peptides purified from immunoprecipitates of BAF155 and BAF170 obtained from NS5 cell protein extracts, and from the E13.5 or E17.5 dTel protein extract. (B) Interaction of BAF155 and BAF170 with LHX2 was confirmed by colP/western blot analyses of E12.5 dTel tissue. (C) Image showing scheme for in vivo luciferase assay. (D, E) Bar charts representing the statistical comparisons of (D) In vivo Lhx2-mediated luciferase activity in the control or dcKO_Emx1-Cre cortex, and (E) In vitro Pax6-mediated luciferase activity in culture Neuro2A cells in the presence or absence of BAF155, BAF170 or Lhx2 permutationally. Unpaired Student's $t$-test: ${ }^{*} \mathrm{P}<0.05,{ }^{*} \mathrm{P}<0.005 ; \mathrm{n}=6$; Result are presented as mean \pm SD. 


\subsubsection{BAF complex suppresses expansion of cortical hem at early corticogenesis}

To elucidate BAF complex function during formation of the dTel, we crossed mice harboring floxed alleles of BAF155 (Choi et al., 2012) and BAF170 (Tuoc et al., 2013)

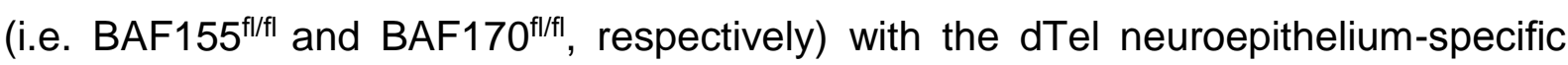
Emx1-Cre to generate dcKO_Exm1-Cre (Narayanan et al., 2015), in which Cre activity is detectable in the presumptive cortex from E10.5 onwards (Gorski et al., 2002). Thus, unlike in the dcKO_Foxg1-Cre telencephalon, in which BAF complex is broadly deleted at the initiation stage (E8.5) of telencephalic formation (Xuan, S et al., 1995; Martynoga et al., 2005; Bachmann et al., 2016), the Emx1-Cre practically restricted the loss of BAF complex to the dTel of dcKO_Emx1-Cre brain at the onset of neurogenesis (Fig. S3.1A/B; (Gorski et al. 2002)). Indeed, IHC analysis with antibodies against BAF155, BAF170 and other BAF complex subunits in the E11.5 and E13.5 cortices revealed loss of the BAF complex subunit expression in the entire cortex and dorsal part of the cortical hem by E11.5, whereas their expression in the ventral aspect of the cortical hem, the choroid plexus and ventral telencephalon are preserved in the dcKO_Emx1Cre mutants brain (Fig. S3.1; (Narayanan et al., 2015)).

We proceeded with characterizing the dTel phenotype of the dcKO_Emx1-Cre forebrain by initially reexamining our previously performed gene expression profiling of the dcKO_Emx1-Cre dTel containing both cortex and cortical hem at embryonic stage E12.5 (Fig. 3.3A). This analysis revealed that 1,723 transcripts were downregulated and 102 upregulated in the E12.5 dcKO_Emx1-Cre dTel (Narayanan et al., 2015). Notably, many genes important for cortical identity (e.g., Pax6, Ngn1, Ngn2, Tbr1 and Satb2) were downregulated, whereas expression levels of most known cortical hemspecific genes including Wnt3a, Wnt5a and Bmp were upregulated (Fig. 3.3A). 
We validated our RNA-seq data by examining the expression pattern and level of Wnt3a, which is mainly confined to the cortical hem at early stages of cortical development (Fig. 3.3B; (Lee et al., 2000; Louvi et al., 2007)). From our in situ hybridization (ISH) analysis, we found that the Wnt3a expression level and area of expression were increased in the mutant dTel as compared with control at E12.5 (Fig. 3.3B/E), thus corroborating the upregulated expression of Wnt3a seen in our RNA-seq data.

Besides measuring the expression pattern of upregulated genes, we also examined expression of the radial glial markers Glast and Blbp (Fig. 3.3C/D; Fig. S3.2A/C; Fig. S3.3A). Early in cortical development, between E11.5-E13.5, Glast expression displays a lateral ${ }^{\text {high }}$ to medial ${ }^{\text {low }}$ gradient with the highest level found in the cortical hem (Fig. 3.3C/D, demarcated with arrows). Analysis of the 3D-reconstructed E12.5 and E13.5 dcKO_Emx1-Cre cortical hem, as applied in dcKO_Foxg1-Cre hem analysis, revealed nearly 2-fold expansion by volume as compared with control (Fig. 3.3C/D; white arrows). Such expanded dcKO_Emx1-Cre hem was observed at rostrocaudal levels of the cortical hem system (Fig. 3.3C/F). Thus, our histological analyses suggest that the increased expression of hem marker genes in the RNA-seq data is likely due to cortical hem enlargement in the dcKO_Emx1-Cre forebrain at E12.5. That notwithstanding, the expanded cortical hem and associated dysmorphic cortical phenotypes are less severe in dcKO_Emx1-Cre in comparison to the dcKO_Foxg1-Cre dTel (Fig. 3.3D/G vs Fig. 3.1A/B). This is consistent with the report of Godbole et al. (2018), in which progressive later deletion of Lhx2 resulted in a diminishing expansion of the hem. 
We extended our analysis by looking at the cortical hem formation in dcKO_Emx1-Cre mutant from E11.5 (when the complete loss of BAF155 and BAF170 was detected earliest) until E14.5 (when the hypotrophic mutant cortex was adequately recognizable). Examination of the hem markers Glast and Blbp indicated that the cortical hem expansion had already commenced by E11.5 in the mutant cortex (Fig. S3.2A/B). Such mutant hem continued to abnormally expand at later (E13.5 and E14.5) cortical development stages (Fig. S3.2C/D; Fig. 3.5B/C).

To rule out the possibility that the BAF complex mutant hem progenitors/cells had adopted a hem-cortex double fate, we checked the expression of Pax6 and Lhx2, which are specifically expressed in the presumptive cortex (Fig. 3.1A; Fig. S3.2A/C). In both control and mutant, the expression of Pax6 and Lhx2 is excluded from the hem and limited to the cortical anlagen, indicating sufficient specification of the cortex and hem in the dcKO_Emx1-Cre telencephalon. Moreover, the E13.5 or E15.5 dcKO_Emx1-Cre cortex is capable of generating Tbr1+ and Ctip2+ post-mitotic neurons (Data not shown), hence confirming that the Lhx2 marked portion of the dcKO_Emx1-Cre dTel is indeed developing (neo)cortical tissue.

Of note, Emx1_Cre-mediated single knockout of either BAF155 (BAF155cKO) or BAF170 (BAF170cKO) did not elicit an overt expansion of cortical hem (Fig. S3.3) as seen in the dcKO_Emx1-Cre mutants. This implies that the dual function of BAF155 and BAF170 is necessary to orchestrate normal development (suppression) of the cortical hem system.

To determine how the BAF complex regulates hem cellular dynamics in the hem to suppress its expansion, we adopted the well-established experimental paradigm used 
to quantitatively assess neural progenitor cell cycle kinetics (Chenn \& Walsh, 2002; Tuoc et al., 2009; Tuoc et al., 2013; Bachmann et al., 2016; Nguyen et al., 2018). For this, E13.5 control and dcKO_Emx1-Cre cycling cortical hem neural progenitor/stem cells were pulse-labeled in vivo with the thymidine analog CldU for 24 hours, making it possible to mark recently exited (differentiated) progenitors in the cell cycle. Proliferating progenitors were marked with Ki67 immunostaining in the background of CldU immunoreactivity in the cortical hem which is typically outlined by fascicles of Glast+ fibers (Fig. S3.4A).

In comparison to the control cortical hem, it was observed that the BAF complex mutant hem was hypercellular and demonstrably enlarged with more cells positive for Ki67 antibody (Fig. S3.4A; green-labeled cells). However, cells positive for CldU but negative for Ki67 (CldU+/Ki67-) were considerably less in the mutant cortical hem as compared with control (Fig. S3.4A/B). The $\mathrm{CldU}_{+} / \mathrm{Ki67-}$ cortical hem cells are considered as those that have exited the cell cycle and have acquired differentiative fate. Statistical quantitative assessments of the cell cycle exit index of neural progenitors in the dcKO_Emx1-Cre cortical hem was thus significantly less than that in the control hem (unpaired $t$-test, $p$-value $=0.02 ; \mathrm{n}=4$ ).

In addition, we performed immunostaining for cleaved Caspase 3, a marker for apoptosis, to examine differences in programed cell death in the expanded dcKO_Emx1-Cre cortical hem as compared with that in the normal-sized control cortical hem (Fig. S3.4C). We observed more apoptotic cell death in the mutant cortical hem and the adjacent cortical primordium with Casp3 antibody staining compare with control (Fig. S3.4C). Significant number of the Casp3+ cells were also immuno-positive for Reelin in the E13.5 dcKO_Emx1-Cre dTel compare with control, thus confirming 
their Cajal-Retzius cell identity and hem origin (Fig. S3.4C; (Bielle, F. et al., 2005)). The identified Cajal-Retzius cells appear to have accumulated in the area of the prospective hippocampal anlage in the dcKO_Emx1-Cre dTel (Fig. S3.4C), and may be indicative of their impaired migration.

The requirement of BAF complex in temporal regulation of cortical hem size during corticogenesis was suggested by the outcome of the experiment in which the BAF complex was ablated later in cortical development in dcKO_hGFAP-Cre mutants in which Cre is late-active-around E13.5 (Zhuo et al., 2001; Nguyen et al., 2018). Notably, we observed no significant change in the size of the E15.5 cortical hem, (intensely stained with Glast antibody, and without Pax6 labeling) in dcKO_hGFAPCre dTel as compared with control (Fig. S3C; unpaired $t$-test, $p$-value $=0.926 ; n=4$ ). It implies that the modulatory function of the BAF complex on hem morphogenesis is exerted within a limited developmental time window. The temporal regulation of hem size was also reported in previous studies in which hem size was unaffected after later deletion of factors known to regulate hem size at early cortical development stages (Muzio \& Mallamaci, 2005; Hanashima et al., 2007; Mangale et al., 2008; Godbole et al., 2018).

Taken together, our RNA-seq and elaborate histological analyses indicate that the loss of BAF complex in the dTel neuroepithelium at the onset of cortical neurogenesis leads to aberrant expansion of the cortical hem partly as result of dysregulated hem progenitor cell kinetics and/or specification. This suggests that the BAF complex is a suppressor of hem fate during early forebrain development. 

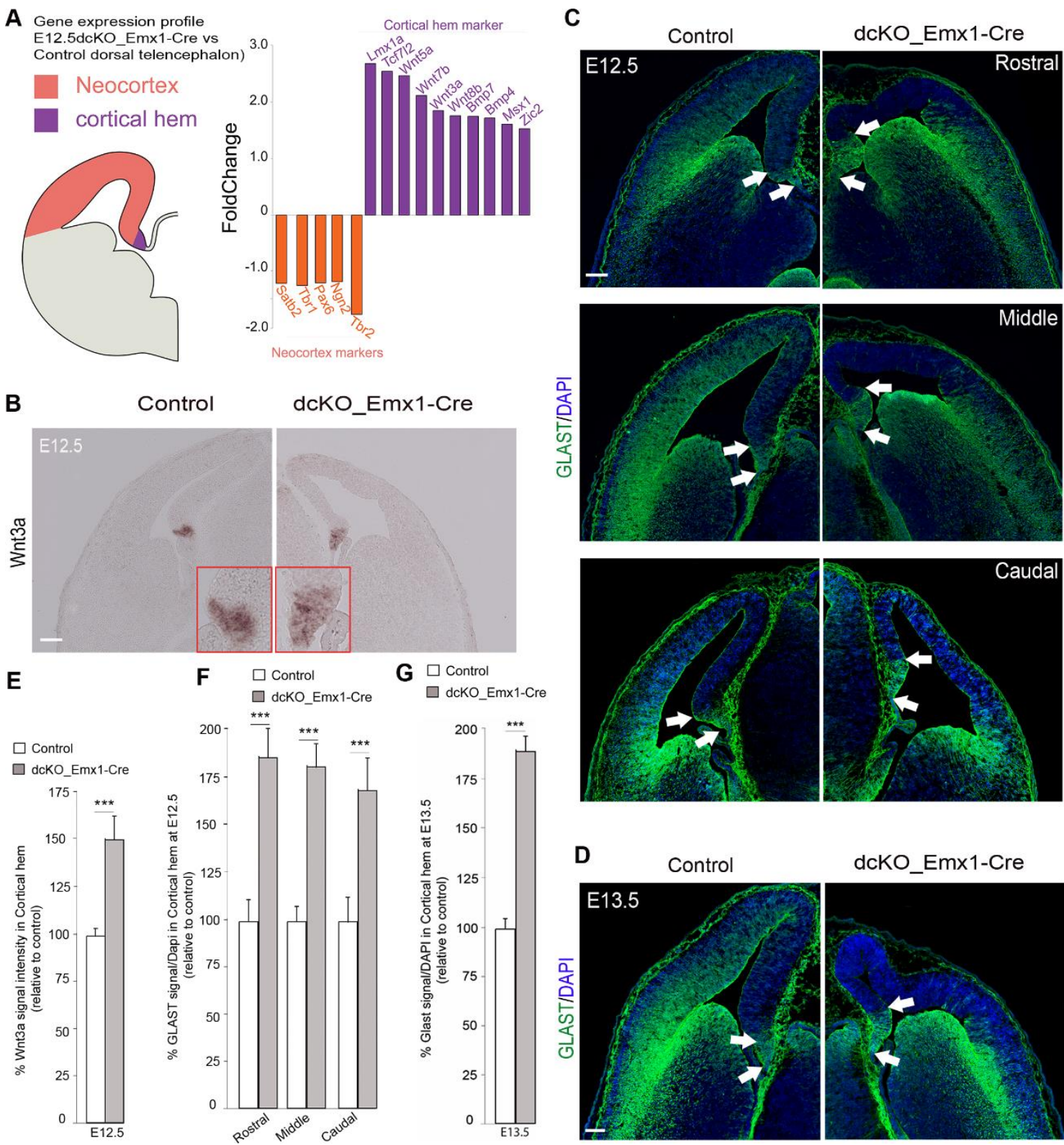

Figure 3.3 Increased expression of cortical hem-specific markers in dcKO_Emx1-Cre pallium: (A) Schematic showing demarcation of the neocortex (orange) from the cortical hem (purple) in hemisection of the mouse telencephalon. Results of RNA sequencing of the E12.5 control and dcKO_Emx1-Cre dTel tissues indicating fold change of downregulated neocortical marker genes (orange bars) and upregulated hem maker genes (purple bars) in the dcKO_Emx1-Cre are graphically represented. (B) Micrographs showing in situ hybridization in the E12.5 control and dcKO_Emx1-Cre dTel with the RNA probe generated from the hem marker Wnt3a. Inserts are zoom-ins of the control and mutant cortical hems revealed by the Wnt3a staining. (C, D) Images showing rostral to caudal sections of the E12.5 (C) and mid-section of the E13.5 (D) control and dcKO_Emx1-Cre brains immmunostained with the Glast antibody. White arrows point to the full length of the cortical hem revealed by intense Glast staining. (E-G) Bar graphs indicating the statistical difference between the estimated size of the control and mutant hem in the respective micrographs with: Wnt3a signal $(E)$, and Glast staining at E12.5 [rostral-caudal] (F) and E13.5 (G). Where shown, sections are counterstained with DAPI (blue). Unpaired Student's $t$-test: ${ }^{* \star *} P<0.0005 ; n=6$; Scale bars $=100 \mu \mathrm{m}$ in (B and D), $50 \mu \mathrm{m}$ in $(C)$. Result are presented as mean \pm SD. dTel: Dorsal Telencephalon. 


\subsubsection{Medial pallium is expanded and shifted laterally in the dcKO_Emx1-Cre mutant cortex}

Since loss of BAF complex in the cortical neuroepithelium resulted in abnormal expansion of the adjoining cortical hem, we turned our attention to possible impact on mediolateral patterning in the developing forebrain. We applied $\mathrm{ISH}$ analysis to examine expression pattern of medial Tel markers on serial coronal sections of E13.5 control and dcKO_Emx1-Cre forebrain (Fig. 3.4; Fig. S3.5).

Upon close examination, we observed abnormal patterning of the dorsal midline and neocortex in the dcKO_Emx1-Cre mouse dTel. Per Wnt2a and Wnt3a ISH, we noticed mild expansion of the ventral aspect of the dcKO_Emx1-Cre cortical hem as compared with control (Fig. 3.4B/C). However, the choroid plexus, which is the ventral continuum and derivative of the cortical hem (Louvi et al., 2007) did not present with any noticeable morphological alteration in dcKO_Emx1-Cre compared to control as indicated by ISH staining with the choroid plexus-specific marker transthyretin (TTR) (Fig. 3.4A; (Herbert et al., 1986; Grove et al., 1998; Monuki et al., 2001)).

Furthermore, ISH in rostrocaudal coronal sections of the E13.5 control and dcKO_Emx1-Cre head (telencephalon) with the RNA probe Lhx9 and immunostaining for Lef1 which partly reveal medial cortex/pallium identity, indicated drastic lateral expansion/extension of the medial cortex at the expense of the dorsolateral (neo)cortex in the dcKO_Emx1-Cre telencephalon (Fig. 3.4D/E). Normally, by E12.5, Lhx9 is strongly expressed in the hippocampal primordium and the expression intensity tapers from medial to lateral cortex. Around E13.5, Lhx9 expression is maintained in the ventricular zone of the hippocampal primordium and in postmitotic cells in the wild- 
type medial cortex (Fig. 3.4E; (Bulchand et al., 2003)). However, Lhx9 expression is observed to fill the whole of the medial cortex and strongly expressed in the lateral cortex of the dcKO_Emx1-Cre dTel (Fig. 4E). This deviation in Lhx9 expression from the aforementioned normal pattern consolidates the observed abnormal patterning in the dcKO_Emx1-Cre dTel. It means that the expanded cortical hem and lateralization of the medial cortex resulted in prepotency of the medial pallium in the entire dTel with progressive caudal prominence (Fig. 3.4E). The dramatically expanded hem in dcKO_Emx1-Cre (Fig. 3.4A-E) may have profound patterning implications, including elimination of the other dTel domains such as the neocortical maps that typify the properly patterned wild-type dTel.

Put together, these results highlight the regulatory function of the BAF chromatin remodeling complex in directing cortical patterning during mouse forebrain development. 


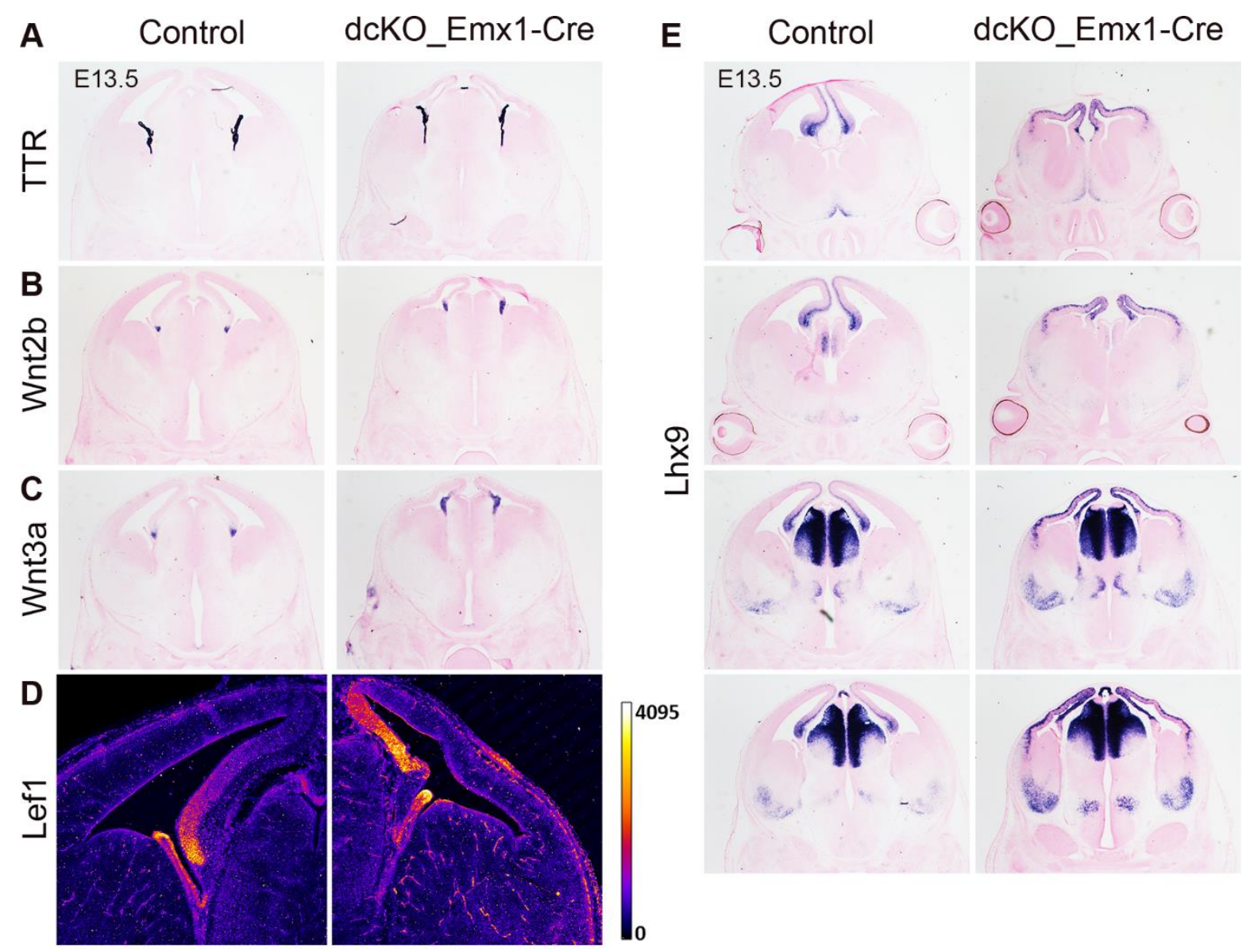

Figure 3.4 Early loss of BAF complex in the developing dTel distorts cortical patterning: Images showing in situ hybridization in the control and dcKO_Emx1-Cre E13.5 mouse (dorsal) telencephalon with the riboprobes TTR (transthyretin) to reveal the choroid plexus (A), Wnt2b and Wnt3a for the ventral cortical hem (B, C), Lef1 for the medial cortex (D), and Lhx9 for the cortical hem and medial cortex in rostral to caudal sections (E).

\subsubsection{BAF complex is required for the acquisition of cortical and hippocampal} identity

Molecular cues from the cortical hem, especially WNT signaling, have been shown to instruct proper establishment of the adjoining hippocampal fields (Galceran et al., 2000; Lee et al., 2000; Zhou et al., 2004; Machon et al., 2007). Moreover, it has been shown that ectopic hippocampal fields can be induced by ectopic cortical hems (Mangale et al., 2008). These provide definitive evidence that the hem is an organizing center indispensable in the specification and development of the hippocampal primordium. We previously reported that BAF155 and BAF170 are required for the 
development of the mouse hippocampus (Nguyen et al., 2018). These findings prompted us to investigate the implication(s) of the expanded cortical hem, as observed in this study, on the specification and morphogenesis of the hippocampal anlagen.

First, we examined the part of the dorsomedial telencephalic neuroepithelium that abuts the cortical hem for evidence of the hippocampal complex. To do this, we performed immunostaining of the E13.5 mouse telencephalon with the antibody Prox1, a selective maker for cells of the dentate gyrus. Normally, the dentate gyrus is formed at around E13.5 in the dorsomedial aspect of the telencephalic vesicles (Li \& Pleasure, 2005). However, there was no detectable Prox1 expression in the vicinity of the dorsomedial telencephalon atop the expanded BAF complex-ablated E13.5 cortical hem marked by Glast staining (Fig 3.5A).

Second, we performed immunostaining with the antibody Zbtb20, which characteristically reveals the identity of ammonic neuroepithelium-derived postmitotic neurons that will later form the Cornu Ammonis (hippocampus proper) (Xie et al., 2010; Nielsen et al., 2014; Nguyen et al., 2018). Previous studies in mice have shown that Zbtb20 is exclusively expressed in the hippocampal primordium between E12.5 and E13.5 and archicortical neurogenesis that leads to the generation of Zbtb20+ neurons commences by E14.5 in the mouse hippocampal fields (Fig. 3.5B; (Xie et al., 2010)). Surprisingly, there were no Zbtb20+ neurons in the location of the future hippocampal formation in the E14.5 medial pallium of dcKO_Emx1-Cre mutant compared with control (Fig. 3.5B). 
Using Pax6, AP2y, and Otx2 as indicators of cortical and hem identities, we observed that loss of BAF complex in dTel engendered massive shrinkage and loss of cortical integrity by mid embryonic corticogenesis (E14.5) in the dcKO_Emx1-Cre cortex as compared with control (Fig. 3.5C). The severely dysmorphic BAF mutant cortex is seen to have lost the medial to lateral gradient of Pax6 or AP2 $\gamma$ expression in the ventricular zone as normally found in the wild-type mouse cortex (Fig. 3.5C). Meanwhile, the Otx2 marked cortical hem remained expanded in the E14.5 dcKO_Emx1-Cre dTel as compared with control (Fig. 3.5C).

Interestingly, the non-specification of the hippocampus and loss of cortical structure in dcKO_Emx1-Cre forebrain phenocopies the Lhx2 null dTel (Chou et al., 2009; Godbole et al., 2018). Taken together, these findings indicate that the BAF complex is necessary for preservation of cortical identity and modulates correct formation of the cortical hem needed for hippocampal specification and morphogenesis. 

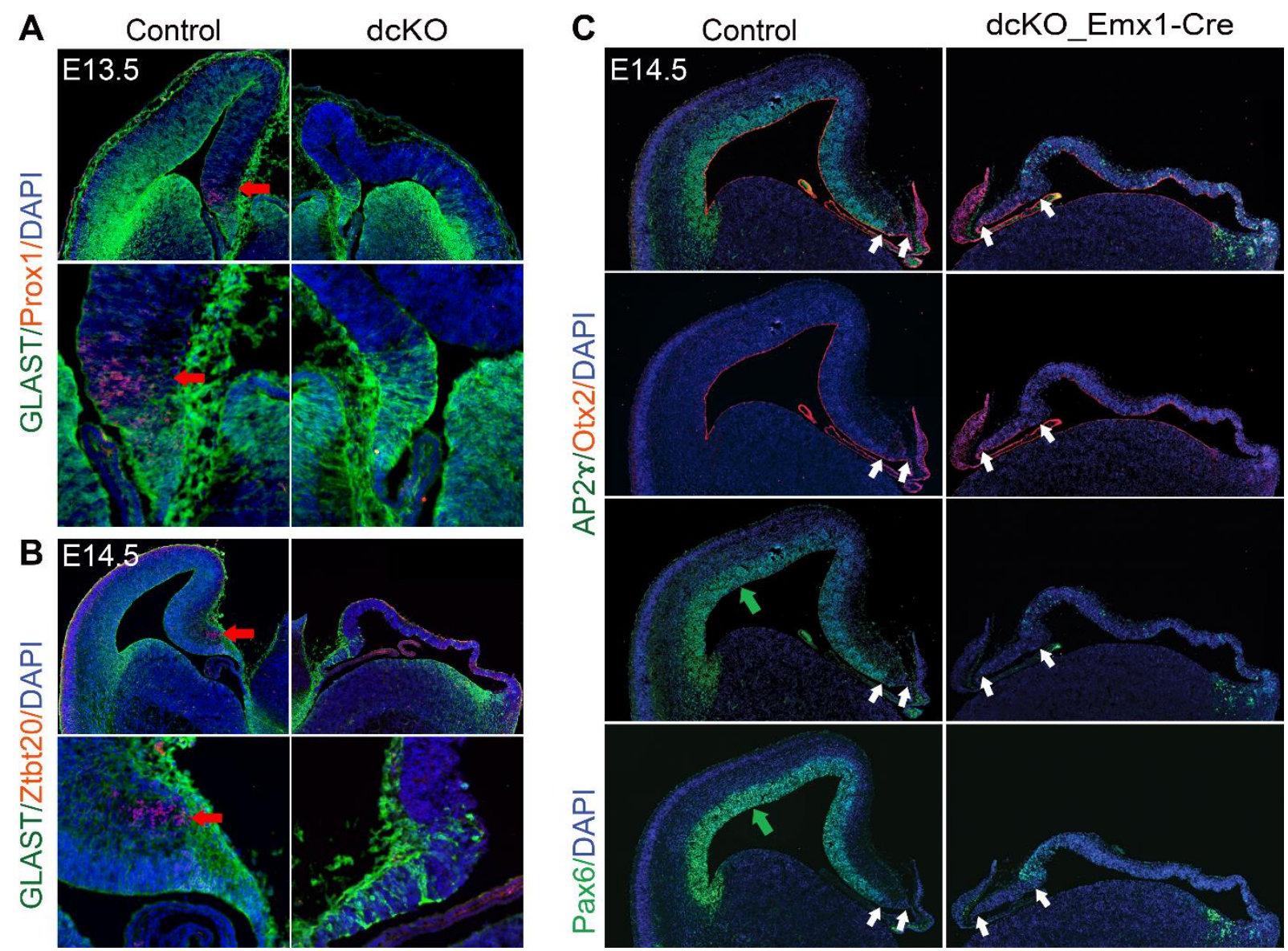

Figure 3.5 The BAF complex-ablated hem is incapable of hippocampal specification and neocortical patterning: (A) Immunohistochemical micrographs showing hemi-section of the E13.5 mouse brain stained with Glast and Prox1 antibodies in control and dcKO_Emx1-Cre. Panels of close up images highlight the presence and virtual absence of the Prox1+ dentate gyrus cells in the control and BAF complex mutant medial pallium, respectively. Red arrow points to Prox1 staining. (B) Micrographs showing hemi-section of the E14.5 mouse brain stained with Glast and Zbtb20 antibodies in control and dcKO_Emx1-Cre. Panels of close up images highlight the presence and absolute absence of the Zbtb20+ hippocampal neurons in the control and BAF complex mutant hippocampal primordium, respectively. Red arrow points to Ztbt20 staining. (C) Images showing profound loss of cortical mass and patterning associated with the expanded hem in the E14.5 dcKO_Emx1-Cre dTel as compared with control. The applied antibodies AP2y/Pax6, and Otx2 stain the cortical neuroepithelium/ventricular zone and cortical hem, respectively. White arrows indicate the size of the cortical hem. Sections are counterstained with DAPI (blue).

\subsubsection{BAF complex may not depend on WNT-signaling to modulate hem}

\section{formation and early cortical patterning}

To investigate the precise regulatory mechanisms by which BAF complex effects cortical hem suppression, we turned out attention to the involvement of the WNTsignaling pathway. Normally, several WNTs are distinctively expressed in the cortical hem early in telencephalic development, the earliest being Wnt3a (Fig. 3.3A/B; Fig. 
3.3C; (Grove et al., 1998)). From our RNA-seq data, we found indicators of increased WNT signaling in the E12.5 dcKO_Emx1-Cre dTel mainly in the form of increased fold changes (gene expression) of key WNT genes, including Wnt3a, Wnt5a, Wnt7b, Wnt8b, and the WNT signaling downstream effector TCF7L2 (Fig. 3.3A). Results of ISH with Wnt3a and Lef1 (another WNT signaling downstream effector) riboprobes in the E12.5 and E13.5 control and dcKO_Emx1-Cre telencephalon, respectively, reflected increased WNT signaling activity in/from the expanded mutant cortical hem (Fig. 3.4B/C/D). We previously demonstrated that downregulation of increased WNT signaling was able to rescue the malformed hippocampal phenotype in the BAF complex mutant (dcKO_hGFAP-Cre) brain (Nguyen et al., 2018). These results encouraged us to investigate whether the enlarged cortical hem structure seen in the dcKO_Emx1-Cre is as a result of increased mitogenicity caused by the WNT signaling hyperactivity.

To determine if the abnormally expanded cortical hem in BAF mutants is due to the plausible concomitant increase in WNT signaling, we adopt a chemical inhibition approach to downregulate the increased WNT signaling activity in the dcKO_Emx1Cre dTel (Nguyen et al., 2018). To achieve this, we performed scheduled intraperitoneal injection of pregnant mice within the active period of cortical hem development (i.e., E9.5-E12.5) with the chemical ICG001 (Fig. 3.6A). ICG001 is a small-molecule chemical substance known to effectively inhibit the WNT/ $\beta$-catenin cascade to extenuate related perturbations in vivo (Emami et al. 2004; Nguyen et al., 2018). The brains of ICG001-treated mutant (dcKO_Emx1-Cre) and control embryos were harvested at E13.5 and immunohistochemically examine for gross morphological changes in the cortical hem (Fig. 3.6A-C). As shown in Figure 3.5B/C, the abnormal expansion of the Glast+/Pax6- cortical hem and associated cortical abnormality were 
not attenuated by downregulating the elevated WNT signaling in dcKO_Emx1-Cre

forebrain. Thus, the suppressive function of the BAF complex in limiting cortical hem size to permit proper cortical identity acquisition is likely independent of WNT signaling activity.

A

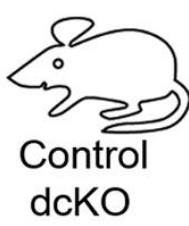

B Control

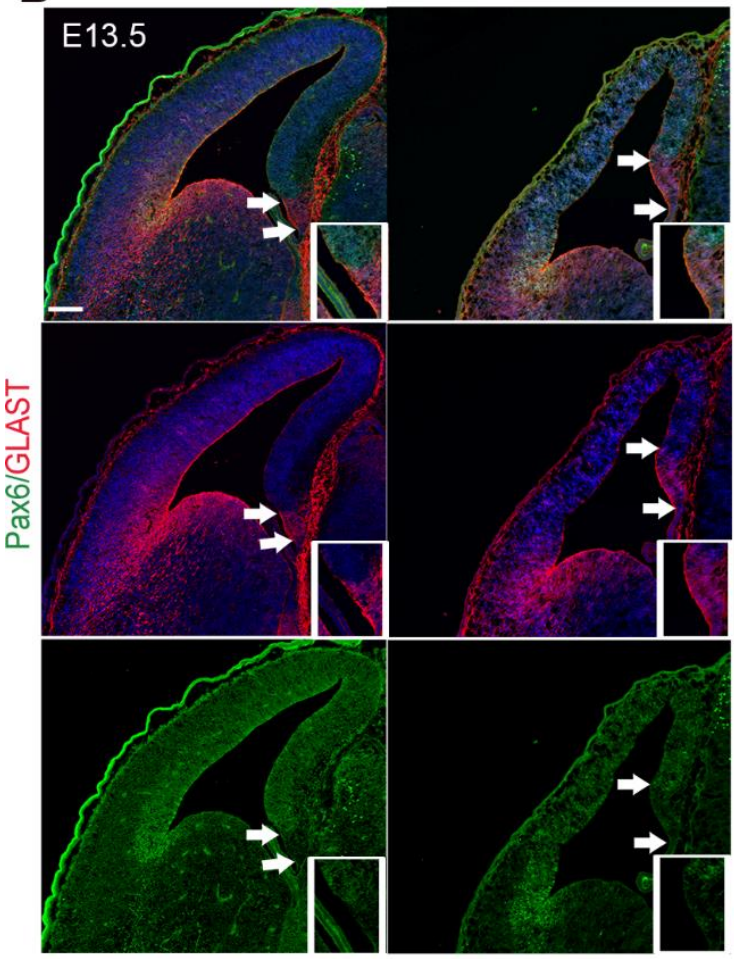

Wnt inhibitor (ICG001)

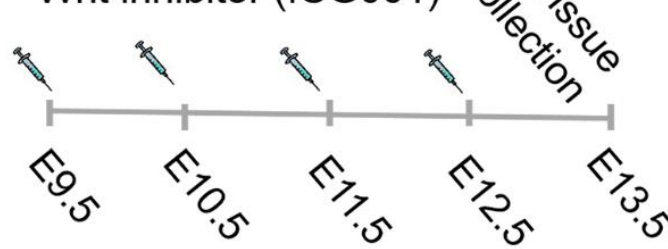

dcKO_Emx1-Cre dcKO_Emx1_ICG-001
C $\quad \square$ Control
$\square$ dcKO_Emx1-Cre
$\square$ dcKO_Emx1-Cre treated with Wnt inhibitor

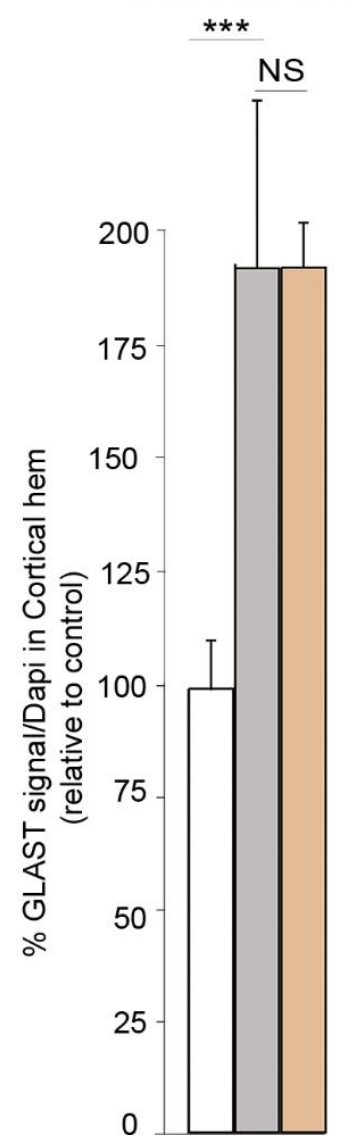

Figure 3.6 BAF complex acts independent of WNT signaling to suppress hem expansion: $(A)$ Illustration showing the experimental scheme employed to achieve in vivo chemical knockdown of the upregulated WNT signaling in the dcKO_Emx1-Cre telencephalon. Pregnant mice harboring both control and the BAF complex mutant embryos were intraperitoneally injection daily from E9.5 to E12.5 with the chemical WNT inhibitor ICG-001. The brains of ICG-001-treated embryos were harvested at E13.5 for immunohistochemical analysis. (B) Images of the E13.5 control (Wild-type) and dcKO_Emx1Cre WNT inhibitor-treated or -untreated brain sections stained with Glast and Pax6 antibodies. Inserts show close up view of the cortical hem. Sections were counterstained with DAPI (blue). The white arrows indicate the full extent of the cortical hem. (C) Bar chart representing the statistical comparison between the estimated control hem size and that of the dcKO_Emx1-Cre or dcKO_Emx1-Cre with WNT inhibitor treatment. Unpaired Student's $t$-test: ${ }^{* *} \mathrm{P}<0.0005$; NS: non-significant; $\mathrm{n}=6$; Scale bar: $=100 \mu \mathrm{m}$. Result are presented as mean \pm SD. 


\subsubsection{Expression pattern analysis of BAF155 and BAF170 in marmoset developing forebrain suggests their role in cortical hem formation and cortical patterning in primates}

Given that we identified the BAF complex to participate in cortical hem development via interaction with the key patterning factor LHX2 (this study) and Pax6 (Tuoc et al., 2013) in the developing mouse telencephalon, we sought to determine the implication of the morphogenetic function of the BAF complex during cortical structure evolution. For this purpose, we comparatively examined the expression pattern of BAF155 and BAF170 (BAF complex) in the early (E11.5-E13.5) developing mouse cortex (Fig. S3.1) and in the embryonic marmoset cortex at E74 (Fig. 3.7) as a common non-human primate model.

The expression pattern of BAFs in the developing mouse cortex is observed to be homogeneous and hence without any obvious graded expression (Fig. S3.1). Interestingly, however, we noticed discernable laterallow to medial ${ }^{\text {high }}$ gradient in the expression of BAF155 and BAF170 in the more evolved developing marmoset cortex at E74 (Fig. 3.7; Fig. 3.8). Likewise, we checked and compared the expression pattern of the known TFs Pax6 and LHX2 in the mouse and marmoset embryonic cortex. We noted that Pax6 and Lhx2 are similarly expressed in a gradient pattern in the marmoset and mouse cortex, although the LHX2 expression gradient (i.e., lateral ${ }^{\text {low }}$ to medial ${ }^{\text {high }}$ ) in the cortex of both species is in counter gradient to the Pax6 expression i.e., lateral ${ }^{\text {high }}$ to mediallow (Fig. 3.7; Fig. S3.2).

Graded expression of TFs is a key mechanism for patterning the telencephalon (Kimura et al., 2005). Strikingly, the graded expression of BAF complex in the E74 marmoset cortex is similar to that of LHX2 and is consistent with an evolutionary 
conservation of the interaction between both factors as identified in the mouse cortex (Fig. 3.2; Fig. S3.6; Fig. 3.7). The mouse hem expresses BAF complex genes, but this expression is lost in the marmoset (Fig. 3.7; Fig. 3.8). This may be an adaptive modification that subserves additional functions in the marmoset hem.

In a broader context, these observations may have evolutionary implication in primate cortical patterning and development, especially that the BAF complex has been identified to interact with, and augment the transcriptional activity of the well-known patterning factor LHX2 (Fig. 3.2; (Monuki et al., 2001; Mangale et al., 2008)).

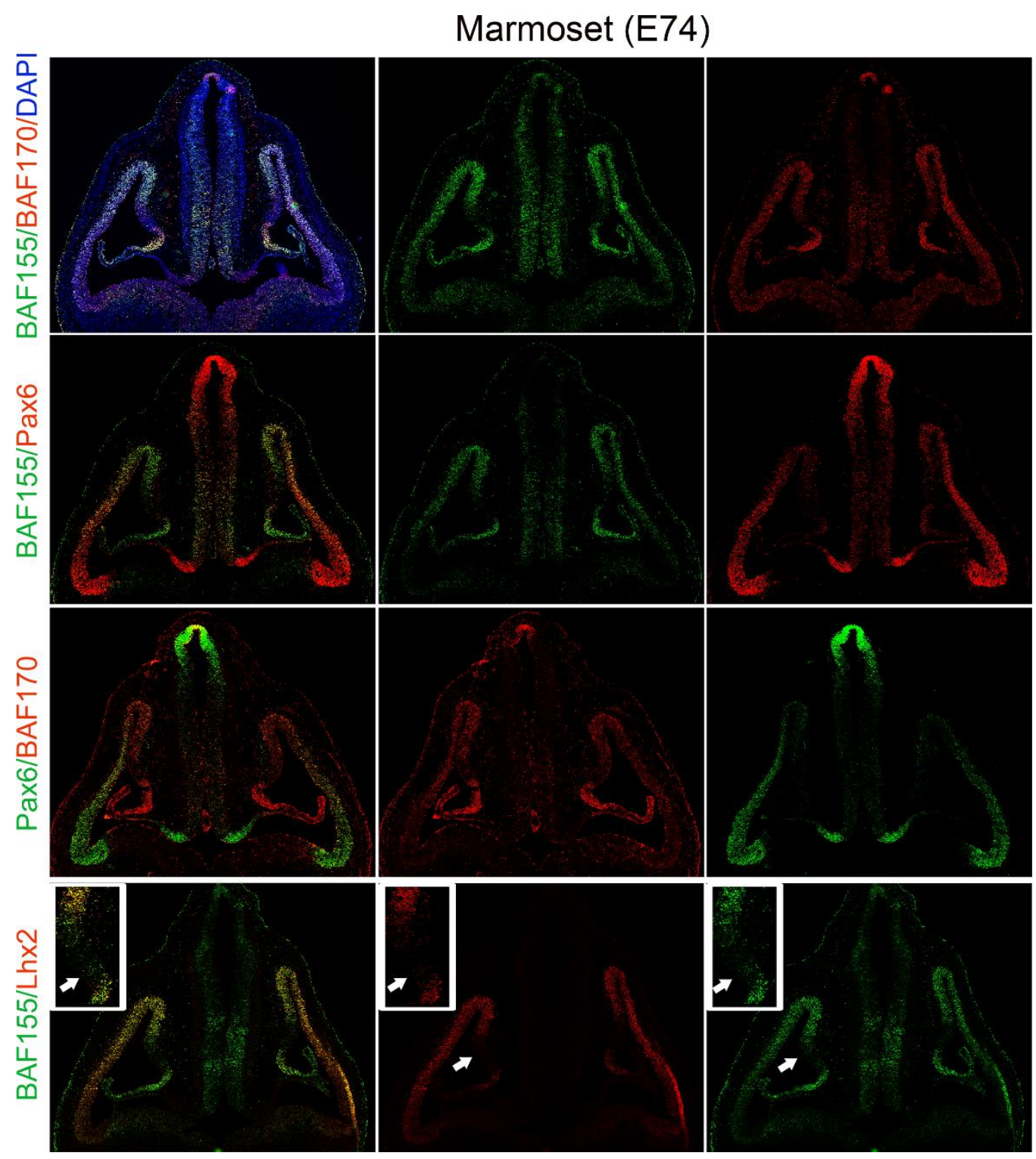


Figure 3.7 The morphogenetic function of BAF complex is evolutionarily refined: Images of the marmoset head showing immunostaining of the E74 telencephalon with BAF155, BAF170, LHX2, and Pax6 antibodies. In vivo molecular interaction of the BAF complex with the well characterized morphogenetic transcription factors LHX2 and Pax6 are shown by colocalization signals (yellow) in the respective labeled panels. Inserts are increased magnifications of the medial pallium indicating the exclusion of the BAF complex, LHX2 and Pax6 expression from the cortical hem domain.

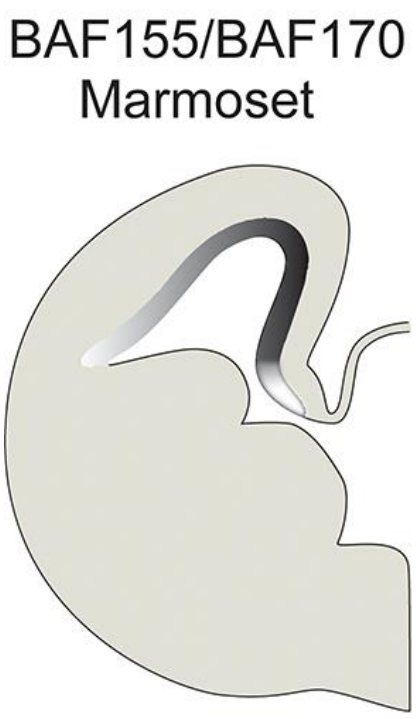

Neocortex
WT

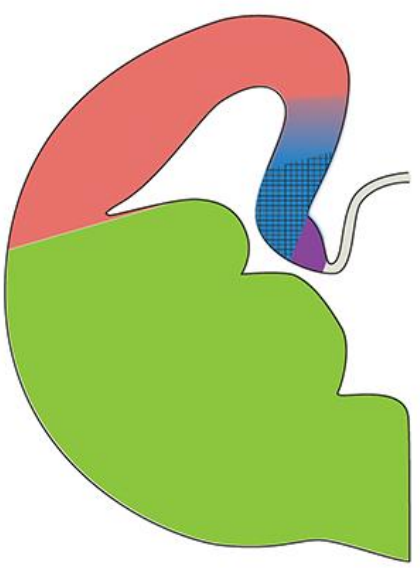

Medial palium
dcKO_Emx1-Cre

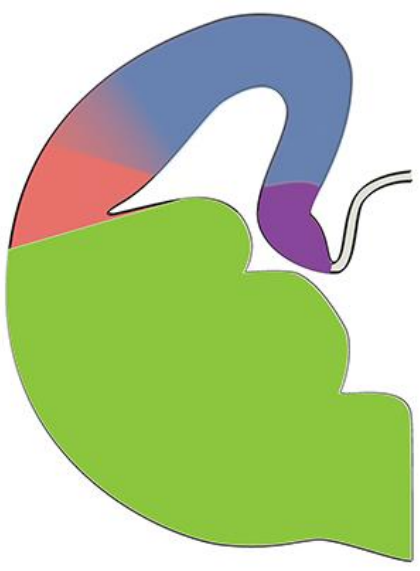

Cortical hem

\section{Hippocampal primordium}

\section{Ventral telencephalon}

Figure 3.8 Graphical illustration of normal and BAF155/170 knockout-induced dTel abnormal patterning: Illustrations showing gradient expression of BAF155 and BAF170 in the developing marmoset cortex, phenotype comparison between normal and abnormal cortical patterning in wild-type (WT) and dcKO_Emx1-Cre mouse dTel, respectively. The hem, hippocampus and neocortex are properly specified and placed in the wild-type dTel. However, the hem and medial cortex expand at the expense of the hippocampus and neocortex, making the dcKO_Emx1-Cre dTel have predominant medial cortex identity.

\subsection{Discussion}

\subsubsection{BAF complex orchestrates cortical patterning via regulation of cortical hem}

\section{formation during development of the telencephalon}

During development, the telencephalon is organized into structural domains that correlate with specific functional areas. The cortical hem and anti-hem are embryonic organizers located at the medial and lateral edge of the dTel, respectively. The cortical hem is a hub for morphogen signaling known to regulate areal patterning of the 
embryonic cortical neuroepithelium (reviewed in (Subramanian et al., 2009)). Absence or abnormal morphology of the cortical hem or molecular manipulations of related morphogenetic factors have been linked to various telencephalic perturbations of the developing forebrain, including cortical mis-patterning (Xuan, S et al., 1995; Lee et al., 2000; Martynoga et al., 2005; Yoshida et al., 2006; Mangale et al., 2008; Chizhikov et al., 2010; Caronia-Brown et al., 2014; Godbole et al., 2018). However, our understanding of correlating activity of chromatin regulators to the functionality of specific morphogens that drive forebrain patterning is far from clear.

In this study, we focused on determining the role of the ATP-dependent chromatin remodeler BAF complex in regulating forebrain patterning. Previous studies identified the BAF (mSWI/SNF) complex to play pivotal roles in many aspects of cortical development, including neural progenitor specification, proliferation and differentiation, and maturation of postmitotic neural cells (Reviewed in (Sokpor et al., 2017)). To expand our understanding of the molecular regulators that ensure the establishment and maintenance of cortical structures and function, we spatiotemporally inactivated the multimeric chromatin remodeling BAF complex via deletion of its scaffolding subunits BAF155 and BAF170 by using Foxg1-Cre, Emx1-Cre and hGFAP-Cre. By this means, we demonstrate in this current study that the BAF complex possesses morphogenetic capability that instructs patterning of the mammalian $\mathrm{dTel}$.

We observed that loss of BAF complex during early stages of cortical formation (i.e., under Foxg1- and Emx1-Cre drivers) caused abnormal expansion of the cortical hem and medial cortex to the detriment of the hippocampal complex and mediolateral cortical identity. The LHX2+ presumptive cortex, although specified in the BAF complex mutant background, appeared dysmorphic and lateralize. Cortical patterning was seen 
to be progressively lost from rostral to caudal levels of the telencephalon due to exaggeration of the medial cortical identity or lopsided medial cortical specification. This outcome points to possible distortion of the cortical neuroepithelial patterning due to BAF complex ablation, and highlights potential abolishment of the secondary organizer function of the expanded cortical hem. Such secondary patterning activity by hem may be necessary for tweaking neocortical arealization to yield the various functional cortical areas.

Given the important role of the cortical hem in dTel patterning, we partly think the BAF complex likely regulates formation of the cortical hem to modulate cortical patterning. This notion is consolidated by the observation that although the hem is expanded in the absence of optimal BAF complex function, the size of the choroid plexus, which is derived from the hem (Louvi et al., 2007) is not affected (Fig. 3.4A). It is also conceivable that the expanded hem is improperly configured, leading to its incompetence in orchestrating arealization of the adjacent cortical primordium or in executing other hem functions.

Indeed, the expanded hem in dcKO_Emx1-Cre forebrain was unable to specify the hippocampal anlagen. This is rather counterintuitive, given that deletion of BAF complex led to increase in WNT signaling in the hippocampus (Nguyen et al., 2018) and from the cortical hem (this study). Yet, the canonical WNT signaling is known to exerts mitogenic effect on neural progenitors leading to their enhanced proliferative capacity and subsequent generation of the hippocampal neurons (Lee et al., 2000; Chenn \& Walsh, 2002; Muzio \& Mallamaci, 2005; Pozniak \& Pleasure, 2006; Machon et al., 2007; Wrobel et al., 2007; Nguyen et al., 2018). As a result, WNT inhibition rescued abnormal phenotypes in the hippocampus (Nguyen et al., 2018), but 
surprisingly not the expanded hem in the BAF complex mutant forebrain (Fig. 3.6). Thus, although the BAF complex can regulate WNT signaling and its targets (Barker et al., 2001; Ronan et al., 2013; Vasileiou et al., 2015; Nguyen et al., 2018), we did not find evidence of its dependence on WNT signaling in modulating cortical hem morphogenesis.

Also, the expanded hem in the BAF complex mutant dTel is incapable of normal CajalRetzius cell generation due to their susceptibility to apoptosis. Cajal-Retzius cells are prominent derivatives of the cortical hem (Rice \& Curran, 2001; Takiguchi-Hayashi et al., 2004; Bielle, F. et al., 2005; Liu et al., 2018). They produce factors such as Reelin and p73 which are involved in cortical arealization and laminar patterning (Meyer et al., 2002; Meyer et al., 2004; Frotscher et al., 2009). It means that loss of BAF complex causes abnormal generation of Cajal-Retzius cells which partly underscores the observed mis-patterning seen in the mutant cortex. We reckon that it is possible the Cajal-Retzius cells produced by the expanded hem following BAF complex inactivation exhibit other deficits which need elucidation in further investigations.

Our findings convey the idea that the BAF complex likely suppresses inherent expansion tendencies of dTel midline structures, excluding the choroid plexus, during cortical patterning. However, the said suppressive capacity of the BAF complex is likely exerted at early cortical development stages, perhaps until E12.5, as loss of BAF complex from E13.5 achieved in dcKO_hGFAP-Cre mutants did not lead to an observable hem or medial cortex expansion (Fig. S3C), although hippocampal formation was perturbed in such mutants (Nguyen et al., 2018). The effect of BAF complex deletion on hem expansion is consistent with outcome of other studies in which genetic manipulation of factors such as Foxg1, Lhx2, Lhx2/Pax6 (double 
deletion), and Nf2 in early development caused abnormal expansion of the mouse dTel midline structures and associated cortical patterning deviations (Bulchand et al., 2001; Monuki et al., 2001; Vyas et al., 2003; Muzio \& Mallamaci, 2005; Mangale et al., 2008; Lavado et al., 2013; Godbole et al., 2017).

The morphogenetic function of the chromatin remodeling BAF complex in forebrain patterning is also brought into focus by the unique expression pattern displayed in the primate-like developing dTel (Fig. 3.8). Ordinarily, gradient expression of morphogens in the developing telencephalon is instructive for defining the various telencephalic domains, including the cortical hem (Reviewed in (Borello \& Pierani, 2010)). Conceptually, concentration of morphogens diffusely declines from their localized origin of production to confer placement identity on target cells. Such positional assignment also depends on a threshold of graded morphogens to regulate specific cell fate acquisition and thus contributing to tissue compartmentalization (Wolpert, 1986). Although we project the morphogenetic function of the BAF complex in mammalian forebrain, a gradient pattern of BAF complex subunits expression is not discernable in the mouse dTel. However, the more evolved marmoset dTel display such gradient that is in parallel or counter gradient with well-known telencephalic patterning factors like Pax6 and LHX2 (Fig. 3.7; (Borello \& Pierani, 2010)). Again, as opposed to the mouse hem, the marmoset cortical hem avoids BAF complex expression much as, at least, Pax6 and LHX2. This may be reflective of some kind of refinement in the expression of BAF complex and its morphogenetic function in the primate-like (marmoset) cortex as compared with the less evolved non-primate (mouse) cortex. 
These observations make us propose that the BAF complex may function as a direct or indirect epigenetic morphogen that have plausibly evolved in orchestrating the intricate patterning of primate cortex via harmonization of various cortical hemmediated patterning events that lead to the structural and functional regionalization of the mammalian dTel.

\subsubsection{Implication of BAF complex and LHX2 interaction in suppression of cortical hem expansion}

The formation of cortical hem is regulated by the activity of several factors, including the transcription factor LHX2 (Reviewed in (Subramanian \& Tole, 2009)). Previous studies reported cortical hem expansion with concomitant massive loss of the cortical identity due to complete or constitutive ablation of Lhx2 (Bulchand et al., 2001; Mangale et al., 2008). From our protein-protein interaction assay and mass spectrometry experiment, we found LHX2 as an interaction partner of the BAF complex. Since the cortical and hem phenotype caused by inactivation of BAF complex function in the dTel neuroepithelium (this study) resemble that of Lhx2 deletion (Mangale et al., 2008; Roy et al., 2014), it is likely that both factors have functional similarities in directing telencephalic development. We infer from our luciferase experiment that the identified interaction may be a potentiating kind to suppress hem expansion and preserve cortical identity. This is mainly because we found that the BAF complex promotes the Lhx2 gene target Pax6 which also participates in cortical patterning (Bishop et al., 2000; Stoykova et al., 2000; Kimura et al., 2005; Godbole et al., 2017). Moreover, either factor is unable to singularly afford sufficient suppression to prevent the abnormal expansion of the cortical hem in the absence of the other, hence signifying possible functional cooperation between the BAF complex and LHX2. 
Thus, we have made a novel finding in which we correlate transcriptional activity to specific epigenetic chromatin remodeling machinery that act in concert to provide proximal suppressive function in preventing the free enlargement of the cortical hem and associated cortical development perturbations.

Upon close analysis in comparing the Lhx2 and BAF complex-ablated hem phenotype, we suspected a temporal overlap in the mode of action of both factors in effecting hem size restriction. Whereas loss of BAF complex in the dTel under the Emx1 driver, with Cre activity beginning from E10.5 (Guo et al., 2000; Gorski et al., 2002), resulted in cortical hem expansion (this study), loss of Lhx2 at E10.5 or later did not have any impact on hem size compared to control (Godbole et al., 2018). Thus, it appears that the repressive function of the BAF complex on hem fate persists slightly beyond the apparent end point of the LHX2-dependent hem size restriction. To that end, we argue that the BAF complex is sufficient in limiting hem expansion, and may partly substitute for or complement the function of LHX2 in preventing the uncontrolled expansion of the cortical hem during later or active periods of hem morphogenesis.

In all, we suggest that the chromatin remodeling BAF complex, partly through interaction with LHX2, engages in the regulatory network that ensures the specification and morphogenesis of the mouse cortical hem, and its placement at the medial edge of the dTel. Our comparative analysis of the mouse and marmoset developing telencephalon makes it possible to extrapolate the relevance of the functional synchrony between BAF complex and LHX2 in orchestrating hem formation and cortical patterning in the primate forebrain. 


\subsection{Materials and methods}

\subsubsection{Animals}

Floxed BAF155 (Choi et al. 2012), floxed BAF170 (Tuoc et al., 2013), FoxG1-Cre (Hebert and McConnell 2000), Emx1-Cre (Gorski et al., 2002), and hGFAP-Cre mice (Zhuo et al., 2001) were maintained in a C57BL6/J background (license numbers 14/1636 and 16/2330).

Marmoset monkey (Callithrix jacchus) were obtained from the self-sustaining colony of the German Primate Center (Deutsches Primatenzentrum; DPZ) and housed according to the standard German Primate Center practice for common marmoset monkeys. Embryonic and fetal stages were obtained surgically by hysterotomy or hysterectomy (license numbers 42502-04-12/0708 and 42502-04-16/2129) as described previously (Aeckerle et al., 2015; Wolff et al., 2019). All surgical work on the monkeys was performed by a veterinarian with several years of experience in handling and operating marmoset monkeys. A detailed description of experiment with marmoset monkey is provided in Supplemental Information. Animals were handled in accordance to the German Animal Protection Law.

\subsubsection{Plasmids and antibodies}

A list of plasmids and antibodies with detailed descriptions is provided in Supplemental Information.

\subsubsection{In utero electroporation, and in vivo (Lhx2) and in vitro (Pax6) transcriptional activity assay}

In vivo and in vitro transcriptional activity assay were performed as previously described (Pinon et al., 2008; Nguyen et al., 2018). Briefly, Lhx2 transcriptional activity 


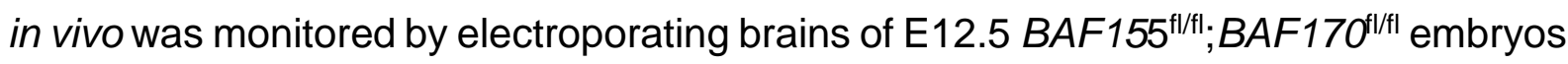
with a Cre plasmid (or empty plasmid as a control) and the reporter constructs pGL35xLhx2BS-luciferase or pGL3-luciferase (Folgueras et al., 2013) together with pRL-TK constructs at a 5:1:0.3 ratio. Lhx2 transcriptional activity was measured at E14.5. For in vitro assay, Neuro2A cells at $1 \times 10^{5}$ per well density were plated into 24 -well plates. Cells were transfected with $0.8 \mu \mathrm{g}$ of shRNA plasmids (ShBAF155, shBAF170) along with $50 \mathrm{ng}$ of Pax6-luc (Kammandel et al., 1999) and $10 \mathrm{ng}$ of pRL-TK. 2 days posttransfection, cells were collected for Pax6 transcriptional activity measurement. In all cases, firefly luciferase activity was normalized to that of Renilla luciferase.

\subsubsection{ColP/Mass spectrometry, RNA-seq}

Detailed descriptions were provided previously (Narayanan et al., 2015).

\subsubsection{In vivo pharmacological treatment}

Detailed descriptions for treatment of Wnt inhibitor (WNTi, ICG 001, XAV-939) were provided previously (Nguyen et al., 2018).

\subsubsection{IHC and Cell cycle index}

After blocking with $5 \%$ goat or donkey normal serum, sections were incubated overnight with primary antibody at $4^{\circ} \mathrm{C}$, and the signal was detected with a fluorescent secondary antibody (Alexa Fluor; 1:400; Invitrogen). Cell cycle exit was determined on coronal sections of E13.5 brains collected $24 \mathrm{~h}$ after CidU injection $(140 \mathrm{mg} / \mathrm{g})$ of pregnant mice at E12.5. After double IHC with CidU and Ki67 antibodies, the cell-cycle index was calculated as the number of $\mathrm{CidU}+/ \mathrm{Ki} 67$ - cells divided by total $\mathrm{CidU}+$ cells. 


\subsubsection{In situ hybridization (ISH)}

ISH was carried out as described previously (Tuoc et al., 2009).

\subsubsection{Reconstruction in 3D}

3D images of the cortical hem were constructed using Neurolucida software version 11.03. Consecutive sections (10 $\mu$ each) of BAF mutant and control brains were imaged in rostro-caudal order. Contours were drawn in each section based on the expression of hem-specific markers. The 3D reconstruction was produced from whole-stack contours. The contours were placed into sets for left and right hems. The volume estimation was done by using Neurolucida Explorer v. 11.03.

\subsubsection{Cell counts and quantitative analysis of IHC signal intensity}

Immunostaining in $\mathrm{IHC}$ images was quantified using anatomically matched forebrain sections. Nucleus-marker positive cells within the pallium of confocal images were counted for comparison. In most cases, cell counts of six matched sections were averaged from three biological replicates (control/dcKO pallium). In many cases, the number of lineage marker cells was quantified using total marker-positive cells alone, or by normalizing to the total number of DAPI+ (nucleus-stained) cells using the following equation: Normalized number $=$ marker-positive cell number/DAPI ${ }^{+}$cell number. Statistical analyses of histological data were performed using unpaired Student's $t$-test. All bar graphs are plotted as means \pm SD. All statistical tests are twotailed, and $p$-values are considered to be significant for $\alpha=0.05$. 


\subsubsection{Imaging, quantification, and statistical analyses}

All images were acquired with standard (Leica DM 6000) and confocal (Leica TCS SP5) fluorescence microscopes. Images were further analyzed with Adobe Photoshop. IHC and ISH signal intensities were quantified by using ImageJ software. Statistical analyses were done using Student's $t$-test. All graphs are plotted as mean \pm SD. All detailed statistical analyses for histological experiments are presented in Table S7.

\subsection{Acknowledgments}

We acknowledge T. Huttanus, H. Fett for their expert animal care and support. We also thank A. Messing, A. P. McMahon, A. Jones, F. Guillemot and E. Fuchs for providing reagents. This work was supported by the Universitätsmedizin Göttingen (T.T), TU432/1-1, TU432/1-3 DFG grants (T.T), Schram-Stiftung (T.T) and DFG-CNMPB (T.T, J.S).

\subsection{Author contributions}

H.N. and G.S. performed most of the characterization of dcKO phenotypes; A.P. and S.T. provided data in figures $1 \mathrm{C}, 4$ and S5, and S.T. contributed in revising the manuscript; M.P. and Y.X. performed the protein-protein interaction study; L.P. and J.R. contributed to histological analyses; R.B. provide marmoset tissue; J.F.S. provided research tools, transgenic lines, and contributed to discussions; T.T. conceived, and supervised the study; The authors declare no competing financial interests. 


\subsection{Supplemental figures}

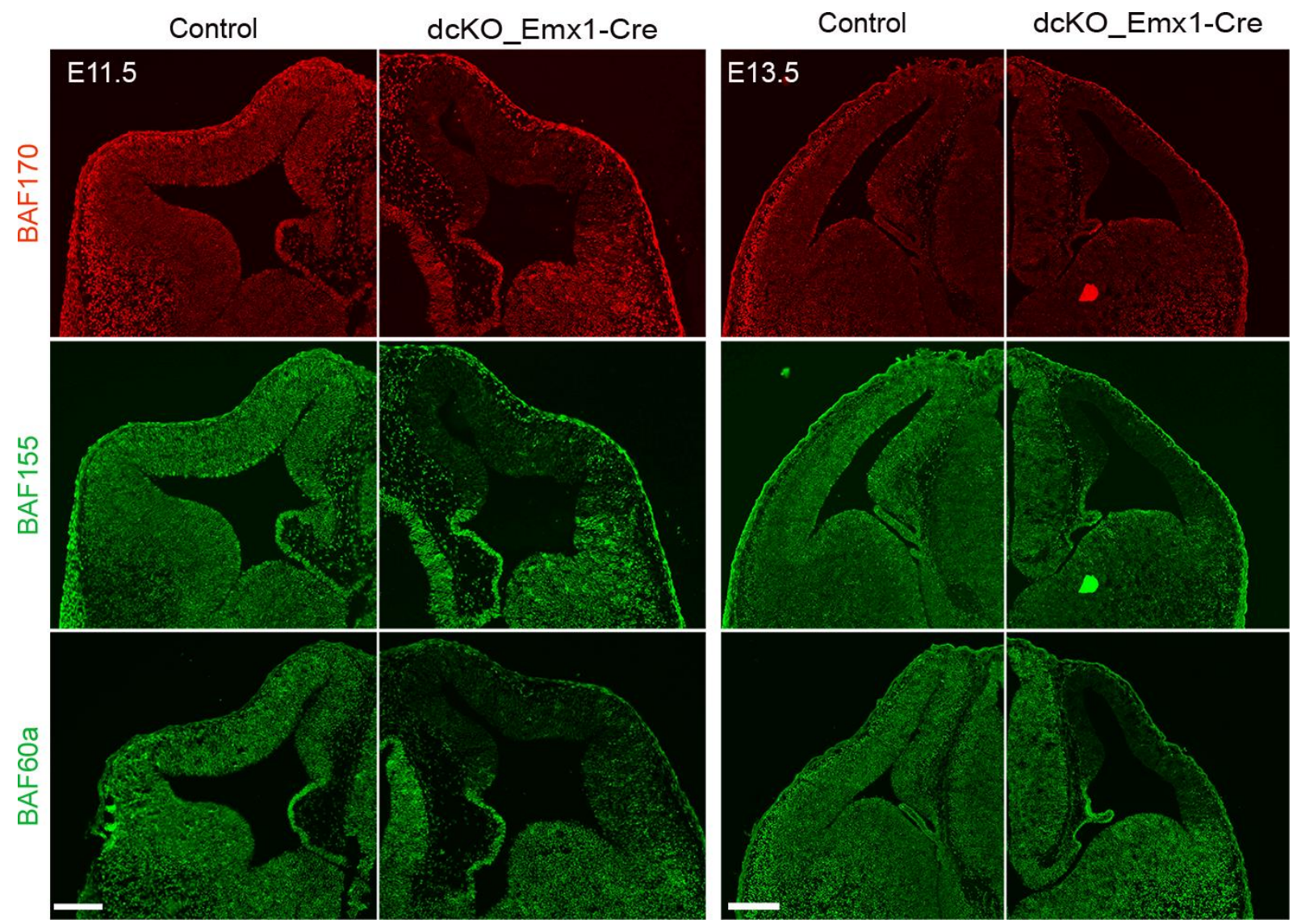

Figure S3.1 Emx1-Cre-mediated deletion of BAF155 and BAF170 causes early loss of BAF complex in the dTel: (A, B) Images of the E11.5 and E13.5 mouse telencephalon immunostained for the BAF complex. Loss of fluorescence signals of the BAF complex subunits BAF155, BAF170, and BAF60a in the dTel of dcKO_Emx1-Cre compared with control indicates of the efficiency of the genetic ablation of the BAF complex via Emx1-Cre-mediated double condition knockout of BAF155 and BAF170. Scale bar: $=100 \mu \mathrm{m}$. 

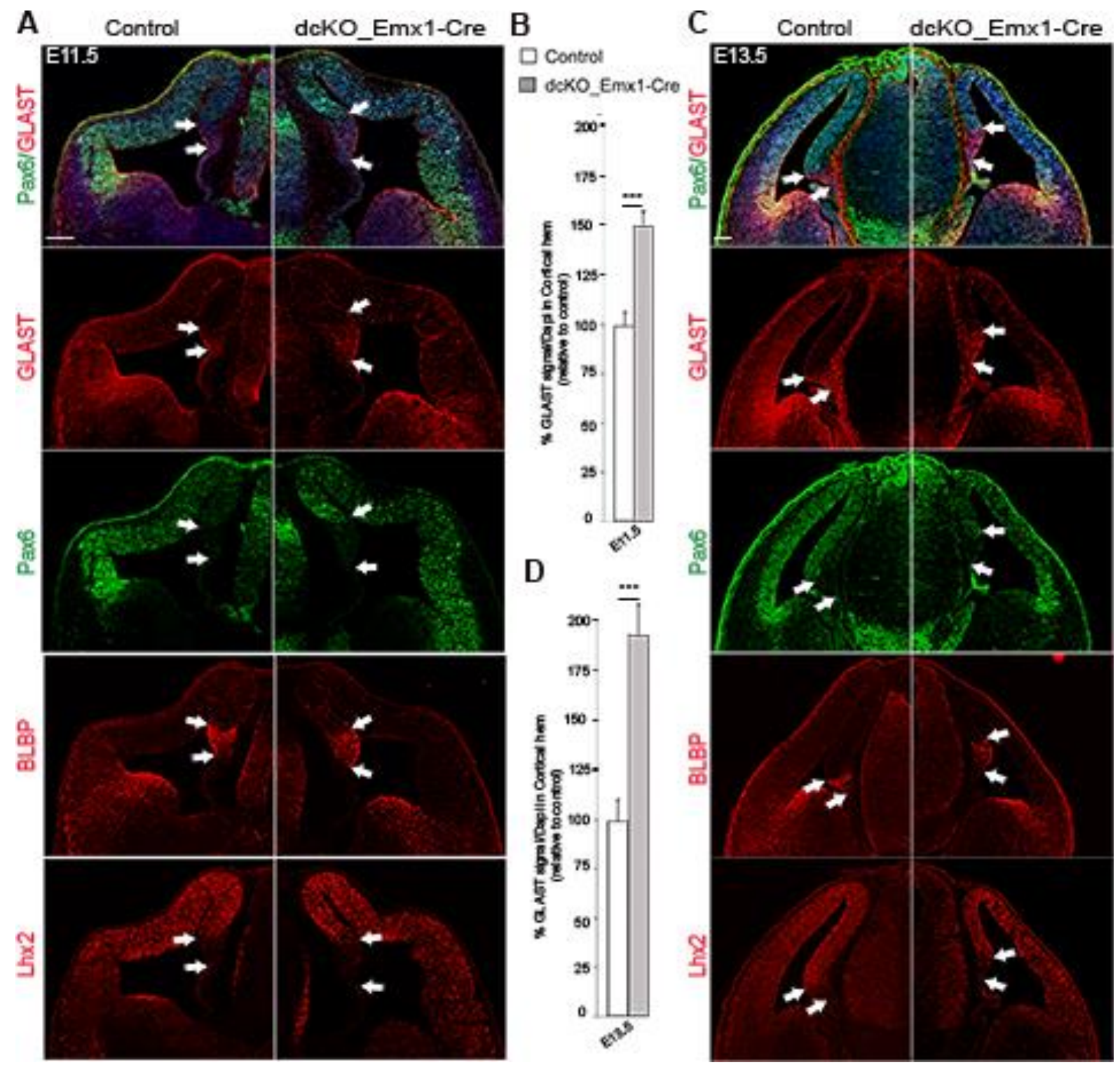

Figure S3.2 Expansion of the cortical hem occurs early in corticogenesis following ablation of BAF complex: (A, C) Images showing the E11.5 (A) and E13.5 (C) mouse telencephalon immunostained for Glast, Blbp, Pax6 and LHX2 to reveal the normal (control) and expanded dcKO_Emx1-Cre cortical hem. Immunostaining with Pax6 and LHX2 antibodies additionally show their gradient expression in the dTel, where Pax6 gradient is lateral ${ }^{\text {high-mediallow }}$ and that of LHX2 is medial ${ }^{\text {high-lateral }}{ }^{\text {low. }}$. The white arrows indicate the full extent of the cortical hem. (B, D) Bar charts indicating the statistical difference between the control and dcKO_Emx1-Cre hem size at E11.5 and E13.5. Sections are counterstained with DAPI (blue), where shown. Unpaired Student's $t$-test: ${ }^{* * *} \mathrm{P}<$ $0.0005 ; n=6$; Scale bar: $=100 \mu \mathrm{m}$. Result are presented as mean \pm SD. 

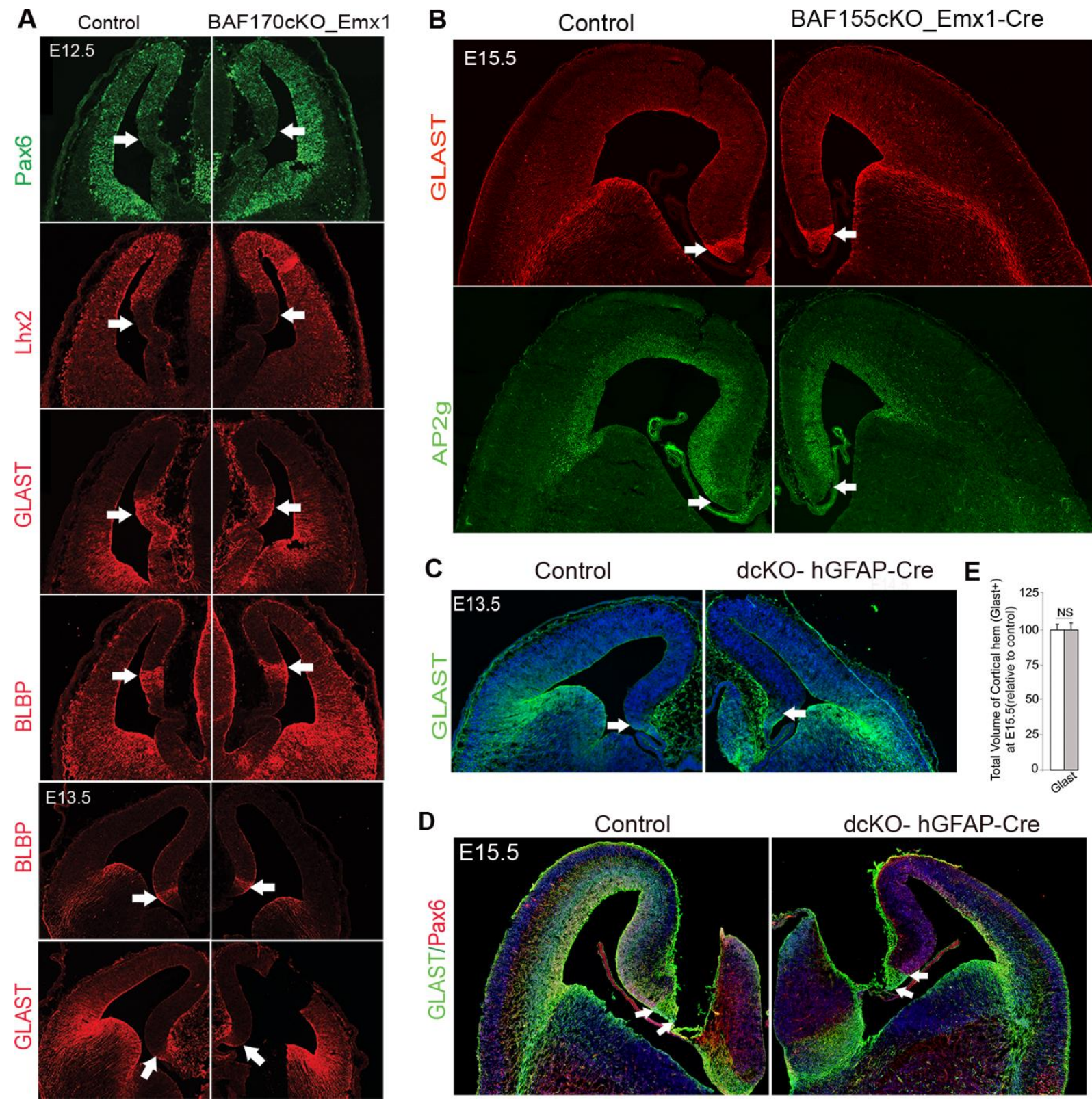

Figure S3.3 Single or late double knockout of BAF155/BAF170 does not cause expansion of the cortical hem: (A, B) Immunomicrographs showing unaltered cortical hem size revealed by hem markers following Emx1-Cre-mediated single conditional knockout of BAF170 (A) or BAF155 (B) in the mouse dTel at the various specified developmental stages. (C, D) Images showing the normal cortical hem size in both control and the dcKO_hGFAP-Cre dTel at E13.5 (C) and (D). (E) Bar charts showing no statistical difference in hem size following late loss of BAF complex. White arrows point to the cortical hem. Sections are counterstained with DAPI (blue). 


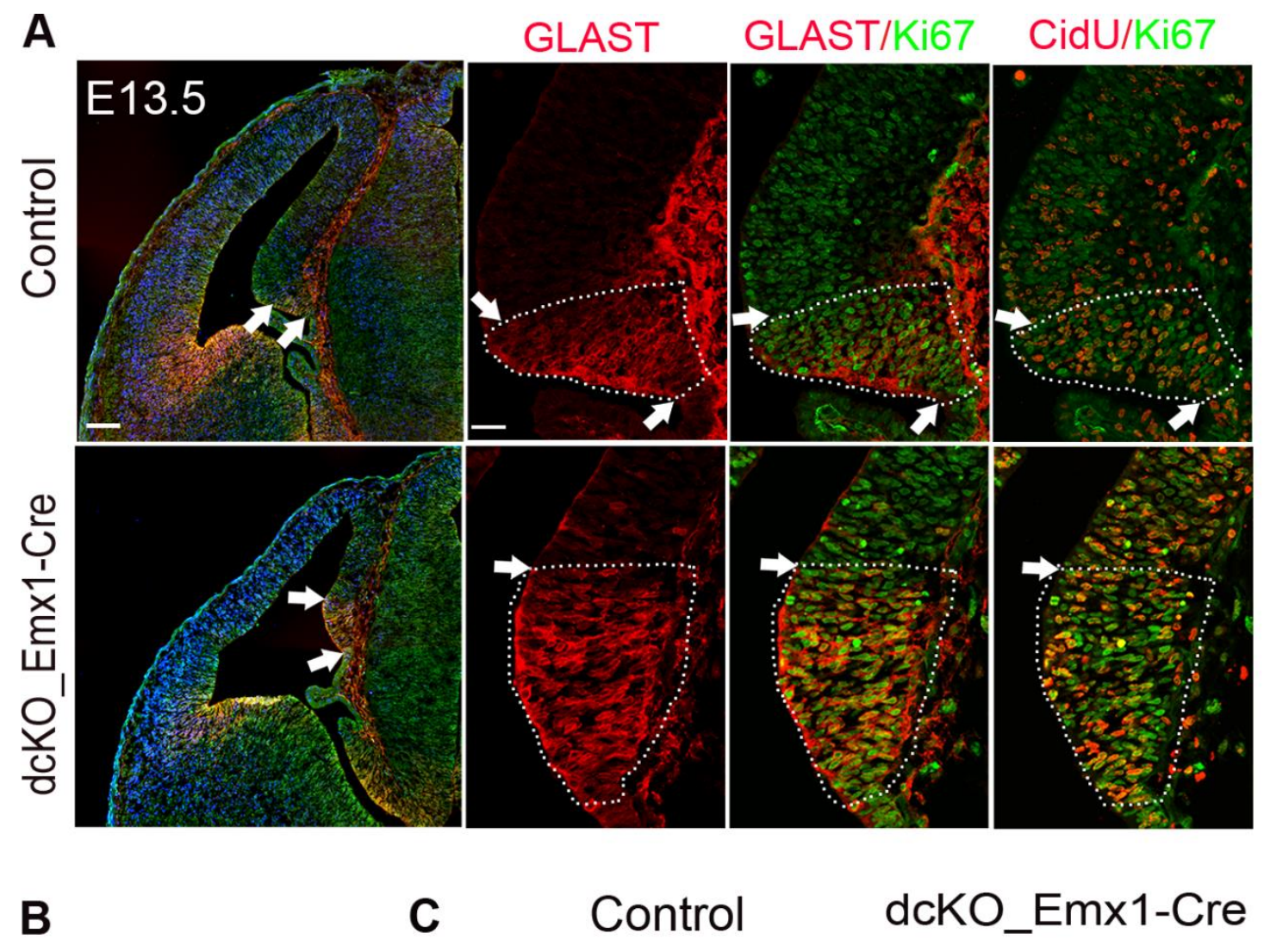

$\square$ Control

$\square$ dcKO_Emx1-Cre
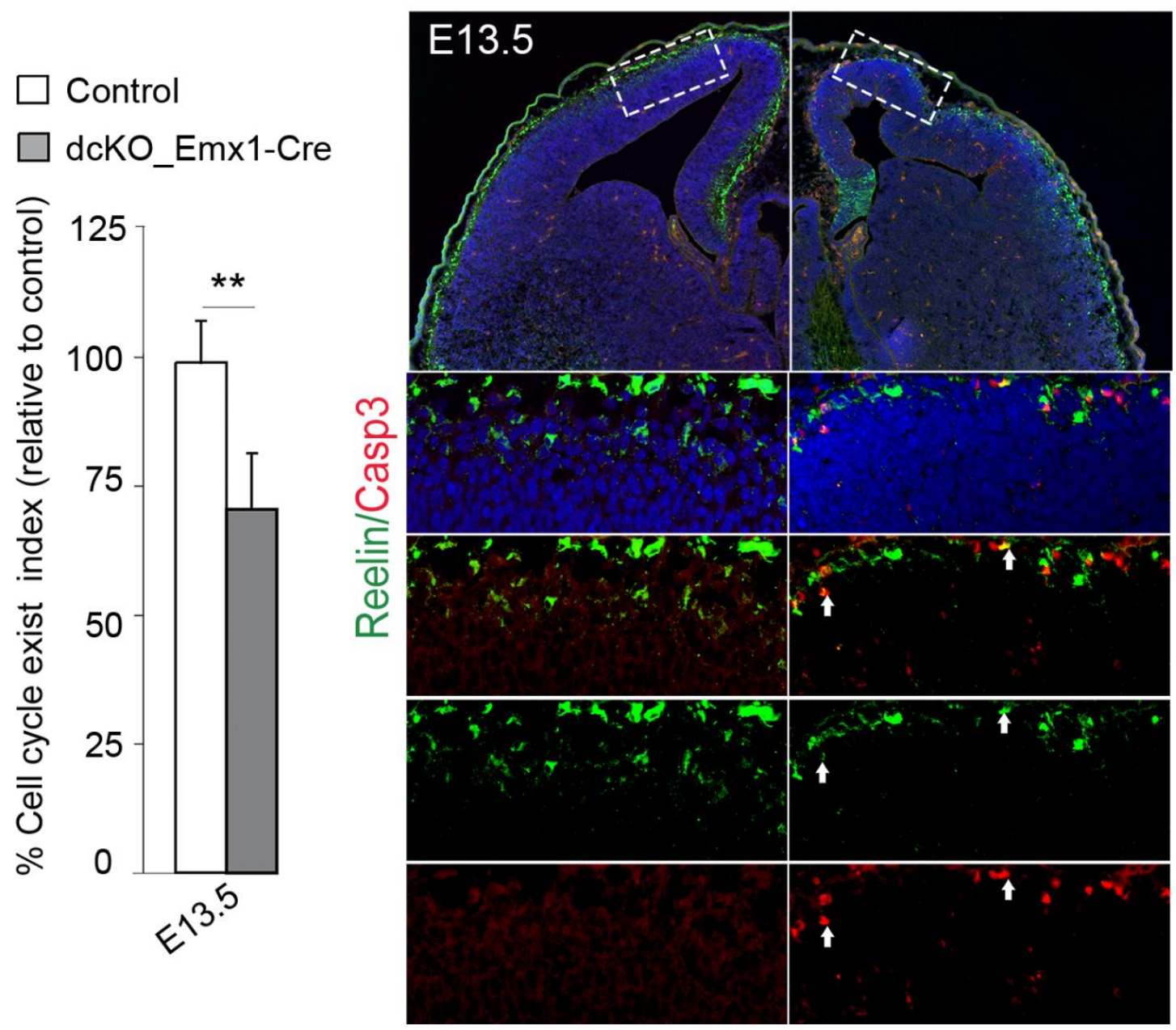
Figure S3.4 Reduced mitotic exit of hem progenitors and increased apoptosis of Reelin+ hem progenies due to loss of BAF complex in dTel: (A) Images showing the cell cycle dynamics of neural progenitors in the E13.5 normal (control) and expanded (BAF complex mutant) cortical hem. A 24-hour single pulse-labeling treatment with $\mathrm{CldU}$ was given before harvesting brains for analysis of progenitor cell cycle kinetics. Immunostaining of CldU marked recently exited or postmitotic cells (in red) and Ki67 antibody was used to stain cycling or proliferating hem progenitor cells (in green). The cortical hem is outlined with white broken lines around the perimeter of the bundles of Glast+ fibers. Upper and lower boundaries of the hem are indicated with white arrows. (B) Bar graph showing statistical difference in the cell cycle exit index between the E13.5 control and dcKO_Emx1-Cre hem neural progenitors. (C) Images of the E13.5 telencephalon immunostained for Reelin to label hem-derived Cajal-Retzius cells and Casp3 to mark apoptotic cells. The frequent colocalization (yellow signal) of Reelin and Casp3 staining (white arrows) is indicative of increased apoptosis of the Cajal-Retzius cells produced by the dcKO_Emx1-Cre cortical hem. Where shown, sections are counterstained with DAPI (blue). Unpaired Student's $t$-test: ${ }^{* *} P<0.005 ; n=4$; Scale bar $=100 \mu \mathrm{m}$. Result are presented as mean \pm SD.

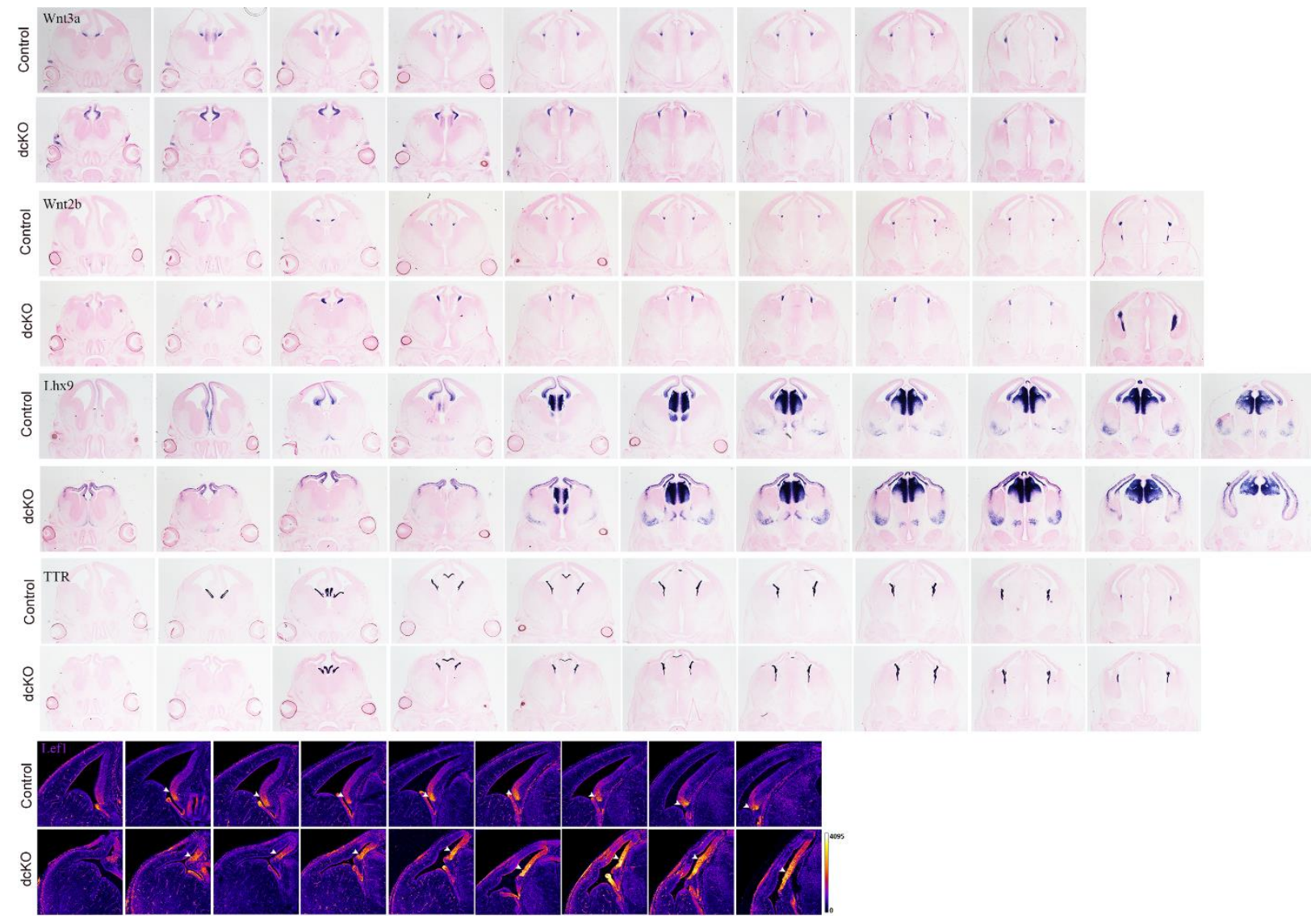

Figure S3.5 The dorsal telencephalon is not properly patterned when BAF complex is deleted in developing mouse cortex: Rostrocaudal serial micrographs showing the E13.5 control and dcKO_Emx1-Cre telencephalon RNA-probed for Wn3a/Wnt2b, TTR, and Lhx9/Lef1 to label the cortical hem, choroid plexus, and parts of the medial cortex, respectively. 


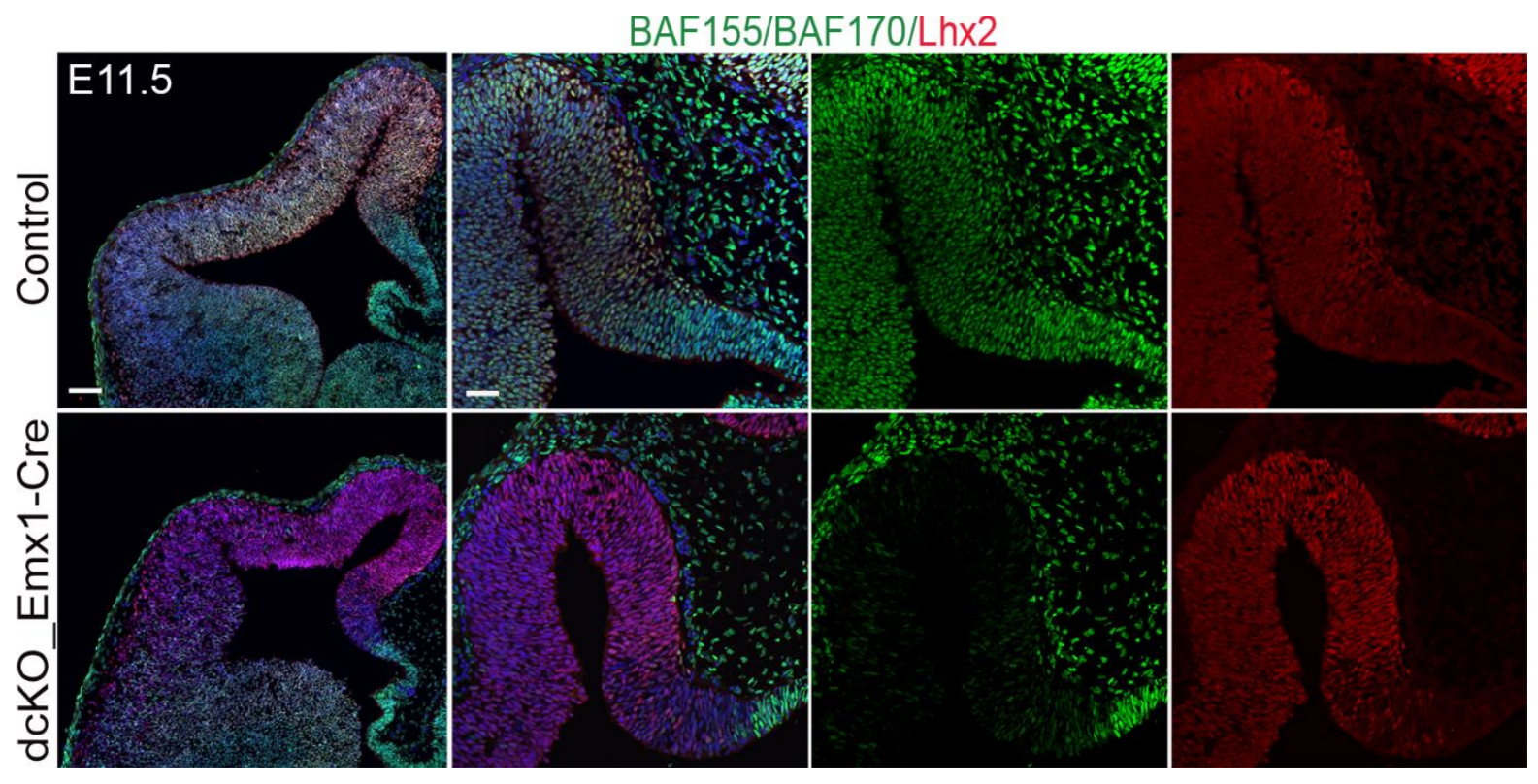

Figure S3.6 Co-expression and/or interaction of BAF complex and LHX2 in the developing cortex: Micrographs showings immunostaining with BAF155, BAF170 and LHX2 antibodies in the E11.5 control and dcKO_Emx1-Cre telencephalon. Interaction of BAF155 and BAF170 (BAF complex) with LHX2 in vivo in the dTel is indicated by the colocalization of their respective protein immunostaining (white signal in control). Close up images of the presumptive cortex and the cortical hem are shown, from left to right, in panels 2, 3 and 4. Where indicated, sections are counterstained with DAPI (blue). Scale bars: $=100$ $\mu \mathrm{m}$ (in overview) and $50 \mu \mathrm{m}$ in close up view.

\subsection{Supplementary experimental procedures}

\subsubsection{Plasmids}

Plasmids used in this study: pCIG2-ires-eGFP, pCIG2-Cre-ires-eGFP (gift from $\mathrm{Dr}$

Francois Guillemot, NIMR London); pGL3-5xLhx2BS-luciferase, pGL3-luciferase, CMV-Lhx2 (Folgueras et al., 2013) (gift from Dr. Elaine Fuchs, the Rockefeller University, New York), Pax6-Luc (Kammandel et al., 1999).

\subsubsection{Antibodies}

The following polyclonal $(\mathrm{pAb})$ and monoclonal $(\mathrm{mAb})$ primary antibodies used in this study were obtained from the indicated commercial sources: AP2 $\gamma$ mouse mAb (1:100; Abcam), BAF170 rabbit pAb (Bethyl), BAF170 rabbit pAb (Sigma), BAF155 rabbit pAb 
(1:20; Santa Cruz), BAF155 mouse mAb (1:100; Santa Cruz), BLBP rabbit pAb (1:200; Chemicon), Casp3 rabbit pAb (1:100; Cell Signaling), GLAST pig pAb (1:500; Frontier), CidU rat pAb (1:100; Accurate), Ki67 rabbit pAb (1:50; Vector), Sox2 mouse mAb (1:100; R\&D Systems), Prox1 rabbit pAb (1:1000; Covance), Pax6 mouse mAb (1:100; Developmental Studies Hybridoma Bank), Pax6 rabbit pAb (1:200; Covance), Tbr2 rabbit pAb (1:200; Abcam), Zbtb20 rabbit pAb (1:50; Sigma), and RFP rabbit pAb (1:10000; Biomol/Rockland), Otx2, BAF60a, Reelin, LHX2 (Folgueras et al., 2013) (gift from Dr. Elaine Fuchs, the Rockefeller University, New York).

Secondary antibodies used were horseradish peroxidase (HRP)-conjugated goat antirabbit IgG (1:10000; Covance), HRP-conjugated goat anti-mouse IgG (1:5000; Covance), HRP-conjugated goat anti-rat IgG (1:10000; Covance), and Alexa 488-, Alexa 568-, Alexa 594- and Alexa 647-conjugated IgG (various species, 1:400; Molecular Probes).

\subsubsection{Generation of dcKO mutants}

To conditionally eliminate BAF155 and BAF170 in the entire telencephalon, early cortical progenitors, or projection neurons, we used the telencephalon-specific FoxG1Cre (Hebert \& McConnell, 2000), early progenitor-active Emx1-Cre (Gorski et al., 2002), or late progenitor-active hGFAP-Cre (Zhuo et al., 2001) mouse lines, respectively. Heterozygous animals (i.e. $B A F 155^{f / /+}, B A F 170^{i /+}, C r e$ ) were used as controls. 


\subsubsection{Marmoset Embryos}

Caesarean Section

For embryos at E74, pregnant mothers were immobilized by intramuscular injection of $25 \mu \mathrm{g} / \mathrm{head}$ of atropine sulfate $(0.5 \mathrm{mg} / \mathrm{mL})$ and $70 \mathrm{mg} / \mathrm{kg}$ of ketamine hydrochloride. Thereafter, animals were anesthetized by inhalation of $1-3 \%$ of isoflurane via a ventilation mask. Anesthetization management was performed by spontaneous respiration during the operation, monitoring the heart rate and the arterial oxygen saturation. The uterus was exteriorized following midline laparotomy, and the proximal end of the uterus was incised for the Caesarean section. After the Caesarean section, the uterus, abdominal muscles, and skin were sutured. Embryonic brains were dissected in ice-cold phosphate-buffered saline (PBS), and transferred to fixative after removal of the meninges.

\subsubsection{Tissue processing}

After dissection, the marmoset E74 brains were immersed in 4\% paraformaldehyde (PFA) (w/v) in PBS and left in fixative for $24 \mathrm{~h}$ at $4^{\circ} \mathrm{C}$ with mild agitation. After fixation, the brains were kept at $4^{\circ} \mathrm{C}$ in a mixture of 3 parts PBS containing $0.01 \%$ NaN3 and 1 part 4\% PFA in $120 \mathrm{mM}$ sodium phosphate buffer with $\mathrm{pH} 7.4$ until processed.

For cryosectioning, fixed brains (marmoset, mouse) were cryoprotected, first in 15\% sucrose in PBS and then in $30 \%$ sucrose in PBS at $4^{\circ} \mathrm{C}$. Brains were embedded in Tissue-Tek (Sakura Finetek) and stored at $-20^{\circ} \mathrm{C}$. Sections were cut at $16 \mu \mathrm{m}$ and stored at $-20^{\circ} \mathrm{C}$. 


\subsubsection{Western blot analyses}

Western blot analyses were performed as described previously (Tuoc \& Stoykova, 2008).

\subsubsection{Co-immunoprecipitation and mass spectrometry (CoIP/MS)}

BAF155 and BAF170 interaction analyses were performed using the neural stem cell line, NS5 (Conti et al., 2005), and embryonic telencephalic tissue at E13.5 and E17.5. Tissues were dissected and minced in cold PBS and then washed twice with PBS. Equivalent amounts of cells from one embryo were lysed for $30 \mathrm{~min}$ in $1 \mathrm{ml}$ RIPA buffer containing a proteinase inhibitor cocktail (Roche) and DNase. All steps were performed at $4^{\circ} \mathrm{C}$. Lysates were centrifuged for $10 \mathrm{~min}$ at $13,000 \mathrm{rpm}$ to sediment out non-lysed tissues. The supernatant was pre-cleared by incubating with normal mouse IgG together with protein A/G-agarose beads, as described by the manufacturer (sc-2003; Santa Cruz). Interacting proteins were immunoprecipitated by incubating pre-cleared supernatant with rabbit anti-BAF155 and anti-BAF170 antibodies and A/G-agarose beads. The beads were then washed first with $500 \mu$ cold RIPA buffer (three times for 5 min each) and then with $40 \mu \mathrm{l}$ of elution buffer $\left(2.5 \mu \mathrm{l} 20 \% \mathrm{SDS}, 5 \mu \mathrm{l} 1 \mathrm{M} \mathrm{NaHCO}_{3}\right.$, $42.5 \mu$ double-distilled $\mathrm{H}_{2} \mathrm{O}$ ) for 15 min at room temperature.

For MS analyses (performed in the department of Prof. Dr. Henning Urlaub), samples were suspended in NuPage loading buffer and resolved on commercial SDS polyacrylamide gels (Novex NuPage Bis-Tris gel, 4-12\% gradient; Invitrogen). Individual lanes were then cut into six squares for MS analysis. The parameters for the identification of proteins were set to the following values: limit, 95\% probability of detection; limit of unique peptides detected, 1; and threshold detection probability of peptides, $80 \%$. 
The list of BAF155- and BAF170-interacting proteins revealed by MS analysis was obtained by subtracting nonspecific interactions with IgG in IPs and in telencephalic tissues from BAF155-null (BAF155cKO_FoxG1-Cre) and BAF170-null (BAF170cKO_FoxG1-Cre) mutants. The first set of controls excludes nonspecific binding to the antibody, and the second excludes nonspecific interactions that could possibly be precipitated by either the anti-BAF155 or anti-BAF170 antibody. 


\section{CHAPTER 4: Loss of BAF complex in developing cortex perturbs neuronal migration and process outgrowth in a WNT signaling- dependent manner}

Godwin Sokpor, Cemil Kerimoglu, Linh Pham, Huong Nguyen, Joachim Rosenbusch, Robin Wagener, Anastassia Stoykova, Jochen F. Staiger, Tran Tuoc

This chapter is a prepared manuscript. Its submission for publication is forthcoming.

Personal contributions: I performed most of the phenotype characterization, and all the statistical analyses. RNA sequencing figures were generated by me. I drafted and further modified the manuscript together with the project supervisor.

\subsection{Abstract}

Radial neuronal migration is an essential neurodevelopmental event central to cortical laminar organization. Cortical neurons mainly use glial fiber guides, cell adhesion dynamics, and cytoskeletal remodeling, among other discrete processes, to radially trek from their birthplace to final layer positions. Dysregulated radial migration can engender cortical mis-lamination, leading to neurodevelopmental disorders. Epigenetic factors, including chromatin remodelers are emerging as formidable regulators of corticogenesis. Notably, the chromatin remodeler BAF complex has been shown to regulate several aspects of cortical histogenesis. Nonetheless, our understanding of how BAF complex regulates neuronal migration is limited. Here, we report that BAF complex is required for neuron migration and process elaboration during cortical 
development. Ablation of BAF complex disturbed glial fiber formation and cell adhesion critical for radial migration; culminating in cortical laminar malformation. Of note, BAF complex inactivation caused defective neuronal polarization resulting in diminished multipolar-to-bipolar transition and eventual loss of callosal neuron axonal projection, attributable to WNT hyperactivity. Mechanistically, the BAF complex may be a modulator of WNT signaling activity to drive glial fiber-dependent neuronal migration, neurite formation, and cortical lamination. Overall, BAF complex has been identified to be crucial for cortical morphogenesis through instructing multiple aspects of radial migration in a WNT signaling-dependent manner.

\subsection{Introduction}

Early and late patterning events, including specification and diversification of neural progenitors, and neuronal migration, afford gross and subtle anatomical and functional cortical areas establishment during forebrain development (Silva et al., 2019). For the most part, neuronal migration is the critical process that ensures proper placement of isogenic groups of neurons into their predetermined cortical laminae during morphogenesis of the cortex. Hence, in the event of neuronal misplacement due to abnormal migration, the cortex is mis-laminated and the ectopic neurons become susceptible to developmental anomalies, including abnormal differentiation, incorrect neurite formation and synaptogenesis, and dysregulated cell death which can culminate in many neurodevelopmental disorders (Valiente \& Marin, 2010; Evsyukova et al., 2013).

The bona fide excitatory neurons generated by radial glia $(R G)$ cells in the ventricular zone (VZ) or by intermediate progenitors in the subventricular zone (SVZ) of the developing cortex make incredibly long and challenging navigations from their place of 
birth to specified layers in the cortical plate $(\mathrm{CP})$ to constituent what is referred to as radial migration (Noctor et al., 2001; Noctor et al., 2002; Pontious et al., 2008). Radial migration can occur in the form of somal translocation or locomotion (Nadarajah et al., 2001). During somal translocation, mainly used by early-born neurons, the nascent neuron elaborates a long leading process anchored at the pial basement membrane. By means of progressive traction force generated by shortening of the long leading process, the soma of the neuron is continually translocated to be placed in its designated cortical lamina (Miyata et al., 2001; Nadarajah et al., 2001).

Locomotion on the other hand is a more complex and multiphasic process. Unlike somal translocation, it depends on the molecular and structural guidance of RG fibers needed for the neuronal movement profiles that largely contribute to the formation of superficial neocortical layers (Rakic, 1972). The locomoting neuron, mainly late-born, displays morphological fluidity in the course of trekking. Notably, during locomotion, the newborn neuron briefly attaches to its mother glial fiber or the adjoining fiber and actively moves with a bipolar morphology into the lower intermediate zone (IZ). In the IZ, the bipolar neuron disengages from the glial fiber to momentarily pause radial migration. It then transitions to or adopts a multipolar morphology with which it makes undefined micro-movements in effort to collect directional cues for subsequent radial (oriented) migration. Upon adequate molecular conditioning and transient NMDA receptor-mediated glutamatergic synaptic stimulation, the multipolar neuron then switches back to bipolar morphology in the vicinity of the upper IZ and re-attaches to the glial fiber to resume locomotion to its final destination in the CP (Kriegstein \& Noctor, 2004; Noctor et al., 2004; Inoue et al., 2014; Mizutani, 2018; Ohtaka-Maruyama et al., 2018). The bipolar neuron characteristically extends a pia-directed leading process, the dendrite-to-be, and a trailing process towards the VZ which becomes the 
future axon (Rakic et al., 1996). Adopting the appropriate neuronal morphology or polarity is a key determinate of successful radial migration and cortical morphogenesis, which can lead to neurodevelopmental anomalies when perturbed (Hakanen et al., 2019). Locomotion ends in the CP by detachment of the migrating neuron from the glial fiber to be properly positioned in its home layer via somal translocation (Nadarajah et al., 2001).

Indeed, neuronal migration (locomotion) is a complex cell biological process which must be under tight molecular regulation. As such, myriad of factors, including transcriptional and signaling factors have been identified to spatiotemporally regulate various aspects of cortical neuron migration (reviewed in (Heng et al., 2007; Marin et al., 2010; Evsyukova et al., 2013)). Notably, it has been shown in seminal studies and reviews that the formation and maintenance of $R G$ fibers, and related neuronal cell adhesion dynamics are tightly regulated during radial migration (Anton et al., 1997; Anton et al., 1999; Elias et al., 2007; Kawauchi et al., 2010; Shikanai et al., 2011; Sild \& Ruthazer, 2011; Valiente et al., 2011; Solecki, 2012; Desai et al., 2013; Evsyukova et al., 2013; Schmid et al., 2014; Tonosaki et al., 2014; Jinnou et al., 2018; Louhivuori et al., 2018; Schaffer et al., 2018; Zhang et al., 2019).

Epigenetic factors have lately been at the center stage of neurodevelopmental regulation after previous underestimation of their phenomenal role in orchestrating neural development. Emerging among these epigenetic regulators are the chromatin remodelers which can redesign the epigenetic landscape to influence gene expression and related cell biological events through direct alteration of chromatin structure and/or the recruitment of other epigenetic or transcriptional cofactors during cortical development (Sokpor et al., 2018). 
The Brg1/Brm-associated factor (BAF) complex, a mammalian version of the yeast SWI/SNF complex, is a multi-subunit protein complex which primarily functions as a chromatin remodeler (Clapier et al., 2017) and has been shown in recent years to be indispensable for neural development (Son \& Crabtree, 2014; Sokpor et al., 2017; Sokpor et al., 2018). During cortical development, the BAF complex regulates key processes such as specification, proliferation, differentiation and functional maturation of cortical progenitors or postmitotic neurons (Son \& Crabtree, 2014; Sokpor et al., 2017; Sokpor et al., 2018). Notably, the BAF complex subunits can be reconstituted to form cell type specific variants that have unique functional effects. For example, the BAF complex in neural progenitors (npBAF) is compositionally and functionally distinct from that in postmitotic neurons (nBAF) (Lessard et al., 2007; Wu et al., 2007; Kadoch et al., 2013; Tuoc et al., 2013; Bachmann et al., 2016).

Although chromatin remodelers, including some BAF subunits, have been reported to regulate neuronal migration in the developing mammalian cortex (Nott et al., 2013; Wiegreffe et al., 2015; Nitarska et al., 2016; Xu et al., 2018) and in worm neural tissue (Weinberg et al., 2013), how they mechanistically control neuronal migration during brain development is far from clear. In this current study, we aimed at clearly defining the molecular and cellular mechanisms through which the BAF complex orchestrates migration of excitatory/projection neurons in the developing cortex.

To that end, we abolished the BAF complex in early and late cortical progenitors and specifically in postmitotic neurons to investigation how BAF complexes influence neuronal migration during corticogenesis. From our molecular and cellular analyses of the BAF complex mutant (knockout or knockdown) cortex, it was evident that neurons fail to migrate properly in the absence of BAF complex functionality. As a result, cortical 
neurons are misplaced in the mutant cortex leading to abnormal cortical cytoarchitectonic and concomitant laminar malformation. The BAF complex-ablated cortical neuron is incapable of proper radial migration because of loss of glial fiber guides and adhesion molecules, defective formation of processes required for locomotion, and abnormal WNT signaling activity. The said altered factors and process are however critical for correct radial migration, and are tightly regulated by many other factors during cortical morphogenesis (Reviewed in (Evsyukova et al., 2013)).

Our findings that BAF complex ablation in the developing mouse forebrain leads to cortical mis-lamination, agenesis of corpus callosum, and gross reduction in cortical size being rescuable by manipulation of WNT signaling, may have therapeutic applicability for some reported clinical neurodevelopmental anomalies liked to BAF complex subunit mutations (Sokpor et al., 2017).

\subsection{Results}

\subsubsection{BAF complex-deficient early developing cortex lacks critical neuronal migration factors and appears severely malformed}

In order to study the role of BAF complex in RG fiber-dependent neuronal migration during early development of the cortex, we conditionally ablated the BAF complex function in cortical RGs at the onset of neurogenesis onward. This was done by crossing mice double floxed for the BAF complex subunits BAF155 (Choi et al., 2012) and BAF170 (Tuoc et al., 2013) with the early-acting (E10.5) dorsal telencephalonspecific Emx1-Cre (Gorski et al., 2002) to generate the double conditional knockout (dcKO) BAF mutant cortex, herewith referred to as dcKO_Emx1-Cre. (Fig. S4.1). We previously identified that the BAF complex function is adequately abolished by double 
deletion of its scaffolding subunits BAF155 and BAF170. In the absence of BAF155 and BAF170, the entire BAF complex stability is compromised. This leads to disassembly of other components (subunits) of the complex, making them susceptible to proteasomal degradation and attendant functional inactivation of the entire BAF complex (Narayanan et al., 2015; Bachmann et al., 2016; Nguyen et al., 2016; Nguyen et al., 2018).

Examination of the E13.5 early developing dcKO_Emx1-Cre cortex, immunohistochemically stained for HuCD (a pan-neuronal marker), revealed reduction in cortical size due to decreased differentiation of RGs and increased apoptosis as compared with the control (wild-type) cortex (Fig. 4.1A, left vertical panel; (Narayanan et al., 2015)). At E15.5, in comparison to the control cortex, the dcKO_Emx1-Cre cortex appeared dramatically malformed with striking perturbations in progenitor pool and neuronal generation as indicated by Pax6 and Tbr1 immunohistochemistry, respectively (Fig. 4.1A, middle vertical panel). Normally, by E15.5, cortical neurogenesis is at its peak and the first wave of early-born/layer 6 (L6) Tbr1+ neurons have already successfully migrated into the $\mathrm{CP}$ to make the first clearly demarcated cortical lamina (Fig. 4.1A; (Molyneaux et al., 2007; Gaspard et al., 2008)). At higher magnification, the dcKO_Emx1-Cre cortex showed a cocktail of Pax6+RGs and Tbr1+ L6 neurons which are rather discernably separated by the intervening IZ in the corresponding control cortex (Fig. 4.1A, right vertical panel). This observation convey an impression of neuronal migration disturbance amid other cortical developmental anomalies imputable to loss of BAF complex in the early stages of corticogenesis.

When we re-examined our previously generated RNA sequencing data in which 1723 genes were downregulated and 102 genes upregulated in the E12.5 dcKO_Emx1-Cre 
cortex (Fig. 4.1B; (Narayanan et al., 2015), we found in our gene ontology analysis that key pathways involved in cell polarity via cytoskeletal remodeling, cell adhesion, neuronal cell migration and neurite formation were significantly downregulated compared with control cortex (Fig. 4.1C). Given that these categories of factors play crucial roles in oriented-neuronal migration (locomotion) to afford proper cortical lamination, we were interested in determining whether truly the dcKO_Emx1-Cre cortex exhibits deficits in such factors necessary for radial neuronal migration. In that direction, we immunostained Nestin in the E13.5 dcKO_Emx1-Cre cortex. Classically, Nestin staining reveals RG cells and particularly their fiber elaboration that traverses the developing cortex from the VZ to the pial surface, where they are anchored to the basement membrane by branched end feet (Control in Fig. 4.1D). The Nestin+ RG fiber acts as scaffold to which nascent postmitotic neurons attach in order to radially migrate from their place of birth to their final destination (cortical layer) in the CP. Strikingly, we found that such Nestin+ fibers are massively lost in the E13.5 dcKO_Emx1-Cre cortex (Fig. 4.1D/E).

To substantiate the observed loss of Nestin+ fibers and/or cells in the dcKO_Emx1Cre cortex, we also checked for the integrity of cell adhesion at the cortical apical surface that anchors the Nestin expression RG cells in the VZ of the developing cortex. For this, we examined $a$-Catenin expression, a typical adhesion protein intensely expressed at the apical surface, and noticed significant depletion of this apical adherens junctional protein at the E13.5 dcKO_Emx1-Cre cortical ventricular surface. The intra-cortical expression of $\alpha$-Catenin was also reduced in the E13.5 dcKO_Emx1Cre cortex as compared with control (Fig. 4.1D/F). This result was also in consonance with the observed downregulation of cell-cell interaction/adhesion category of gene expression captured in our gene ontology analysis (Fig. 4.1C). Notably, apart from 
being a component of the adherens junction belt at the apical surface of the VZ, $\alpha$ Catenin is also crucial for adhesion of migrating neurons to glial fibers for locomotion by forming indirect links with the internal cytoskeleton (Drees et al., 2005; Elias et al., 2007). Therefore, loss of cortical $\alpha$-Catenin can on its own disturb neuronal migration and cortical morphogenesis during forebrain development (Fig. 4.1A; (Schmid et al., 2014; Schaffer et al., 2018).

Altogether, we obtained foundational evidence indicating dysregulated migration of cortical projection neurons following inactivation of BAF complex functionality in the cortical neuroepithelium of the early developing mouse forebrain. The apparent perturbation of neuronal migration may partly contribute to the severe cortical dysgenesis of the early developing BAF complex mutant cortex. 


\section{A}

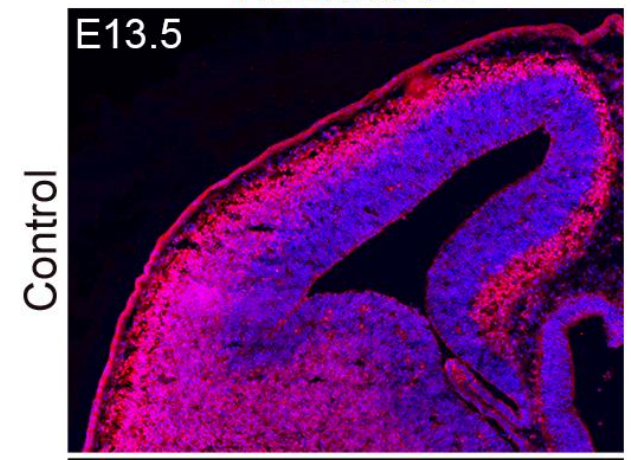

HuCD/DAPI

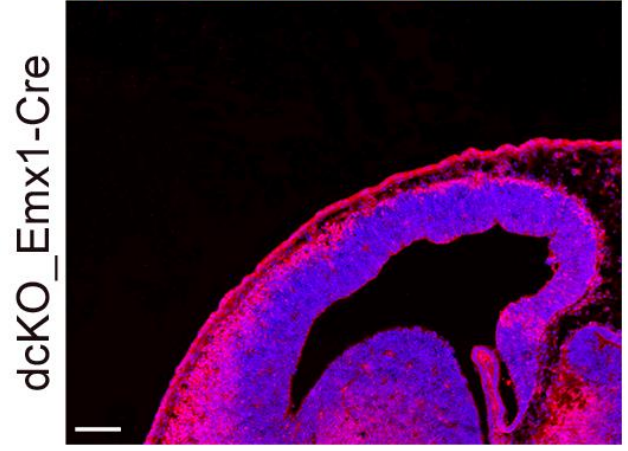

B
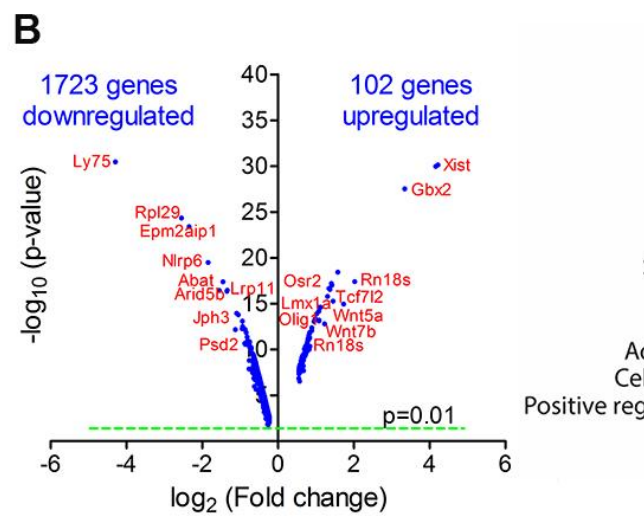

$$
\text { D }
$$
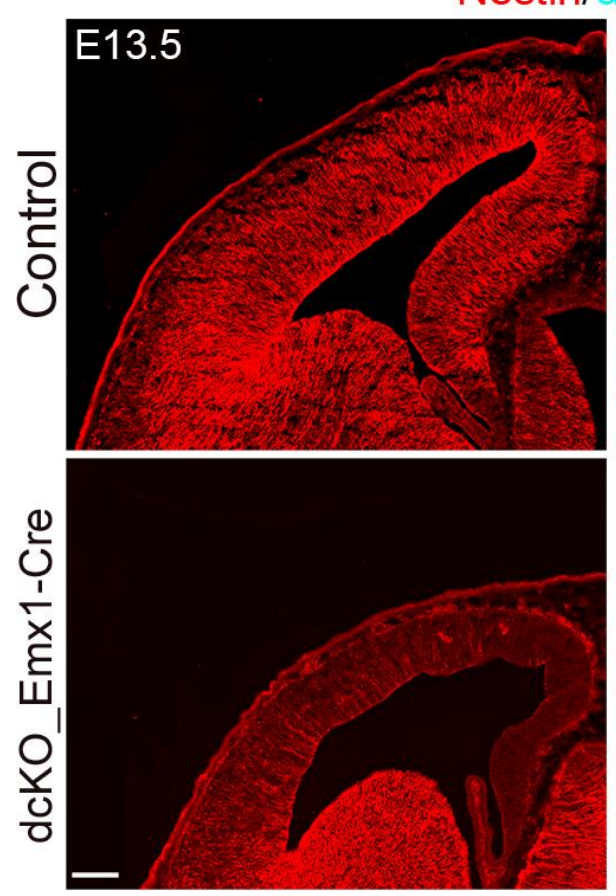
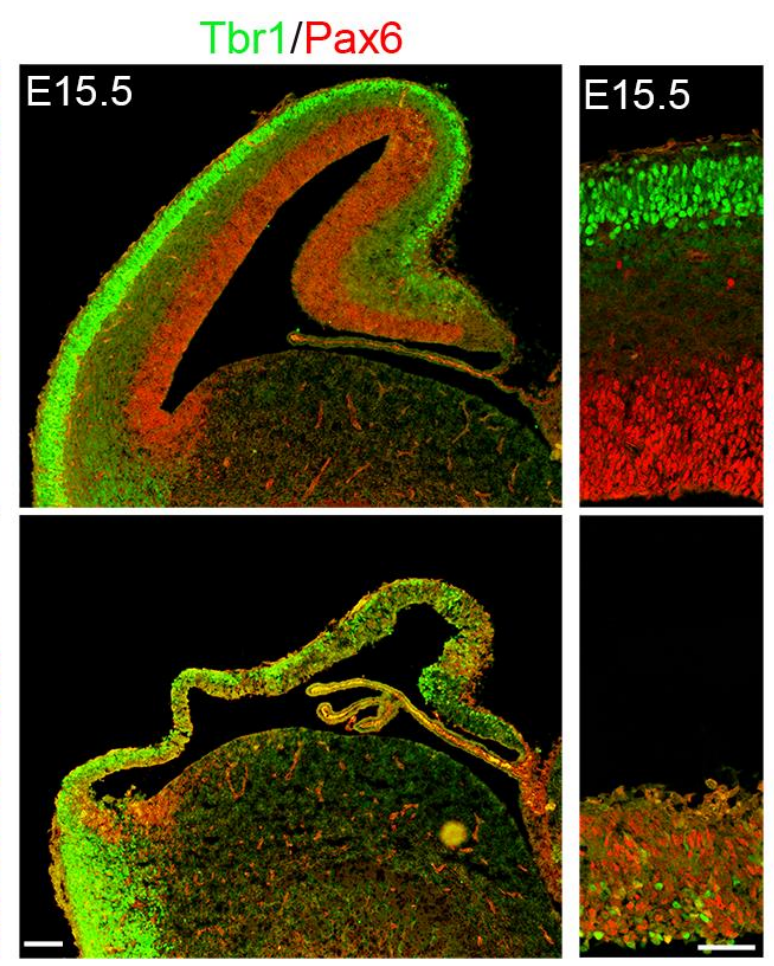

C $\square d c K O \_E m \times 1-C r e ~ v s$ Control

$0 \quad-2 \quad \log (\mathrm{p}$-value)

Locomotion
Cytoskeletal part

Regulation of cell migration

Cell-cell adherens junction

Single organism cell adhesion

Basement membrane
toskeleton organization

Cytoskeleton organization

Adherens junction organization

Cell-substrate junction assembly

Microtubule

Plasma membrane

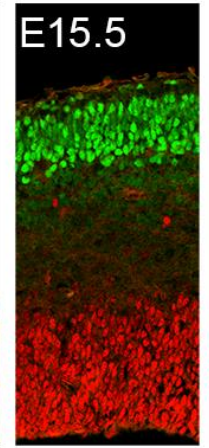

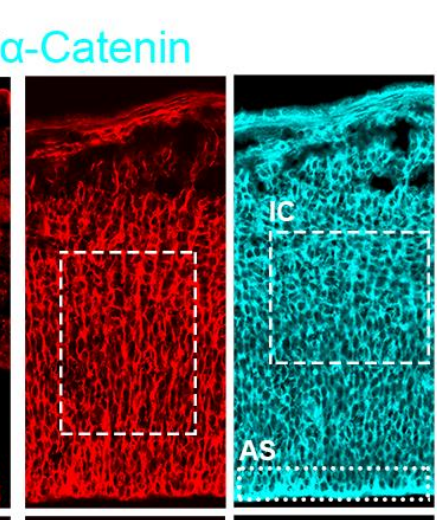

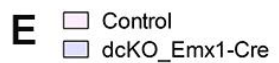
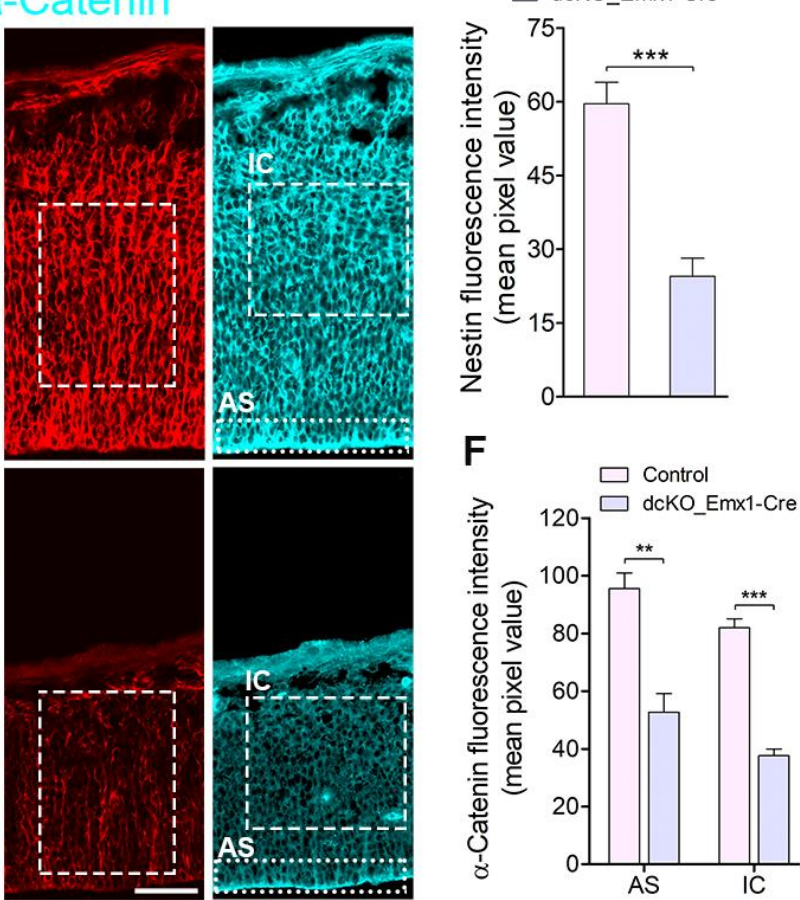
Figure 4.1 BAF complex inactivation causes massive cortical malformation partly due to defective neuronal migration in the early developing cortex: (A) Immunohistochemical staining of coronal sections of E13.5 and E15.5 control (wild type) and dcKO_Emx1-Cre mouse cortex with antibodies against the pan-neuronal marker protein HuCD, early-born layer 6 neuronal marker Tbr1, and the neural stem/progenitor cell marker Pax6. (B) Volcano plot showing genes downregulated and upregulated in the dcKO_Emx1-Cre cortex. Examples of top altered genes are indicated. The total number of genes downregulated and upregulated in the E12.5 dcKO_Emx1-Cre cortex as compared with control are indicated. (C) Graphical representation of gene ontology analysis of RNA-seq results in the E12.5 dcKO_Emx1-Cre cortex versus (vs) control showing downregulation of migration-related gene categories. (D) Sections of E13.5 control and dcKO_Emx1-Cre cortex immunostained for the glial fiber protein Nestin and the cell adhesion protein $\alpha$-Catenin. White rectangular inserts represent respective cortical arears comparatively selected for quantifications. (E, F) Simple (E) and grouped (F) bar charts showing quantification of Nestin and $\alpha$-Catenin expression, respectively, in the E13.5 control and dcKO_Emx1-Cre cortex. Where shown, sections are counterstained with DAPI (blue). Unpaired Student's $t$-test was used to test for statistical significance: ${ }^{* \star} p<0.005,{ }^{* * *} p<0.0005 ; n=4$; Scale bars: $=100 \mu \mathrm{m}$ and $50 \mu \mathrm{m}$ in overview and zoomed images, respectively. Results are presented as mean \pm SEM. Abbreviations: Intracortical (IC), Apical surface (AS).

\subsubsection{Loss of BAF complex later in corticogenesis results in impaired neuronal migration leading to cortical mis-lamination and dysgenesis}

Since the cortical mass is dramatically lost when BAF complex is abolished early in cortical development (Fig. 4.1A; (Narayanan et al., 2015)), we were unable to study neuronal migration further and comprehensively in the dcKO_Emx1-Cre forebrain. This warranted our choice of another BAF complex mutagenic strategy that allowed us to delete the BAF complex at a later stage of corticogenesis. Thus, we generated the dcKO_hGFAP-Cre forebrain model in which BAF155 and BAF170 are deleted predominately in the $\mathrm{VZ}$ progenitors of the developing cortex to achieve ablation of the entire BAF complex (Fig. S1; (Nguyen et al., 2018)). Since the hGFAP-Cre is relatively late-acting, with the earliest activity detected around E13.5 (Zhuo et al., 2001; Nguyen et al., 2018), we were able to lessen the impact of loss of BAF complex on cortical morphogenesis compared with that caused in the dcKO_Emx1-Cre (Fig. 4.2 vs. Fig. 4.1). Therefore, the dcKO_hGFAP-Cre model allowed us to study neuronal migration at late embryonic and early postnatal stages of cortical development with fairly preserved cortical integrity in the absence of BAF complex. 
We characterized the migration phenotype in the E17.5 cortex, by which stage the knockout effect under the hGFAP-Cre activity is fully established in the entire cortex (Nguyen et al., 2018). We started by reanalyzing our previously generated RNA sequencing data from the E17.5 cortex in which 1329 genes and 1195 genes were downregulated and upregulated, respectively (Fig. 4.2A). Again, we screened for gene categories implicated in the regulation of neuron migration in the cortex. Consistent with our observations in the dcKO_Emx1-Cre cortex (Fig. 4.1B/C), we found several gene pathways involved in cell polarity establishment and adhesion downregulated in the dcKO_hGFAP-Cre cortex (Fig. 4.2B). We then immunohistochemically checked for the adhesion protein $\alpha-C a t e n i n$ and the Nestin+ RG scaffolds in the E17.5 control and dcKO_hGFAP-Cre cortex. As expected, we found demonstrable depletion of RG fibers and reduction in the apical and intra-cortical distribution of ( $\alpha$-Catenin-mediated) cell adhesion in the dcKO_hGFAP-Cre cortex as compared with control (Fig. 4.2C-E; (Nguyen et al., 2018)). Interestingly, there was no commensurate reduction in Pax6/Sox2+ progenitors in the dcKO_hGFAP-Cre germinal zones despite the loss of Nestin+ fibers. Rather, the neural stem cell pool was increased, although at the expense of neurogenesis, in the dcKO_hGFAP-Cre developing cortex (Nguyen et al., 2018).

The neuronal migration phenotype in the E17.5 dcKO_hGFAP-Cre and control developing cortex was assessed by applying antibodies against the lower layer (L5) cortical neuron marker protein Ctip2 (Arlotta et al., 2005; Gaspard et al., 2008) and the upper cortical layer neuron marker protein Cux1 (Nieto et al., 2004), which revealed that loss of BAF complex at later-stage of embryonic corticogenesis severely disturbed neuronal migration (Fig. 4.2F). We also checked the cortical neuron migration phenotype just after birth (P0), by which time majority of Tbr1+ and Ctip2+ lower layer 
neurons have largely completed somal translocation or locomotion to properly settle in their respective layers (Nadarajah et al., 2001; Marin \& Rubenstein, 2003; Molyneaux et al., 2007). Unlike Cux1 or Brn2+ neurons, Tbr1+ and Ctip2+ neurons are normally born in the dcKO_hGFAP-Cre cortex (Nguyen et al., 2018). Our immunohistological and quantitative analysis indicated significant disturbance in Ctip2+ L5 neuronal distribution in the P0 dcKO_hGFAP-Cre cortex as compared with control (Fig. 4.2G/H). Although, most of the Tbr1+ L6 neurons migrated out of the germinal zones and IZ, they appear to have over-migrated, making them locate in upper layer domains in the PO dcKO_hGFAP-Cre cortex as compared with control (Fig. 4.2G/I).

Together, these results strongly implicate the role of the BAF complex in orchestrating radial migration of cortical neurons and proper development of the cortex. However, just like in the dcKO_Emx1-Cre model, the migration phenotype in the dcKO_hGFAPCre cortex co-exists with disruption in neural progenitor cell proliferation and differentiation consequent to BAF complex inactivation (Narayanan et al., 2015; Nguyen et al., 2018). 

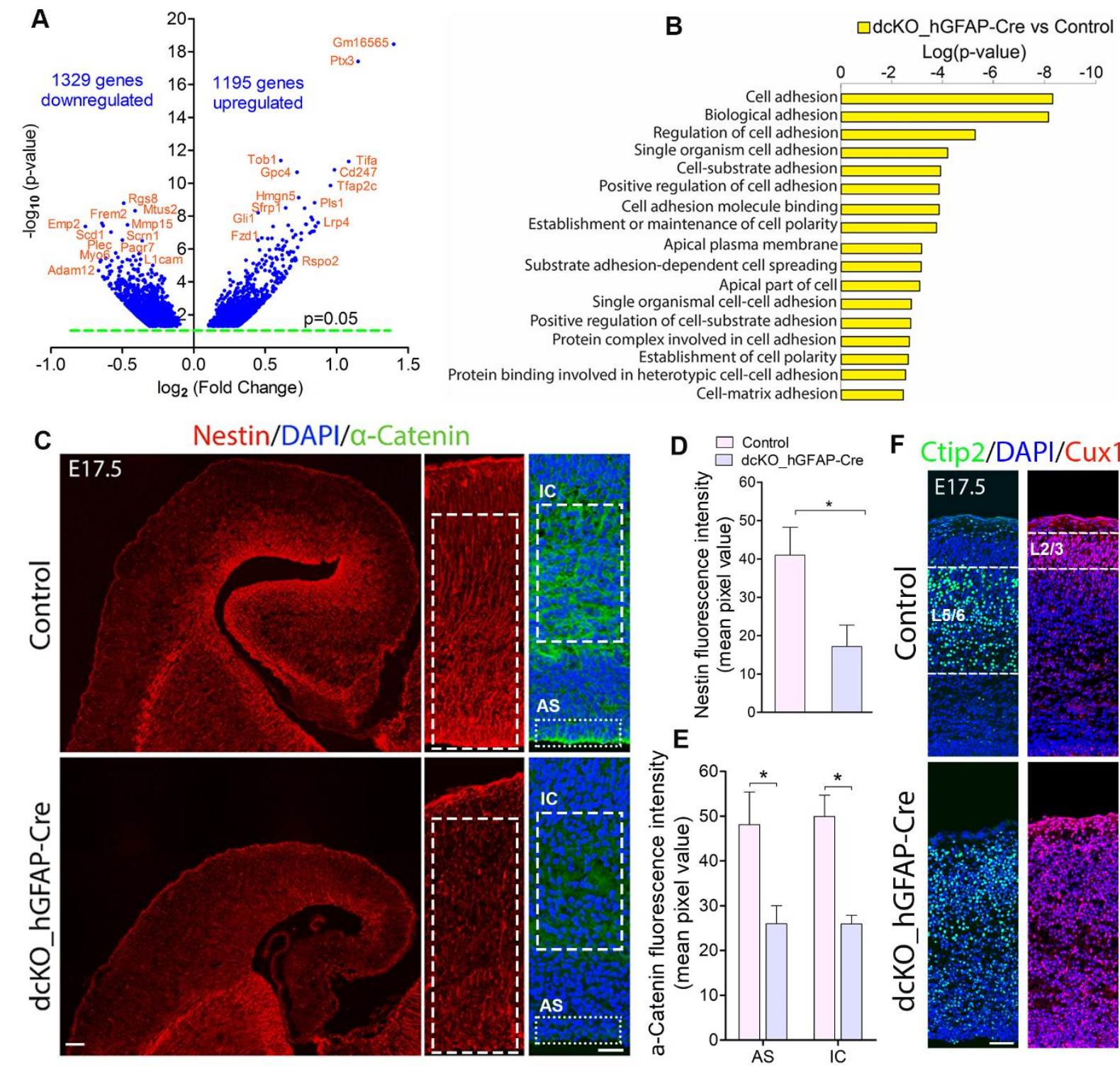

F Ctip2/DAPI/Cux1
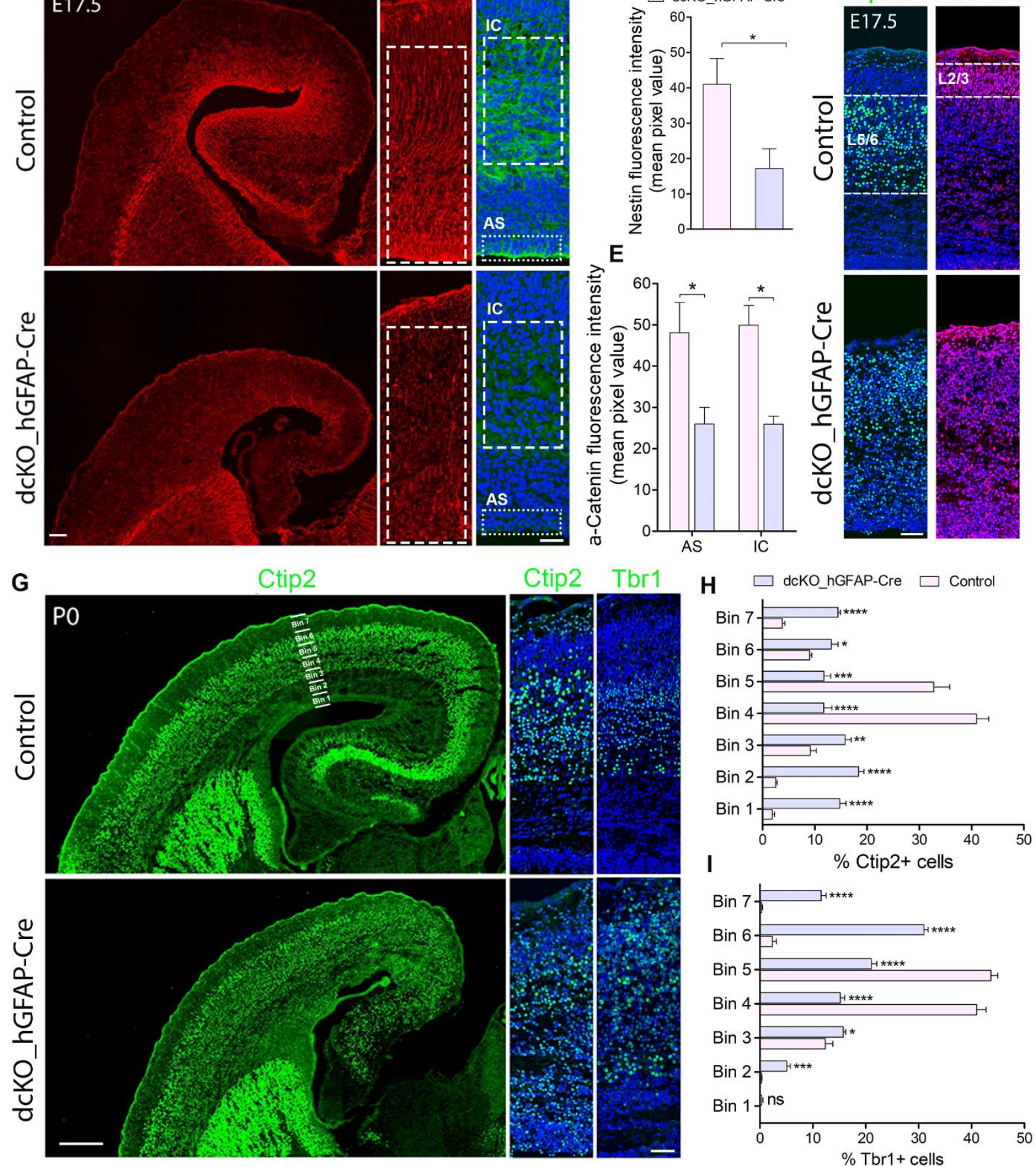
Figure 4.2 Cortical layer formation is improper in the absence of BAF complex due to loss of cell adhesion and glial fiber scaffolds: (A) Volcano plot showing genes downregulated and upregulated in the dcKO_hGFAP-Cre cortex. Examples of top altered genes are indicated. (B) Graph showing downregulation of selected gene category pathways mainly related to cell adhesion and polarity formation in the E17.5 dcKO_hGFAP-Cre cortex. (C) Sections of E17.5 control and dcKO_hGFAP-Cre cortex immunostained for the glial fiber protein Nestin and the cell adhesion protein $\alpha$-Catenin. White rectangular inserts represent respective cortical arears comparatively selected for quantifications. (D, E) Simple (D) and grouped (E) bar charts showing quantification of Nestin and $\alpha$-Catenin, respectively, in the E17.5 control and dcKO_hGFAP-Cre cortex. (F, G) Immunostaining with the lower and upper layer neuronal marker antibodies Ctip2 and Cux1, respectively, in the E17.5 (F) and P0 $(G)$ control and dcKO_hGFAP-Cre cortex. White broken lines are approximate upper and lower boundaries of the deep $(\mathrm{L} 5 / 6)$ and superficial (L2/3) cortical layers (F). Bins for neuronal distribution analysis are indicated in (G). $(\mathbf{H}, \mathbf{I})$ Bar charts showing quantitative distributions of deep layer neurons (Ctip2+ and Tbr1+) in the PO control and dcKO_hGFAP-Cre cortical wall. Where shown, sections are counterstained with DAPI (blue). Unpaired Student's $t$-test was used to test for statistical significance: ${ }^{*} p<0.05,{ }^{* *} p<0.005,{ }^{* * *} p$ $<0.0005,{ }^{* * *} \mathrm{p}<0.00005$; ns, not significant; $\mathrm{n}=3$; Scale bars: $=100 \mu \mathrm{m}$ and $50 \mu \mathrm{m}$ in overview and zoomed images, respectively. Results are presented as mean \pm SD. Abbreviations: Intracortical (IC), Apical surface (AS).

\subsubsection{Neuron-specific ablation of BAF complex caused delay in migration of upper layer neurons and perturbed cortical morphogenesis}

Even though compared with the dcKO_Emx1-Cre model the dcKO_hGFAP-Cre cortex is relatively more preserved and allowed us to more clearly examine the consequence of BAF complex deletion in neural progenitors (npBAF) on neuronal migration, we still have remarkable reduction in corticogenesis in the dcKO_hGFAP-Cre forebrain (Fig. 4.2G). Moreover, compared with lower L6 and L5 neurons (Fig. 2G), upper or superficial layer (L2-4) neuron generation is severely reduced in the postnatal dcKO_hGFAP-Cre cortex (Nguyen et al., 2018)). Therefore, to mainly restrict the phenotypic effect of BAF complex ablation to postmitotic neurons and preclude disturbance of neurogenesis, we resorted to the Nex-Cre line (Goebbels et al., 2006). This approach of manipulating the expression/function of BAF complex was expected to afford investigation of cortical neuronal migration with minimal interference in progenitor proliferation and differentiation. We generated the dcKO_Nex-Cre mouse line in which BAF155 and BAF170 (BAF complex) is deleted exclusively in the principal 
neurons generated in the cortex from E10.5 onwards (Fig. S1; (Goebbels et al., 2006)), although a few residual BAF subunits can be seen later in the mutant cortex (Fig. S4.2).

Characterization of the migration phenotype in the dcKO_Nex-Cre cortex was mainly done at early postnatal stages P1 and P6, as majority of projection neurons reach their final destination by then. Generally, the Nex-Cre-mediated BAF complex mutagenesis in the P1 dcKO_Nex-Cre cortex moderately impacted cortical size (Fig. 4.3A/B). To identify subtle migration defects, we performed fluorescence in situ hybridization (FISH) experiment in which the P1 dcKO_Nex-Cre and control cortex were riboprobed with the layer specific markers $T C, E t v, \operatorname{Ror} \beta$, and Ndnf which label the cortical layers $6,5,4,1$, respectively. Based on distribution of the aforementioned FISH probe signals, it was observed that the P1 dcKO_Nex-Cre cortical neurons were mis-distributed: making the cortical layers to appear amorphous as compared with the clearly delineated corresponding layers in the control cortex (Fig. 4.3A). Because the Ndnfmarked Layer 1 neurons are Cajal-Retzius cell which migrate tangentially into the cortex from other cortical areas like the hem (Bielle, Franck et al., 2005), they are presumably not affected by the Nex-Cre-mediated ablation of BAF complex expression; hence they displayed no disturbance in their laminar distribution in the P1 mutant cortex compared to control (Fig. 4.3A).

At the protein level, our immunohistochemical analysis showed that loss of BAF complex led to an impressive delay in the migration of Cux1+ upper layer neurons in the P1 dcKO_Nex-Cre cortex compared with control. Our bin analysis revealed significant number of Cux $1+$ neurons lagging in their radial migratory trajectories due to BAF complex inactivity (Fig. 4.3B/C). Interestingly, although the abnormal migration of upper (Cux1+) layer neurons in the P1 dcKO_Nex-Cre mildly persists in the P6 
dcKO_Nex-Cre cortex compared with control, the effect of the delayed migration greatly manifested as marked reduction in cortical size and structure of the P6 dcKO_Nex-Cre forebrain compared with control (Fig. S4.3A-E).

Consistent with the observed phenotype, we identified relevant abnormal alterations in gene expression levels in the P1 dcKO_Nex-Cre cortex. We found in our RNA-seq analysis that almost equal number of genes were downregulated (1167) and upregulated (1168) in the P1 dcKO_Nex-Cre cortex compare with control (Fig. 4.3D). Notably, some of the top downregulated genes include those that are associated with migration neurons (Fig. 4.3D). Also, our gene ontology analysis indicated downregulation of several cell differentiation-related pathways in the P1 dcKO_NexCre cortex (Fig. 4.3E). Specifically, we noticed that genes that are key for cytoskeletal remodeling needed for dendritic arborization and stability, and axonogenesis (Urbanska et al., 2008; Koleske, 2013) were downregulated in the BAF-deficient cortex (Fig. 4.3F).

To support the possible implication for defective neuronal differentiation in the dcKO_Nex-Cre cortical phenotype, we examined axonogenesis of upper layer neurons as a proxy for abnormal neuronal differentiation of dcKO_Nex-Cre neurons. Normally, it is the trailing process of migrating upper layer neuron that grows out as the axon to form of the corpus callosum. Strikingly, we found malformation (agenesis) of the corpus callosum as reveal by L1 immunostaining in rostral and caudal sections of the P6 dcKO_Nex-Cre cortex (Control vs dcKO_Nex-Cre in Fig. S4.3E). Thus, axonal formation and/or pathfinding is disturbed in neurons lacking BAF complex. Generally, neuronal differentiation is disturbed in the absence of BAF (SWI/SNF) complex or some of its subunits (Wu et al., 2007; John et al., 2012; Weinberg et al., 2013; Nguyen 
et al., 2018), and previous studies also showed that olfactory sensory neurons cannot form axons in the absence of BAF complex (Bachmann et al., 2016). Moreover, it is a possible phenomenon that perturbation of neuronal migration, especially of upper layer neurons, can cause loss of cortical interhemispheric connection (Boitard et al., 2015; Bocchi et al., 2017).

Altogether, we have shown that exclusive deletion of BAF complex in postmitotic neurons perturbs neuronal differentiation program that can lead to improper formation of neuronal processes necessary for oriented-neuronal migration and development of the mammalian cortical structure.
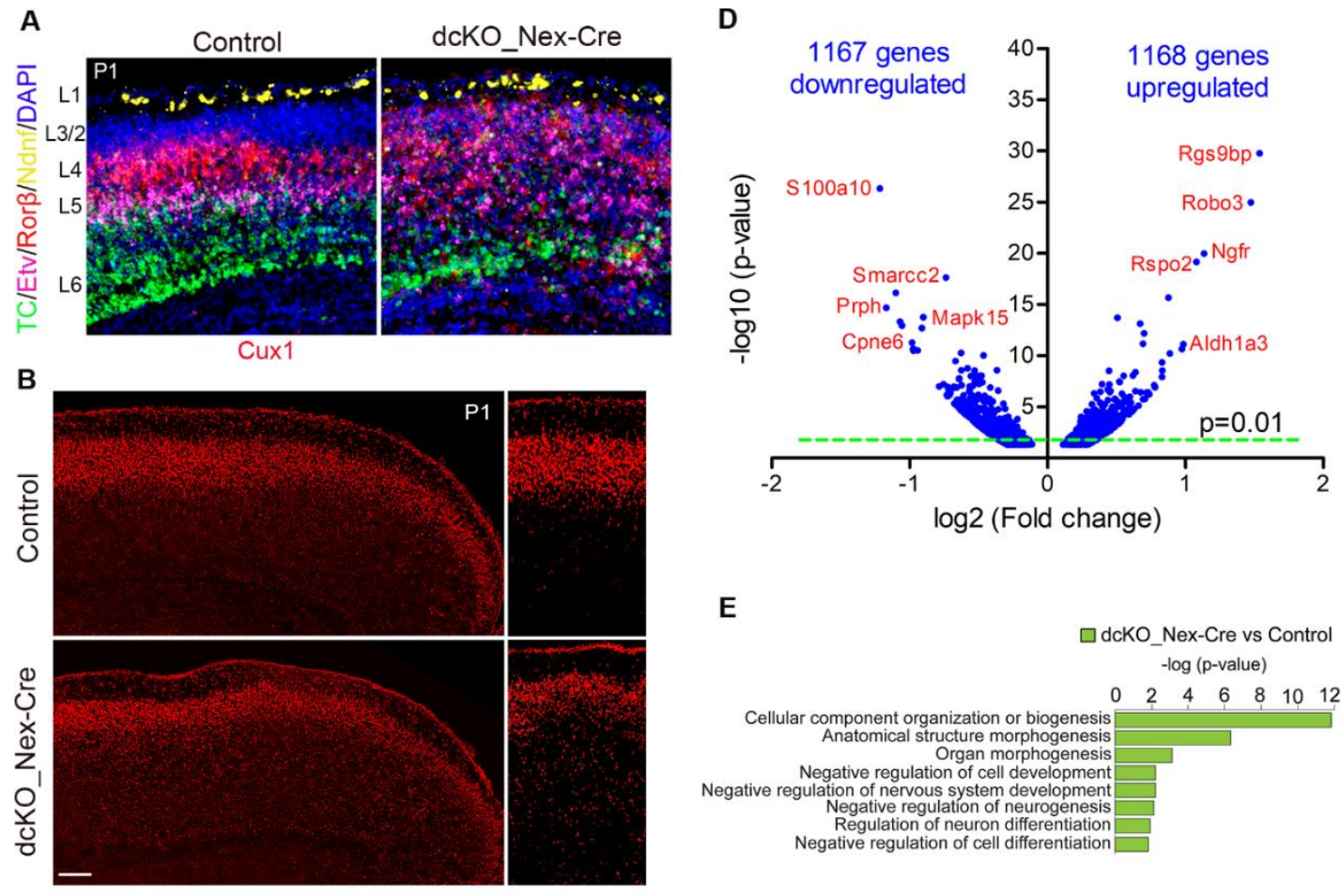

E
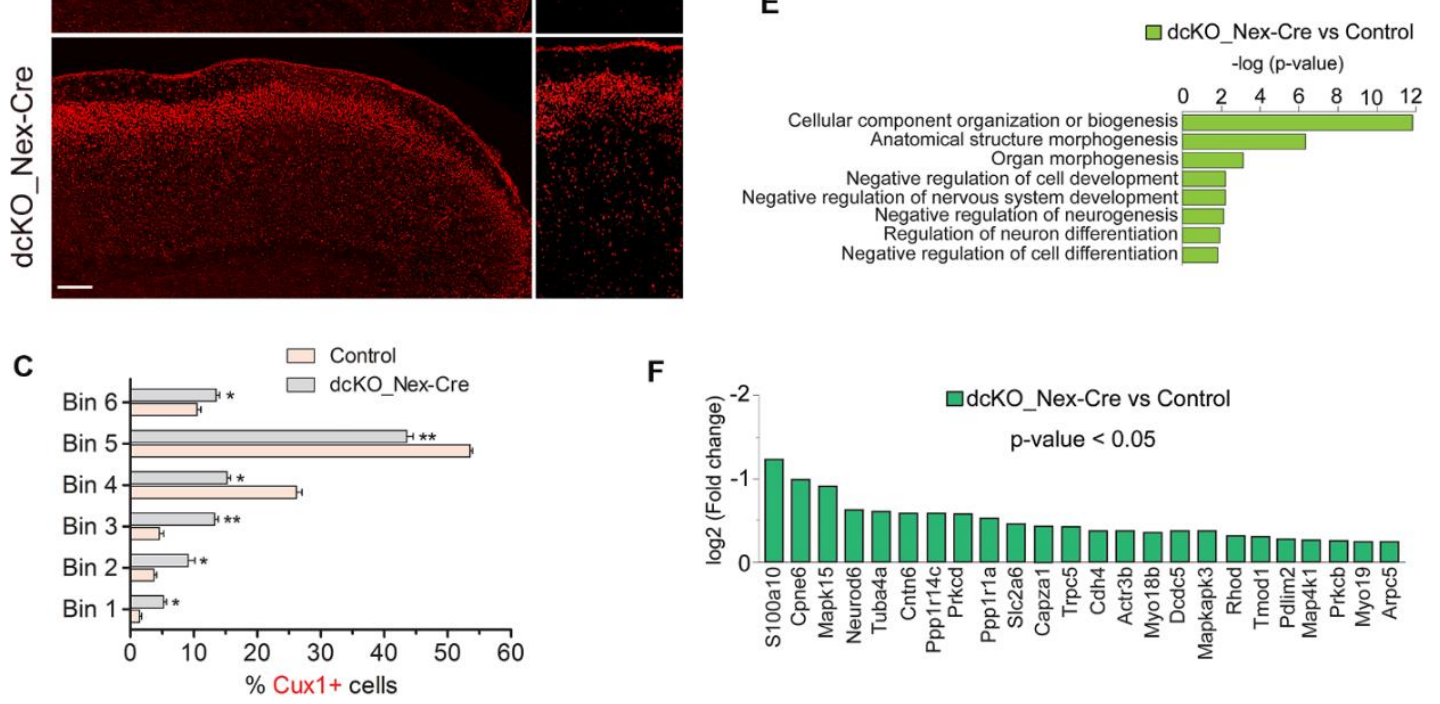
Figure 4.3 Neuron-specific deletion of BAF complex alters neuronal differentiation programs leading to defective neuronal migration: $(\mathbf{A})$ Micrographs showing fluorescence in situ hybridization in the P1 control and dcKO_Nex-Cre cortex stained with the layer specific RNA probes TC, Etv, Ror $\beta$, and Ndnf to reveal cortical layers 6, 5, 4, and 1, respectively. (B) Micrographs showing immunostaining of the P1 control and dcKO_Nex-Cre cortex with Cux 1 antibodies to mainly mark layer $2 / 3$ neurons. (C) Bar chart showing quantitative distribution of Cux1+ neurons in the P1 control and dcKO_hGFAP-Cre cortical wall. (D) Volcano plot show genes downregulated and upregulated in the P1 dcKO_Nex-Cre cortex compared with control. Examples of top altered genes are highlighted in red. (E) Gene ontology analysis showing differentiation-related pathways altered in the P1 dcKO_Nex-Cre cortex. (F) Chart showing specific neuronal differentiation- and polarization-related genes with reduced expression in the P1 dcKO_Nex-Cre cortex. Where shown, sections are counterstained with DAPI (blue). Unpaired Student's $t$-test was used to test for statistical significance: ${ }^{*} p<0.05,{ }^{* *} p<0.005 ; n=6$; Scale bar $=100$ $\mu \mathrm{m}$. Results are presented as mean \pm SD.

\subsubsection{Knockdown of BAF155 and BAF170 in cortical progenitors revealed impairment of oriented-neuronal migration and neurite formation}

In order to refine the presentation of cortical neuron migration phenotype due to loss of BAF complex so as to identify any alteration of the cellular dynamics involved, we employed in utero electroporation technique (Fig. 4.4A). This focal ablation strategy afforded sparse loss of the BAF complex in selected cortical neurons, thus making the resultant migration phenotype more conspicuous. To achieve this, we electroporated the mouse cortex double floxed for BAF155 and BAF170 with a control plasmids pCIG2-ires-eGFP (CAG-eGFP/GFP-only) or pCIG2-Cre-ires-eGFP (CAG-Cre-eGFP) plasmids into the E14.5 cortex (Fig. 4.4A). Because the CAG-Cre is active in all cell types, we essentially mimicked loss of BAF complex under the Emx1- or hGFAP-Cre promoters as achieved in the dcKO_Emx1-Cre and dcKO_hGFAP-Cre cortical models (Fig. 4.1, Fig. 4.2). The electroporated embryos were allowed to develop until E17.5 and the cortical tissues collected for immunohistological analyses.

By means of GFP immunostaining, we were able to track the progress of transfected migrating cortical neurons from the Pax6-marked VZ to the CP. Interestingly, we found that the GFP-only transfected neurons migrated in discernable radial profiles, whereas the CAG_Cre+GFP positive neurons displayed apparent lateral dispersion and less 
obvious radial trajectories (Fig. 4.4B). Also, we observed formation and fasciculation of the axons of the GFP-only transfected neurons, whereas the CAG_Cre+GFP transfected neurons failed to show noticeable axonogenesis; signifing possible differentiation incompetence in the neurons lacking BAF155 and BAF170 (Fig. 4.4B). This can be linked to abnormal RG cell fiber-neuron contact or adhesion which can disrupt axon formation and orientation during locomotion (Xu et al., 2015).

At higher magnification, it was evident that glial fiber-dependent (radial) migration of cortical neurons was disrupted following loss of BAF155 and BAF170. Compared with control, the BAF complex mutant (i.e. CAG_Cre-treated) neurons, presumably multipolar neurons, appeared to have accumulated in the lower regions of the cortical wall namely the Pax6-labeled VZ, SVZ, and IZ, making the upper and lower aspects of the CP less populated with successfully migrated and/or properly migrating neurons (Fig. 4.4C/D). We identified that the cortical neurons without functional BAF complex were mis-oriented with respect to their normal radial alignment and also exhibited abnormal polarities. This was revealed in our immunohistological examination of the Golgi apparatus localization and leading process length of the actively migrating neuron in the middle and upper IZ (Fig. 4.4E/F). The BAF complex-ablated bipolar neuron has its GM130+ Golgi located close to the nucleus and with reduced estimated leading process length compared with control neurons in the upper IZ ( $p$-value $=$ 0.0032, Mann Whitney Test; Fig. 4.4F-H). This likely underpined the decreased proportion of migrating neurons facing the pia, a proxy for proper radial migration, in mutants (CAG_Cre+GFP-transfected neurons) compared with control (GFP-onlytransfected neurons) (Fig. 4.4J) and gives reason to the abnormal radial migration seen in the BAF complex knockdown condition (Fig. 4.4C/D). 
In further support of our assertion of defective directed-neuronal migration when BAF complex is inactivated in neurons, we observed reduced engagement of Nestin+ glial fibers by the CAG_Cre+GFP positive migrating neurons compared with the GFP-only positive neurons in the upper IZ and lower CP (Fig. 4.4I). In other words, based on neuron-glial fiber proximation (i.e. GFP and Nestin signal colocalization), whereas more than $80 \%$ of the control migrating neurons depend on or use the glial fiber scaffolds to radially migrate, far less proportion (16\%) of CAG_Cre+GFP positive neurons seem to use glial fibers for migration (Fig. 4.4I/K). Moreover, given the role of adhesion molecules in radial migration (Solecki, 2012), we inferred from the loss of cell adhesion in the developing cortex due to deletion of BAF155 and BAF170 (Fig. 4.1BF; Fig. 4.2A-E) that it is possible the CAG_Cre+GFP positive neurons improperly attach to glial fibers or not at all because of loss of glial fiber and adhesion proteins leading to possible abolishment of normal radial migration (Fig. 4.4I/L).

In all, the reduced use of glial fibers for migration by the BAF complex-ablated cortical neurons and their dysregulated Golgi-dependent polarization and transformation, may have contributed to the loss of radial orientation. These deficiencies may have implications for possible sluggish neuronal motility and mis-guidance leading to stalling of neuronal trekking in the developing cortex (Fig. 4.4C/D/L). 
A
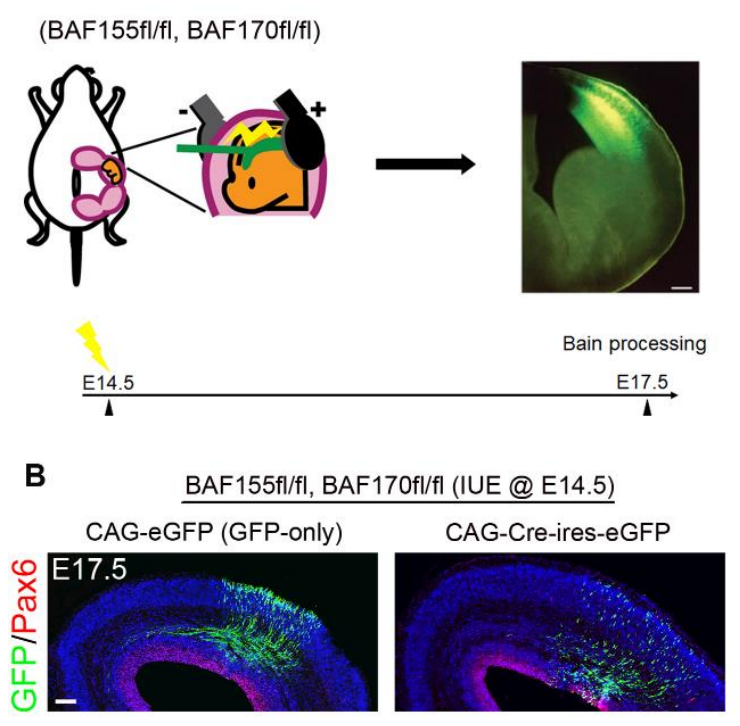

C BAF155fl/fl, BAF170fl/fl (IUE @ E14.5)

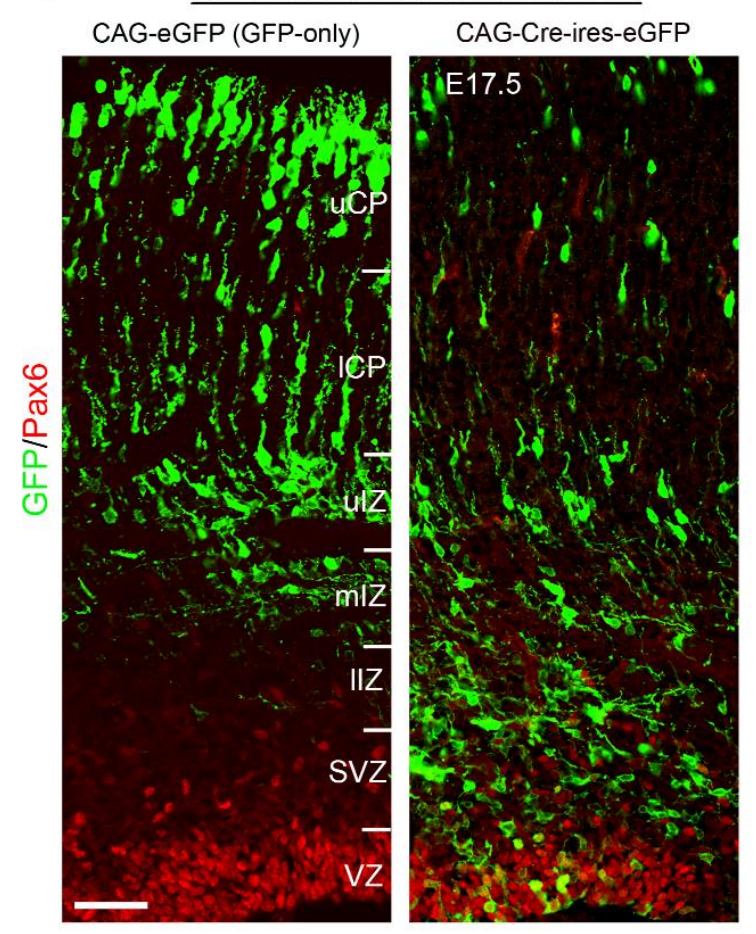

D

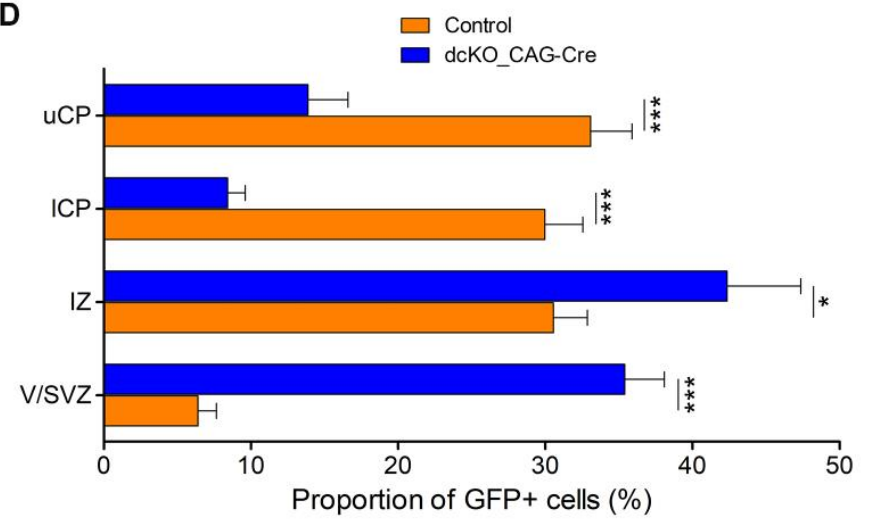

E BAF155fl/fl, BAF170fl/fl (IUE @ E14.5)
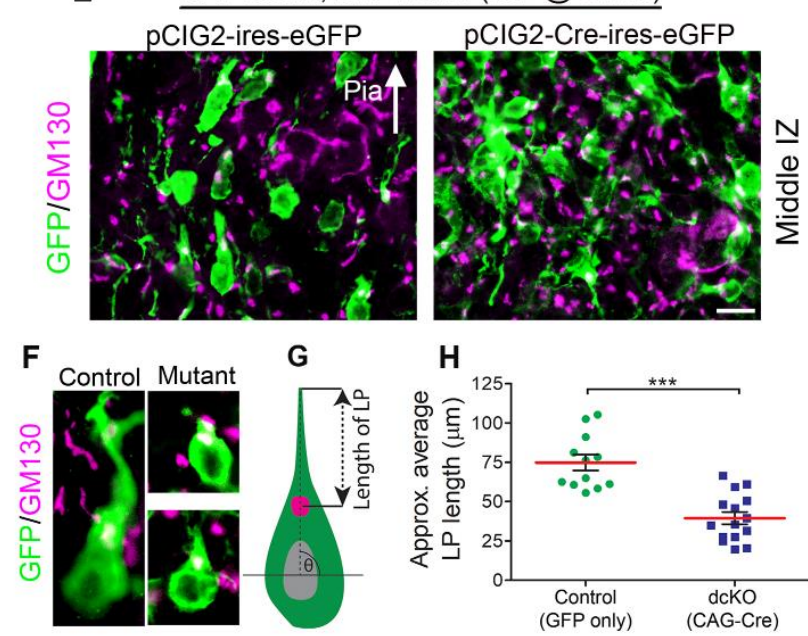

G $\quad H$

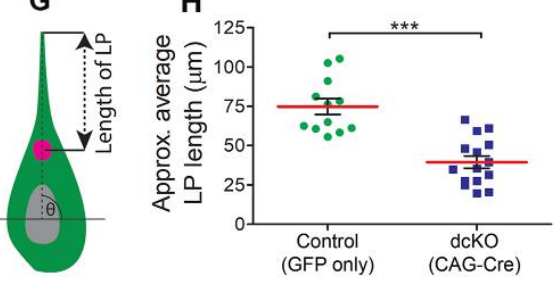

I $\frac{\text { BAF155fl/fl, BAF170fl/fl (IUE @ E14.5) }}{\text { CAG-eGFP CAG-Cre-ires-eGFP }}$
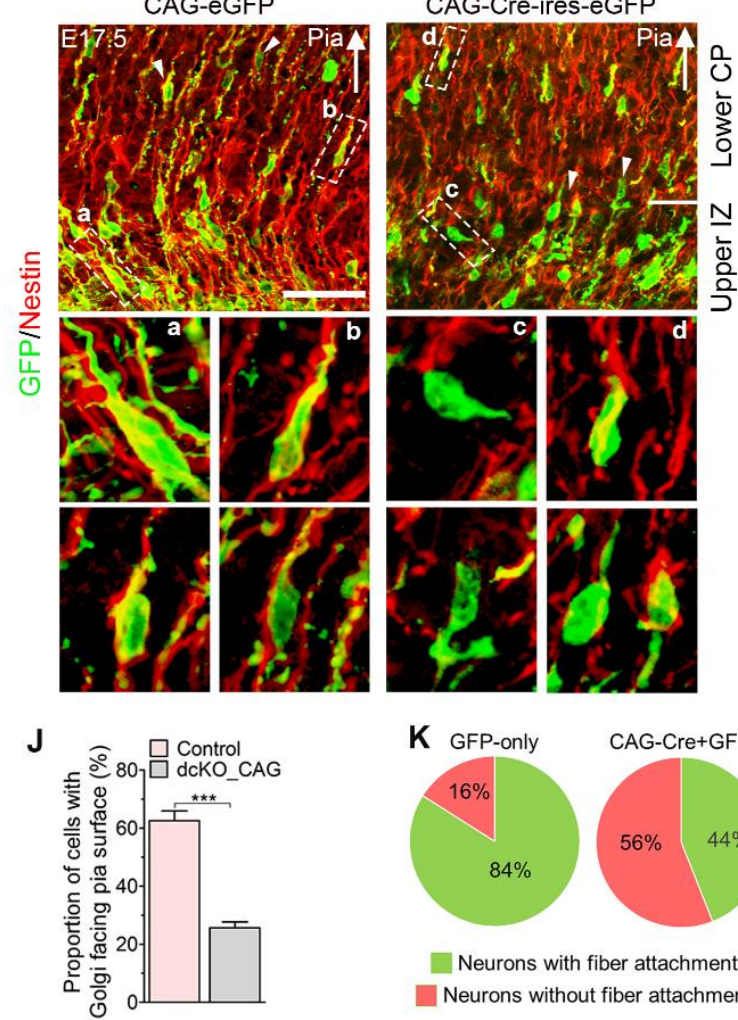

K GFP-only CAG-Cre+GFP

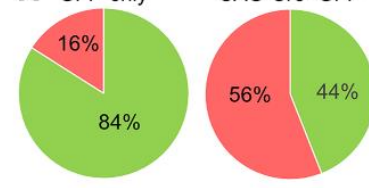

Neurons with fiber attachment Neurons without fiber attachment

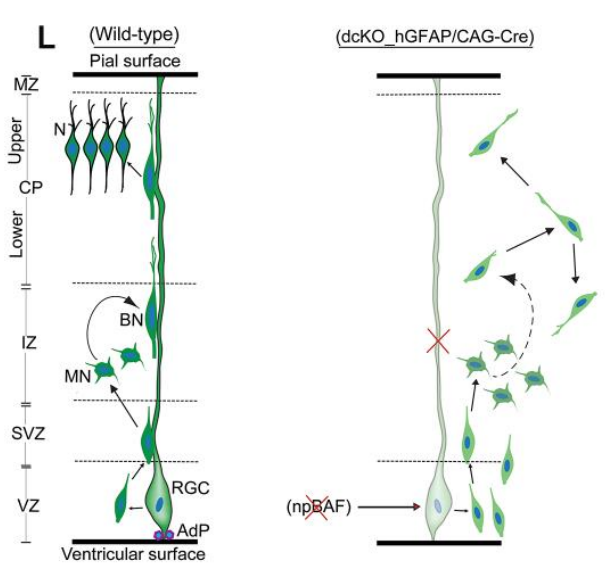


Figure 4.4 Migrating neurons without BAF complex exhibit abnormal polarity and radial misguidance: (A) Illustration of in utero electroporation technique used to achieve knockdown of BAF155 and BAF170. It shows a representative embryo in the uterine horn double floxed for BAF155 and BAF170 (BAF155fl/fl, BAF170fl/fl) that is being injected in the brain with plasmid of interest and electroporated at E14.5, and the brain tissue harvested at E17.5. (B) Immunohistochemical micrographs showing overview of Pax6 (to mark the germinal zones) and GFP staining in the E17.5 cortex (BAF155fl/fl, BAF170fl/fl) electroporated with control (GFP-only) or CAG-Cre+GFP plasmids. (C) Micrographs showing the electroporated cortical areas at higher magnification with Pax6 and GFP immunostaining in the control and CAG-Cre+GFP plasmid-injected E17.5 cortex. Approximate cortical regions are shown. (D) Bar chart showing distribution of GFP+ neurons in the various cortical regions in the E17.5 control and CAG-Cre+GFP plasmid-injected E17.5 cortex. (E) Images showing the E17.5 control and mutant cortical middle intermediate zone with GFP and GM130 staining which marks the Golgi apparatus. (F-H) Micrographs with GFP and GM130 staining (F) and illustration (G) showing estimation of the leading process (LP) length of control and BAF complex mutant cortical neurons in the lower cortical plate graphically compared in (H). (I) Images of the E17.5 control and mutant upper intermediate zone and lower cortical plate immunostained with GFP and Nestin antibodies to reveal neuron-glial fiber colocalization. Inserts ( $a, b, c$ and d) and arrow heads indicate examples of neurons migration with or without (abnormally with) glial fiber guide. (J) Bar chart showing the proportion of GFP+ neurons with their Golgi-leading process axis oriented towards the pia in the E17.5 GFP-only and CAGCre+GFP-electroporated cortical upper intermediate zone. (K) Pie chart depicting the quantification of the proportion of GFP+ control and BAF complex mutant neurons with or without glial fiber guide in the upper intermediate zone and lower cortical plate. (L) Graphical summary of the abnormal neuronal migration due to BAF complex deletion in neural progenitors as compared with control. Solid curved arrow indicates normal transition and broken curved arrow denotes truncation/protraction of transition. Where shown, sections are counterstained with DAPI (blue). Vertical arrows point in the direction of the pia. Red cross denotes deletion or loss. Unpaired Student's $t$-test was used to test for statistical significance: ${ }^{*} p<0.05,{ }^{* * *} p<0.0005 ; n=4$, Selected 25 and 17 control and mutant GFP+ ulZ neurons for leading process length estimation, respectively; Scale bars: $=100 \mu \mathrm{m}$ and $50 \mu \mathrm{m}$ in overview and zoomed images, respectively. Results are presented as mean \pm SD. Abbreviations: Ventricular zone (VZ), Subventricular zone (SVZ), (lower/middle/upper) Intermediate zone (I/m/ulZ), lower/upper cortical plate (I/UCP), Marginal zone (MZ), Neuron (N), Bipolar neuron (BN), Multipolar neuron (MN), Radial glial cell (RGC), Adhesion protein (AdP), Neural progenitor BAF complex (npBAF).

\subsubsection{BAF complex-deficient migrating cortical neurons display defective}

\section{multipolar-to-bipolar transition leading to abnormal migration}

Applying the same logic and advantage of using the dcKO_Nex-Cre line over the dcKO_Emx1- and hGFAP-Cre lines, we neuron-specifically knocked-down BAF155 and BAF170 in the E14.5 cortex using a NeuroD-Cre-ires-GFP plasmid in another in utero electroporation experiment. Thus, the NeuroD-Cre-mediated inactivation of BAF complex in nascent cortical neurons increased the specificity of the related effect on radial migration of neuron as compared with the CAG-Cre model.

Examination of GFP immunostaining in two sets of E17.5 cortices (double floxed for BAF155/BAF170) electroporated with control (NeuroD-GFP) and NeuroD-Cre-ires- 
GFP plasmids at E14.5 revealed marked disruption of radial neuronal migration (Fig. 4.5A). Although the Pax6-stained VZ in the cortex transfected with NeuroD-GFP or NeuroD-Cre-ires-GFP plasmids is devoid of GFP+ cells, the IZ and lower (I) CP of the NeuroD-Cre-ires-GFP electroporated cortex is filled with more GFP + cells compared with the control cortex (Fig. 4.5A/B). However, as an indication of successful radial migration, the control upper $(\mathrm{u}) \mathrm{CP}$ is seen to be filled with significantly more GFP+ cells as compared with the uCP of NeuroD-Cre-ires-GFP treated cortex (Fig. 4.5A/B).

One of the critical cellular processes during radial migration of cortical neuron is the transient transformation from multipolar to bipolar morphology which occurs in the upper IZ (Nadarajah et al., 2001; Noctor et al., 2004). The function of the multipolar phase is not clearly known, albeit some believe directional cues are collected by the many temporary neurites of the neurons at this stage (Mizutani, 2018). The bipolar structure on the other hand is suited for the glial fiber attachment and subsequent active locomotion to the CP (Nadarajah et al., 2001; Noctor et al., 2004). This information evoked our interest in examining the morphological integrity of migrating neurons lacking BAF complex. We sampled and grouped the diverse forms of migrating neurons in electroporated regions of the cortical wall into three morphological categories: unipolar/bipolar, multipolar, and non-polar (undefined) neurons (Fig. 4.5C). Our statistical quantification revealed significantly less proportion of bipolar or unipolar neurons in the NeuroD-Cre-ires-GFP electroporated cortical area compared with the NeuroD-GFP electroporated cortical area. However, we found more multipolar neurons in the NeuroD-Cre-ires-GFP electroporated cortex compared with the cortical area electroporated with NeuroD-GFP plasmid (Fig. 4.5D). Strikingly, we also found more so-called nonpolar neurons in the NeuroD-Cre-ires-GFP treated cortex, particularly in 
the $\mathrm{CP}$, as compared with that in the control cortex. Such nonpolar neurons displayed undefined structural forms (Fig 4.5A/D).

Based on these observations and as schematized in Figure 5D, our findings implicate the BAF complex in the regulation of multipolar-to-bipolar transition of cortical neurons during radial migration. As such, in the event of BAF complex dysfunction (NeuroDCre-ires-GFP condition), this neuronal morphology conversion is hindered, leading to accumulation of multipolar neurons in the $\mathrm{IZ}$ at the expenses of bipolar neurons in the $\mathrm{CP}$ (Fig 4.5A/D/E). Moreover, even if it occurs, the morphologic transformation of the NeuroD-Cre-ires-GFP plasmid-transfected neurons is abnormal and resulted in the formation of non-polar neurons (Fig. 5A/C/D) or aberrantly polar neurons as displayed by neurons with CAG-Cre-mdiated BAF complex deletion (Fig. 4.4F/H). Thus, it is conceivable that the NeuroD-Cre-ires-GFP electroporated neurons undergo unconventional or complicated migration other than the desirable radial (glial-guided) migration that is prerequisite for correct laminar formation in the developing cortex (Fig. $4.5 \mathrm{E})$.

Together, we identified the BAF complex to also regulate the glial fiber-independent process of multipolar-to-bipolar neuronal morphology transition which is a ratedetermining phase in the process of radial migration permissive for correct establishment of the various cortical layers, especially upper layers, during forebrain development. 
A

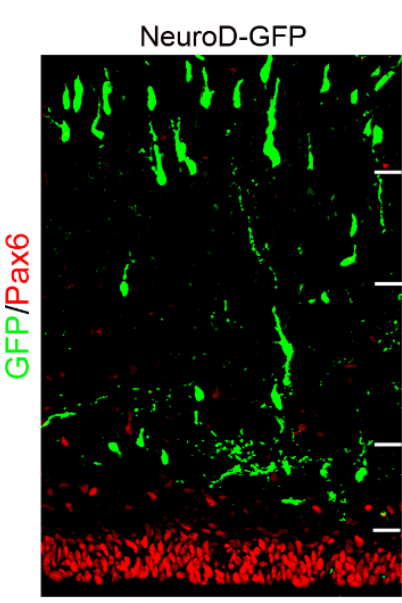

B

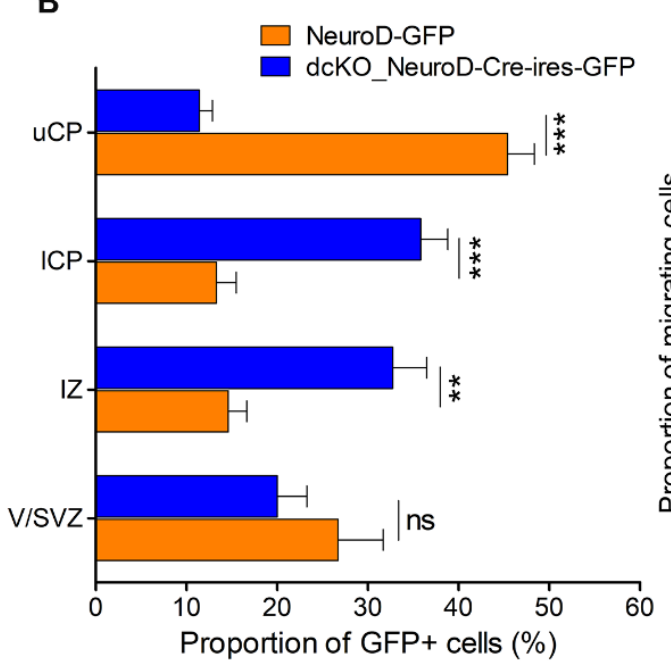

C NeuroD-Cre-ires-GFP

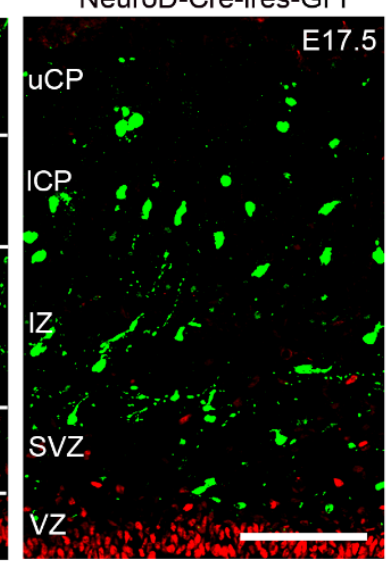

Neuronal morphology categorisation
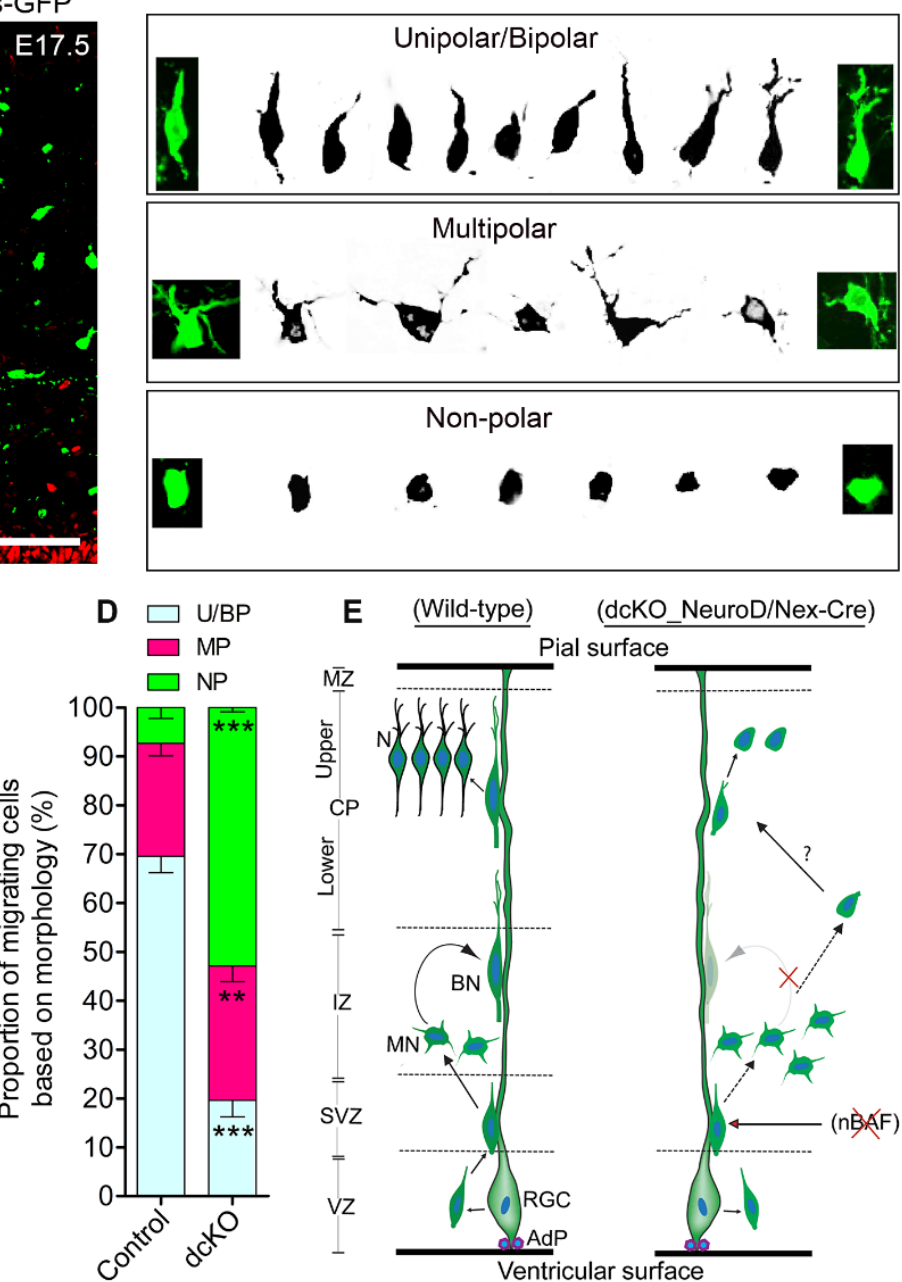

Figure 4.5 BAF complex regulates multipolar-to-bipolar neuronal morphology transition during radial migration: (A) Micrographs showing Pax6 and GFP immunostaining in the E17.5 cortex (BAF155fl/fl, BAF170fl/fl) electroporated with control (NeuroD-GFP) or Cre (NeuroD-Cre-ires-GFP) plasmids. Approximate cortical regions are shown. (B) Bar chart showing percentage distribution of GFP+ neurons in the various cortical regions in the E17.5 control and NeuroD-Cre-ires-GFP plasmidinjected E17.5 cortex. (C) Images showing representative morphological groupings of the migrating neurons with or without BAF complex in the E17.5 control or mutant cortex. (D) Composite bar graph showing the quantitative proportions of the various categories of neuronal morphologies in the E17.5 cortical areas electroporated with control or Cre plasmids. (E) Graphical summary of the abnormal neuronal migration due to BAF complex deletion in neurons as compared with control. Solid curved arrow indicates normal transition, broken straight arrows denote abnormal transition, and red crossed lines indicate blockage. Unpaired Student's $t$-test was used to test for statistical significance: ${ }^{* *} p<0.005$, ${ }^{* * *} p<0.0005$; ns, not significant; $n=4$; Scale bar: $=50 \mu \mathrm{m}$. Results are presented as mean \pm SD. Abbreviations: Ventricular zone (VZ), Subventricular zone (SVZ), Intermediate zone (IZ), Lower/upper cortical plate (I/UCP), Marginal zone (MZ), Neuron (N), Unipolar/Bipolar neuron (U/BN), Multipolar neuron (MN), Radial glial cell (RGC), Adhesion protein (AdP), Neuronal BAF complex (nBAF). 


\subsubsection{BAF complex may repress WNT signaling to permit neuronal migration and cortical lamination}

In order to identify possible molecular factors or mechanism(s) that mediate BAF complex influence on radial migration of cortical neurons, we screened the results of our molecular assays in the BAF complex mutant and control developing cortex. Some major signaling pathways were identified to be upregulated in the BAF155 and BAF 170 dcKO developing cortex namely: the Bone morphogenetic proteins (BMP), NOTCH, Sonic hedgehog (SHH) and the Wingless/Int (WNT) pathways (summarized in Fig. 6A). Interestingly, all the aforementioned signaling pathways have been implicated in neuronal migration regulation during brain development (Hashimoto-Torii et al., 2008; Boitard et al., 2015; Bocchi et al., 2017; Martinez-Chavez et al., 2018; Saxena et al., 2018). In particular, dynamic regulation of WNT signaling was reported to be necessary for multipolar-to-bipolar morphology transition during radial migration of cortical neurons (Boitard et al., 2015). Of note, unlike the other signaling pathways, the WNT pathway was upregulated in all our dcKO models (asterisked in Fig. 6A). This made us to consider the WNT pathway as a potential molecular candidate for our rescue experiment.

Following a gene ontology analysis in the E17.5 dcKO_hGFAP-Cre cortex, we found many WNT pathways to be upregulated as compared with control ((Nguyen et al., 2018)). We then proceeded with designing a generalizable rescue experimental paradigm that can plausibly be extrapolated to the other BAF complex mutant cortical models with increased WNT activity generated in the study. Given that the dcKO_Emx1-Cre cortex was unfit for clearly visualizing neuronal migration dynamics because of massive cortical atrophy (Fig. $4.1 \mathrm{~A}$ ), and the migration phenotype in the 
dcKO_Nex-Cre cortex is milder at later postnatal stages (Fig. S4.3E), we choose the dcKO_hGFAP-Cre cortex for our rescue experiment.

To perform the rescue experiment, we employed the in vivo chemical inhibition approach to downregulate (knockdown) the upregulated WNT signaling in the developing dcKO_hGFAP-Cre cortex. This was achieved with the chemical ICG-001, a small molecule capable of interfering with CREB-binding protein (CBP) and $\beta$-catenin complexing during WNT signaling, leading to inhibition of the pathway and its related downstream molecular and cellular effects (Emami et al., 2004; Teo et al., 2005; Wiese et al., 2017). Mice pregnant with dcKO_hGFAP-Cre embryos were intraperitoneally injected with daily dose of ICG-001 from E11.5 to E16.5 and the brains were harvested for histological analysis at E17.5 (Fig. 4.6B). Immunostaining with Ctip2 and Cux1 antibodies revealed partial rescue of radial neuronal migration and formation of deep and superficial cortical layers in the WNT inhibitor (WNTi)-treated dcKO_hGFAP-Cre cortex compared with the dcKO_hGFAP-Cre without WNTi pharmacological treatment (Fig. 4.6C/D). Our bin analysis showed that the apparent crowding of Ctip2+ lower layer neurons and Cux1+ upper layer neurons in the dcKO_hGFAP-Cre lower cortical wall region significantly resolved due to migration of many of such neurons to populated their designated laminae in the CP upon downregulation of WNT signaling (Fig. 4.6C-F). We also observed more preserved RG fibers and increased expression of apical and intracortical $\alpha$-Catenin (cell adhesion) in the WNTi-treated dcKO_hGFAPCre cortex compared with dcKO_hGFAP-Cre (Fig. 4.6G-J). Probably, WNT inhibition in the dcKO_hGFAP-Cre cortex afforded maintenance of glial fibers and cell adhesion, and normalization of multipolar-to-bipolar neuronal morphology transition which promoted correct radial migration (Fig. 4.7). 
In all, we show that the BAF complex is a critical regulator of the glial fiber-dependent and independent aspects of radial migration of cortical neurons through modulation of WNT signaling activity in the developing mouse forebrain to allow proper lamination during cortical layer patterning.

A

Major signaling pathways altered in BAF mutants

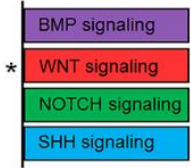

* dcKO (Emx1-/hGFAP-/Nex-Cre)

dcKO (Emx1-/hGFAP-Cre)

dcKo (Emx1-/hGFAP-Cre)
B

Chemical inhibition
approach with ICG-001

ICG-001

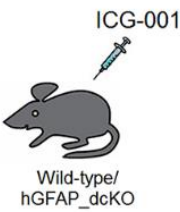

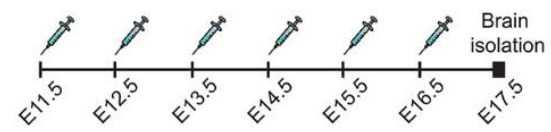
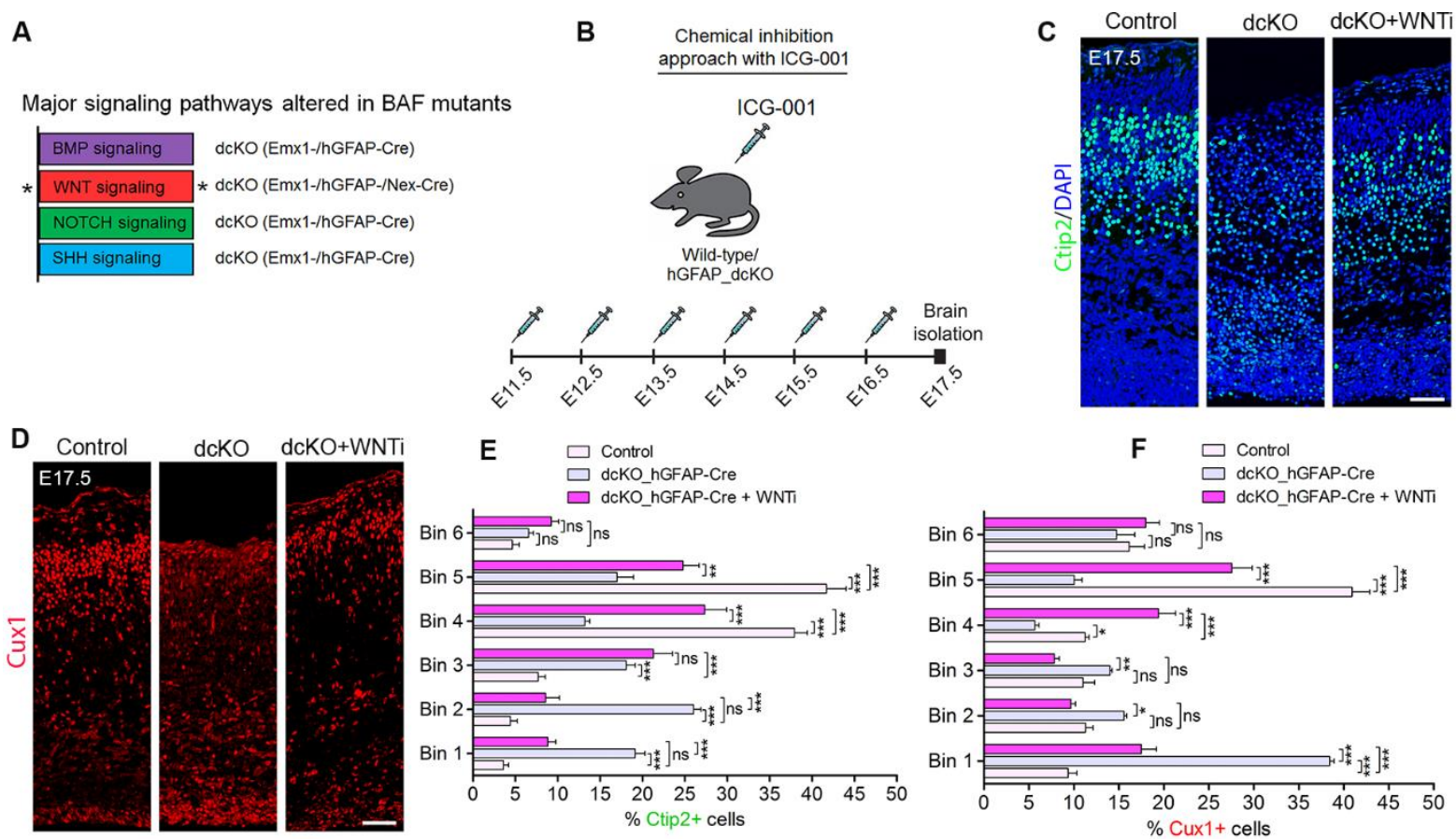

E $\square$ Control
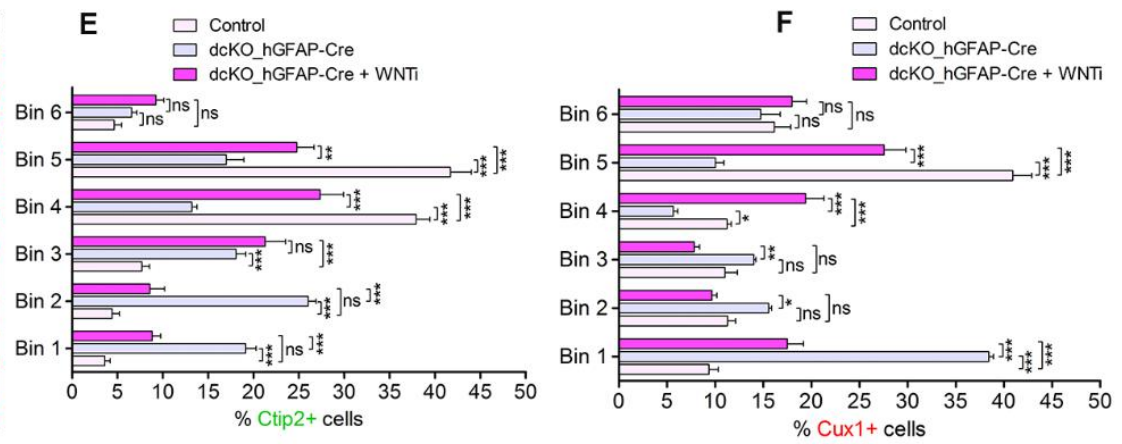

\section{G Control}
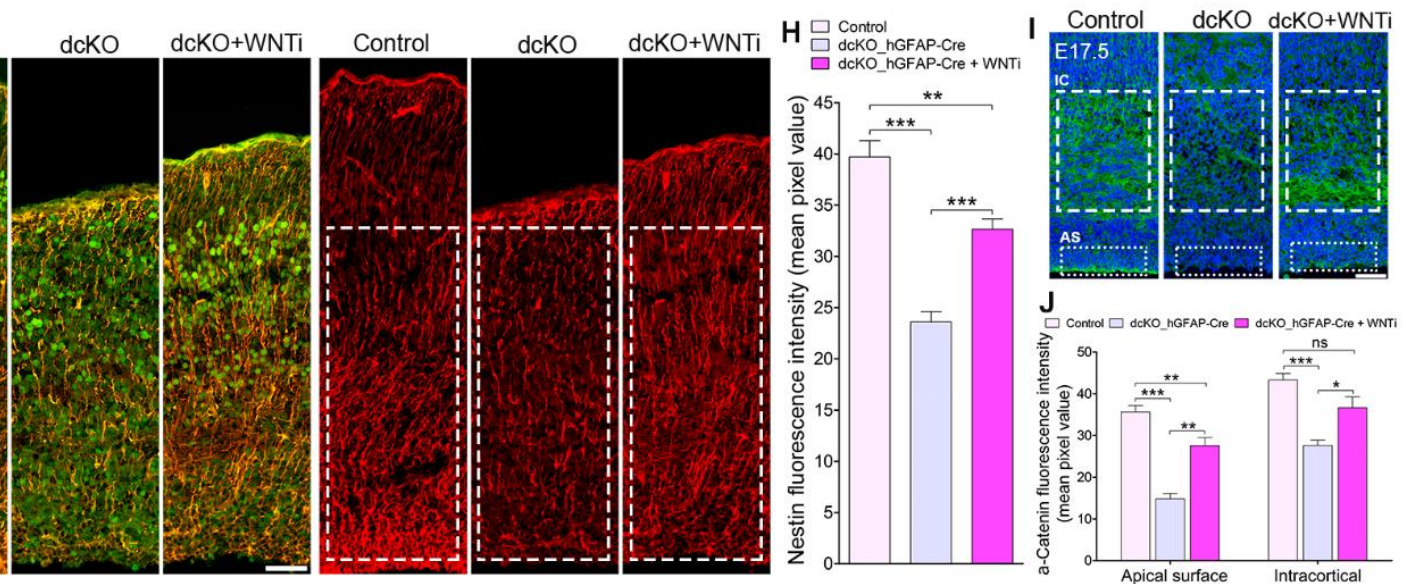

Figure 4.6 BAF complex modulates WNT signaling to drive cortical lamination: (A) Illustration showing the major signaling pathways upregulated in the developing cortex due to loss of BAF complex. Asterisks are used to highlight the WNT signaling pathway being the one upregulated in all our conditional knockout cortical models. (B) Illustration of the scheduled intraperitoneal injection of pregnant mouse carrying dcKO_hGFAP-Cre embryos with the WNT inhibitor (WNTi) ICG001. (C, D) Immunomicrographs showing Ctip2+ (C) and Cux1+ (D) neuronal distribution partially rescued in the E17.5 dcKO_hGFAP-Cre cortex following WNTi treatment compared with the dcKO_hGFAP-Cre without ICG001 treatment and control cortex. (E, F) Bar graphs showing quantitative bin analyses of the Ctip2+ (E) and Cux1+ (F) neuronal distribution rescue in the E17.5 dcKO_hGFAP-Cre and WNT 
inhibitor-treated dcKO_hGFAP-Cre cortex compared with control. (G) Immunomicrographs showing Nestin staining with and without Ctip2+ neuronal distribution background in the E17.5 control, dcKO hGFAP-Cre and dcKO hGFAP-Cre with WNT inhibitor treatment cortex. (H) Bar chart indicating quantification of Nestin + glial fiber partial rescue in the WNT inhibitor-treated dcKO_hGFAP-Cre cortex compared with the dcKO_hGFAP-Cre and control cortex. (I) Images showing intracortical (IC) and apical surface (AS) expression of $\alpha$-Catenin in the E17.5 control, dcKO_hGFAP-Cre and dcKO_hGFAP-Cre with WNT inhibitor treatment cortex. (J) Graphical representation of the quantification of $\alpha$-Catenin expression following WNT inhibition in the dcKO_hGFAP-Cre cortex compared with dcKO_hGFAP-Cre without WNT inhibitor treatment and control cortex. White rectangular inserts represent respective cortical arears comparatively selected for quantifications. One-way ANOVA followed by Bonferroni posthoc analysis and unpaired Student's $t$-test were used to test for statistical significance: ${ }^{*} p<0.05,{ }^{* *} p<$ $0.005,{ }^{* \star} \mathrm{p}<0.0005$; ns, not significant; $\mathrm{n}=4-6$; Scale bar: $=50 \mu \mathrm{m}$. Results are presented as mean \pm SD.

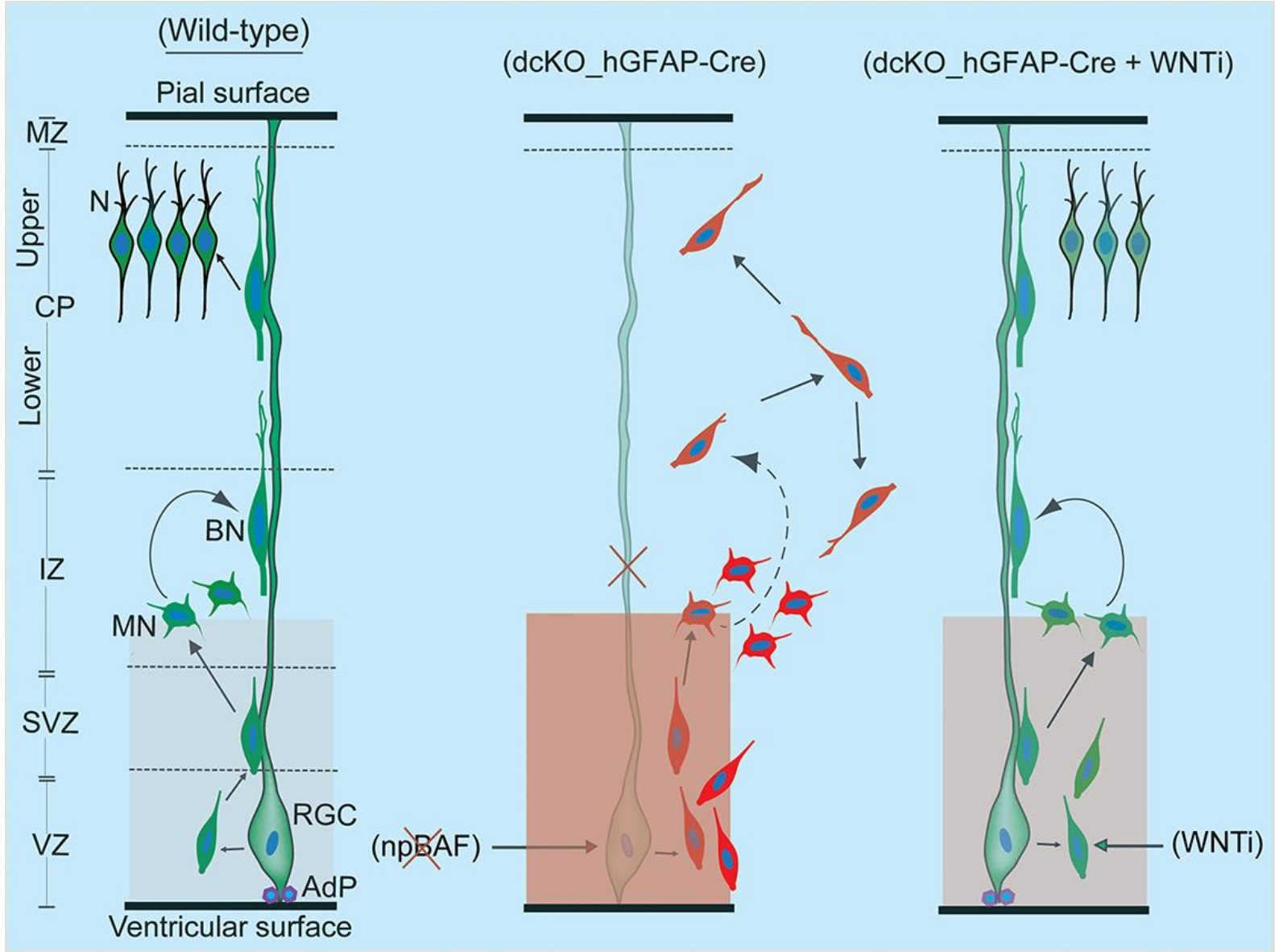

Figure 4.7 Schematic synopsis of how BAF complex mainly influences radial neuronal migration: (a) Schema showing the normal course of radial neuronal migration in the developing wild-type cortex. The parent cortical neural progenitor that generates majority of excitatory neurons is the radial glia cell (RGC). It is typically anchored at the ventricular surface by adhesion proteins (AdP) and extends a long slender fiber that traverses the marginal zone (MZ) to be anchored at the pial surface. The fiber acts as scaffold that supports the radially migrating neuron. After the RGC gives rise to a newborn neuron in the ventricular zone (VZ), the young postmitotic neuron usually attaches to the parent glial fiber and quickly migrates into the border between the subventricular (SVZ) and intermediate zones (IZ), where it acquires a multipolar neuronal (MN) identity and briefly stops migration to receive spatiotemporal molecular cues for further migration. The MN then switches to bipolar neuron (BP) and re-attaches to the glial fiber migrate into the cortical plate $(\mathrm{CP})$. In the $\mathrm{CP}$, the migrating neuron detaches from the glial fiber to under somal translocation leading to its correct laminar placement and to become mature neuron $(\mathrm{N})$. The VZ normal expression levels of WNT signaling is indicate by very light shade of red. (b) Schema showing that loss of BAF complex in neural progenitors (npBAF) causes loss of AdP and glial fiber, disturbance 
of MN-to-BN transition, and increase in WNT signaling activity in the VZ, indicate in deep red. These alterations result in accumulation of young neurons and $\mathrm{MNs}$, leading to abnormal migration and loss of cortical laminar. (c) Picture showing outcome of rescue experiment following inhibition of the increased WNT signaling in the BAF complex mutant (dcKO_hGFAP-Cre) cortex. WNT inhibition with the chemical ICG001 led to substantial preservation of glial fiber and adhesion proteins leading to partial rescue of radial neuronal migration and cortical lamination. Red cross denotes deletion or loss.

\subsection{Discussion}

\subsubsection{BAF complex orchestrates cortical lamination and morphogenesis via regulating neuron migration in the developing cortex}

Laminar patterning during cortical development is fundamental to the formation of functional cortical areas during development of the cerebral cortex. Neuronal migration is pivotal to cortical lamination and formation, and its dysregulation can render the cortex malformed with several resultant neurodevelopmental disorders (Valiente \& Marin, 2010; Evsyukova et al., 2013; Silva et al., 2019). Therefore, factors that regulate migration of cortical neurons have been of interest to many neurobiologists over the years.

In this study, we identified the ATP-dependent chromatin remodeling BAF complex to play instructional roles during radial migration of cortical neurons. We essentially inactivated the BAF complex in neurogenic cortical progenitors or in nascent postmitotic cortical neurons prior to commencement of radial migration. This was carried out using three different mouse cortex models, two of which the BAF complex was inactivated in cortical progenitors at early and later cortical development stages to produce the dcKO_Emx1-Cre and dcKO_hGFAP-Cre, respectively. Abrogation of the BAF complex just after generation of postmitotic cortical neurons was achieved in the dcKO_Nex-Cre model. Thus the dcKO_Nex-Cre model offered the advantage of precluding the impact of abnormal proliferation and neurogenesis seen/reported in the 
dcKO_Emx1-Cre and dcKO_hGFAP-Cre cortical models (Narayanan et al., 2015; Nguyen et al., 2018). In principle, given that the composition and functional characteristics of the BAF complex in neural progenitors (npBAF) is different from that found in neurons (nBAF) (Lessard et al., 2007; Wu et al., 2007; Staahl et al., 2013; Bachmann et al., 2016), to a large extent, we were able to dissect the significance of BAF complexes in orchestrating neuronal migration during cortical histogenesis.

By temporally and cell-type specifically inactivating the BAF complex in the developing cortex, we found that the resultant mutant neurons are incapable of normal migration. As a result, the BAF complex-ablated developing cortex is improperly laminated; showing both upper and lower layer neuron misplacement in the cortical wall. The defective neuronal migration phenotype was severest and morphogenically most impactful in the dcKO_Emx1-Cre cortex followed by the dcKO_hGFAP-Cre cortex. This may be as a result of concurrent disturbance of neurogenesis due to deletion of BAF complex in the early or late cortical neural stem/progenitor cells. Indeed, neural progenitor cell proliferation and differentiation are fettered in the absence of optimal BAF complex function (Narayanan et al., 2015; Nguyen et al., 2018). On the other hand, the migration phenotype in the dcKO_Nex-Cre cortex was mild and mainly presented as delayed locomotion of upper layer neurons because they are late-born and make the longest radial journey. This reduced impact may have resulted from the relative stability of the BAF complex in postmitotic neurons probably because of their non-dividing nature and the non-requirement of subunit recomposition of the nBAF therein (Lessard et al., 2007; Wu et al., 2007). As such, although majority of the BAF subunits are lost, we found some subunit remnants in the P1 dcKO_Nex-Cre cortex (Fig. S4.2). 
Typically, abnormal neuronal migration calls forth several other neurodevelopmental perturbations (Valiente \& Marin, 2010; Evsyukova et al., 2013). In our case, we found that the defective cortical neuronal migration may, in part, underscore the loss of neuronal morphology in the BAF complex mutant cortex which culminated in diminished corticogenesis (Fig. S4.3; Fig. S4.4). At least, in terms of differentiation, some BAF complex subunits have been reported to be essential for axonogenesis, dendritogenesis, and spine formation during cortical neuron maturation (Reviewed in (Sokpor et al., 2017). It is conceivable that in the absence of such differentiationpromoting BAF complex subunits, the mutant developing cortex may attempt to make compensation via alternative schemes leading to increased expression of genes that encourage neuronal maturation such as Robo3 as seen in our RNA-seq results (Fig. 4.3D; (Friocourt \& Chedotal, 2017)). Such developmental rescue effort was however not sufficient to forestall dysgenesis of the cortex as the cortical size is reduced and the corpus callosum is malformed in the BAF complex mutant forebrain.

While it is true that there is a choreography of several ATP-dependent chromatin remodeling factors or complexes at gene enhancers and promoters that sculpt the epigenetic environment, many of them perform unsubstituted roles that conferring some functional specificity during cortical development (Sokpor et al., 2018). For instance, whereas it is not known for the other chromatin remodeling factors, the BAF complex antagonizes the polycomb repressor complex (Ho et al., 2011; Kadoch et al., 2017). As a result, in the absence of BAF complex, the polycomb complex is hyperactive, leading to augmented installation of the repressive mark $\mathrm{H} 3 \mathrm{~K} 27 \mathrm{me} 2 / 3$ on gene bodies with a transcription-silencing effect in the developing forebrain (Narayanan et al., 2015; Nguyen et al., 2016; Nguyen et al., 2018). It is in this direction 
that we found in this study specific migration-related genes to be switched off in the BAF complex-deficient developing cortex. Another reason that consolidates the specific link of BAF complex dysfunction to the observed neuronal migration problem is that, it is only under the condition of entire BAF complex ablation that we disturb cortical neuron migration. Single deletion of BAF155 or BAF170 did not yield any noticeable neuronal migration anomaly in the developing cortex (Tuoc et al., 2013; Narayanan et al., 2018). That notwithstanding, a previous study reported defective neuronal migration following deletion of Ctip1 (BAF100a), a variable subunit of the BAF complex (Wiegreffe et al., 2015). We argue, however, that BAF100a may act solitarily outside the BAF complex to control aspects of cortical neuron migration. Even though the defective migration caused by deletion of BAF155 and BAF170 is more complex and severer than that resulting from BAF100a ablation, it would be insightful to investigate the structural and functional integrity of the BAF100a-lacking BAF complex and how it may contribute to defective neuronal migration.

\subsubsection{Essential cellular mechanics in radial neuronal migration require BAF complex function}

Classically, in utero electroporation is a powerful in vivo technique use for gene manipulation to investigate cellular processes, including neuronal migration in the developing cortex (Saito \& Nakatsuji, 2001; Tabata \& Nakajima, 2001; Shimogori \& Ogawa, 2008). We took advantage of this method to focally delete BAF complex in the developing cortex so as to detail the effect on cellular mechanisms during neuronal migration. To closely reproduce loss of BAF complex in cortical progenitors as achieved in the dcKO_Emx1-Cre and dcKO_hFGAP-Cre cortex, and in postmitotic neurons as in the dcKO_Nex-Cre cortex, we used the CAG- and NeuroD-Cre plasmid 
to ablate BAF155 and BAF170 in selected cortical areas, respectively. By this means, we identified that key steps involved in glial fiber-guided cortical neuron migration go awry in the absence of BAF complex. Notably, we found that the BAF complex-deficient migrating neuron is unable to (properly) attach to and use glial fibers for locomotion. The observed reduction in neuronal-glial fiber interaction may partly be due to disturbance in cell adhesion to available glial fibers as revealed in our RNA-seq analyses of the dcKO_Emx1-Cre and dcKO_hFGAP-Cre cortex. Adhesion molecules such as catenins and cadherins, play central roles in the attachment of neurons to glial guides during locomotion (Solecki, 2012). Therefore, it has been recently shown that loss of adhesion proteins in the cortex, particularly $a-C a t e n i n$, can perturb radial neuronal migration leading to cortical neurodevelopmental disturbances (Schmid et al., 2014; Schaffer et al., 2018). This partly gives relevance to the link between loss of $\alpha$ Catenin in the BAF complex-inactivated cortex and contribution to the defective radial migration, and cortical mis-lamination phenotype reported in this study.

Although not obviously revealed in our BAF complex knockdown investigation using in utero electroporation, it is conceivable that the reduced association of the BAF complex-lacking migrating cortical neurons with glial fibers may also emanate from the absolute loss of the glial fiber layouts needed for radial migration. At least, this is true for the dcKO_Emx1-Cre and dcKO_hFGAP-Cre cortex which display dramatic loss of glial fiber scaffolds due to ablation of BAF complex (Fig. 4.1D/E, Fig. 4.2C/D). Traditionally, nascent cortical neurons use their parent glial fibers to locomote to the CP to make well-patterned radial columns, cortical laminae, and functional areas defined in the cortex (Mountcastle, 1997; Noctor et al., 2001; Torii et al., 2009). Neuronal migration may stall or deviate in the absence of such proximal glial fiber guide which led to the accumulation of Ctip2+ and Cux1+ neurons in the lower cortical wall 
of the dcKO_hGFAP-Cre cortex and perhaps in the germinal zones of the cortical area electroporated with CAG-Cre-GFP plasmid (Fig. 4.4C/D). Moreover, our observation indicates that the migrating BAF-ablated neurons may display protracted multipolar phase leading to their accumulation in the $\mathrm{IZ}$ and/or may undergo excessive tangential migration to find adjoining fibers for onward radial migration (Inoue et al., 2017). This can engender unnecessary lateral dispersion of the migrating neurons as apparent in our BAF complex knocked-down cortex (Fig. 4.4B).

Adding to the complexity of how BAF complex regulates cortical neuron migration, we also identified its importance in controlling the multipolar-to-bipolar neuronal morphology switch during radial migration. This morphological transformation is critical for successful radial migration (Noctor et al., 2004; Ayala et al., 2007; Namba et al., 2014) and its interference or dysregulation can stifle neuronal migration (La Fata et al., 2014; Boitard et al., 2015; Chen et al., 2015; Barnat et al., 2017; Guo et al., 2017; Huang et al., 2017; Iwai et al., 2018; Kurabayashi et al., 2018; Ohtaka-Maruyama et al., 2018). The BAF complex-ablated cortical neurons may not be able to properly undergo multipolar-to-bipolar transition leading to accumulation of neurons at the multipolar stage and fewer successfully migrated neurons in the CP. We posit that neuronal polarization is fundamentally distorted in the absence of BAF complex. In support of this notion, we found downregulation of the cytoskeleton and related factors in the BAF complex-deleted cortex. More so, adhesion proteins like a-catenin which orchestrate the plastic linkage between the cell membrane and the internal cytoskeleton to afford cell (neuronal) structure remodeling (Drees et al., 2005; Desai et al., 2013), is lost in response to BAF complex inactivation in the developing cortex. Our investigation of Golgi apparatus localization, which can also indicate alteration in cell polarization, corroborates aberrant neuronal polarization and resultant disturbance 
of multipolar-to-bipolar morphology conversion consequent to BAF complex deletion. A common consequence of improper neuronal polarization during migration is the disturbance of axonal and dendritic extension, which in our study were observed as truncation of leading process (future dendrite) length and drastic reduction in intercortical axonal connection in the BAF complex mutant cerebral cortex.

Together, our investigations show that the BAF complex modulates contact guidance necessary for radial neuronal migration and cortical laminar formation. Key among them is that the BAF complex is essential for maintenance of RG fiber scaffolds, cell adhesion, and neuronal polarization that are indispensable for locomotion of cortical neurons and overall cortical ontology.

\subsubsection{BAF complex may suppress WNT signaling to drive cortical neuron migration}

Despite the mechanistic cellular intricacies involved in the regulation of cortical neuron migrations by the ATP-dependent chromatin remodeling BAF complex, we were determined to identify a unified molecular mechanism through which BAF complex acts to control radial neuronal migration and resultant cortical laminar formation in the developing brain. The challenge begun by screening for possible molecular candidates imputable to our BAF complex-induced neuronal migration phenotype. The WNT signaling pathway emerged as the most plausible candidate among a cacophony of factors altered in the BAF complex mutant cortex. Unlike other identified signaling pathways altered in the BAF mutant cortex, WNT signaling appeared elevated in all three dcKO models applied in the study. Moreover, the disturbance of WNT signaling and its effectors are known to cause abnormal neuronal migration (Poschl et al., 2013; Boitard et al., 2015; Bocchi et al., 2017). Admittedly, while WNT activity is markedly 
increased in the dcKO_Emx1-Cre (see RNA-seq data sheet in (Narayanan et al., 2015)) and dcKO_hFGAP-Cre cortex (this study; (Nguyen et al., 2018)), the dcKO_Nex-Cre cortex displayed mild elevation in WNT signaling probably due to alteration in indirect WNT signaling effectors, including Rspo2 (Fig. 4.3D; (Lebensohn \& Rohatgi, 2018)). Perhaps, the perturbation in WNT signaling in the dcKO_Nex-Cre cortex was mild enough to have allowed some degree of cortical layer formation at later postnatal stages of cortical development, which was not the case in the dcKO_Emx1-Cre or dcKO_hFGAP-Cre cortex.

By downregulating the increased WNT activity in the BAF mutant (dcKO_hFGAP-Cre) cortex, we were able to substantially obviate abnormal radial migration of neurons. The rescue also included re-emergence of glial fibers and at least the adhesion molecule, $\alpha$-catenin. Thus, our rescue experiment showed that the BAF complex likely modulates WNT signaling to allow optimal establishment of the requisite molecular and cellular conditions for normal oriented neuronal migration and proper patterning of neocortical layers. The possible explanation for the WNT inhibition-mediated rescue of migration reported here is that BAF complex may repress WNT signaling in neurons en route to their laminae in the CP. In support of our hypothesis, it was previously shown that BAF complex can inhibit WNT/ß-catenin signaling via its subunit BAF250b (Vasileiou et al., 2015). It was also reported that WNT signaling is dynamically regulated to allow correct neuronal polarity formation and neuron-glial fiber engagement during radial migration (Boitard et al., 2015; Bocchi et al., 2017). When WNT signaling was ablated in the developing cortex, it resulted in delay of radial migration leading to reduction in interhemispheric connection and cortical size (Boitard et al., 2015; Bocchi et al., 2017)), a phenotype recapitulated in our dcKO_hGFAP-Cre and dcKO_Nex-Cre cortices. WNT signaling is indeed a formidable regulator of cortical development as it is reported to 
regulate many aspects of brain development, including primary forebrain patterning, $R G$ and neuronal precursor cell fate, cell adhesion and polarity formation, and cortical laminar patterning (Reviewed in (Harrison-Uy \& Pleasure, 2012)).

Altogether, this current study highlights the indispensability of the ATP-dependent chromatin remodeling BAF complex in the formation of cortical layers through regulating multiple aspects of radial neuronal migration in a WNT signaling dependent manner during mammalian cortical development.

\subsection{Material and Methods}

\subsubsection{Animal handling and generation of transgenic mice}

We applied guidelines of the German Animal Protection Law in handling the animals. Floxed BAF155 (Choi et al., 2012), floxed BAF170 (Tuoc et al., 2013), Emx1-Cre (Gorski et al., 2002), hGFAP-Cre (Zhuo et al., 2001), and Nex-Cre (Goebbels et al., 2006) were the transgenic mice used in the study. All animals were maintained in a C57BL6/J background.

To eliminate BAF155 and BAF170 in early or late cortical progenitors, or projection neurons, we crossed mice carrying floxed BAF155 and BAF70 genes with the early progenitor-active Emx1-Cre (Gorski et al., 2002), late progenitor-active hGFAP-Cre (Zhuo et al., 2001) and neuron-specific Nex-Cre (Goebbels et al., 2006) mouse lines to generate dcKO_Emx1-Cre, dcKO_hGFAP-Cre, and dcKO_Nex-Cre cortical mutants, respectively. Heterozygous animals (BAF155fl/+, BAF170fl/+, Cre negative) were used as controls. Emx1-Cre mutants die before birth, hGFAP-Cre and Nex-Cre mutants survive early postnatal stages. 


\subsubsection{Plasmids}

The following plasmids were used in the study: pCIG2-ires-eGFP, pCIG2-Cre-ireseGFP (gift from Prof. Francois Guillemot, NIMR London; (Hand et al., 2005)), and NeuroD-Cre-ires-GFP, NeuroD-GFP (gift from Prof. Laurent Nguyen, University of Liège, CHU Sart Tilman, Liège, Belgium).

\subsubsection{Antibodies}

Commercially obtained monoclonal (mAb) and polyclonal (pAb) primary antibodies used in the study: CTIP2 rat pAb (1:200; Cat. ab18465; Abcam), Cux1 rabbit pAb (1:50; Cat. sc-13024, Santa Cruz), Brn2 goat pAb (1:100; Cat. sc-6029, Santa Cruz), GM130 rat mAb (1:100; Cat. 610823; BD), L1 rat mAb (1:500; Cat. MAB5272; Chemicon), BAF170 rabbit pAb (1:100; Cat. HPA021213; Sigma), BAF60a mouse mAb (1:200; Cat. 611728; BD), BAF155 rabbit pAb (1:20; Cat. sc-10756; Santa Cruz), BRM rabbit pAb (1:200; Cat. ab15597; Abcam), BRG1 rabbit pAb (1:120; Cat. sc-10768X; Santa Cruz), BAF155 mouse mAb (1:100; Cat. sc-48350X; Santa Cruz), BAF250b mouse mAb (1:100; Cat. WH0057492M1; Sigma), Tbr1 rabbit pAb (1:300; Cat. ab31940; Abcam), HuCD mouse mAb (1:20; Cat. A21271; Invitrogen), $\alpha$-Catenin rabbit pAb (1:200; Cat. C2081; Sigma), PAX6 mouse mAb (1:100; Developmental Studies Hybridoma Bank), Nestin mouse mAb (1:50; Cat. 611658, BD), PAX6 rabbit pAb (1:200; Cat. PRB-278P; Covance), GFP chicken pAb (1:500; Cat. ab13970; Abcam). Secondary antibodies used were Alexa 488-, Alexa 568-, Alexa 594- and Alexa 647conjugated IgG (various species, 1:400; Molecular Probes). 


\subsubsection{RNA sequencing}

RNA sequencing and analyses were performed as previously described in (Narayanan et al., 2015; Narayanan et al., 2018; Nguyen et al., 2018).

\subsubsection{Cortical size estimation}

We adopted some previously described cortical size measurement and analyses approaches (Bishop et al., 2003; Bishop et al., 2002; Sahara and O'Leary, 2009; Tuoc et al., 2013). Briefly, the dorsal view of mutant and control brains were photomicrographed in bright field with a Stereo microscope (Leica M205 FA). Quantitative comparison of estimated control and mutant cortical size was done by measuring the outlined cortical surface and demarcated anterioposterior axis $(A / P)$ of the imaged brains using NIH ImageJ software.

\subsubsection{Immunohistology and in situ hybridization}

Immunostaining and in situ hybridization of cortical tissue were performed as previously described (Tuoc et al., 2013; Bachmann et al., 2016).

\subsubsection{Imaging and quantitative analysis}

Anatomically comparable stained coronal brain sections were imaged with confocal (TCS SP5, Leica) and/or fluorescence (Axio Imager M2, Zeiss; fitted with Neurolucida software, MBF Bioscience) microscopes. Further image processing was done with Adobe Photoshop program.

Neuronal cell counting and distribution (bin analysis), and leading process length estimation were performed using NIH ImageJ software. Neuronal cells with nuclear or 
cytoplasmic labeling for specific markers with or without DAPI background staining were counted in 4-6 structurally-matched control and mutant (dcKO) or electroporated cortical sections obtained from 3-4 biological replicates. Fluorescent signal intensity measurement in the form of mean pixel value estimation was used to quantified uncountable histological staining using $\mathrm{NIH} \mathrm{ImageJ} \mathrm{software,} \mathrm{as} \mathrm{described} \mathrm{previously}$ (Narayanan et al., 2015; Tuoc and Stoykova, 2008).

\subsubsection{In utero electroporation}

In utero electroporation was done as previously described (Tabata \& Nakajima, 2001; Tuoc \& Stoykova, 2008; Tuoc et al., 2013).

\subsubsection{WNT inhibitor pharmacological treatment}

ICG001 (Tocris Bioscience, Cat. No. 4505), was dissolved in DMSO (vehicle). Pregnant mice received daily intraperitoneal injections of vehicle $(150 \mu \mathrm{l})$, or ICG001 (150 $\mu \mathrm{l}$ of a $1.0 \mathrm{mg} / \mathrm{ml}$ solution) from $11.5-16.5$ days post coitum (d.p.c.). The brains of the treated embryos (control and mutant) were harvested and processed for histological analysis.

\subsubsection{Statistical analyses}

Statistical comparisons were carried out using Student's $t$ test and its non-parametric version (Mann Whitney Test), and one-way ANOVA followed by Bonferroni's multiple comparison test where appropriate. The results are presented as means \pm SEM. All details of statistical analyses for histological experiments are presented in Table S4. 


\subsection{Acknowledgments}

We acknowledge Fett H., and Huttanus T. for their expert animal care and support. We also thank Nguyen L., and Guillemot F. for sharing reagents and plasmids with us. This work was supported by the Universitätsmedizin Göttingen (T.T), TU432/1-1, TU432/13 DFG grants (T.T), Schram-Stiftung (T.T), DFG-CNMPB (T.T, J.F.S), DAAD-MoE Ghana Scholarship (G.S), and partly by the Goettingen Graduate School for Neuroscience, Biophysics and Molecular Bioscience (DFG Grant GSC 226/4) (G.S).

\subsection{Author contributions}

G.S. performed most phenotype characterization, and all statistical analyses; C.K. generated RNA sequencing data. L.P., H.N., and J.R. contributed to histological analyses; R.W. did FISH experiment and provided data in figure 4.3A; J.F.S. provided research tools, transgenic lines, and contributed to discussions; T.T. conceived, and supervised the study; G.S. and T.T. wrote the manuscript. The authors declare no competing financial interests. 


\subsection{Supplemental figures}

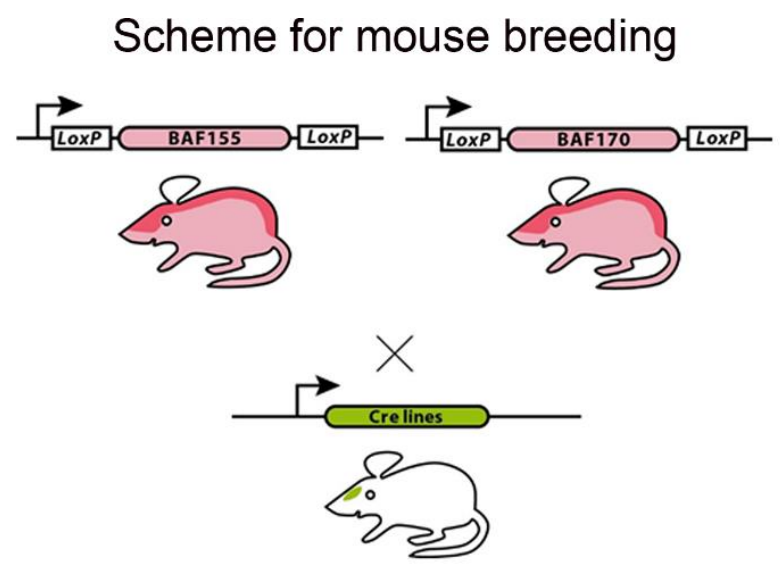

Emx1-Cre

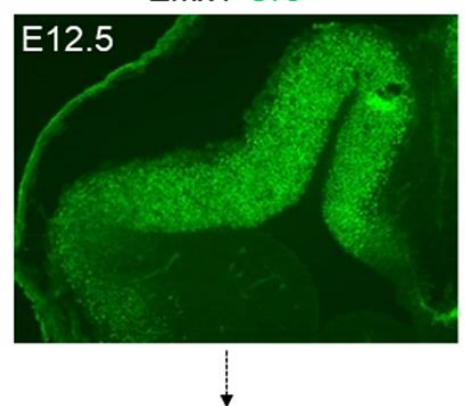

Early cortical progenitorspecific dcKO_Emx1

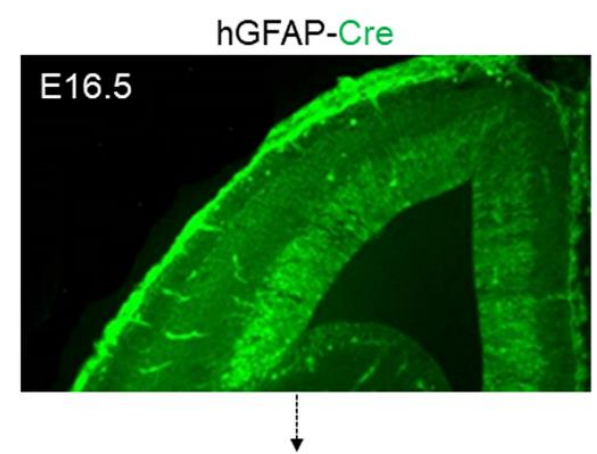

Late cortical progenitorspecific dcKO_hGFAP

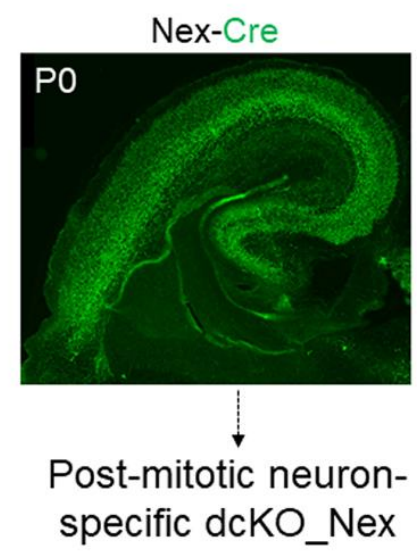

Figure S4.1: Picture showing schema depicting the generation of the double conditional knockout (dcKO) mice used in the study and the corresponding immunohistochemical micrographs indicating Cre recombinase activity at the specified cortical development stages. 

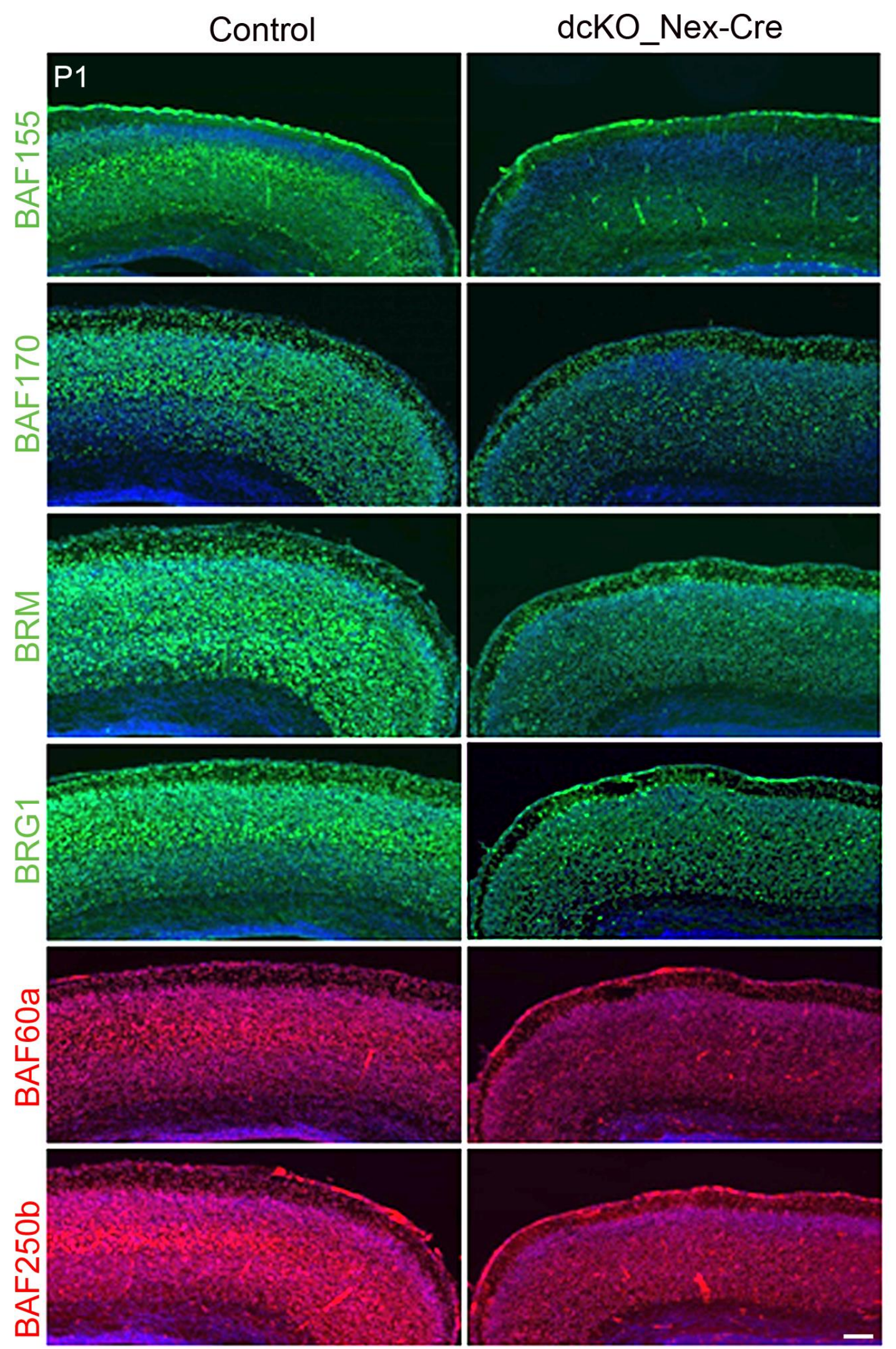

Figure S4.2: Micrographs showing immunostaining of the various indicated BAF complex subunits in the P1 control cortex and relative depleted levels in the dcKO_Nex-Cre cortex. Scale bar $=100 \mu \mathrm{m}$. 
A

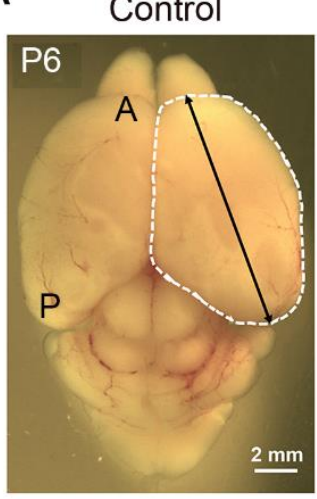

B

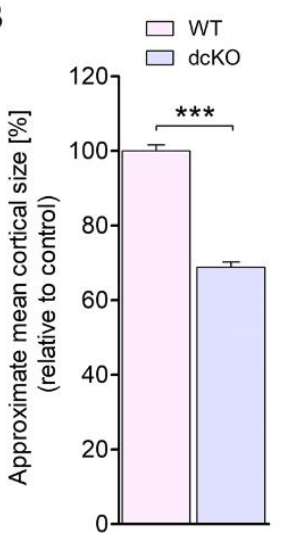

dcKO_Nex-Cre

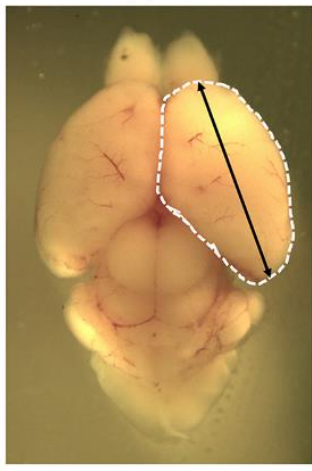

C $\square$ WT

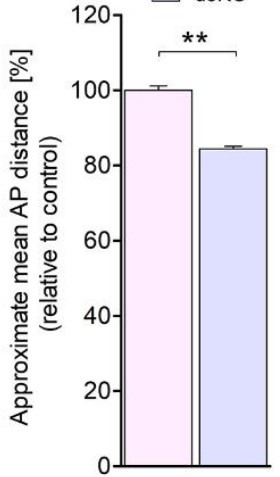

D

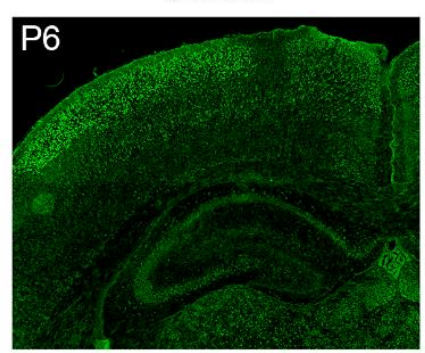

Control
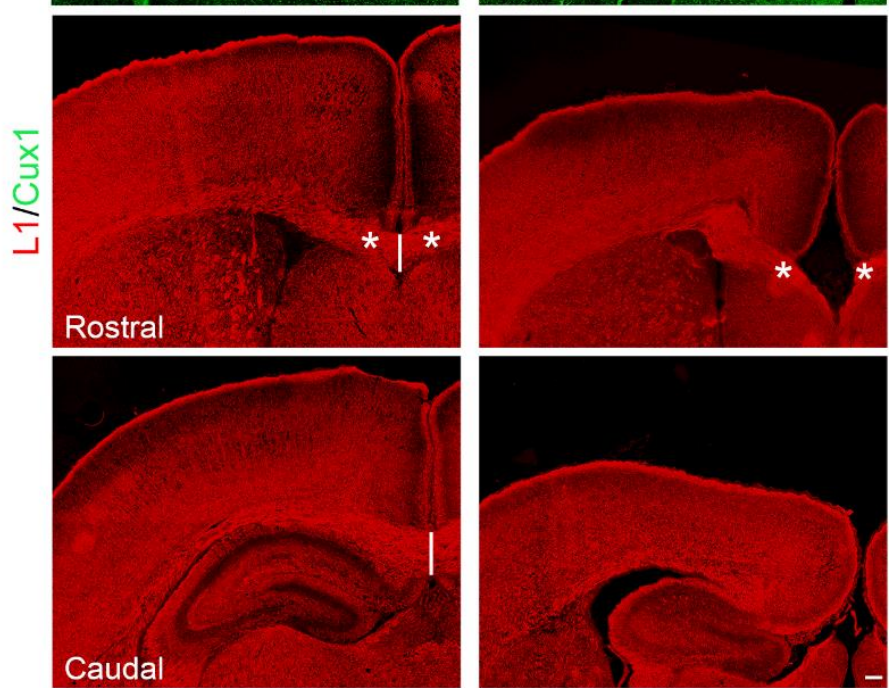

dcKO_Nex-Cre
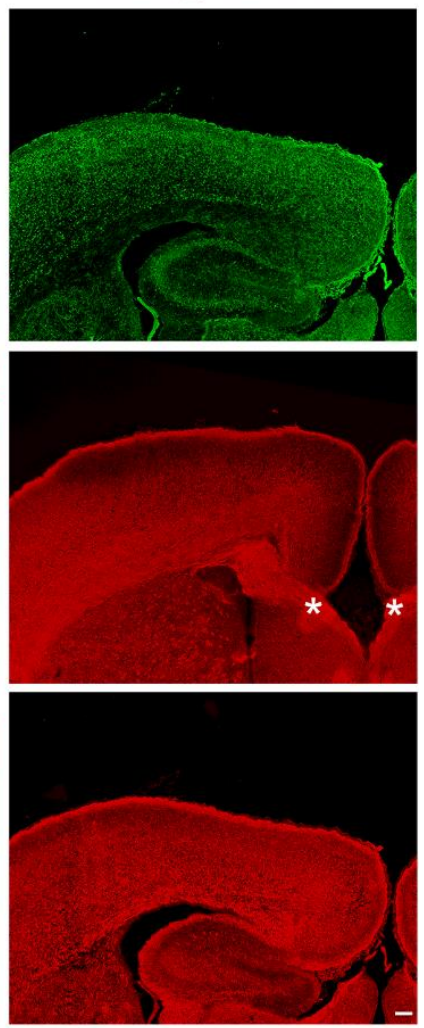

Figure S4.3 Loss of neuronal BAF complex causes cortical hypoplasia and corpus callosum defects: (A) Image showing overview of the P6 control and dcKO_Nex-Cre brain in bright field. The approximate area and anterioposterior dimension of one cortical hemisphere are demarcated with white dashed lines and black double-end arrows, respectively. (B, C) Bar charts showing quantitative comparison between the estimated cortical size (B) and anterioposterior distance (C) of the control and mutant brain. (D) Micrographs showing immunostaining of upper layer neurons and the corpus callosum in coronal sections of the P6 control and dcKO_Nex-Cre cortex with Cux1 and L1 antibodies, respectively. The right and left aspects of the corpus callosum are asterisked, and the midline corpus callosum thickness is indicated by white vertical line. Scale bars: $2 \mathrm{~mm}$ (in A) and $100 \mu \mathrm{m}$ (in D). Abbreviations: Anterior pole (A), Posterior pole (P). 


\section{CHAPTER 5: General discussion}

\subsection{Maintenance of BAF complex integrity is essential for optimal brain morphogenesis}

The BAF complex has been shown to be a phenomenal regulator of many cell biological processes during neurodevelopment. As a result, the mammalian forebrain receives a fair share of the mechanistic involvement of BAF complex during development (Sokpor et al., 2017). By means of partial or complete ablation of the BAF complex, we have been able to identify alterations in the epigenetic landscape which have consolidated our understanding of key mechanistic axes along which the BAF complex operates. Particularly, in the case of complete abolishment of the BAF complex function achieved via double deletion of the core BAF subunits 155 and 170 during cortical development, our work in chapter one (Nguyen et al., 2016) and previous investigation (Narayanan et al., 2015) indicate changes in chromatin state which mainly does not support optimum gene expression programs needed for cortical development.

Specifically, we unraveled that BAF155 and BAF170 are critical for the maintenance of the stability of the entire complex. Therefore, in their absence, the other BAF complex subunits disassemble or are aberrantly assembled leading to their targeting by the ubiquitin-proteasome machinery for degradation. As a result, there is a global elevation in the level of the heterochromatin histone methyl marks H3K27me2/3 in the developing BAF complex-deficient forebrain (Jung et al., 2012; Narayanan et al., 2015; Nguyen et al., 2016). The BAF complex normally works in opposition to the H3K27 methylation complex (PRC2) to cooperatively modulate chromatin methylation schemes and maintain associated homeostasis (Ho et al., 2011; Kadoch et al., 2017). 
In other words, whereas the BAF complex function frequently results in euchromatin (active chromatin) state with the help of the demethylase enzymes UTX (KDM6A) and JMJD3 (KDM6B), the H3K27 methyltransferase Ezh2 (a PRC2 subunit), promotes heterochromatin (inactive chromatin) state (Narayanan et al., 2015; Nguyen et al., 2016). Hence, in the absence of BAF complex, the balance is tipped in favor of chromatin condensation which is generally associate with downregulation of gene expression and resultant developmental anomalies (Fig. 2.4; (Nguyen et al., 2016)).

Interestingly, it became evident and more concrete from our recent study (Nguyen et al., 2018) that the effect of the distortions in the epigenetic milieu following deletion of BAF155 and BAF170 is bimodal in terms of gene expression as previously suggested (Narayanan et al., 2015). We found that loss of BAF complex causes the repression of a set of corticogenic genes while promoting the expression of other categories of genes required for late cortical development (Nguyen et al., 2018). Thus, not only transcription-repressing epigenetic histone methyl marks such as H3K27me3 predominate in the absence of BAF complex, but also transcription-activating marks like H3K4me2. In effect, increased proliferation of neural progenitors and decreased neurogenic differentiation coexist in the BAF complex-inactive developing cortex leading to perturbations in cortical formation (Nguyen et al., 2018).

The prominent expression of BAF complex in the mammalian central nervous system during development is indicative of its significance in regulating neural development, including ontology of the brain (Nguyen et al., 2016). This is partly supported by the existence of many syndromic and non-syndromic neurodevelopmental disorders traceable to malfunctioning of the BAF complex or its constituent subunit(s) during brain development (Sokpor et al., 2017). Our contribution towards elucidating the role 
of BAF complex in forebrain development is basically captured in the idea that the stability, and therefore proper function of the BAF complex, is largely dependent on the co-functionality of it subunits BAF155 and BAF170. Thus, interference of this dual subunit functionality compromises the capacity of the BAF complex to orchestrate discrete neurodevelopmental processes involved in brain development.

Nonetheless, it is worth mentioning that there are other ATP-dependent chromatin remodeling complexes other than the BAF complex that play prominent roles in cortical neurogenesis (Sokpor et al., 2018). Although none of these other chromatin regulators has yet been identified to control cortical patterning, it is not far-fetched to consider the possibility, given that some seem to slightly overlap in functionality compared to the BAF complex during cortical histogenesis (Sokpor et al., 2018). Further study is recommended to determine if and/or how the other chromatin remodelers contribute to cortical patterning, and how they operate in concert with the BAF complex to achieve a holistic epigenetic regulation of cortical formation during brain development.

\subsection{Featuring the chromatin remodeling BAF complex in the regulation of cortical patterning}

Proper patterning of the mammalian forebrain is important for the correct establishment of the various structural components and functional areas of the cortex. Regionalization of the dorsal aspect of the telencephalon results in the major demarcations in the cortex namely the medial, dorsal, and lateral aspects-where the medial cortex gives rise to the hippocampus and other archicortical structures and the dorsolateral cortex essentially become the neocortex. Cortical arealization on the other hand leads to the functional areal parcellation of the cortex to produce the motor cortex and the various sensory cortical areas (S1, A1, and V1). The inception of early cortical 
regionalization is mainly triggered by morphogens released from the cortical organizers, including the cortical hem, whereas the areal maps of the cortex are basically formed under the control of transcription factors with gradient expression (O'Leary et al., 2007). Disturbance in secretion of the morphogen or mis-expression of the transcriptional factors involved in cortical patterning is linked to abnormal patterning of the cortex and related cortical dysfunction. However, how hierarchical regulatory events such as chromatin remodeling contribute to the orchestration of cortical patterning schemes are unknown.

The study in chapter 3 unraveled an originative role for BAF complex in early patterning of the dorsal telencephalon. Briefly, we posit that the BAF complex is a modulator of dorsal telencephalic patterning through suppressing unwarranted expansion of the cortical hem. We have for the first time shown how specific chromatin remodeling activity translates into the proper subdivision of the dorsal telencephalon through the transactivation of key transcriptional programs know to instruct regional and areal patterning of the developing cortex. Cortical region patterning goes awry when the regulatory function of the BAF complex is excluded from the pool of mechanisms known to control forebrain patterning. The cortex is grossly misshapened when the BAF complex is deleted from the cortical primordium. Regionally, the BAF complexdeficient cortex is not properly subdivided. The medial cortex predominates the cortical identity in the absence of BAF complex function. The abnormally patterned medial cortex however lack the hippocampal primordium. We attribute the cortical mispatterning to abnormal expansion of the cortical hem which is the organizer of medial cortical fate and maintains neocortical identity (Caronia-Brown et al., 2014). It means that, the expanded cortical hem loses its capacity to instruct progenitors in the adjoining cortical neuroepithelium to adopt the appropriate medial cortical fate. It is also likely 
that the BAF complex mutant hem is not able to properly generate its cellular derivative(s). It can therefore be inferred that the BAF complex maintains the functional integrity of the cortical hem via limiting its abnormal expansion.

Mechanistically, we found that the BAF complex cooperates with the transcription factor LHX2 to cause proper delineation of the medial cortex from the neocortex. That is to say that the BAF complex may be a determinant of medial cortical identity, an idea partly supported by a recent study (Filatova et al., 2019). The exact mechanistic relationship between the BAF complex and LHX2 during regional patterning is however unclear, although our investigation so far indicates that BAF complex may potentiate the transcriptional activity of LHX2 but not necessarily at the gene level since Lhx2 is still expressed in the absence of BAF complex. Although, we did not find a direct interaction between BAF complex and Foxg1, an upstream regulator of Lhx2 during cortical patterning (Godbole et al., 2018), it is possible that a modulatory triad relationship may exist among them during regional patterning of the cortex.

It is conceivable that the BAF complex is also involved in cortical arealization. This conception is founded on our observation that Pax6 expression and/or transactivation is under the control of BAF complex. As mentioned in chapter one, Pax6 dosage during cortical areal pattering is vital for the correct arealization of the cortex. Complete loss of Pax6 in the cortex causes abnormal expansion of the $\mathrm{V} 1$ cortical area at the expense of the size and location of other cortical areas. Even a partial reduction in the amount of Pax6 in the developing cortex of Yac-Pax6 transgenic mice (i.e., Pax6 heterozygosity) resulted in slight enlargement of $\mathrm{S} 1$, albeit the other cortical areas remained unchanged (Zembrzycki et al., 2007). Meanwhile, Pax6 is also a gene target of LHX2 transcriptional activity (Hou et al., 2013). Therefore, it is plausible that BAF 
complex directly or indirectly (via Lhx2 regulation) participates in the areal parcellation of the cortex into the various functional domains.

Evolutionarily, it is expected that some factors that orchestrate developmental processes that confer ontological advancement in higher mammalian classes gain some form of functional refinement or upgrade to be able to execute "higher" developmental events. Although our investigation of the role of BAF complex in mediolateral cortical patterning was established in the rodent cortex, we found a plausible refined role for BAF complex in performing more sophisticated cortical patterning processes in the primate cortex. Unlike in the mouse dorsal telencephalon, the BAF complex subunits are expressed in a graded concentration in the primate-like Marmoset cortex, a characteristic which typifies the majority of morphogens and transcription factors reported to play important roles in brain patterning. We believe this observation is a core evodevo adaptive change in BAF complex function so that it is able to direct more robust cortical regionalization and arealization in the primate cortex. Further investigation into the exact role of BAF complex in regulating patterning of the primate cortex may reveal key mechanisms the can serve as crucial clues in the design of interventions aimed at mitigating some neurodevelopmental and neuropsychiatric abnormalities that afflict the brain due to BAF complex malfunction (Sokpor et al., 2017).

\subsection{Proper BAF complex-instructed neuronal migration is a blueprint for cortical lamination and formation}

The simple act of generating neurons in the cortical germinal zones is no guarantee for normal progression of corticogenesis. The neurons must move out of their site of production to settle in designated functional loci in the $\mathrm{CP}$ or other cortical areas. The 
active movement of neurons leading to their correct positioning constitute the process of neuronal migration. As extensively covered in the introductory part of this write-up, excitatory cortical neurons mainly migrated radially to reside in the $\mathrm{CP}$ and they form the majority of neurons in the cortex. The remaining neurons, which were not our focus in the study presented in chapter 4 , are mainly inhibitory neurons that migration tangentially from regions outside the cortex, say from the ganglionic eminences in the sub-pallium. Together, these major modes of neuronal migration are indispensable for neuronal organization in the cerebral cortex to permit optimal functionality.

In the context of radial neuronal migration, we have shown that the BAF complex is required for, at least, glia fiber-dependent radial migration of excitatory neurons during active stages of neurogenesis and cortical expansion. BAF complex deprivation in the developing cortex had deleterious effect on cortical layer formation and morphogenesis of the entire mouse forebrain. The preliminary lead to identifying that the BAF complex may be a regulator of neuronal migration originated from our observation that the improperly patterned 13.5 Emx1-Cre BAF mutant dorsal telencephalon (presented in chapter 3) lacked critical factors needed for radial migration, including Cajal-Retzius cells which produce the glycoprotein Reelin needed for cortical lamination (Rice \& Curran, 2001; Takiguchi-Hayashi et al., 2004; Bielle, F. et al., 2005; Liu et al., 2018). Subsequent investigations at more advanced cortical development stages, and under the conditions of BAF complex deletion in progenitors and exclusive in postmitotic neurons, lend clarity to how the BAF complex regulates radial migration of cortical neurons to afford proper layer formation in the developing cortex.

The glia fiber grids with which neurons migrate radially to the CP are lost in the cortex lacking BAF complex. The reason for the fiber lost is likely as a result of abnormal cell 
dynamics of RGs, leading to three possible mode of fiber loss: (i) instability and eventual degeneration of already formed fibers, (ii) inability of RG cells to elaborate their fibers, and/or (iii) abnormal delamination of RG cells from the adherens junction belt at the ventricular surface due to depletion of adherens junction factors, including a-Catenin (Xie et al., 2019). Interestingly, adherens junction molecules themselves are important regulators of neuronal migration. Because they aid adhesion of locomoting neurons to glia fibers, and their depletion frequently disturbs radial migration of neurons (Solecki, 2012).

It is interesting that we found observable disturbance in the formation of the corpus callosum when the BAF complex was exclusively deleted in postmitotic neurons. We think that since such BAF complex mutant neurons, especially upper layer neurons whose axons mainly form the corpus callosum, delay in their migration, their axonal extension or formation (axonogenesis) is hampered. Thus, we strongly link the corpus callosum agenesis seen in the BAF complex mutant mouse or human brain (our current study; (Filatova et al., 2019)) to dysregulation of radial migration of affected neurons because it is during proper radial migration that the trailing process of the migrating neuron gets extended to form the future axon (Nadarajah et al., 2001; Noctor et al., 2004).

At the molecular level, we think that the increased WNT signaling is a major triggering factor of abnormal radial migration in the absence of BAF complex. Although the positive rescue of abnormal radial migration and cortical lamination by WNT signaling downregulation was achieved in the dcKO_hGFAP-Cre cortex, it is plausible that the same experimental rescue scheme applies to the other BAF complex mutant cortices since they also display increased WNT activity. That notwithstanding, restoration of 
abnormal changes in the epigenome of the BAF mutant cortex can possibly reverse the radial migration perturbation and associated distortion in cortical layer formation therein. A specific promising approach in that regard may be to use alternative means of say keeping installation of heterochromatin marks at bay, which is achievable via overexpression of the demethylases UTX and JMJD3. The caveat, however, is that the regulation of radial migration is not due to the non-chromatin functions such as the recruitment of other cofactors implicated in the regulation of radial migration.

Technically, neuronal migration can be considered as a late cortical patterning event which may already be programmed in neural progenitors and required for the so-called laminar patterning of the cortex. Indeed, several brain patterning factors, including WTNs, BMPs, FGFS and Gli3 (a component of the Shh cascade), have been reported to regulate migration of cortical neurons and hence laminar patterning development (Hashimoto-Torii et al., 2008; Boitard et al., 2015; Bocchi et al., 2017; Martinez-Chavez et al., 2018; Saxena et al., 2018). It implies that, by regulating neuronal migration, the BAF complex may simply be doing the work of what other cortical patterning factors do during brain development. Therefore, the BAF complex role in controlling radial migration may not necessarily be an isolated function but an addition to its multiplex functionality in regulating cortical patterning and development. Thus, our discovery of the BAF complex as a regulator of cortical laminar or structure formation via modulation of radial neuronal migration has extended the frontier of the role of chromatin remodelers in cortical development (Sokpor et al., 2017; Sokpor et al., 2018). 


\section{General Summary}

The brain is one of the complex organs designed under the control of a plethora of molecular and cellular regulatory mechanisms, including transcriptional and epigenetic pathways. Brain organogenesis proceeds in a stepwise manner involving both embryonic and postnatal cell biological processes. Such crucial processes include patterning, neurogenesis, and neuronal migration. Patterning of the brain ensures early regional subdivision of the telencephalon and functional areal sculpting of the cortex, neurogenesis is the process of neuronal production, and newly generated neurons migrate to their structural and functional maturation site in the cortex via the process of migration. The ATP-dependent chromatin remodeling BAF complex is one of the powerful epigenetic regulators of several aspects of brain development, although some of its functions thereof are only implicated and not clearly defined. How the BAF complex regulates patterning of the dorsal aspect of the telencephalon and radial glia fiber-dependent neuron migration are the main themes of this dissertation aimed at expanding the neurodevelopmental importance of the chromatin remodelers during cortical histogenesis.

By adopting a conditional genetic manipulation scheme of deleting the two BAF complex scaffolding subunits BAF155 and BAF170, we were able to generate mouse mutants that lacked the expression of BAFs in the cortex. It was observed that early patterning of the dorsal telencephalon that leads to delineation of the midline telencephalic structures such as the hem and hippocampus from the dorsolateral telencephalon (neocortex) is perturbed in the absence of BAF complex. The BAF complex-deficient dorsal telencephalon displayed an expanded hem, no hippocampal primordium, and marked medialization of the ensuing cortex. The BAF complex was identified to cooperate with the transcription and patterning factor LHX2 to drive the 
regional patterning of the dorsal telencephalon. Strikingly, neurons are utterly misplaced in the mis-patterned BAF complex-ablated dorsal telencephalon, raising the possibility of cortical laminar mis-patterning at later stages of forebrain development. Indeed, the late embryonic and early postnatal developing BAF complex mutant cortices are improperly laminated. Abnormal radial neuronal migration was identified to underscore the disturbed layer formation which specifically stemmed from the loss of radial glia fibers/scaffolds, depletion of cell adhesion, and sub-optimal neuronal polarization following deletion of BAF complex in neural progenitors and/or postmitotic neurons. Interestingly, the WNT signaling was shown to be modulated by the BAF complex to afford normal radial migration of cortical neurons. Altogether, these current investigations have provided insights into how the chromatin remodeling BAF complex contributes to cortical morphogenesis through regulating the regional subdivision of the dorsal telencephalon and neuronal placement during the cytoarchitectural organization of the cortex. Our current findings further clarify the multifaceted means by which the BAF complex regulates brain development and offers additional potential targets for therapeutic consideration in neurodevelopmental disorders imputable to malfunction of the BAF complex in the mammalian brain.

\section{References}

Aaku-Saraste E, Hellwig A, Huttner WB. 1996. Loss of occludin and functional tight junctions, but not ZO-1, during neural tube closure-remodeling of the neuroepithelium prior to neurogenesis. Dev Biol 180(2): 664-679.

Aeckerle N, Drummer C, Debowski K, Viebahn C, Behr R. 2015. Primordial germ cell development in the marmoset monkey as revealed by pluripotency factor expression: suggestion of a novel model of embryonic germ cell translocation. Mol Hum Reprod 21(6): 552.

Agger K, Cloos PA, Christensen J, Pasini D, Rose S, Rappsilber J, Issaeva I, Canaani E, Salcini AE, Helin K. 2007. UTX and JMJD3 are histone H3K27 demethylases involved in $\mathrm{HOX}$ gene regulation and development. Nature 449(7163): 731-734.

Agger K, Cloos PA, Rudkjaer L, Williams K, Andersen G, Christensen J, Helin K. 2009. The H3K27me3 demethylase JMJD3 contributes to the activation of the 
INK4A-ARF locus in response to oncogene- and stress-induced senescence. Genes \& development 23(10): 1171-1176.

Aizawa H, Hu SC, Bobb K, Balakrishnan K, Ince G, Gurevich I, Cowan M, Ghosh A. 2004. Dendrite development regulated by CREST, a calcium-regulated transcriptional activator. Science 303(5655): 197-202.

Albert M, Huttner WB. 2018. Epigenetic and Transcriptional Pre-patterning-An Emerging Theme in Cortical Neurogenesis. Frontiers in Neuroscience 12.

Alcamo EA, Chirivella L, Dautzenberg M, Dobreva G, Farinas I, GrosschedI R, McConnell SK. 2008. Satb2 regulates callosal projection neuron identity in the developing cerebral cortex. Neuron 57(3): 364-377.

Alexander JM, Hota SK, He D, Thomas S, Ho L, Pennacchio LA, Bruneau BG. 2015. Brg1 modulates enhancer activation in mesoderm lineage commitment. Development 142(8): 1418-1430.

Alfert A, Moreno N, Kerl K. 2019. The BAF complex in development and disease. Epigenetics Chromatin 12(1): 19.

Alver BH, Kim KH, Lu P, Wang XF, Manchester HE, Wang WS, Haswell JR, Park PJ, Roberts CWM. 2017. The SWI/SNF chromatin remodelling complex is required for maintenance of lineage specific enhancers. Nature Communications 8.

Amaniti EM, Hasenpusch-Theil K, Li ZW, Magnani D, Kessaris N, Mason JO, Theil T. 2013. Gli3 is required in Emx1(+) progenitors for the development of the corpus callosum. Developmental Biology 376(2): 113-124.

Angevine JB, Jr., Sidman RL. 1961. Autoradiographic study of cell migration during histogenesis of cerebral cortex in the mouse. Nature 192: 766-768.

Anton ES, Kreidberg JA, Rakic P. 1999. Distinct functions of alpha3 and alpha(v) integrin receptors in neuronal migration and laminar organization of the cerebral cortex. Neuron 22(2): 277-289.

Anton ES, Marchionni MA, Lee KF, Rakic P. 1997. Role of GGF/neuregulin signaling in interactions between migrating neurons and radial glia in the developing cerebral cortex. Development 124(18): 3501-3510.

Arlotta P, Molyneaux BJ, Chen J, Inoue J, Kominami R, Macklis JD. 2005. Neuronal subtype-specific genes that control corticospinal motor neuron development in vivo. Neuron 45(2): 207-221.

Armentano M, Chou SJ, Tomassy GS, Leingaertner A, O'Leary DDM, Studer M. 2007. COUP-TFI regulates the balance of cortical patterning between frontal/motor and sensory areas. Nature Neuroscience 10(10): 1277-1286.

Ayala R, Shu T, Tsai LH. 2007. Trekking across the brain: the journey of neuronal migration. Cell 128(1): 29-43.

Bachmann C, Nguyen H, Rosenbusch J, Pham L, Rabe T, Patwa M, Sokpor G, Seong RH, Ashery-Padan R, Mansouri A, et al. 2016. mSWI/SNF (BAF) Complexes Are Indispensable for the Neurogenesis and Development of Embryonic Olfactory Epithelium. PLoS Genet 12(9): e1006274.

Balasubramanian R, Bui A, Ding Q, Gan L. 2014. Expression of LIM-homeodomain transcription factors in the developing and mature mouse retina. Gene Expr Patterns 14(1): 1-8.

Barker N, Hurlstone A, Musisi H, Miles A, Bienz M, Clevers H. 2001. The chromatin remodelling factor Brg-1 interacts with beta-catenin to promote target gene activation. Embo Journal 20(17): 4935-4943. 
Barnat M, Le Friec J, Benstaali C, Humbert S. 2017. Huntingtin-Mediated MultipolarBipolar Transition of Newborn Cortical Neurons Is Critical for Their Postnatal Neuronal Morphology. Neuron 93(1): 99-114.

Barutcu AR, Lajoie BR, Fritz AJ, McCord RP, Nickerson JA, van Wijnen AJ, Lian JB, Stein JL, Dekker J, Stein GS, et al. 2016. SMARCA4 regulates gene expression and higher-order chromatin structure in proliferating mammary epithelial cells. Genome Research 26(9): 1188-1201.

Baudet ML, Bellon A, Holt CE. 2013. Role of microRNAs in Semaphorin function and neural circuit formation. Seminars in Cell \& Developmental Biology 24(3): 146155.

Benadiba C, Magnani D, Niquille M, Morle L, Valloton D, Nawabi H, Ait-Lounis A, Otsmane B, Reith W, Theil T, et al. 2012. The ciliogenic transcription factor RFX3 regulates early midline distribution of guidepost neurons required for corpus callosum development. PLoS Genet 8(3): e1002606.

Bentivoglio M, Mazzarello P. 1999. The history of radial glia. Brain Res Bull 49(5): 305-315.

Betizeau M, Cortay V, Patti D, Pfister S, Gautier E, Bellemin-Menard A, Afanassieff M, Huissoud C, Douglas RJ, Kennedy H, et al. 2013. Precursor Diversity and Complexity of Lineage Relationships in the Outer Subventricular Zone of the Primate. Neuron 80(2): 442-457.

Bielle F, Griveau A, Narboux-Nême N, Vigneau S, Sigrist M, Arber S, Wassef M, Pierani A. 2005. Multiple origins of Cajal-Retzius cells at the borders of the developing pallium. Nature Neuroscience 8(8): 1002-1012.

Bischof M, Weider M, Küspert M, Nave K-A, Wegner M. 2015. Brg1-dependent chromatin remodelling is not essentially required during oligodendroglial differentiation. The Journal of neuroscience : the official journal of the Society for Neuroscience 35(1): 21-35.

Bishop KM, Goudreau G, O'Leary DDM. 2000. Regulation of area identity in the mammalian neocortex by Emx2 and Pax6. Science 288(5464): 344-349.

Bocchi R, Egervari K, Carol-Perdiguer L, Viale B, Quairiaux C, De Roo M, Boitard M, Oskouie S, Salmon P, Kiss JZ. 2017. Perturbed Wnt signaling leads to neuronal migration delay, altered interhemispheric connections and impaired social behavior. Nature Communications 8.

Bohmdorfer G, Wierzbicki AT. 2015. Control of Chromatin Structure by Long Noncoding RNA. Trends in cell biology 25(10): 623-632.

Boitard M, Bocchi R, Egervari K, Petrenko V, Viale B, Gremaud S, Zgraggen E, Salmon P, Kiss JZ. 2015. Wnt Signaling Regulates Multipolar-to-Bipolar Transition of Migrating Neurons in the Cerebral Cortex. Cell Reports 10(8): 1349-1361.

Borello U, Pierani A. 2010. Patterning the cerebral cortex: traveling with morphogens. Current Opinion in Genetics \& Development 20(4): 408-415.

Borrell V, Gotz M. 2014. Role of radial glial cells in cerebral cortex folding. Current Opinion in Neurobiology 27: 39-46.

Bossen C, Murre CS, Chang AN, Mansson R, Rodewald HR, Murre C. 2015. The chromatin remodeler Brg1 activates enhancer repertoires to establish B cell identity and modulate cell growth. Nature Immunology 16(7): 775-+.

Britanova O, de Juan Romero C, Cheung A, Kwan KY, Schwark M, Gyorgy A, Vogel T, Akopov S, Mitkovski M, Agoston D, et al. 2008. Satb2 is a postmitotic determinant for upper-layer neuron specification in the neocortex. Neuron 57(3): 378-392. 
Bulchand S, Grove EA, Porter FD, Tole S. 2001. LIM-homeodomain gene Lhx2 regulates the formation of the cortical hem. Mech Dev 100(2): 165-175.

Bulchand S, Subramanian L, Tole S. 2003. Dynamic spatiotemporal expression of LIM genes and cofactors in the embryonic and postnatal cerebral cortex. Dev Dyn 226(3): 460-469.

Bultman S, Gebuhr T, Yee D, La Mantia C, Nicholson J, Gilliam A, Randazzo F, Metzger D, Chambon P, Crabtree G, et al. 2000. A Brg1 null mutation in the mouse reveals functional differences among mammalian SWI/SNF complexes. Molecular Cell 6(6): 1287-1295.

Cairns BR. 1998. Chromatin remodeling machines: similar motors, ulterior motives. Trends in Biochemical Sciences 23(1): 20-25.

Cameron RS, Rakic P. 1991. Glial cell lineage in the cerebral cortex: a review and synthesis. Glia 4(2): 124-137.

Cao R, Wang L, Wang H, Xia L, Erdjument-Bromage H, Tempst P, Jones RS, Zhang Y. 2002. Role of histone H3 lysine 27 methylation in Polycomb-group silencing. Science 298(5595): 1039-1043.

Caronia-Brown G, Yoshida M, Gulden F, Assimacopoulos S, Grove EA. 2014. The cortical hem regulates the size and patterning of neocortex. Development 141(14): 2855-2865.

Chen J, Archer TK. 2005. Regulating SWI/SNF subunit levels via protein-protein interactions and proteasomal degradation: BAF155 and BAF170 limit expression of BAF57. Molecular and cellular biology 25(20): 9016-9027.

Chen T, Dent SY. 2014. Chromatin modifiers and remodellers: regulators of cellular differentiation. Nat Rev Genet 15(2): 93-106.

Chen TD, Wu QW, Zhang Y, Zhang D. 2015. NDUFV2 regulates neuronal migration in the developing cerebral cortex through modulation of the multipolar-bipolar transition. Brain Research 1625: 102-110.

Chenn A, Walsh CA. 2002. Regulation of cerebral cortical size by control of cell cycle exit in neural precursors. Science 297(5580): 365-369.

Chizhikov VV, Lindgren AG, Mishima Y, Roberts RW, Aldinger KA, Miesegaes GR, Currle DS, Monuki ES, Millen KJ. 2010. Lmx1a regulates fates and location of cells originating from the cerebellar rhombic lip and telencephalic cortical hem. Proc Natl Acad Sci U S A 107(23): 10725-10730.

Choi J, Ko M, Jeon S, Jeon Y, Park K, Lee C, Lee H, Seong RH. 2012. The SWI/SNF-like BAF Complex Is Essential for Early B Cell Development. Journal of Immunology 188(8): 3791-3803.

Chou SJ, Perez-Garcia CG, Kroll TT, O'Leary DDM. 2009. Lhx2 specifies regional fate in Emx1 lineage of telencephalic progenitors generating cerebral cortex. Nature Neuroscience 12(11): 1381-1389.

Clapier CR, Iwasa J, Cairns BR, Peterson CL. 2017. Mechanisms of action and regulation of ATP-dependent chromatin-remodelling complexes. Nat Rev Mol Cell Biol 18(7): 407-422.

Clovis YM, Enard W, Marinaro F, Huttner WB, De Pietri Tonelli D. 2012. Convergent repression of Foxp2 3'UTR by miR-9 and miR-132 in embryonic mouse neocortex: implications for radial migration of neurons. Development 139(18): 3332-3342.

Conti L, Pollard SM, Gorba T, Reitano E, Toselli M, Biella G, Sun Y, Sanzone S, Ying QL, Cattaneo E, et al. 2005. Niche-independent symmetrical self-renewal of a mammalian tissue stem cell. PLoS Biol 3(9): e283. 
Coskun V, Tsoa R, Sun YE. 2012. Epigenetic regulation of stem cells differentiating along the neural lineage. Curr Opin Neurobiol 22(5): 762-767.

Crossley PH, Martinez S, Ohkubo Y, Rubenstein JLR. 2001. Coordinate expression of Fgf8, Otx2, Bmp4, and Shh in the rostral prosencephalon during development of the telencephalic and optic vesicles. Neuroscience 108(2): 183-206.

de Melo J, Clark BS, Blackshaw S. 2016. Multiple intrinsic factors act in concert with Lhx2 to direct retinal gliogenesis. Sci Rep 6: 32757.

de Melo J, Clark BS, Venkataraman A, Shiau F, Zibetti C, Blackshaw S. 2018. Ldb1 - and Rnf12-dependent regulation of Lhx2 controls the relative balance between neurogenesis and gliogenesis in the retina. Development 145(9).

De Santa F, Totaro MG, Prosperini E, Notarbartolo S, Testa G, Natoli G. 2007. The histone $\mathrm{H} 3$ lysine-27 demethylase Jmjd3 links inflammation to inhibition of polycomb-mediated gene silencing. Cell 130(6): 1083-1094.

Dehay C, Kennedy H, Kosik KS. 2015. The Outer Subventricular Zone and PrimateSpecific Cortical Complexification. Neuron 85(4): 683-694.

Delaloy C, Liu L, Lee JA, Su H, Shen F, Yang GY, Young WL, Ivey KN, Gao FB. 2010. MicroRNA-9 coordinates proliferation and migration of human embryonic stem cell-derived neural progenitors. Cell Stem Cell 6(4): 323-335.

Desai R, Sarpal R, Ishiyama N, Pellikka M, Ikura M, Tepass U. 2013. Monomeric alpha-catenin links cadherin to the actin cytoskeleton. Nat Cell Biol 15(3): 261273.

Drees F, Pokutta S, Yamada S, Nelson WJ, Weis WI. 2005. Alpha-catenin is a molecular switch that binds E-cadherin-beta-catenin and regulates actinfilament assembly. Cell 123(5): 903-915.

Elias LAB, Wang DD, Kriegstein AR. 2007. Gap junction adhesion is necessary for radial migration in the neocortex. Nature 448(7156): 901-U903.

Elsen GE, Bedogni F, Hodge RD, Bammler TK, MacDonald JW, Lindtner S, Rubenstein JLR, Hevner RF. 2018. The Epigenetic Factor Landscape of Developing Neocortex Is Regulated by Transcription Factors Pax6 -> Tbr2 -> Tbr1. Frontiers in Neuroscience 12.

Emami KH, Nguyen C, Ma H, Kim DH, Jeong KW, Eguchi M, Moon RT, Teo JL, Kim HY, Moon SH, et al. 2004. A small molecule inhibitor of betacatenin/CREB-binding protein transcription [corrected]. Proc Natl Acad Sci U S A 101(34): 12682-12687.

Erhardt S, Su IH, Schneider R, Barton S, Bannister AJ, Perez-Burgos L, Jenuwein T, Kouzarides T, Tarakhovsky A, Surani MA. 2003. Consequences of the depletion of zygotic and embryonic enhancer of zeste 2 during preimplantation mouse development. Development 130(18): 4235-4248.

Evsyukova I, Plestant C, Anton ES. 2013. Integrative mechanisms of oriented neuronal migration in the developing brain. Annu Rev Cell Dev Biol 29: 299353.

Fernandez V, Llinares-Benadero C, Borrell V. 2016. Cerebral cortex expansion and folding: what have we learned? Embo Journal 35(10): 1021-1044.

Filatova A, Rey LK, Lechler MB, Schaper J, Hempel M, Posmyk R, Szczaluba K, Santen GWE, Wieczorek D, Nuber UA. 2019. Mutations in SMARCB1 and in other Coffin-Siris syndrome genes lead to various brain midline defects. Nature Communications 10.

Florio M, Huttner WB. 2014. Neural progenitors, neurogenesis and the evolution of the neocortex. Development 141(11): 2182-2194. 
Fode C, Ma Q, Casarosa S, Ang SL, Anderson DJ, Guillemot F. 2000. A role for neural determination genes in specifying the dorsoventral identity of telencephalic neurons. Genes Dev 14(1): 67-80.

Folgueras AR, Guo X, Pasolli HA, Stokes N, Polak L, Zheng D, Fuchs E. 2013. Architectural niche organization by LHX2 is linked to hair follicle stem cell function. Cell Stem Cell 13(3): 314-327.

Forster E, Zhao S, Frotscher M. 2006. Laminating the hippocampus. Nat Rev Neurosci 7(4): 259-267.

Franco SJ, Gil-Sanz C, Martinez-Garay I, Espinosa A, Harkins-Perry SR, Ramos C, Muller U. 2012. Fate-Restricted Neural Progenitors in the Mammalian Cerebral Cortex. Science 337(6095): 746-749.

Frantz GD, Weimann JM, Levin ME, Mcconnell SK. 1994. Otx1 and Otx2 Define Layers and Regions in Developing Cerebral-Cortex and Cerebellum. Journal of Neuroscience 14(10): 5725-5740.

Freeman MR. 2010. Specification and morphogenesis of astrocytes. Science 330(6005): 774-778.

Friocourt F, Chedotal A. 2017. The Robo3 receptor, a key player in the development, evolution, and function of commissural systems. Dev Neurobiol 77(7): 876-890.

Frotscher M, Chai XJ, Bock HH, Haas CA, Forster E, Zhao ST. 2009. Role of Reelin in the development and maintenance of cortical lamination. Journal of Neural Transmission 116(11): 1451-1455.

Fuentes P, Canovas J, Berndt FA, Noctor SC, Kukuljan M. 2012. CoREST/LSD1 Control the Development of Pyramidal Cortical Neurons. Cerebral Cortex 22(6): 1431-1441.

Furuta Y, Piston DW, Hogan BLM. 1997. Bone morphogenetic proteins (BMPs) as regulators of dorsal forebrain development. Development 124(11): 2203-2212.

Gage FH. 2000. Mammalian neural stem cells. Science 287(5457): 1433-1438.

Galceran J, Miyashita-Lin EM, Devaney E, Rubenstein JLR, GrosschedI R. 2000. Hippocampus development and generation of dentate gyrus granule cells is regulated by LEF1. Development 127(3): 469-482.

Gallo V, Deneen B. 2014. Glial development: the crossroads of regeneration and repair in the CNS. Neuron 83(2): 283-308.

Gaspard N, Bouschet T, Hourez R, Dimidschstein J, Naeije G, van den Ameele J, Espuny-Camacho I, Herpoel A, Passante L, Schiffmann SN, et al. 2008. An intrinsic mechanism of corticogenesis from embryonic stem cells. Nature 455(7211): 351-U310.

Gitton Y, Cohen-Tannoudji M, Wassef M. 1999. Specification of somatosensory area identity in cortical explants. J Neurosci 19(12): 4889-4898.

Godbole G, Roy A, Shetty AS, Tole S. 2017. Novel functions of LHX2 and PAX6 in the developing telencephalon revealed upon combined loss of both genes. Neural Development 12.

Godbole G, Shetty AS, Roy A, D'Souza L, Chen B, Miyoshi G, Fishell G, Tole S. 2018. Hierarchical genetic interactions between FOXG1 and LHX2 regulate the formation of the cortical hem in the developing telencephalon. Development 145(1).

Goebbels S, Bormuth I, Bode U, Hermanson O, Schwab MH, Nave KA. 2006. Genetic targeting of principal neurons in neocortex and hippocampus of NEXCre mice. Genesis 44(12): 611-621.

Goldberg AD, Allis CD, Bernstein E. 2007. Epigenetics: a landscape takes shape. Cell 128(4): 635-638. 
Gong F, Fahy D, Smerdon MJ. 2006. Rad4-Rad23 interaction with SWI/SNF links ATP-dependent chromatin remodeling with nucleotide excision repair. Nature Structural \& Molecular Biology 13(10): 902-907.

Gorski JA, Talley T, Qiu MS, Puelles L, Rubenstein JLR, Jones KR. 2002. Cortical excitatory neurons and glia, but not GABAergic neurons, are produced in the Emx1-expressing lineage. Journal of Neuroscience 22(15): 6309-6314.

Gotz M, Huttner WB. 2005. The cell biology of neurogenesis. Nat Rev Mol Cell Biol 6(10): 777-788.

Greig LC, Woodworth MB, Galazo MJ, Padmanabhan H, Macklis JD. 2013. Molecular logic of neocortical projection neuron specification, development and diversity. Nat Rev Neurosci 14(11): 755-769.

Grove EA, Tole S, Limon J, Yip LW, Ragsdale CW. 1998. The hem of the embryonic cerebral cortex is defined by the expression of multiple Wnt genes and is compromised in Gli3-deficient mice. Development 125(12): 2315-2325.

Guillemot F. 2005. Cellular and molecular control of neurogenesis in the mammalian telencephalon. Current Opinion in Cell Biology 17(6): 639-647.

Guillemot F. 2007. Cell fate specification in the mammalian telencephalon. Prog Neurobiol 83(1): 37-52.

Guo H, Hong S, Jin XL, Chen RS, Avasthi PP, Tu YT, Ivanco TL, Li Y. 2000. Specificity and efficiency of Cre-mediated recombination in Emx1-Cre knock-in mice. Biochem Biophys Res Commun 273(2): 661-665.

Guo Y, He X, Zhao L, Liu L, Song H, Wang X, Xu J, Ju X, Guo W, Zhu X. 2017. G $\beta 2$ Regulates the Multipolar-Bipolar Transition of Newborn Neurons in the Developing Neocortex. Cerebral Cortex 27(6): 3414-3426.

Gutierrez J, Paredes R, Cruzat F, Hill DA, van Wijnen AJ, Lian JB, Stein GS, Stein JL, Imbalzano AN, Montecino M. 2007. Chromatin remodeling by SWI/SNF results in nucleosome mobilization to preferential positions in the rat osteocalcin gene promoter. Journal of Biological Chemistry 282(13): 9445-9457.

Hakanen J, Ruiz-Reig N, Tissir F. 2019. Linking Cell Polarity to Cortical Development and Malformations. Frontiers in Cellular Neuroscience 13.

Hamasaki T, Leingartner A, Ringstedt T, O'Leary DD. 2004. EMX2 regulates sizes and positioning of the primary sensory and motor areas in neocortex by direct specification of cortical progenitors. Neuron 43(3): 359-372.

Han WQ, Kwan KY, Shim S, Lam MMS, Shin Y, Xu XM, Zhu Y, Li MF, Sestan N. 2011. TBR1 directly represses Fezf2 to control the laminar origin and development of the corticospinal tract. Proceedings of the National Academy of Sciences of the United States of America 108(7): 3041-3046.

Hanashima C, Fernandes M, Hebert JM, Fishell G. 2007. The role of Foxg1 and dorsal midline signaling in the generation of Cajal-Retzius subtypes. J Neurosci 27(41): 11103-11111.

Hand R, Bortone D, Mattar P, Nguyen L, Heng JI, Guerrier S, Boutt E, Peters E, Barnes AP, Parras C, et al. 2005. Phosphorylation of Neurogenin2 specifies the migration properties and the dendritic morphology of pyramidal neurons in the neocortex. Neuron 48(1): 45-62.

Hansen DV, Lui JH, Parker PRL, Kriegstein AR. 2010. Neurogenic radial glia in the outer subventricular zone of human neocortex. Nature 464(7288): 554-U110.

Hara R, Sancar A. 2002. The SWI/SNF chromatin-remodeling factor stimulates repair by human excision nuclease in the mononucleosome core particle. Molecular and cellular biology 22(19): 6779-6787. 
Harb K, Magrinelli E, Nicolas CS, Lukianets N, Frangeul L, Pietri M, Sun T, Sandoz G, Grammont F, Jabaudon D, et al. 2016. Area-specific development of distinct projection neuron subclasses is regulated by postnatal epigenetic modifications. Elife 5.

Harrison-Uy SJ, Pleasure SJ. 2012. Wnt Signaling and Forebrain Development. Cold Spring Harbor Perspectives in Biology 4(7).

Hartfuss E, Galli R, Heins N, Gotz M. 2001. Characterization of CNS precursor subtypes and radial glia. Dev Biol 229(1): 15-30.

Hasenpusch-Theil K, Watson JA, Theil T. 2017. Direct Interactions Between Gli3, Wnt8b, and Fgfs Underlie Patterning of the Dorsal Telencephalon. Cerebral Cortex 27(2): 1137-1148.

Hashimoto-Torii K, Torii M, Sarkisian MR, Bartley CM, Shen J, Radtke F, Gridley T, Sestan N, Rakic P. 2008. Interaction between Reelin and Notch Signaling Regulates Neuronal Migration in the Cerebral Cortex. Neuron 60(2): 273-284.

Hatanaka Y, Yamauchi K. 2013. Excitatory cortical neurons with multipolar shape establish neuronal polarity by forming a tangentially oriented axon in the intermediate zone. Cereb Cortex 23(1): 105-113.

Haubensak W, Attardo A, Denk W, Huttner WB. 2004. Neurons arise in the basal neuroepithelium of the early mammalian telencephalon: a major site of neurogenesis. Proc Natl Acad Sci U S A 101(9): 3196-3201.

Hawthorne AL, Wylie CJ, Landmesser LT, Deneris ES, Silver J. 2010. Serotonergic Neurons Migrate Radially through the Neuroepithelium by Dynamin-Mediated Somal Translocation. Journal of Neuroscience 30(2): 420-430.

Hayashi S, McMahon AP. 2002. Efficient recombination in diverse tissues by a tamoxifen-inducible form of Cre: a tool for temporally regulated gene activation/inactivation in the mouse. Dev Biol 244(2): 305-318.

Heard E, Martienssen RA. 2014. Transgenerational epigenetic inheritance: myths and mechanisms. Cell 157(1): 95-109.

Hébert JM, Fishell G. 2008. The genetics of early telencephalon patterning: some assembly required. Nature reviews. Neuroscience 9(9): 678-685.

Hebert JM, McConnell SK. 2000. Targeting of cre to the Foxg1 (BF-1) locus mediates loxP recombination in the telencephalon and other developing head structures. Dev Biol 222(2): 296-306.

Helming KC, Wang $X$, Roberts CW. 2014. Vulnerabilities of mutant SWI/SNF complexes in cancer. Cancer Cell 26(3): 309-317.

Heng JI, Moonen G, Nguyen L. 2007. Neurotransmitters regulate cell migration in the telencephalon. Eur J Neurosci 26(3): 537-546.

Herbert J, Wilcox JN, Pham KT, Fremeau RT, Jr., Zeviani M, Dwork A, Soprano DR, Makover A, Goodman DS, Zimmerman EA, et al. 1986. Transthyretin: a choroid plexus-specific transport protein in human brain. The $1986 \mathrm{~S}$. Weir Mitchell award. Neurology 36(7): 900-911.

Hevner RF, Shi LM, Justice N, Hsueh TP, Sheng M, Smiga S, Bulfone A, Goffinet AM, Campagnoni AT, Rubenstein JLR. 2001. Tbr1 regulates differentiation of the preplate and layer 6. Neuron 29(2): 353-366.

Hirabayashi Y, Gotoh Y. 2010. Epigenetic control of neural precursor cell fate during development. Nat Rev Neurosci 11(6): 377-388.

Hirota J, Mombaerts P. 2004. The LIM-homeodomain protein Lhx2 is required for complete development of mouse olfactory sensory neurons. Proc Natl Acad Sci U S A 101(23): 8751-8755. 
Hirschhorn JN, Brown SA, Clark CD, Winston F. 1992. Evidence that SNF2/SWI2 and SNF5 activate transcription in yeast by altering chromatin structure. Genes \& development 6(12A): 2288-2298.

Ho L, Crabtree GR. 2011. Chromatin remodelling during development. Nature 463(7280): 474-484.

Ho L, Jothi R, Ronan JL, Cui K, Zhao K, Crabtree GR. 2009a. An embryonic stem cell chromatin remodeling complex, esBAF, is an essential component of the core pluripotency transcriptional network. Proc Natl Acad Sci U S A 106(13): 5187-5191.

Ho L, Miller EL, Ronan JL, Ho WQ, Jothi R, Crabtree GR. 2011. esBAF facilitates pluripotency by conditioning the genome for LIF/STAT3 signalling and by regulating polycomb function. Nature Cell Biology 13(8): 903-913.

Ho L, Ronan JL, Wu J, Staahl BT, Chen L, Kuo A, Lessard J, Nesvizhskii Al, Ranish J, Crabtree GR. 2009b. An embryonic stem cell chromatin remodeling complex, esBAF, is essential for embryonic stem cell self-renewal and pluripotency. Proceedings of the National Academy of Sciences of the United States of America 106(13): 5181-5186.

Holdhof D, Schoof M, Hellwig M, Holdhof NH, Niesen J, Schuller U. 2019. hGFAPPositive Stem Cells Depend on Brg1 for Proper Formation of Cerebral and Cerebellar Structures. Cereb Cortex.

Hong S, Cho YW, Yu LR, Yu H, Veenstra TD, Ge K. 2007. Identification of JmjC domain-containing UTX and JMJD3 as histone H3 lysine 27 demethylases. Proceedings of the National Academy of Sciences of the United States of America 104(47): 18439-18444.

Hou PS, Chuang CY, Kao CF, Chou SJ, Stone L, Ho HN, Chien CL, Kuo HC. 2013. LHX2 regulates the neural differentiation of human embryonic stem cells via transcriptional modulation of PAX6 and CER1. Nucleic Acids Res 41(16): 77537770 .

Hsu LC, Nam S, Cui Y, Chang CP, Wang CF, Kuo HC, Touboul JD, Chou SJ. 2015. Lhx2 regulates the timing of beta-catenin-dependent cortical neurogenesis. Proc Natl Acad Sci U S A 112(39): 12199-12204.

Hu GQ, Schones DE, Cui KR, Ybarra R, Northrup D, Tang QS, Gattinoni L, Restifo NP, Huang SM, Zhao KJ. 2011. Regulation of nucleosome landscape and transcription factor targeting at tissue-specific enhancers by BRG1. Genome Research 21(10): 1650-1658.

Huang YX, Li GH, An L, Fan YL, Cheng XR, Li XZ, Yin YP, Cong RH, Chen SL, Zhao ST. 2017. Fyn Regulates Multipolar-Bipolar Transition and Neurite Morphogenesis of Migrating Neurons in the Developing Neocortex. Neuroscience 352: 39-51.

Huh SO, Hatini V, Marcus RC, Li SC, Lai E. 1999. Dorsal-ventral patterning defects in the eye of BF-1-deficient mice associated with a restricted loss of shh expression. Developmental Biology 211(1): 53-63.

Inoue M, Iwai R, Tabata H, Konno D, Komabayashi-Suzuki M, Watanabe C, Iwanari H, Mochizuki Y, Hamakubo T, Matsuzaki F, et al. 2017. Prdm16 is crucial for progression of the multipolar phase during neural differentiation of the developing neocortex. Development 144(3): 385-399.

Inoue M, Kuroda T, Honda A, Komabayashi-Suzuki M, Komai T, Shinkai Y, Mizutani KI. 2014. Prdm8 Regulates the Morphological Transition at Multipolar Phase during Neocortical Development. Plos One 9(1). 
Iwai R, Tabata H, Inoue M, Nomura K, Okamoto T, Ichihashi M, Nagata K, Mizutani K. 2018. A Prdm8 target gene Ebf3 regulates multipolar-to-bipolar transition in migrating neocortical cells. Biochemical and Biophysical Research Communications 495(1): 388-394.

Jepsen K, Solum D, Zhou T, McEvilly RJ, Kim HJ, Glass CK, Hermanson O, Rosenfeld MG. 2007. SMRT-mediated repression of an H3K27 demethylase in progression from neural stem cell to neuron. Nature 450(7168): 415-419.

Jinnou H, Sawada M, Kawase K, Kaneko N, Herranz-Perez V, Miyamoto T, Kawaue T, Miyata T, Tabata Y, Akaike T, et al. 2018. Radial Glial Fibers Promote Neuronal Migration and Functional Recovery after Neonatal Brain Injury. Cell Stem Cell 22(1): 128-137 e129.

John A, Brylka H, Wiegreffe C, Simon R, Liu P, Jüttner R, Crenshaw EB, 3rd, Luyten FP, Jenkins NA, Copeland NG, et al. 2012. Bcl11a is required for neuronal morphogenesis and sensory circuit formation in dorsal spinal cord development. Development (Cambridge, England) 139(10): 1831-1841.

Juliandi B, Abematsu M, Nakashima K. 2010. Chromatin remodeling in neural stem cell differentiation. Curr Opin Neurobiol 20(4): 408-415.

Jung I, Sohn DH, Choi J, Kim JM, Jeon S, Seol JH, Seong RH. 2012. SRG3/mBAF155 stabilizes the SWI/SNF-like BAF complex by blocking CHFR mediated ubiquitination and degradation of its major components. Biochem Biophys Res Commun 418(3): 512-517.

Kadoch C, Copeland RA, Keilhack H. 2016. PRC2 and SWI/SNF Chromatin Remodeling Complexes in Health and Disease. Biochemistry 55(11): 16001614.

Kadoch C, Hargreaves DC, Hodges C, Elias L, Ho L, Ranish J, Crabtree GR. 2013. Proteomic and bioinformatic analysis of mammalian SWI/SNF complexes identifies extensive roles in human malignancy. Nature genetics 45(6): 592-601.

Kadoch C, Williams RT, Calarco JP, Miller EL, Weber CM, Braun SM, Pulice JL, Chory EJ, Crabtree GR. 2017. Dynamics of BAF-Polycomb complex opposition on heterochromatin in normal and oncogenic states. Nature genetics 49(2): 213-222.

Kammandel B, Chowdhury K, Stoykova A, Aparicio S, Brenner S, Gruss P. 1999. Distinct cis-essential modules direct the time-space pattern of the Pax6 gene activity. Dev Biol 205(1): 79-97.

Kawauchi T, Sekine K, Shikanai M, Chihama K, Tomita K, Kubo K, Nakajima K, Nabeshima Y, Hoshino M. 2010. Rab GTPases-Dependent Endocytic Pathways Regulate Neuronal Migration and Maturation through N-Cadherin Trafficking. Neuron 67(4): 588-602.

Kelava I, Reillo I, Murayama AY, Kalinka AT, Stenzel D, Tomancak P, Matsuzaki F, Lebrand C, Sasaki E, Schwamborn JC, et al. 2012. Abundant Occurrence of Basal Radial Glia in the Subventricular Zone of Embryonic Neocortex of a Lissencephalic Primate, the Common Marmoset Callithrix jacchus. Cerebral Cortex 22(2): 469-481.

Kiecker C, Lumsden A. 2012. The role of organizers in patterning the nervous system. Annual Review of Neuroscience 35: 347-367.

Kim JK, Huh SO, Choi H, Lee KS, Shin D, Lee C, Nam JS, Kim H, Chung H, Lee HW, et al. 2001. Srg3, a mouse homolog of yeast SWI3, is essential for early embryogenesis and involved in brain development. Molecular and cellular biology 21(22): 7787-7795. 
Kimura J, Suda Y, Kurokawa D, Hossain ZM, Nakamura M, Takahashi M, Hara A, Aizawa S. 2005. Emx2 and Pax6 function in cooperation with Otx2 and Otx1 to develop caudal forebrain primordium that includes future archipallium. Journal of Neuroscience 25(21): 5097-5108.

Ko M, Sohn DH, Chung H, Seong RH. 2008. Chromatin remodeling, development and disease. Mutation research 647(1-2): 59-67.

Kojima Y, Tam OH, Tam PP. 2014. Timing of developmental events in the early mouse embryo. Seminars in cell \& developmental biology 34: 65-75.

Koleske AJ. 2013. Molecular mechanisms of dendrite stability. Nat Rev Neurosci 14(8): 536-550.

Kolterud A, Alenius M, Carlsson L, Bohm S. 2004. The Lim homeobox gene Lhx2 is required for olfactory sensory neuron identity. Development 131(21): 53195326.

Kowalczyk T, Pontious A, Englund C, Daza RAM, Bedogni F, Hodge R, Attardo A, Bell C, Huttner WB, Hevner RF. 2009. Intermediate Neuronal Progenitors (Basal Progenitors) Produce Pyramidal-Projection Neurons for All Layers of Cerebral Cortex. Cerebral Cortex 19(10): 2439-2450.

Kriegstein A, Alvarez-Buylla A. 2009. The glial nature of embryonic and adult neural stem cells. Annu Rev Neurosci 32: 149-184.

Kriegstein AR, Noctor SC. 2004. Patterns of neuronal migration in the embryonic cortex. Trends in Neurosciences 27(7): 392-399.

Kurabayashi N, Tanaka A, Nguyen MD, Sanada K. 2018. The LPA-LPA4 axis is required for establishment of bipolar morphology and radial migration of newborn cortical neurons. Development 145(17).

Kuschel S, Ruther U, Theil T. 2003. A disrupted balance between Bmp/Wnt and Fgf signaling underlies the ventralization of the Gli3 mutant telencephalon. Developmental Biology 260(2): 484-495.

Kwan KY, Lam MM, Krsnik Z, Kawasawa YI, Lefebvre V, Sestan N. 2008. SOX5 postmitotically regulates migration, postmigratory differentiation, and projections of subplate and deep-layer neocortical neurons. Proc Natl Acad Sci U S A 105(41): 16021-16026.

Kwan KY, Sestan N, Anton ES. 2012. Transcriptional co-regulation of neuronal migration and laminar identity in the neocortex. Development 139(9): 15351546.

La Fata G, Gartner A, Dominguez-Iturza N, Dresselaers T, Dawitz J, Poorthuis RB, Averna M, Himmelreich U, Meredith RM, Achsel T, et al. 2014. FMRP regulates multipolar to bipolar transition affecting neuronal migration and cortical circuitry. Nature Neuroscience 17(12): 1693-1700.

Lai T, Jabaudon D, Molyneaux BJ, Azim E, Arlotta P, Menezes JRL, Macklis JD. 2008. SOX5 controls the sequential generation of distinct corticofugal neuron subtypes. Neuron 57(2): 232-247.

Lamba DA, Hayes S, Karl MO, Reh T. 2008. Baf60c is a component of the neural progenitor-specific BAF complex in developing retina. Developmental dynamics : an official publication of the American Association of Anatomists 237(10): 3016-3023.

Lan F, Bayliss PE, Rinn JL, Whetstine JR, Wang JK, Chen S, Iwase S, Alpatov R, Issaeva I, Canaani E, et al. 2007. A histone H3 lysine 27 demethylase regulates animal posterior development. Nature 449(7163): 689-694. 
Laurent BC, Treich I, Carlson M. 1993. The yeast SNF2/SWI2 protein has DNAstimulated ATPase activity required for transcriptional activation. Genes \& development 7(4): 583-591.

Lavado A, He Y, Pare J, Neale G, Olson EN, Giovannini M, Cao X. 2013. Tumor suppressor Nf2 limits expansion of the neural progenitor pool by inhibiting Yap/Taz transcriptional coactivators. Development 140(16): 3323-3334.

Lebensohn AM, Rohatgi R. 2018. R-spondins can potentiate WNT signaling without LGRs. Elife 7.

Lee MG, Villa R, Trojer P, Norman J, Yan KP, Reinberg D, Di Croce L, Shiekhattar R. 2007. Demethylation of H3K27 regulates polycomb recruitment and H2A ubiquitination. Science 318(5849): 447-450.

Lee SM, Tole S, Grove E, McMahon AP. 2000. A local Wnt-3a signal is required for development of the mammalian hippocampus. Development 127(3): 457-467.

Lessard J, Wu JI, Ranish JA, Wan M, Winslow MM, Staahl BT, Wu H, Aebersold R, Graef IA, Crabtree GR. 2007. An essential switch in subunit composition of a chromatin remodeling complex during neural development. Neuron 55(2): 201-215.

Levitt P, Barbe MF, Eagleson KL. 1997. Patterning and specification of the cerebral cortex. Annual Review of Neuroscience 20: 1-24.

Lewitus E, Kelava I, Huttner WB. 2013. Conical expansion of the outer subventricular zone and the role of neocortical folding in evolution and development. Front Hum Neurosci 7: 424.

Li GN, Pleasure SJ. 2005. Morphogenesis of the dentate gyrus: What we are learning from mouse mutants. Developmental Neuroscience 27(2-4): 93-99.

Li H, Bishop KM, O'Leary DDM. 2006. Potential target genes of EMX2 include Odz/Ten-M and other gene families with implications for cortical patterning. Molecular and Cellular Neuroscience 33(2): 136-149.

Lillien L, Gulacsi A. 2006. Environmental signals elicit multiple responses in dorsal telencephalic progenitors by threshold-dependent mechanisms. Cereb Cortex 16 Suppl 1: i74-81.

Liu B, Xiao H, Zhao C. 2018. Forced Expression of Foxg1 in the Cortical Hem Leads to the Transformation of Cajal-Retzius Cells into Dentate Granule Neurons. $J$ Dev Biol 6(3).

Louhivuori LM, Turunen PM, Louhivuori V, Yellapragada V, Nordstrom T, Uhlen P, Akerman KE. 2018. Regulation of radial glial process growth by glutamate via mGluR5/TRPC3 and neuregulin/ErbB4. Glia 66(1): 94-107.

Louvi A, Yoshida M, Grove EA. 2007. The derivatives of the Wnt3a lineage in the central nervous system. Journal of Comparative Neurology 504(5): 550-569.

Lu C, Allis CD. 2017. SWI/SNF complex in cancer. Nature genetics 49(2): 178-179.

Lui JH, Hansen DV, Kriegstein AR. 2011. Development and evolution of the human neocortex. Cell 146(1): 18-36.

Machon O, Backman M, Machonova O, Kozmik Z, Vacik T, Andersen L, Krauss S. 2007. A dynamic gradient of Wnt signaling controls initiation of neurogenesis in the mammalian cortex and cellular specification in the hippocampus. Developmental Biology 311(1): 223-237.

Magnani D, Hasenpusch-Theil K, Benadiba C, Yu T, Basson MA, Price DJ, Lebrand C, Theil T. 2014. Gli3 Controls Corpus Callosum Formation by Positioning Midline Guideposts During Telencephalic Patterning. Cerebral Cortex 24(1): 186-198. 
Malatesta P, Hack MA, Hartfuss E, Kettenmann H, Klinkert W, Kirchhoff F, Gotz M. 2003. Neuronal or glial progeny: regional differences in radial glia fate. Neuron 37(5): 751-764.

Mallamaci A, Muzio L, Chan CH, Parnavelas J, Boncinelli E. 2000. Area identity shifts in the early cerebral cortex of Emx2-/- mutant mice. Nature Neuroscience 3(7): 679-686.

Mallamaci A, Stoykova A. 2006. Gene networks controlling early cerebral cortex arealization. Eur J Neurosci 23(4): 847-856.

Mangale VS, Hirokawa KE, Satyaki PR, Gokulchandran N, Chikbire S, Subramanian L, Shetty AS, Martynoga B, Paul J, Mai MV, et al. 2008a. Lhx2 selector activity specifies cortical identity and suppresses hippocampal organizer fate. Science 319(5861): 304-309.

Mangale VS, Hirokawa KE, Satyaki PRV, Gokulchandran N, Chikbire S, Subramanian L, Shetty AS, Martynoga B, Paul J, Mai MV, et al. 2008b. Lhx2 selector activity specifies cortical identity and suppresses hippocampal organizer fate. Science 319(5861): 304-309.

Marin-Padilla M. 1978. Dual origin of the mammalian neocortex and evolution of the cortical plate. Anat Embryol (Berl) 152(2): 109-126.

Marin O, Rubenstein JL. 2003. Cell migration in the forebrain. Annu Rev Neurosci 26: 441-483.

Marin O, Valiente M, Ge X, Tsai LH. 2010. Guiding neuronal cell migrations. Cold Spring Harb Perspect Biol 2(2): a001834.

Martinez-Cerdeno V, Noctor SC. 2016. Cortical evolution 2015: Discussion of neural progenitor cell nomenclature. Journal of Comparative Neurology 524(3): 704709.

Martinez-Chavez E, Scheerer C, Wizenmann A, Blaess S. 2018. The zinc-finger transcription factor GLI3 is a regulator of precerebellar neuronal migration. Development 145(24).

Martynoga B, Morrison H, Price DJ, Mason JO. 2005. Foxg1 is required for specification of ventral telencephalon and region-specific regulation of dorsal telencephalic precursor proliferation and apoptosis. Developmental Biology 283(1): 113-127.

Masliah-Planchon J, Bieche I, Guinebretiere JM, Bourdeaut F, Delattre O. 2015. SWI/SNF chromatin remodeling and human malignancies. Annual review of pathology 10: 145-171.

Matsumoto S, Banine F, Struve J, Xing RB, Adams C, Liu Y, Metzger D, Chambon P, Rao MS, Sherman LS. 2006. Brg1 is required for murine neural stem cell maintenance and gliogenesis. Developmental Biology 289(2): 372-383.

McEvilly RJ, de Diaz MO, Schonemann MD, Hooshmand F, Rosenfeld MG. 2002. Transcriptional regulation of cortical neuron migration by POU domain factors. Science 295(5559): 1528-1532.

McLoughlin HS, Fineberg SK, Ghosh LL, Tecedor L, Davidson BL. 2012. Dicer Is Required for Proliferation, Viability, Migration and Differentiation in Corticoneurogenesis. Neuroscience 223: 285-295.

Meyer G, Perez-Garcia CG, Abraham H, Caput D. 2002. Expression of p73 and Reelin in the developing human cortex. Journal of Neuroscience 22(12): 49734986.

Meyer G, Socorro AC, Garcia CGP, Millan LM, Walker N, Caput D. 2004. Developmental roles of p73 in Cajal-Retzius cells and cortical patterning. Journal of Neuroscience 24(44): 9878-9887. 
Miyata T, Kawaguchi A, Okano H, Ogawa M. 2001. Asymmetric inheritance of radial glial fibers by cortical neurons. Neuron 31(5): 727-741.

Miyata T, Kawaguchi A, Saito K, Kawano M, Muto T, Ogawa M. 2004. Asymmetric production of surface-dividing and non-surface-dividing cortical progenitor cells. Development 131(13): 3133-3145.

Mizutani KI. 2018. Physiological significance of multipolar cells generated from neural stem cells and progenitors for the establishment of neocortical cytoarchitecture. Genes to Cells 23(1): 6-15.

Mohn F, Weber M, Rebhan M, Roloff TC, Richter J, Stadler MB, Bibel M, Schubeler D. 2008. Lineage-specific polycomb targets and de novo DNA methylation define restriction and potential of neuronal progenitors. Molecular Cell 30(6): 755-766.

Mollgoard K, Saunders NR. 1975. Complex tight junctions of epithelial and of endothelial cells in early foetal brain. J Neurocytol 4(4): 453-468.

Molliver ME, Kostovic I, van der Loos H. 1973. The development of synapses in cerebral cortex of the human fetus. Brain Research 50(2): 403-407.

Molyneaux BJ, Arlotta P, Hirata T, Hibi M, Macklis JD. 2005. Fezl is required for the birth and specification of corticospinal motor neurons. Neuron 47(6): 817-831.

Molyneaux BJ, Arlotta P, Menezes JR, Macklis JD. 2007. Neuronal subtype specification in the cerebral cortex. Nat Rev Neurosci 8(6): 427-437.

Monuki ES, Porter FD, Walsh CA. 2001. Patterning of the dorsal telencephalon and cerebral cortex by a roof plate-Lhx2 pathway. Neuron 32(4): 591-604.

Monuki ES, Walsh CA. 2001. Mechanisms of cerebral cortical patterning in mice and humans. Nature Neuroscience 4 Suppl: 1199-1206.

Mountcastle VB. 1997. The columnar organization of the neocortex. Brain 120 ( Pt 4): 701-722.

MuhChyi C, Juliandi B, Matsuda T, Nakashima K. 2013. Epigenetic regulation of neural stem cell fate during corticogenesis. International journal of developmental neuroscience : the official journal of the International Society for Developmental Neuroscience 31(6): 424-433.

Mutch CA, Schulte JD, Olson E, Chenn A. 2010. Beta-catenin signaling negatively regulates intermediate progenitor population numbers in the developing cortex. Plos One 5(8): e12376.

Muzio L, DiBenedetto B, Stoykova A, Boncinelli E, Gruss P, Mallamaci A. 2002. Emx2 and Pax6 control regionalization of the pre-neuronogenic cortical primordium. Cerebral Cortex 12(2): 129-139.

Muzio L, Mallamaci A. 2003. Emx1, Emx2 and Pax6 in specification, regionalization and arealization of the cerebral cortex. Cerebral Cortex 13(6): 641-647.

Muzio L, Mallamaci A. 2005. Foxg1 confines Cajal-Retzius neuronogenesis and hippocampal morphogenesis to the dorsomedial pallium. Journal of Neuroscience 25(17): 4435-4441.

Nadarajah B, Brunstrom JE, Grutzendler J, Wong ROL, Pearlman AL. 2001. Two modes of radial migration in early development of the cerebral cortex. Nature Neuroscience 4(2): 143-150.

Namba T, Kibe Y, Funahashi Y, Nakamuta S, Takano T, Ueno T, Shimada A, Kozawa S, Okamoto M, Shimoda Y, et al. 2014. Pioneering axons regulate neuronal polarization in the developing cerebral cortex. Neuron 81(4): 814-829.

Narayanan R, Pham L, Kerimoglu C, Watanabe T, Castro Hernandez R, Sokpor G, Ulmke PA, Kiszka KA, Tonchev AB, Rosenbusch J, et al. 2018. 
Chromatin Remodeling BAF155 Subunit Regulates the Genesis of Basal Progenitors in Developing Cortex. iScience 4: 109-126.

Narayanan R, Pirouz M, Kerimoglu C, Pham L, Wagener RJ, Kiszka KA, Rosenbusch J, Seong RH, Kessel M, Fischer A, et al. 2015. Loss of BAF (mSWI/SNF) Complexes Causes Global Transcriptional and Chromatin State Changes in Forebrain Development. Cell reports 13(9): 1842-1854.

Narayanan R, Tuoc TC. 2014. Roles of chromatin remodeling BAF complex in neural differentiation and reprogramming. Cell and Tissue Research 356(3): 575-584.

Narlikar GJ, Sundaramoorthy R, Owen-Hughes T. 2013. Mechanisms and functions of ATP-dependent chromatin-remodeling enzymes. Cell 154(3): 490-503.

Neigeborn L, Carlson M. 1984. Genes Affecting the Regulation of Suc2 GeneExpression by Glucose Repression in Saccharomyces-Cerevisiae. Genetics 108(4): 845-858.

Nguyen H, Kerimoglu C, Pirouz M, Pham L, Kiszka KA, Sokpor G, Sakib MS, Rosenbusch J, Teichmann U, Seong RH, et al. 2018. Epigenetic Regulation by BAF Complexes Limits Neural Stem Cell Proliferation by Suppressing Wnt Signaling in Late Embryonic Development. Stem Cell Reports 10(6): 17341750.

Nguyen H, Sokpor G, Pham L, Rosenbusch J, Stoykova A, Staiger JF, Tuoc T. 2016. Epigenetic regulation by BAF (mSWI/SNF) chromatin remodeling complexes is indispensable for embryonic development. Cell Cycle 15(10): 1317-1324.

Nielsen JV, Thomassen M, Mollgard K, Noraberg J, Jensen NA. 2014. Zbtb20 Defines a Hippocampal Neuronal Identity Through Direct Repression of Genes That Control Projection Neuron Development in the Isocortex. Cerebral Cortex 24(5): 1216-1229.

Nieto M, Monuki ES, Tang H, Imitola J, Haubst N, Khoury SJ, Cunningham J, Gotz M, Walsh CA. 2004. Expression of Cux-1 and Cux-2 in the subventricular zone and upper layers II-IV of the cerebral cortex. Journal of Comparative Neurology 479(2): 168-180.

Ninkovic J, Steiner-Mezzadri A, Jawerka M, Akinci U, Masserdotti G, Petricca S, Fischer J, von Holst A, Beckers J, Lie CD, et al. 2013. The BAF complex interacts with Pax6 in adult neural progenitors to establish a neurogenic crossregulatory transcriptional network. Cell Stem Cell 13(4): 403-418.

Nitarska J, Smith JG, Sherlock WT, Hillege MMG, Nott A, Barshop WD, Vashisht AA, Wohlschlegel JA, Mitter R, Riccio A. 2016. A Functional Switch of NuRD Chromatin Remodeling Complex Subunits Regulates Mouse Cortical Development. Cell Reports 17(6): 1683-1698.

Noctor SC, Flint AC, Weissman TA, Dammerman RS, Kriegstein AR. 2001. Neurons derived from radial glial cells establish radial units in neocortex. Nature 409(6821): 714-720.

Noctor SC, Flint AC, Weissman TA, Wong WS, Clinton BK, Kriegstein AR. 2002. Dividing precursor cells of the embryonic cortical ventricular zone have morphological and molecular characteristics of radial glia. Journal of Neuroscience 22(8): 3161-3173.

Noctor SC, Martinez-Cerdeno V, Ivic L, Kriegstein AR. 2004. Cortical neurons arise in symmetric and asymmetric division zones and migrate through specific phases. Nature Neuroscience 7(2): 136-144.

Nott A, Nitarska J, Veenvliet JV, Schacke S, Derijck AAHA, Sirko P, Muchardt C, Pasterkamp RJ, Smidt MP, Riccio A. 2013. S-nitrosylation of HDAC2 
regulates the expression of the chromatin-remodeling factor Brm during radial neuron migration. Proceedings of the National Academy of Sciences of the United States of America 110(8): 3113-3118.

O'Leary DD, Chou SJ, Sahara S. 2007. Area patterning of the mammalian cortex. Neuron 56(2): 252-269.

O'Leary DDM, Sahara S. 2008. Genetic regulation of arealization of the neocortex. Current Opinion in Neurobiology 18(1): 90-100.

Ohkubo Y, Chiang C, Rubenstein JLR. 2002. Coordinate regulation and synergistic actions of BMP4, SHH and FGF8 in the rostral prosencephalon regulate morphogenesis of the telencephalic and optic vesicles. Neuroscience 111(1): 117.

Ohtaka-Maruyama C, Okamoto M, Endo K, Oshima M, Kaneko N, Yura K, Okado H, Miyata T, Maeda N. 2018. Synaptic transmission from subplate neurons controls radial migration of neocortical neurons. Science 360(6386): 313-317.

Parrish JZ, Kim MD, Jan LY, Jan YN. 2006. Genome-wide analyses identify transcription factors required for proper morphogenesis of Drosophila sensory neuron dendrites. Genes \& Development 20(7): 820-835.

Pedersen MT, Helin K. 2010. Histone demethylases in development and disease. Trends in cell biology 20(11): 662-671.

Pereira JD, Sansom SN, Smith J, Dobenecker MW, Tarakhovsky A, Livesey FJ. 2010. Ezh2, the histone methyltransferase of PRC2, regulates the balance between self-renewal and differentiation in the cerebral cortex. Proceedings of the National Academy of Sciences of the United States of America 107(36): 15957-15962.

Petrik D, Latchney SE, Masiulis I, Yun S, Zhang Z, Wu Jl, Eisch AJ. 2015. Chromatin Remodeling Factor Brg1 Supports the Early Maintenance and Late Responsiveness of Nestin-Lineage Adult Neural Stem and Progenitor Cells. Stem Cells 33(12): 3655-3665.

Phelan ML, Sif S, Narlikar GJ, Kingston RE. 1999. Reconstitution of a core chromatin remodeling complex from SWI/SNF subunits. Molecular Cell 3(2): 247-253.

Pinon MC, Tuoc TC, Ashery-Padan R, Molnar Z, Stoykova A. 2008. Altered molecular regionalization and normal thalamocortical connections in cortexspecific Pax6 knock-out mice. J Neurosci 28(35): 8724-8734.

Pontious A, Kowalczyk T, Englund C, Hevner RF. 2008. Role of intermediate progenitor cells in cerebral cortex development. Dev Neurosci 30(1-3): 24-32.

Poschl J, Grammel D, Dorostkar MM, Kretzschmar HA, Schuller U. 2013. Constitutive activation of beta-catenin in neural progenitors results in disrupted proliferation and migration of neurons within the central nervous system. Dev Biol 374(2): 319-332.

Pozniak CD, Pleasure SJ. 2006. A tale of two signals: Wnt and Hedgehog in dentate neurogenesis. Sci STKE 2006(319): pe5.

Puschendorf M, Terranova R, Boutsma E, Mao XH, Isono KI, Brykczynska U, Kolb C, Otte AP, Koseki H, Orkin SH, et al. 2008. PRC1 and Suv39h specify parental asymmetry at constitutive heterochromatin in early mouse embryos. Nature Genetics 40(4): 411-420.

Rakic P. 1972. Mode of cell migration to the superficial layers of fetal monkey neocortex. Journal of Comparative Neurology 145(1): 61-83. 
Rakic P, KnyiharCsillik E, Csillik B. 1996. Polarity of microtubule assemblies during neuronal cell migration. Proceedings of the National Academy of Sciences of the United States of America 93(17): 9218-9222.

Rallu M, Corbin JG, Fishell G. 2002. Parsing the prosencephalon. Nat Rev Neurosci 3(12): 943-951.

Rash BG, Grove EA. 2006. Area and layer patterning in the developing cerebral cortex. Curr Opin Neurobiol 16(1): 25-34.

Rash BG, Grove EA. 2007. Patterning the dorsal telencephalon: a role for sonic hedgehog? Journal of Neuroscience 27(43): 11595-11603.

Rice DS, Curran T. 2001. Role of the Reelin signaling pathway in central nervous system development. Annual Review of Neuroscience 24: 1005-1039.

Romero CDJ, Borrell V. 2017. Genetic maps and patterns of cerebral cortex folding. Current Opinion in Cell Biology 49: 31-37.

Ronan JL, Wu W, Crabtree GR. 2013. From neural development to cognition: unexpected roles for chromatin. Nature reviews. Genetics 14(5): 347-359.

Rowitch DH, Kriegstein AR. 2010. Developmental genetics of vertebrate glial-cell specification. Nature 468(7321): 214-222.

Roy A, Gonzalez-Gomez M, Pierani A, Meyer G, Tole S. 2014. Lhx2 Regulates the Development of the Forebrain Hem System. Cerebral Cortex 24(5): 1361-1372.

Sahara S, O'Leary DDM. 2009. Fgf10 Regulates Transition Period of Cortical Stem Cell Differentiation to Radial Glia Controlling Generation of Neurons and Basal Progenitors. Neuron 63(1): 48-62.

Saito T, Nakatsuji N. 2001. Efficient gene transfer into the embryonic mouse brain using in vivo electroporation. Developmental Biology 240(1): 237-246.

Sawa H, Kouike H, Okano H. 2000. Components of the SWI/SNF complex are required for asymmetric cell division in C-elegans. Molecular Cell 6(3): 617-624.

Saxena M, Agnihotri N, Sen J. 2018. Perturbation of canonical and non-canonical BMP signaling affects migration, polarity and dendritogenesis of mouse cortical neurons. Development 145(1).

Schaffer AE, Breuss MW, Caglayan AO, Al-Sanaa N, Al-Abdulwahed HY, Kaymakçalan H, Yılmaz C, Zaki MS, Rosti RO, Copeland B, et al. 2018. Biallelic loss of human CTNNA2, encoding aN-catenin, leads to ARP2/3 complex overactivity and disordered cortical neuronal migration. Nature genetics 50(8): 1093-1101.

Schmid MT, Weinandy F, Wilsch-Brauninger M, Huttner WB, Cappello S, Gotz M. 2014. The role of alpha-E-catenin in cerebral cortex development: radial glia specific effect on neuronal migration. Frontiers in Cellular Neuroscience 8: 215.

Sessa A, Mao CA, Hadjantonakis AK, Klein WH, Broccoli V. 2008. Tbr2 Directs Conversion of Radial Glia into Basal Precursors and Guides Neuronal Amplification by Indirect Neurogenesis in the Developing Neocortex. Neuron 60(1): 56-69.

Shen X, Liu Y, Hsu YJ, Fujiwara Y, Kim J, Mao X, Yuan GC, Orkin SH. 2008. EZH1 mediates methylation on histone $\mathrm{H} 3$ lysine 27 and complements $\mathrm{EZH} 2$ in maintaining stem cell identity and executing pluripotency. Molecular cell 32(4): 491-502.

Shetty AS, Godbole G, Maheshwari U, Padmanabhan H, Chaudhary R, Muralidharan B, Hou PS, Monuki ES, Kuo HC, Rema V, et al. 2013. Lhx2 regulates a cortex-specific mechanism for barrel formation. Proc Natl Acad Sci U S A 110(50): E4913-4921. 
Shi Y. 2007. Histone lysine demethylases: emerging roles in development, physiology and disease. Nature reviews. Genetics 8(11): 829-833.

Shibata M, Kurokawa D, Nakao H, Ohmura T, Aizawa S. 2008. MicroRNA-9 Modulates Cajal-Retzius Cell Differentiation by Suppressing Foxg1 Expression in Mouse Medial Pallium. Journal of Neuroscience 28(41): 10415-10421.

Shikanai M, Nakajima K, Kawauchi T. 2011. N-cadherin regulates radial glial fiberdependent migration of cortical locomoting neurons. Commun Integr Biol 4(3): 326-330.

Shimogori T, Banuchi V, Ng HY, Strauss JB, Grove EA. 2004. Embryonic signaling centers expressing BMP, WNT and FGF proteins interact to pattern the cerebral cortex. Development 131(22): 5639-5647.

Shimogori T, Ogawa M. 2008. Gene application with in utero electroporation in mouse embryonic brain. Development Growth \& Differentiation 50(6): 499-506.

Shinozaki K, Yoshida M, Nakamura M, Aizawa S, Suda Y. 2004. Emx1 and Emx2 cooperate in initial phase of archipallium development. Mechanisms of Development 121(5): 475-489.

Sild M, Ruthazer ES. 2011. Radial Glia: Progenitor, Pathway, and Partner. Neuroscientist 17(3): 288-302.

Silva CG, Peyre E, Nguyen L. 2019. Cell migration promotes dynamic cellular interactions to control cerebral cortex morphogenesis. Nat Rev Neurosci 20(6): 318-329.

Smith-Roe SL, Bultman SJ. 2013. Combined gene dosage requirement for SWI/SNF catalytic subunits during early mammalian development. Mamm Genome 24(12): 21-29.

Sokpor G, Castro-Hernandez R, Rosenbusch J, Staiger JF, Tuoc T. 2018. ATPDependent Chromatin Remodeling During Cortical Neurogenesis. Front Neurosci 12: 226.

Sokpor G, Xie Y, Rosenbusch J, Tuoc T. 2017. Chromatin Remodeling BAF (SWI/SNF) Complexes in Neural Development and Disorders. Front Mol Neurosci 10: 243.

Solecki DJ. 2012. Sticky situations: recent advances in control of cell adhesion during neuronal migration. Curr Opin Neurobiol 22(5): 791-798.

Son EY, Crabtree GR. 2014. The role of BAF (mSWI/SNF) complexes in mammalian neural development. Am J Med Genet C Semin Med Genet 166C(3): 333-349.

Staahl BT, Tang J, Wu W, Sun A, Gitler AD, Yoo AS, Crabtree GR. 2013. Kinetic analysis of npBAF to nBAF switching reveals exchange of SS18 with CREST and integration with neural developmental pathways. Journal of Neuroscience 33(25): 10348-10361.

Stiller JW, Yang C, Collen J, Kowalczyk N, Thompson BE. 2018. Evolution and expression of core SWI/SNF genes in red algae. J Phycol 54(6): 879-887.

Storm EE, Garel S, Borello U, Hebert JM, Martinez S, McConnell SK, Martin GR, Rubenstein JLR. 2006. Dose-dependent functions of Fgf8 in regulating telencephalic patterning centers. Development 133(9): 1831-1844.

Stoykova A, Treichel D, Hallonet M, Gruss P. 2000. Pax6 modulates the dorsoventral patterning of the mammalian telencephalon. J Neurosci 20(21): 8042-8050.

Strahl BD, Allis CD. 2000. The language of covalent histone modifications. Nature 403(6765): 41-45. 
Subramanian L, Remedios R, Shetty A, Tole S. 2009. Signals from the edges: The cortical hem and antihem in telencephalic development. Seminars in Cell \& Developmental Biology 20(6): 712-718.

Subramanian L, Tole S. 2009b. Mechanisms Underlying the Specification, Positional Regulation, and Function of the Cortical Hem. Cerebral Cortex 19: 190-I95.

Sugitani Y, Nakai S, Minowa O, Nishi M, Jishage K, Kawano H, Mori K, Ogawa M, Noda T. 2002. Brn-1 and Brn-2 share crucial roles in the production and positioning of mouse neocortical neurons. Genes Dev 16(14): 1760-1765.

Sur M, Rubenstein JL. 2005. Patterning and plasticity of the cerebral cortex. Science 310(5749): 805-810.

Tabata H, Nakajima K. 2001. Efficient in utero gene transfer system to the developing mouse brain using electroporation: Visualization of neuronal migration in the developing cortex. Neuroscience 103(4): 865-872.

Takawa M, Masuda K, Kunizaki M, Daigo Y, Takagi K, Iwai Y, Cho HS, Toyokawa G, Yamane Y, Maejima $K$, et al. 2011. Validation of the histone methyltransferase EZH2 as a therapeutic target for various types of human cancer and as a prognostic marker. Cancer science 102(7): 1298-1305.

Takiguchi-Hayashi K, Sekiguchi M, Ashigaki S, Takamatsu M, Hasegawa H, Suzuki-Migishima R, Yokoyama M, Nakanishi S, Tanabe Y. 2004. Generation of reelin-positive marginal zone cells from the caudomedial wall of telencephalic vesicles. Journal of Neuroscience 24(9): 2286-2295.

Tang LL, Nogales E, Ciferri C. 2010. Structure and function of SWI/SNF chromatin remodeling complexes and mechanistic implications for transcription. Progress in Biophysics \& Molecular Biology 102(2-3): 122-128.

Taverna E, Gotz M, Huttner AB. 2014. The Cell Biology of Neurogenesis: Toward an Understanding of the Development and Evolution of the Neocortex. Annual Review of Cell and Developmental Biology, Vol 30 30: 465-502.

Tea JS, Luo LQ. 2011. The chromatin remodeling factor Bap55 functions through the TIP60 complex to regulate olfactory projection neuron dendrite targeting. Neural Development 6.

Telley L, Agirman G, Prados J, Amberg N, Fievre S, Oberst P, Bartolini G, Vitali I, Cadilhac C, Hippenmeyer S, et al. 2019. Temporal patterning of apical progenitors and their daughter neurons in the developing neocortex. Science 364(6440): 547-+.

Teo JL, Ma H, Nguyen C, Lam C, Kahn M. 2005. Specific inhibition of CBP/betacatenin interaction rescues defects in neuronal differentiation caused by a presenilin-1 mutation. Proc Natl Acad Sci U S A 102(34): 12171-12176.

Tétreault N, Champagne M-P, Bernier G. 2009. The LIM homeobox transcription factor Lhx2 is required to specify the retina field and synergistically cooperates with Pax6 for Six6 trans-activation. Developmental Biology 327(2): 541-550.

Theil T, Alvarez-Bolado G, Walter A, Ruther U. 1999. Gli3 is required for Emx gene expression during dorsal telencephalon development. Development 126(16): 3561-3571.

Tole S, Ragsdale CW, Grove EA. 2000. Dorsoventral patterning of the telencephalon is disrupted in the mouse mutant extra-toes(J). Developmental Biology 217(2): 254-265.

Tolstorukov MY, Sansam CG, Lu P, Koellhoffer EC, Helming KC, Alver BH, Tillman EJ, Evans JA, Wilson BG, Park PJ, et al. 2013. Swi/Snf chromatin remodeling/tumor suppressor complex establishes nucleosome occupancy at 
target promoters. Proceedings of the National Academy of Sciences of the United States of America 110(25): 10165-10170.

Tonosaki M, Itoh K, Umekage M, Kishimoto T, Yaoi T, Lemmon VP, Fushiki S. 2014. L1cam Is Crucial for Cell Locomotion and Terminal Translocation of the Soma in Radial Migration during Murine Corticogenesis. Plos One 9(1).

Toresson H, Potter SS, Campbell K. 2000. Genetic control of dorsal-ventral identity in the telencephalon: opposing roles for Pax6 and Gsh2. Development 127(20): 4361-4371.

Torii M, Hashimoto-Torii K, Levitt P, Rakic P. 2009. Integration of neuronal clones in the radial cortical columns by EphA and ephrin-A signalling. Nature 461(7263): 524-528.

Toyoda R, Assimacopoulos S, Wilcoxon J, Taylor A, Feldman P, Suzuki-Hirano A, Shimogori T, Grove EA. 2010. FGF8 acts as a classic diffusible morphogen to pattern the neocortex. Development 137(20): 3439-3448.

Tuoc T, Dere E, Radyushkin K, Pham L, Nguyen H, Tonchev AB, Sun GQ, Ronnenberg A, Shi YH, Staiger JF, et al. 2017. Ablation of BAF170 in Developing and Postnatal Dentate Gyrus Affects Neural Stem Cell Proliferation, Differentiation, and Learning. Molecular Neurobiology 54(6): 4618-4635.

Tuoc TC, Boretius S, Sansom SN, Pitulescu ME, Frahm J, Livesey FJ, Stoykova A. 2013. Chromatin regulation by BAF170 controls cerebral cortical size and thickness. Dev Cell 25(3): 256-269.

Tuoc TC, Radyushkin K, Tonchev AB, Pinon MC, Ashery-Padan R, Molnar Z, Davidoff MS, Stoykova A. 2009. Selective cortical layering abnormalities and behavioral deficits in cortex-specific Pax6 knock-out mice. Journal of Neuroscience 29(26): 8335-8349.

Tuoc TC, Stoykova A. 2008. Trim11 modulates the function of neurogenic transcription factor Pax6 through ubiquitin-proteosome system. Genes \& Development 22(14): 1972-1986.

Urbanska M, Blazejczyk M, Jaworski J. 2008. Molecular basis of dendritic arborization. Acta Neurobiol Exp (Wars) 68(2): 264-288.

Valiente M, Ciceri G, Rico B, Marin O. 2011. Focal Adhesion Kinase Modulates Radial Glia-Dependent Neuronal Migration through Connexin-26. Journal of Neuroscience 31(32): 11678-11691.

Valiente M, Marin 0. 2010. Neuronal migration mechanisms in development and disease. Current Opinion in Neurobiology 20(1): 68-78.

Varambally S, Cao Q, Mani RS, Shankar S, Wang X, Ateeq B, Laxman B, Cao X, Jing X, Ramnarayanan K, et al. 2008. Genomic loss of microRNA-101 leads to overexpression of histone methyltransferase $\mathrm{EZH} 2$ in cancer. Science 322(5908): 1695-1699.

Vasileiou G, Ekici AB, Uebe S, Zweier C, Hoyer J, Engels H, Behrens J, Reis A, Hadjihannas MV. 2015. Chromatin-Remodeling-Factor ARID1B Represses Wnt/beta-Catenin Signaling. Am J Hum Genet 97(3): 445-456.

Viti J, Gulacsi A, Lillien L. 2003. Wnt regulation of progenitor maturation in the cortex depends on Shh or fibroblast growth factor 2. Journal of Neuroscience 23(13): 5919-5927.

Vyas A, Saha B, Lai E, Tole S. 2003. Paleocortex is specified in mice in which dorsal telencephalic patterning is severely disrupted. Journal of Comparative Neurology 466(4): 545-553. 
Wang W, Xue Y, Zhou S, Kuo A, Cairns BR, Crabtree GR. 1996. Diversity and specialization of mammalian SWI/SNF complexes. Genes \& development 10(17): 2117-2130.

Wang WD, Cote J, Xue Y, Zhou S, Khavari PA, Biggar SR, Muchardt C, Kalpana GV, Goff SP, Yaniv M, et al. 1996. Purification and biochemical heterogeneity of the mammalian SWI-SNF complex. Embo Journal 15(19): 5370-5382.

Wang XF, Lee RS, Alver BH, Haswel JR, Wang S, Mieczkowski J, Drier Y, Gillespie SM, Archer TC, Wu JN, et al. 2017. SMARCB1-mediated SWI/SNF complex function is essential for enhancer regulation. Nature genetics 49(2): 289-295.

Weinberg P, Flames N, Sawa H, Garriga G, Hobert O. 2013a. The SWI/SNF Chromatin Remodeling Complex Selectively Affects Multiple Aspects of Serotonergic Neuron Differentiation. Genetics 194(1): 189-+.

Weinberg P, Flames N, Sawa H, Garriga G, Hobert O. 2013b. The SWI/SNF chromatin remodeling complex selectively affects multiple aspects of serotonergic neuron differentiation. Genetics 194(1): 189-198.

Wen S, Li H, Liu J. 2009. Epigenetic background of neuronal fate determination. Prog Neurobio/ 87(2): 98-117.

Whitehouse I, Flaus A, Cairns BR, White MF, Workman JL, Owen-Hughes T. 1999. Nucleosome mobilization catalysed by the yeast SWI/SNF complex. Nature 400(6746): 784-787.

Whitford KL, Dijkhuizen P, Polleux F, Ghosh A. 2002. Molecular control of cortical dendrite development. Annu Rev Neurosci 25: 127-149.

Wiegreffe C, Simon R, Peschkes K, Kling C, Strehle M, Cheng J, Srivatsa S, Liu P, Jenkins NA, Copeland NG, et al. 2015. Bcl11a (Ctip1) Controls Migration of Cortical Projection Neurons through Regulation of Sema3c. Neuron 87(2): 311-325.

Wiese M, Walther N, Diederichs C, Schill F, Monecke S, Salinas G, Sturm D, Pfister SM, Dressel R, Johnsen SA, et al. 2017. The beta-catenin/CBPantagonist ICG-001 inhibits pediatric glioma tumorigenicity in a Wntindependent manner. Oncotarget 8(16): 27300-27313.

Wilsch-Brauninger M, Florio M, Huttner WB. 2016. Neocortex expansion in development and evolution - from cell biology to single genes. Curr Opin Neurobiol 39: 122-132.

Wilson SW, Rubenstein JL. 2000. Induction and dorsoventral patterning of the telencephalon. Neuron 28(3): 641-651.

Wolff E, Suplicki MM, Behr R. 2019. Primordial germ cells do not migrate along nerve fibres in marmoset monkey and mouse embryos. Reproduction 157(1): 101109.

Wolpert L. 1986. Citation-Classic - Positional Information and the Spatial Pattern of Cellular-Differentiation. Current Contents/Life Sciences(3): 19-19.

Wrobel CN, Mutch CA, Swaminathan S, Taketo MM, Chenn A. 2007. Persistent expression of stabilized beta-catenin delays maturation of radial glial cells into intermediate progenitors. Dev Bio/ 309(2): 285-297.

Wu H, Zhang Y. 2014. Reversing DNA methylation: mechanisms, genomics, and biological functions. Cell 156(1-2): 45-68.

Wu JI. 2012. Diverse functions of ATP-dependent chromatin remodeling complexes in development and cancer. Acta biochimica et biophysica Sinica 44(1): 54-69. 
Wu Jl, Lessard J, Olave IA, Qiu Z, Ghosh A, Graef IA, Crabtree GR. 2007. Regulation of dendritic development by neuron-specific chromatin remodeling complexes. Neuron 56(1): 94-108.

Xiang Y, Zhu Z, Han G, Lin H, Xu L, Chen CD. 2007. JMJD3 is a histone H3K27 demethylase. Cell Research 17(10): 850-857.

Xie Y, Castro-Hernández R, Sokpor G, Pham L, Narayanan R, Rosenbusch J, Staiger JF, Tuoc T. 2019. RBM15 Modulates the Function of Chromatin Remodeling Factor BAF155 Through RNA Methylation in Developing Cortex. Molecular Neurobiology.

Xie ZF, Ma XH, Ji WL, Zhou GD, Lu YZ, Xiang ZH, Wang YX, Zhang L, Hu YP, Ding $Y Q$, et al. 2010. Zbtb20 is essential for the specification of CA1 field identity in the developing hippocampus. Proceedings of the National Academy of Sciences of the United States of America 107(14): 6510-6515.

Xu C-R, Zaret KS. 2012. Chromatin "pre-pattern" and epigenetic modulation in the cell fate choice of liver over pancreas in the endoderm. Nucleus (Austin, Tex.) 3(2): 150-154.

Xu CD, Funahashi Y, Watanabe T, Takano T, Nakamuta S, Namba T, Kaibuchi K. 2015. Radial Glial Cell-Neuron Interaction Directs Axon Formation at the Opposite Side of the Neuron from the Contact Site. Journal of Neuroscience 35(43): 14517-14532.

Xu Q, Liu YY, Wang XM, Tan GH, Li HP, Hulbert SW, Li CY, Hu CC, Xiong ZQ, Xu $\mathrm{X}$, et al. 2018. Autism-associated CHD8 deficiency impairs axon development and migration of cortical neurons. Molecular Autism 9.

Xuan S, Baptista CA, Balas G, Tao W, Soares VC, Lai E. 1995. Winged helix transcription factor BF-1 is essential for the development of the cerebral hemispheres. Neuron 14(6): 1141-1152.

Xuan SH, Baptista CA, Balas G, Tao WF, Soares VC, Lai E. 1995. Winged Helix Transcription Factor Bf-1 Is Essential for the Development of the Cerebral Hemispheres. Neuron 14(6): 1141-1152.

Yan Z, Wang Z, Sharova L, Sharov AA, Ling C, Piao Y, Aiba K, Matoba R, Wang W, Ko MS. 2008. BAF250B-associated SWI/SNF chromatin-remodeling complex is required to maintain undifferentiated mouse embryonic stem cells. Stem Cells 26(5): 1155-1165.

Yao B, Christian KM, He C, Jin P, Ming GL, Song H. 2016. Epigenetic mechanisms in neurogenesis. Nat Rev Neurosci 17(9): 537-549.

Yoon KJ, Vissers C, Ming GL, Song HJ. 2018. Epigenetics and epitranscriptomics in temporal patterning of cortical neural progenitor competence. Journal of Cell Biology 217(6): 1901-1914.

Yoshida M, Assimacopoulos S, Jones KR, Grove EA. 2006. Massive loss of CajalRetzius cells does not disrupt neocortical layer order. Development 133(3): 537545.

Yu Y, Chen Y, Kim B, Wang H, Zhao C, He X, Liu L, Liu W, Wu LM, Mao M, et al. 2013. Olig2 targets chromatin remodelers to enhancers to initiate oligodendrocyte differentiation. Cell 152(1-2): 248-261.

Yun K, Potter S, Rubenstein JL. 2001. Gsh2 and Pax6 play complementary roles in dorsoventral patterning of the mammalian telencephalon. Development 128(2): 193-205.

Zembrzycki A, Griesel G, Stoykova A, Mansouri A. 2007. Genetic interplay between the transcription factors Sp8 and Emx2 in the patterning of the forebrain. Neural Development 2. 
Zhan X, Shi X, Zhang Z, Chen Y, Wu JI. 2011. Dual role of Brg chromatin remodeling factor in Sonic hedgehog signaling during neural development. Proc Natl Acad Sci U S A 108(31): 12758-12763.

Zhang T, Zhang S, Song X, Zhao X, Hou C, Li Z, Gao J. 2019. Loss of Lgl1 Disrupts the Radial Glial Fiber-guided Cortical Neuronal Migration and Causes Subcortical Band Heterotopia in Mice. Neuroscience 400: 132-145.

Zhao LN, Li J, Ma YL, Wang JT, Pan W, Gao K, Zhang ZR, Lu TL, Ruan YY, Yue WH, et al. 2015. Ezh2 is involved in radial neuronal migration through regulating Reelin expression in cerebral cortex. Scientific Reports 5.

Zhou CJ, Zhao CJ, Pleasure SJ. 2004. Wnt signaling mutants have decreased dentate granule cell production and radial glial scaffolding abnormalities. Journal of Neuroscience 24(1): 121-126.

Zhuo L, Theis M, Alvarez-Maya I, Brenner M, Willecke K, Messing A. 2001. hGFAPcre transgenic mice for manipulation of glial and neuronal function in vivo. Genesis 31(2): 85-94.

Ziller MJ, Edri R, Yaffe Y, Donaghey J, Pop R, Mallard W, Issner R, Gifford CA, Goren A, Xing J, et al. 2015. Dissecting neural differentiation regulatory networks through epigenetic footprinting. Nature 518(7539): 355-359.

\section{List of figures}

Figure 1.1 Schematics showing general patterning of the mouse telencephalon or

cortex: 3

Figure 1.2 Synopsis of regulatory events during cortical patterning: 4

Figure 1.3 Illustration showing the generation and placement of excitatory neurons during cortical formation:

Figure 1.4 Schema showing modes of radial neuronal migration in the cortex:

Figure 1.5 Model showing the known forms of BAF complexes in neural cells and genetic scheme for BAF complex knockout: 20

Figure 1.6 Schema showing overview of the multiple neurodevelopmental functions of the BAF (mSWI/SNF) complex: 21

Figure 2.1 The expressions of BAF155 and BAF170 are indispensable for embryonic development: .35 
Figure 2.2 Expression of BAF subunits in telencephalon-specific dcKO_FoxG1-Cre mutants: .38

Figure 2.3 Expression of BAF subunits in embryos of TAM-inducible full dcKO_CAGCre mutants:. .39

Figure 2.4 BAF complexes control the level of $\mathrm{H} 3 \mathrm{~K} 27 \mathrm{me} 3$ in the brain and whole embryo during development: 42

Figure 3.1 The cortical hem phenotype of the early developing BAF complex mutant forebrain resembles that of Lhx2 null mutant: .53

Figure 3.2 BAF complex cooperates with LHX2 to direct cortical development: .57

Figure 3.3 Increased expression of cortical hem-specific markers in dcKO_Emx1-Cre pallium: .63

Figure 3.4 Early loss of BAF complex in the developing dTel distorts cortical patterning: 66

Figure 3.5 The BAF complex-ablated hem is incapable of hippocampal specification and neocortical patterning: 69

Figure 3.6 BAF complex acts independent of WNT signaling to suppress hem expansion: .71

Figure 3.7 The morphogenetic function of BAF complex is evolutionarily refined:....74 Figure 3.8 Graphical illustration of normal and BAF155/170 knockout-induced dTel abnormal patterning: .74

Figure S3.1 Emx1-Cre-mediated deletion of BAF155 and BAF170 causes early loss of BAF complex in the dTel: .85

Figure S3.2 Expansion of the cortical hem occurs early in corticogenesis following ablation of BAF complex: .86 
Figure S3.3 Single or late double knockout of BAF155/BAF170 does not cause expansion of the cortical hem:

Figure S3.4 Reduced mitotic exit of hem progenitors and increased apoptosis of Reelin+ hem progenies due to loss of BAF complex in dTel: 88

Figure S3.5 The dorsal telencephalon is not properly patterned when BAF complex is deleted in developing mouse cortex: .89

Figure S3.6 Co-expression and/or interaction of BAF complex and LHX2 in the developing cortex: .90

Figure 4.1 BAF complex inactivation causes massive cortical malformation partly due to defective neuronal migration in the early developing cortex: 105

Figure 4.2 Cortical layer formation is improper in the absence of BAF complex due to loss of cell adhesion and glial fiber scaffolds: 109

Figure 4.3 Neuron-specific deletion of BAF complex alters neuronal differentiation programs leading to defective neuronal migration:

Figure 4.4 Migrating neurons without BAF complex exhibit abnormal polarity and radial mis-guidance:

Figure 4.5 BAF complex regulates multipolar-to-bipolar neuronal morphology transition during radial migration: 120

Figure 4.6 BAF complex modulates WNT signaling to drive cortical lamination: ....123 Figure 4.7 Schematic synopsis of how BAF complex mainly influences radial neuronal migration: 124

Figure S4.1 Scheme for dcKO generation: 138

Figure S4.2: Loss of BAFs in the dcKO_Nex-Cre cortex 139 
Figure S4.3 Loss of neuronal BAF complex causes cortical hypoplasia and corpus callosum defects: 140

\section{List of abbreviations}

$\begin{array}{ll}\text { A1 } & \text { Primary auditory cortex } \\ \text { AP } & \text { Apical progenitor } \\ \text { ATP } & \text { Adenosine triphosphate } \\ \text { BAF } & \text { Brg1/Brm associated factor } \\ \text { BM } & \text { Bipolar neuron } \\ \text { BMP } & \text { Bone morphogenetic protein } \\ \text { BP } & \text { Basal progenitor } \\ \text { BRG1 } & \text { BRM/SWI2 related gene 1 } \\ \text { BRM } & \text { Brahma } \\ \text { CP } & \text { Cortical plate } \\ \text { Ctip2 } & \text { Chicken ovalbumin upstream promoter transcription factor- } \\ & \text { interacting proteins 2 } \\ \text { Cux1 } & \text { Cut Like Homeobox 1 } \\ \text { Cx } & \text { Cortex } \\ \text { DAPI } & \text { 4,6-dasmindino-2-phenylindol } \\ \text { dcKO } & \text { Double conditional knockout } \\ \text { dTel } & \text { Dorsal telencephalon } \\ \text { E } & \text { Embryonic day } \\ \text { eGFP } & \text { Enhanced green fluorescent protein } \\ \text { Emx1 } & \end{array}$




\begin{tabular}{|c|c|}
\hline $\mathrm{F}$ & Frontal cortex \\
\hline $\mathrm{FI}$ & Floxed \\
\hline GFP & Green fluorescent protein \\
\hline GLAST & Glutamate aspartate transporter \\
\hline hGFAP & Human glial fibrillary acidic protein \\
\hline $\mathrm{IHC}$ & Immunohistochemistry \\
\hline $\mathrm{ISH}$ & In situ hybridization \\
\hline IUE & In utero electroporation \\
\hline IZ & Intermediate zone \\
\hline IZ & Intermediate zone \\
\hline MN & Multipolar neuron \\
\hline$M Z$ & Marginal zone \\
\hline$n B A F$ & Neuronal BAF \\
\hline npBAF & Neural progenitor BAF \\
\hline$P$ & Postnatal day \\
\hline Pax6 & Paired box 6 \\
\hline PRC2 & Polycomb repressor complex 2 \\
\hline RGs & Radial glial cells \\
\hline RNA-seq & Ribonucleic acid sequencing \\
\hline S1 & Primary sensory cortex \\
\hline SEM & Standard error of the mean \\
\hline Sox2 & SRY-related HMG transcription factor 2 \\
\hline SVZ & Subventricular zone \\
\hline SWI/SNF & SWItch/Sucrose Non-Fermentable \\
\hline
\end{tabular}




$\begin{array}{ll}\text { TAM } & \text { Tamoxifen } \\ \text { Tbr1 } & \text { T-box brain 1 } \\ \text { Tbr2 } & \text { T-box brain 2 } \\ \text { TF } & \text { Transcription factor } \\ \text { UP } & \text { Unipolar neuron } \\ \text { V1 } & \text { Primary visual cortex } \\ \text { VTel } & \text { Ventral telencephalon } \\ \text { VZ } & \text { Ventricular zone } \\ \text { WB } & \text { Western blot } \\ \text { WNT } & \text { Wingless/Int } \\ \text { WNTi } & \text { Wingless/Int inhibitor } \\ \text { WT } & \text { Wild-type }\end{array}$

\section{Acknowledgements}

I wish to thank members of my thesis advisory committee for stirring the affairs of my studies to a decent completion. Special thanks to Prof. Dr. Jochen Staiger for allowing me to be a doctoral student in the institute of Neuroanatomy. The effect of his scholarly acumen and coaching profundity during my studentship cannot go unappreciated.

Dr. Tran Tuoc, the architect of my doctoral training/journey and overseer of my day-today scientific activities and growth, is utterly appreciated. I am very grateful to him for especially introducing me to the BAF complex and brain development research fraternity. Mrs. Linh Pham, our indefatigable technical assistant, is similarly acknowledged for her great support during my studies. 
I would like to thank current and past members of the Institute of Neuroanatomy in the University Medical Center Goettingen for various forms of support in the course of my studies. Particularly, Drs. Huong Nguyen, Joachim Rosenbusch and Yuanbin Xie, are greatly appreciated for productive scientific discussions and collaborations. All other people who collaborated in carrying out the various studies are also appreciated, especially Prof. Rüdiger Behr, Prof. Anastassia Stoykova, and Prof. Shubha Tole.

Many thanks to the following funding agencies for their financial support: the DAAD and Ministry of Education (MoE), Ghana for PhD scholarship and monthly stipends award, the Goettingen Graduate Center for Neurosciences, Biophysics, and Molecular Biosciences (GGNB) for doctoral training finishing grant, and the German research foundation (DFG) and Schram-Stiftung for bulk research grants.

Final thanks goes to my family. My parents, siblings, endearing children (Karen and Phoebe), and breathtakingly supportive wife (Gifty), are extremely appreciated for their tender love, care, prayers, and for sticking around through thick and thin. 